\author{
Universidade de SÃo Paulo \\ Instituto de Física de SÃo Carlos \\ Departamento de Física e Informática
}

\title{
Magnetotransporte e ferromagnetismo Hall em heteroestruturas semicondutoras magnéticas
}

\author{
Henrique Jota de Paula Freire
}

Tese apresentada ao Instituto de Física de São Carlos, Universidade de São Paulo, para obtenção do Título de Doutor em Ciências: Física Básica

Orientador: Prof. Dr. José Carlos Egues

SÃo CARlOS

2004 
Freire, Henrique Jota de Paula

Magnetotransporte e ferromagnetismo Hall em heteroestruturas semicondutoras magnéticas / Henrique Jota de Paula Freire. - São Carlos, 2004.

190 p.

Tese (Doutorado) - Universidade de São Paulo. Instituto de Física de São Carlos. Programa de Pós-graduação em Física.

Orientador: Prof. Dr. José Carlos Egues.

1. Semicondutor magnético. 2. Estrutura eletrônica. 3. Magnetotransporte quântico. 4. Ferromagnetismo Hall. 5. Poço quântico. I. Título.

Este texto foi preparado em $\mathrm{LT}_{\mathrm{E} X}$. 


Dedico esta tese à minha esposa Maria Alice e à minha filha Flávia. 



\section{Agradecimentos}

- Ao Prof. Egues, pela oportunidade de realizar este trabalho, pelo entusiasmo científico e pelas discussões.

- Ao Prof. Klaus, pela amizade e pela colaboração fundamental em várias etapas desta tese e em outros projetos.

- Aos meus queridos pais Tarcísio e Elizabeth, sempre presentes, apoiando e aconselhando. E às minhas queridas irmãs Tatiana e Gabriela, pelo carinho, paciência e impaciência. Saudades de vocês.

- Às famílias Rugai e Vitta, que me receberam com tanto carinho.

- Aos colegas de sala Fabrício e Fernando, companheiros de vários anos.

- À Cristina, secretária do grupo de teoria, pela amizade, companhia, paciência e ajuda.

- À Wladerez, secretária da pós-graduação, sempre prestativa e simpática.

- Ao Paulo Alexandre, amigo fiel e sempre presente.

- À todos os meus queridos amigos, que têm me acompanhado e incentivado durante todos estes anos. Nos divertimos, rimos e choramos, vivemos.

- Aos demais colegas do Instituto de Física de São Carlos, estudantes, funcionários e professores, pela companhia de tantos anos.

- Às funcionárias da biblioteca pela presteza e gentileza no atendimento.

- Para concluir, eu gostaria de agradecer à todos que direta ou indiretamente contribuíram para que eu me sentisse tão bem nesta minha estadia em São Carlos.

Este trabalho foi financiado pela Fundação de Amparo à Pesquisa do Estado de São Paulo (FAPESP-99/06868-3). 



\section{Resumo}

Heteroestruturas digitais magnéticas (DMHs) são estruturas semicondutoras em que a distribuição de impurezas magnéticas $(\mathrm{Mn})$ restringe-se a alguns arranjos bidimensionais (monocamadas) regularmente espaçados entre si. Na presença de um campo magnético, a interação de troca sp-d entre os momentos magnéticos localizados e os portadores itinerantes é responsável por um desdobramento de spin gigante, da ordem ou até superior que a separação cíclotron dos níveis de Landau. Aqui eu calculo a estrutura eletrônica de poços quânticos digitais magnéticos do grupo II-VI. Resolvo as equações de Kohn-Sham da teoria do funcional da densidade dependente de spin na aproximação de massa efetiva. Eu então calculo diversas propriedades magnetoópticas e de transporte relevantes experimentalmente. Em particular, eu investigo a física dependente de spin presente nestes sistemas sob dois diferentes pontos de vista. Primeiramente o enfoque é no efeito do magnetismo do Mn sobre o potencial dependente de spin da interação de troca $s p-d$, em particular nos efeitos da aglomeração antiferromagnética e da diluição do seu perfil de concentração (segregação e interdifusão). Ao considerar estes efeitos eu reproduzo resultados experimentais para desdobramento de spin $\Delta E$ e tempos de espalhamento de spin $\tau_{s f}$ [S. A. Crooker et al., Phys. Rev. Lett. 75, 505 (1995); Phys. Rev. B 61, 1736 (2000)]. Na segunda parte eu mudo o enfoque para a física de gases de elétrons bidimensionais (2DEGs) altamente polarizados e mostro a importância da forte dependência de spin das contribuições de muitos corpos (troca e correlação) presentes nestes sistemas. Em particular, estes efeitos são relevantes para o surgimento de fases de ferromagnetismo de efeito Hall quântico. Eu calculo o magnetotransporte no regime de efeito Hall quântico para DMHs baseadas em ZnSe e CdTe. Meus resultados reproduzem resultados experimentais [R. Knobel et al., Phys. Rev. B 65, 235327 (2002); J. Jaroszyński et al., Phys. Rev. Lett. 89, 266802 (2002)] para a dependência com o campo magnético, com a temperatura, o aparecimento de picos anômalos e o surgimento de curvas de histerese em várias propriedades físicas. 



\section{Abstract}

Digital magnetic heterostructures (DMHs) are semiconductor structures with magnetic impurities $(\mathrm{Mn})$ restricted to some planar arrangements (monolayers) regularly spaced. In the presence of an external magnetic field, the sp-d exchange interaction between the localized magnetic moments and the itinerant carriers is responsible for a giant spin splitting, of the order of, or even greater than, the cyclotron separation between Landau levels. Here I calculate the electronic structure of group II-VI digital magnetic quantum wells. I solve the Kohn-Sham equations of the spin-density functional theory within the effective mass approximation. Then I calculate some magneto-optical and transport properties which are experimentally relevant. In particular, I investigate the spin dependent physics of these systems from two different points of view. First, I focus on effects of the Mn magnetism on the sp-d exchange spin dependent potential, particularly the effect of antiferromagnetic clustering and the effect of dilution (segregation and interdiffusion) of the Mn content profile. By considering these effects I reproduce experimental results for the spin splitting $\Delta E$ and spin scattering times $\tau_{s f}$ [S. A. Crooker et al., Phys. Rev. Lett. 75, 505 (1995); Phys. Rev. B 61, 1736 (2000)]. In the second part I move on to the physics of spin-polarized two-dimensional electron gases (2DEGs), and show the relevance of the strong dependence of the many-body contributions (exchange and correlation) with the spin polarization. In particular, these effects are relevant for the development of quantum Hall ferromagnetic phases. I calculate magnetotransport in the quantum Hall effect regime for DMHs consisting of ZnSe and CdTe. My results reproduce experimental results [R. Knobel et al., Phys. Rev. B 65, 235327 (2002); J. Jaroszyński et al., Phys. Rev. Lett. 89, 266802 (2002)] for the dependence with magnetic field, temperature, development of anomalous resistivities spikes and hysteretic behaviors in many physical properties. 



\section{Sumário}

Resumo ix

Abstract $\quad$ xi

Lista de Figuras $\quad$ xiv

$\begin{array}{ll}\text { Lista de Tabelas } & \text { xvii }\end{array}$

Lista de Símbolos $\quad$ xxi

1 Introdução 1

1.1 Sistema "modelo" e resumo dos resultados . . . . . . . . . . . . 3

1.2 Abordagem teórica . . . . . . . . . . . . . . . 4

1.3 Organização da tese . . . . . . . . . . . . . . . . 4

1.4 Heteroestruturas digitais magnéticas . . . . . . . . . . . 5

I Efeitos de Aglomeração e Diluição do Mn sobre as Propriedades Magnetoópticas em DMHs 17

2 Aglomeração Antiferromagnética e Diluição $\quad 19$

2.1 Introdução . . . . . . . . . . . . . . . . . . . . 20

2.2 Aglomeração antiferromagnética e concentração efetiva . . . . . . 22

2.3 Diluição: segregação e interdifusão . . . . . . . . . . . . . 27

2.4 Perfis modelo . . . . . . . . . . . . . . . . . . . . . . . . . 32

2.5 Perfis de concentração: nominal e efetiva . . . . . . . . . . . . 35

2.6 Discussões . . . . . . . . . . . . . . . . . . . . . . . . . . . . 42

3 Desdobramento de Spin e Tempo de Espalhamento 45

3.1 Introdução . . . . . . . . . . . . . . . . . . . . . 46

3.2 Sistema modelo e abordagem teórica . . . . . . . . . . . . . . . 48

3.3 Resultados: desdobramento de spin . . . . . . . . . . . . . . . 51

3.4 Resultados: tempo de espalhamento de spin . . . . . . . . . . . 59 
3.5 Discussões . . . . . . . . . . . . . . . . . . . . . 67

II Magnetotransporte e Ferromagnetismo Itinerante 71

4 Gás de Elétrons Bidimensional e Quantização de Landau $\quad 73$

4.1 Introdução . . . . . . . . . . . . . . . . . . . . . . 74

4.2 Estrutura eletrônica: DFT/LSDA . . . . . . . . . . . . . 77

4.3 Campo magnético e quantização de Landau . . . . . . . . . . . . 90

4.4 Magnetotransporte no regime quântico . . . . . . . . . . . . . . . 99

4.5 Discussões e resumo da formulação . . . . . . . . . . . . . . . 106

5 Dependência com a Temperatura das Oscilações de SdH 109

5.1 Introdução . . . . . . . . . . . . . . . . . . . . . . . . . . 110

5.2 Resumo dos resultados experimentais . . . . . . . . . . . . . . . 111

5.3 Resultados para magnetotransporte e energia total . . . . . . . . 120

5.4 Discussões . . . . . . . . . . . . . . . . . . . . . . . . 130

6 Ferromagnetismo Itinerante no Regime de Efeito Hall Quântico133

6.1 Introdução . . . . . . . . . . . . . . . . . . . . . 135

6.2 Resumo dos resultados experimentais . . . . . . . . . . . . . 136

6.3 Resultados teóricos . . . . . . . . . . . . . . . . . . . . . 145

6.4 Discussões . . . . . . . . . . . . . . . . . . . . . . . . . 159

$\begin{array}{llr}7 & \text { Conclusões } & 163\end{array}$

$\begin{array}{ll}\text { Apêndices } & 168\end{array}$

A Tempo de espalhamento de spin em DMHs $\quad 169$

A.1 Introdução . . . . . . . . . . . . . . . . . . . . . . . . . 169

A.2 Elemento de matriz do espalhamento de spin . . . . . . . . . . . . 170

A.3 Média sobre as impurezas . . . . . . . . . . . . . . . . . . 172

A.4 Taxa de espalhamento de spin . . . . . . . . . . . . . . . 173

$\begin{array}{lr}\text { Referências Bibliográficas } & 175\end{array}$ 


\section{Lista de Figuras}

1.1 Heteroestrutura digital magnética . . . . . . . . . . . . 6

1.2 Estrutura de bandas sem e com campo magnético. . . . . . . . . 7

1.3 Estrutura de banda ao redor do ponto $\Gamma \ldots \ldots$. . . . . . . 8

1.4 Perfil de potencial, sub-bandas e funções de onda de uma DMH. . 15

2.1 Spin efetivo de semicondutores semimagnéticos. . . . . . . . . . 23

2.2 Tipos de aglomerados magnéticos. . . . . . . . . . . . . . . . . . . 24

2.3 Estrutura cúbica do semicondutor semimagnético (Zn,Mn)Se. . . . 24

2.4 Concentrações efetivas para vários modelos de aglomerados. . . . 26

2.5 Modelo termodinâmico para segregação. . . . . . . . . . . . . 28

2.6 Perfis de interdifusão de fontes planar, semi-infinita e barreira . . 31

2.7 Perfis de concentração nominal: ideal, segregação e interdifusão. . 34

2.8 Probabilidades de aglomeração num sistema diluído. . . . . . . . . 36

2.9 Perfis de concentração efetivos $\bar{x}(z) \ldots \ldots$. . . . . . . . . 37

2.10 Comparação dos modelos de aglomeração considerando diluição. 38

2.11 Concentração média $\langle\bar{x}\rangle \ldots$. . . . . . . . . . . . . . . . . . . 39

2.12 Curvas de nível da concentração efetiva média. . . . . . . . . . . . 40

2.13 Curvas de nível da concentração efetiva $x_{\text {eff }} \ldots \ldots$. . . . . . . 41

2.14 Esquema dos efeitos de aglomeração e diluição. . . . . . . . . . . . 43

3.1 Poço quântico digital magnético ideal, desdobramento de spin e transições opticamente ativas. . . . . . . . . . . . . . . 46

3.2 Desdobramento de spin gigante experimental a $T=4.2 \mathrm{~K} . \quad \ldots \quad . \quad 47$

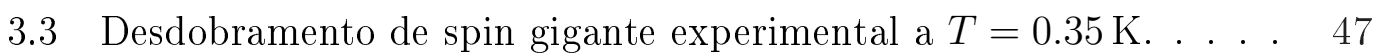

3.4 Potencial efetivo e funções de onda de elétrons e buracos-pesados. 50

3.5 Desdobramento de spin usando concentração nominal. . . . . . . . 53

3.6 Desdobramento de spin usando concentração efetiva . . . . . . . . 54

3.7 Resultados teóricos para o desdobramento de spin a $T=0.35 \mathrm{~K} . \quad .55$

3.8 Resultados teóricos para o desdobramento de spin a $T=4.2 \mathrm{~K}$. . 55

3.9 Desdobramento de spin $\Delta E^{-}$em função de $N_{b} \ldots \ldots$. . . . 57

3.10 Tempo de espalhamento de spin experimental. . . . . . . . . . . 59 
3.11 Ilustração do mecanismo de espalhamento de spin. . . . . . . . . . 59

3.12 Fator de sobreposição $\Gamma\left(N_{b}, \ell\right)$ em função de $N_{b}$ e $\ell$. . . . . . . 62

3.13 Tempo de espalhamento de spin médio usando concentração nominal. 64

3.14 Tempo de espalhamento de spin médio usando concentração efetiva. 65

3.15 Tempo de espalhamento de spin em função de $N_{b}$. . . . . . . . 67

4.1 Poço quântico digital magnético dopado por modulação . . . . . . 74

4.2 Ingredientes do potencial autoconsistente de uma DMH. . . . . . 76

4.3 Fator de escala de spin em várias parametrizações de $v_{x c}(z)$. . . 84

4.4 Potencial de troca-correlação para GaAs e ZnSe. . . . . . . . . . . 89

4.5 Densidade de estados bidimensional, sem e com desordem. . . . . 92

4.6 Níveis de landau, energia orbital e magnetização não-interagentes. 98

4.7 Geometria Hall . . . . . . . . . . . . . . . . . . . . . . . . . 100

4.8 Explicação para os platôs do efeito Hall em termos de estados localizados. . . . . . . . . . . . . . . . . . . 102

4.9 Densidade de estados total e estendida . . . . . . . . . . . . . . 104

4.10 Resistividades longitudinal $\rho_{x x}$ e transversal $\rho_{x y}$ de um 2DEG . . 105

5.1 Resistividade Hall experimental . . . . . . . . . . . . . . . . . 113

5.2 Oscilações de Shubnikov-de Haas experimental . . . . . . . . . . . 114

5.3 Magnetização experimental . . . . . . . . . . . . . . . . . . . . . 115

5.4 Oscilações de Shubnikov-de Haas experimental em função de 1/B. 116

5.5 Diagrama de níveis de Landau do modelo do grupo experimental . 117

5.6 Perfil de potencial do poço quântico de $n$-(Zn,Cd,Mn)Se. . . . . . 122

5.7 Diagrama dos níveis de Landau e de densidade bidimensional. . . 123

5.8 Magnetotransporte em poços de $n$-(Zn,Cd,Mn)Se . . . . . . . . 125

5.9 Resultados na aproximação Hartree. . . . . . . . . . . . . . . . 127

5.10 Energia total do 2DEG em função do campo magnético . . . . . . 129

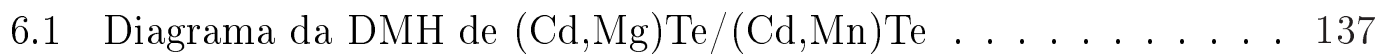

6.2 Resultados experimentais para magnetotransporte . . . . . . . . . 139

6.3 Resultados experimentais de $\rho_{x x}$ em função de $\theta$. . . . . . . . . 140

6.4 Diagrama de níveis de Landau do grupo experimental . . . . . . . 141

6.5 Resultados experimentais demonstrando histerese . . . . . . . . . 142

6.6 Perfil de potencial do poço quântico de (Cd,Mg)Te/(Cd,Mn)Te . . 146

6.7 Diagrama dos níveis de Landau e densidade do 2DEG . . . . . . . 147

6.8 Resultados teóricos para os efeitos SdH e IQHE . . . . . . . . . . 149

6.9 Ampliação da Fig. 6.8(a) na região do pico anômalo . . . . . . . . 150

6.10 Simulação do pico anômalo em função da temperatura . . . . . 151

6.11 Simulação do pico anômalo em função da ângulo do campo magnético152 
6.12 Dependência do sentido de $B_{c}$ com o sinal de $g^{*} \ldots$. . . . . . 155

6.13 Análise do ciclo de histerese em função da temperatura. . . . . . . 156

6.14 Energia total em função do campo magnético . . . . . . . . . . . 157

6.15 Primeira derivada total da energia total com relação ao campo magnético . . . . . . . . . . . . . . . . . . . . 158 


\section{Lista de Tabelas}

2.1 Probabilidade de um íon de Mn pertencer a um aglomerado. . . . 25

3.1 Parâmetros da simulação do poço quântico digital magnético. . . 49

3.2 Comprimentos característicos que melhor ajustam os dados experimentais para $\Delta E \times B \ldots \ldots \ldots \ldots$

4.1 Nomenclatura utilizada na Fig. 4.3. . . . . . . . . . . . 85

5.1 Características do DMQW de (Zn,Cd,Mn)Se . . . . . . . . . . . 112

5.2 Parâmetros da simulação do DMQW de (Zn,Cd,Mn)Se . . . . . 121

6.1 Características do DMQW de (Cd,Mn)Te . . . . . . . . . . 138

6.2 Parâmetros da simulação do DMQW de (Cd,Mn)Te . . . . . . . 145 


\section{Lista de Símbolos}

$d \quad$ Largura de uma (mono)camada. Usado nas funções $\wp(z)$.

$\Delta E_{\text {sat }}$ Energia de saturação do desdobramento de spin induzido pela interação de troca $s p-d$.

$\Delta E^{ \pm} \quad$ Desdobramento de spin total com polarização $\sigma= \pm 1$.

$E_{e, h h}^{ \pm 1} \quad$ Energia da transição elétron-buraco-pesado com polarização $\sigma= \pm 1$.

$E_{e, h h}^{0} \quad$ Energia da transição elétron-buraco-pesado a campo nulo.

$H_{n} \quad$ Polinômio de Hermite de ordem $n$.

L L Largura do poço quântico.

$L_{B} \quad$ Largura da barreira lateral.

$L_{D} \quad$ Largura de depleção.

$L_{N} \quad$ Largura da região $n$-dopada.

$L_{S} \quad$ Largura do espaçador.

$\ell \quad$ Comprimento característico (segregação ou interdifusão) do perfil de concentração $\wp(z)$.

$\ell_{0} \quad$ Comprimento magnético.

$n \quad$ Densidade de elétrons por unidade de volume.

$n_{e} \quad$ Densidade de elétrons na região dopada.

$n_{i} \quad$ Densidade de impurezas doadoras.

$n_{s}, n_{2 D}$ Densidade bidimensional de partículas, por unidade de área.

$N_{b} \quad$ Número de barreiras magnéticas.

$P_{i} \quad$ Probabilidade de um íon de Mn participar de um aglomerado de $i$ íons.

$\wp(z) \quad$ Função perfil. Pode ser quadrada (sqr), exponencial (exp) e função-erro (erf).

$r_{s} \quad$ Raio de Wigner-Seitz, usado em unidades de raio de Bohr efetivo.

$S \quad$ Spin total do Mn. $S=5 / 2$.

$\bar{S} \quad$ Spin efetivo do Mn. 
$S_{i} \quad$ Spin médio de um aglomerado com $i$ íons de Mn.

$S_{z} \quad$ Componente $z$ do spin do Mn.

T Temperatura.

$T_{0} \quad$ Temperatura efetiva do Mn.

$T_{e} \quad$ Temperatura eletrônica de portadores não termalizados.

$T_{L} \quad$ Temperatura da rede cristalina.

$v_{0} \quad$ Offset estrutural do poço quântico.

$v_{0}(z) \quad$ Perfil de potencial estrutural do poço quântico.

$v_{b} \quad$ Offset estrutural das barreiras magnéticas.

$v_{b}(z) \quad$ Perfil de potencial estrutural das barreiras magnéticas.

$v_{h} \quad$ Potencial de Hartree.

$v_{s p-d} \quad$ Contribuição da interação de troca $s p-d$ para o potencial.

$v_{s p-d}(z)$ Perfil de potencial de troca $s p-d$.

$v_{x c} \quad$ Potencial de troca e correlação.

$x \quad$ Concentração molar nominal de Mn.

$\bar{x} \quad$ Concentração molar efetiva de $\mathrm{Mn}$.

$x_{N N} \quad$ Concentração molar média de Mn considerando três monocamadas consecutivas.

$x_{p} \quad$ Concentração molar planar (nominal) de Mn em uma camada ou monocamada.

$\langle\bar{x}\rangle \quad$ Concentração efetiva média.

$x_{\text {eff }} \quad$ Concentração efetiva que considera a superposição do perfil $\bar{x}(z)$ com as funções de onda dos portadores.

$Z_{j} \quad$ Coordenada da $j$-ésima (mono)camada. Usado em $\wp(z)$.

$\Lambda \quad$ Superescrito que indica banda de condução $(\Lambda=e)$ ou banda de valência $(\Lambda=h h)$.

$\rho_{x x}, \rho_{x y}$ Magnetorresistividades longitudinal e transversal.

$\sigma \quad$ Polarização da luz. $\sigma= \pm 1$.

$\sigma_{z} \quad$ Operador de Pauli na direção $\hat{z}\left(\sigma_{z}= \pm 1\right)$. Usado como índice de spin.

$\sigma_{x x}, \sigma_{x y}$ Magnetocondutividades longitudinal e transversal.

$\zeta \quad$ Polarização, ou magnetização relativa, do gás de elétrons. 


\section{Capítulo 1}

\section{Introdução}

Fenômenos quânticos dependentes de spin se manifestam claramente em heteroestruturas semicondutoras com materiais semimagnéticos. Estes são compostos do grupo II-VI nas quais impurezas magnéticas (Mn, spin 5/2) são incorporadas na ligas. Os elétrons nestes sistemas são fortemente acoplados com os elétrons localizados da camada $3 d$ das impurezas de $\mathrm{Mn}$ via a interação de troca $s p-d$. Na presença de um campo magnético externo este acoplamento funciona como um potencial dependente de spin, levantando a degenerescência de spin dos estados de elétrons e buracos. Este efeito Zeeman efetivo induzido por troca sp-d é duas ordens de magnitude maior que o efeito ordinário e, portanto, consiste na essência dos efeitos dependentes de spin em semicondutores semimagnéticos.

Estruturas multicamadas de compostos semimagnéticos possibilitam a modulação contínua e dependente de spin dos offsets das bandas eletrônicas. Esta característica, mais a modulação intrínseca da heteroestrutura, proporciona meios convenientes para preparar/ajustar as propriedades eletrônicas do sistema. A super-rede de spin idealizadas por von Ortenberg [1], e subsequentemente realizada por Dai et al. [2] e Chou et al. [3], exemplifica bem estas ideias. Em particular, nesta super-rede não-dopada, segregação dependente de spin de portadores fotogerados ocorre na presença de campos magnéticos externos induzida pela interação de troca $s p$-d. Vários outras heteroestruturas quânticas não-dopadas e baseadas no Mn foram desenvolvidas e estudadas por espectroscopia magnetoóptica $[4-7]$.

A obtenção de altas densidades de portadores através de dopagem em heteroestruturas semimagnéticas do grupo II-VI [8] abriu uma nova e promissora área de pesquisa: magnetotransporte spin-polarizado. ${ }^{1}$ Por exemplo,

\footnotetext{
${ }^{1} \mathrm{O}$ magnetotransporte dependente de spin tem sua origem nas décadas de 70 e 80 em sistemas baseados em ligas ferromagnéticas. Neste contexto destacam-se os efeitos de magnetorresistência de tunelamento (TMR - tunnelling magnetoresistance) $[9,10]$ e a magnetorresistência gigante (GMR - giant magnetoresistance) [11]. Já em heteroestruturas com material semimagnético, dificuldades experimentais em obter altas dopagens adiou até meados da década de 90
} 
Smorchkova et al. [12] efetuaram medidas de efeito Hall quântico inteiro de um gás de elétrons bidimensional (2DEG) confinado em uma heteroestrutura digital magnética (DMH) [4] n-dopada. Por outro lado, Egues [13] investigou filtragem de spins em magnetotransporte longitudinal em DMHs. Neste contexto, a habilidade de controlar a polarização de spin de correntes eletrônicas é relevante para uma optoeletrônica dependente de spin [14-19].

Adicionalmente, uma série de experimentos recentes de magnetotransporte têm revelado uma física extremamente rica envolvendo 2DEGs no regime do efeito Hall quântico, tanto no regime inteiro, quanto no fracionário. Aqui eu destaco os efeitos de magnetotransporte relacionados com o surgimento de ferromagnetismo de efeito Hall (QHF) [20]. Estes experimentos [21-36] relatam o aparecimento de picos anômalos nas curvas da resistividade $\rho_{x x}$. Dependendo se o campo magnético aumenta ou diminui, os picos aparecem em valores diferentes de campo magnético, ou seja, ocorre histerese. Tanto o aparecimento do pico anômalo quanto a histerese ocorrem para temperaturas abaixo de uma temperatura crítica, sugerindo uma transição de fase. Também são efetuadas medidas de relaxação e de ruído associadas aos picos de magnetorresistência.

O trabalho aqui apresentado é uma investigação teórica da física dependente de spin que ocorre em DMHs. Mais especificamente, eu estudo o poço quântico digital magnético (DMQW), para o qual eu calculo a estrutura eletrônica na presença de um campo magnético externo, propriedades magnetoópticas, e magnetotransporte transversal (perpendicular à direção de crescimento). A física dependente de spin neste sistema é extremamente rica devido a, basicamente, dois fatores principais: (1) a interação de troca $s p-d$ confere à estrutura eletrônica do sistema uma forte dependência com o campo magnético e com o spin dos portadores; (2) ocorre quantização espacial ao longo da direção de crescimento, formando os 2DEGs. Em conjunto, estes dois fatores permitem explorar propriedades magnetoópticas, fenômenos de transporte relacionados ao efeito Hall quântico, e efeitos de muitos corpos presentes no 2DEG.

Existe uma extensa literatura abordando, separadamente, os vários ingredientes presentes nestes sistemas. Entretanto, pouca investigação teórica foi realizada abordando experimentos recentes $[4,7,21,37,38]$ que combinam o magnetismo do Mn, sua interação (troca sp-d) com os portadores, quantização espacial (2DEG) e orbital (níveis de Landau), tudo isto num regime em que efeitos de muitos corpos são relevantes - inclusive responsáveis pelo aparecimento de ferromagnetismo de efeito Hall (QHF) no sistema da Ref. [21]. A física dependente de spin induzida pela interação de troca $s p-d$ em DMHs é um campo com muitas possibilidades, abrangendo desde física básica até aplicações em spintrô-

a realização de magnetotransporte spin-polarizado [8]. 
nica (eletrônica resolvida em spin) e computação quântica - campos que muito se beneficiam quando há um estudo teórico paralelo ou até mesmo guiando os experimentos.

\subsection{Sistema "modelo" e resumo dos resultados}

Aqui eu investigo poços quânticos digitais magnéticos do grupo II-VI - ZnSe/ (Zn,Cd,Mn)Se [4, 7, 37, 38] e (Cd,Mg)Te/(Cd,Mn)Te [21]. Monocamadas contendo frações de Mn uniformemente espaçadas são substitucionalmente inseridas na sub-rede de cátions na região do poço quântico. Algumas das heteroestruturas são $n$-dopadas por modulação, sendo que as impurezas doadoras são separadas do poço por espaçadores intrínsecos. Estes sistemas são investigados na presença de um campo magnético externo aplicado na direção de crescimento das amostras. O DMQW é mais que sistema modelo para este trabalho, uma vez que toda esta investigação teórica é orientada para sistemas reais e com resultados experimentais recentes.

Nos sistemas não dopados [4, 7] eu investigo (i) o magnetismo intrínseco dos íons de Mn (aglomeração antiferromagnética), e (ii) a diluição do perfil de distribuição destas impurezas ao longo da amostra. Eu defino perfis modelo para descrever concentrações nominais e efetivas para o Mn ao longo da amostra e os utilizo para modelar, via potenciais efetivos, o efeito da interação de troca sp-d (Cap. 2). Eu então calculo o desdobramento de spin $\Delta E$ e o tempo de espalhamento de spin $\tau_{s f}$ oriundos da interação de troca sp-d (Cap. 3). Além de reproduzir os resultados experimentais para $\Delta E$ e $\tau_{s f}$, esta parte do trabalho complementa a abordagem teórica anterior [39].

Já nos sistemas dopados $[21,37,38]$ eu estudo magnetotransporte no regime de efeito Hall quântico (Caps. 4, 5 e 6). Eu começo calculando a dependência com o campo magnético da estrutura eletrônica e, em seguida, obtenho diversas grandezas de interesse experimental, a saber, densidades bidimensionais, magnetoresistividades transversais $\rho_{x x}$ e $\rho_{x y}$, e energia total. Eu calculo estas grandezas em função de diversos parâmetros: campo magnético (intensidade e ângulo), temperatura, densidade do 2DEG, concentração e distribuição do Mn. Os resultados para as grandezas citadas têm um acordo satisfatório com os respectivos experimentos. A saber, o comportamento com a temperatura das oscilações de Shubnikov-de Haas [37], o aparecimento de picos anômalos na magnetoresistividade $\rho_{x x}[21,37]$, e os ciclos de histerese que aparecem no magnetotransporte da Ref. [21]. 


\subsection{Abordagem teórica}

DFT/LSDA A abordagem teórica é baseada na teoria do funcional de densidade (DFT) [40], na sua versão dependente de spin na aproximação da densidade de spin local (LSDA) [41], formuladas no contexto da aproximação da massa efetiva [42-44]. Eu calculo a estrutura eletrônica do DMQW resolvendo as equações autoconsistentes de Kohn-Sham [41]. O potencial efetivo nestas equações é formado pelo potencial estrutural do poço quântico com barreiras com Mn, o potencial dependente de spin da interação de troca $s p$-d, e mais a contribuição de Coulomb do gás de elétrons confinado e das impurezas doadoras ionizadas (dopagem tipo $n$ ). Ao considerar as densidades de spin no cálculo da contribuição de troca-correlação da interação de Coulomb, eu não apenas incluo efeitos de muitos corpos no problema, mas também a sua dependência com o spin, essencial para, e.g., obter o ferromagnetismo de efeito Hall investigado no Cap. 6.

Troca sp-d A inclusão do efeito da interação de troca sp-d é feita na aproximação de campo médio, na qual se descreve a resposta paramagnética dos momentos de Mn via uma função de Brillouin efetiva [45]; esta interação entra na formulação via um potencial dependente de spin, modulando o perfil de potencial de elétrons e buracos em função do campo magnético externo.

Quantização de Landau Nos casos em que o campo magnético é intenso, a quantização de Landau é considerada. Enquanto a campo nulo, ou muito baixo, a relação de dispersão do movimento transversal é aproximadamente parabólica, na presença de um campo magnético a energia cinética colapsa em níveis (quase)discretos, denominados níveis de Landau (LLs). Eu considero este movimento orbital no plano $x y$ desacoplado do movimento na direção $z$ de crescimento, o que permite resolver separadamente cada parte do hamiltoniano eletrônico.

\subsection{Organização da tese}

Nas próximas seções deste capítulo introdutório eu faço um resumo da física dependente de spin em DMHs. Como este é o sistema "laboratório" para todos os tópicos investigados neste trabalho, aqui eu já introduzo os principais conceitos. Esta introdução é suficiente para abordar os tópicos dos capítulos iniciais, Caps. 2 e 3. Assim, eu adio para o Cap. 4 uma apresentação mais detalhada da física envolvendo 2DEGs sob o efeito de campos magnéticos intensos, bem como o 
resumo da abordagem teórica usando DFT/LSDA.

O texto desta tese está organizado em duas partes principais. A primeira parte enfoca a física dependente de spin relacionada ao magnetismo do Mn. Eu investigo como os efeitos de aglomeração antiferromagnética e diluição (por segregação e interdifusão) afetam as propriedades eletrônicas de DMHs. No Cap. 2 eu faço uma revisão da literatura sobre aglomeração e diluição, e termino propondo perfis modelos para as concentrações nominais e efetivas de Mn ao longo das heteroestruturas. Eu utilizo estes perfis nos demais capítulos para simular o potencial dependente de spin devido a interação de troca $s p-d$. No capítulo seguinte, Cap. 3, eu calculo o desdobramento de spin e o tempo de espalhamento de spin dos sistemas investigados experimentalmente nas Refs. [4, 7]. Com isto eu mostro que é necessário incluir simultaneamente os efeitos de aglomeração antiferromagnética e diluição para reproduzir os resultados experimentais.

$\mathrm{Na}$ segunda parte da tese eu mudo o enfoque para a física dependente de spin dos elétrons do 2DEG formado em heteroestruturas dopadas [21, 37, 38]. O Cap. 4 estabelece o modelo teórico que eu utilizo nos capítulos subsequentes para calcular a estrutura eletrônica e as propriedades de magnetotransporte. Usando este modelo, eu investigo no Cap. 5 o sistema de $n$-ZnSe/(Zn,Cd,Mn)Se experimentalmente estudado nas Refs. [37, 38], e no Cap. 6 o sistema da Ref. [21] de $n$ - $(\mathrm{Cd}, \mathrm{Mg}) \mathrm{Te} /(\mathrm{Cd}, \mathrm{Mn}) \mathrm{Te}$.

No capítulo final eu discuto os resultados obtidos neste trabalho, apontando a sua contribuição e relevância para a área. Eu indico também várias possibilidades que podem ser exploradas.

\subsection{Heteroestruturas digitais magnéticas}

Heteroestrutura digital magnética (DMH - digital magnetic heterostructure) é uma estrutura semicondutora na qual arranjos planares de átomos magnéticos são incorporados na região de confinamento. Em geral há vários destes arranjos uniformemente espaçados. A Figura 1.1 ilustra um poço quântico digital magnético com elementos do grupo II-VI. A liga semicondutora hospedeira é um cristal de ZnSe. Na região central, átomos de Cd são aleatoriamente inseridos na sub-rede de cátions substituindo uma fração de átomos de Zn. Como o gap entre as bandas de valência e de condução do ZnSe é maior que da liga ternária $\mathrm{Zn}_{1-y} \mathrm{Cd}_{y} \mathrm{Se}$, o resultado é a formação de uma região de estados confinados dentro das bandas do semicondutor, como ilustrado na Fig. 1.2. Os elétrons que eventualmente ocupam os estados confinados têm o seu movimento na direção de crescimento congelado, mas continuam livres no plano transversal. Esta é a realização prática do problema acadêmico do poço quântico. 


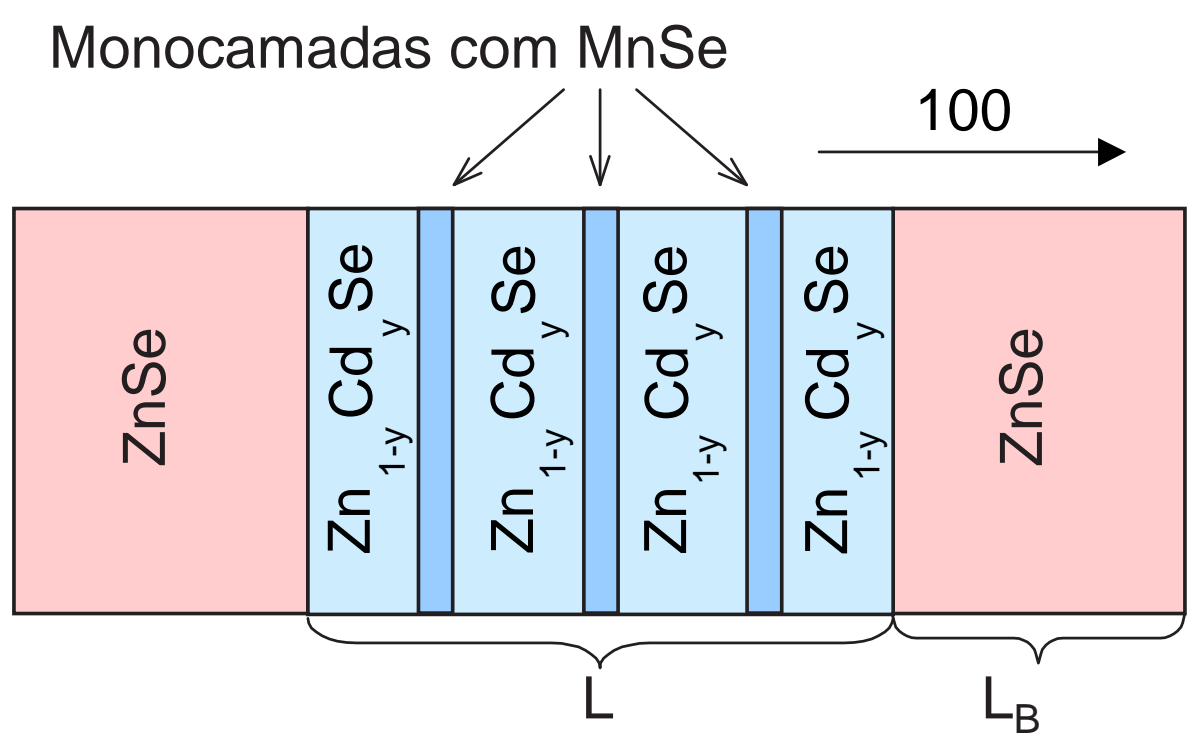

Figura 1.1: Exemplo de uma heteroestrutura digital magnética do grupo II-VI. Aqui o sistema é um poço quântico cuja liga principal é o ZnSe [direção de crescimento (100)]. A região do poço é aquela com uma fração y de Cd. O gap nesta região é menor que nas regiões vizinhas (barreiras), possibilitando a formação de estados ligados. Algumas monocamadas do poço contêm frações de MnSe, inseridas usando a técnica digital. O Mn interage fortemente com os portadores via a interação de troca $s p$ - $d$, acarretando um desdobramento de spin gigante na estrutura eletrônica.

As DMHs são originárias dos semicondutores magnéticos diluídos (DMS - diluted magnetic semiconductor), nos quais impurezas magnéticas são distribuídas uniformemente em regiões extensas da amostra. Nas DMHs os átomos de Mn também são aleatoriamente incorporados na sub-rede de cátions, mas em arranjos planares com espessura típica de uma monocamada. À técnica de se inserir frações bem determinadas de impurezas magnéticas em monocamadas específicas deu-se o nome de "técnica digital", o que explica o termo digital no nome desta classe de heteroestruturas. ${ }^{2}$ No caso da ilustração da Fig. 1.1, todos os sítios de cátions de três monocamadas foram completamente preenchidos por Mn. Normalmente apenas uma fração $x$ de sítios recebe os íons magnéticos, formando uma liga quaternária do tipo $\mathrm{Zn}_{1-x-y} \mathrm{Cd}_{y} \mathrm{Mn}_{x}$ Se naquelas monocamadas.

A principal característica destes sistemas é a forte interação que surge entre os momentos magnéticos localizados das impurezas e os portadores presen-

\footnotetext{
${ }^{2}$ Crooker et al. [4] utilizaram a técnica digital para inserir monocamadas com frações de Mn nas suas amostras, as quais denominaram DMHs. O termo "digital" refere-se ao fato do fluxo de Mn ser mantido constante e o controle da concentração ser feito simplesmente abrindo e fechando a célula de efusão [46]. A contrapartida "analógica" consiste em variar o fluxo de Mn durante o crescimento. A técnica crescimento epitaxial alternando a composição na escala de monocamadas atômicas vem da década de $70 \mathrm{com}$ o trabalho pioneiro de Gossard et al. [47].
} 


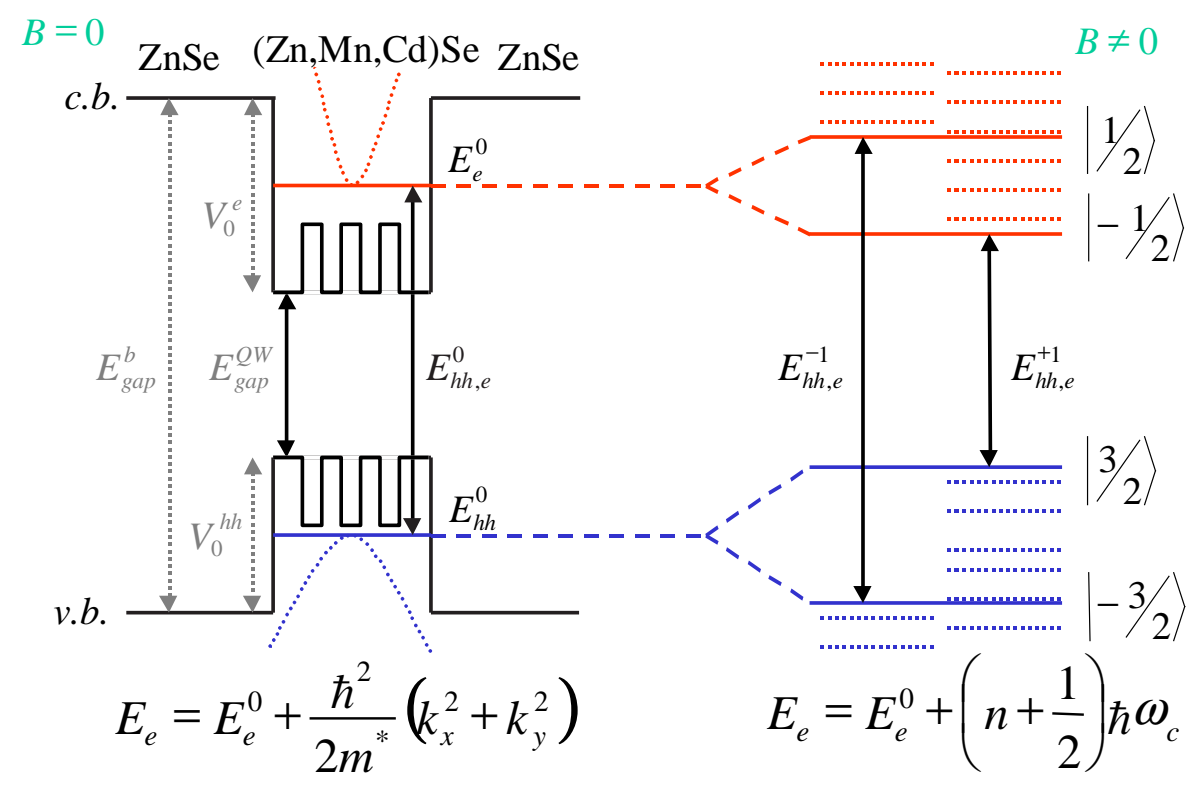

Figura 1.2: Diagrama da estrutura de bandas em um poço quântico digital magnético e o desdobramento de spin gigante devido a interação de troca $s p-d$. Em uma heteroestrutura como da Fig. 1.1, os perfis das bandas de condução (elétrons) e de valência (aqui apenas buracos pesados) possuem descontinuidades que originam regiões com estados ligados na direção de crescimento, mas com movimento livre na direção transversal (relação de dispersão parabólica). A origem destas descontinuidades está nos diferentes gaps das sucessivas regiões (painel $B=0$ ). $\mathrm{Na}$ presença de um campo magnético, os elétrons e buracos interagem com os momentos magnéticos alinhados dos íons $M n$ via a interação de troca $s p$-d, acarretando um desdobramento de spin gigante nas sub-bandas $(B \neq 0)$. O campo magnético também quantiza o movimento transversal, originando os chamados níveis de Landau. Em experimentos de fotoluminescência são registradas as transições elétron-buraco a campo nulo $E_{e, h h}^{0}$ e com campo aplicado $E_{e, h h}^{ \pm 1}$.

tes nas bandas de condução e de valência. Chamada de interação de troca $s p-d$, ela surge na presença de um campo magnético externo que alinha os momentos localizados dos íons de Mn. A magnitude desta iteração chega a ser algumas ordens de magnitude superior ao efeito Zeeman ordinário, acarretando um desdobramento de spin gigante nos níveis de energia, como ilustrado na Fig. 1.3.

Nos próximos parágrafos eu discuto a engenharia de bandas relacionada com a construção de heteroestruturas. Em seguida, eu apresento a física Mn nestas DMHs e a sua influência na estrutura eletrônica dos portadores.

\subsubsection{Engenharia de bandas no grupo II-VI}

As DMHs e DMSs são compostos ternários (e.g. $\mathrm{Cd}_{1-x} \mathrm{Mn}_{x} \mathrm{Te}$ ) e quaternários (e.g. $\mathrm{Zn}_{1-x-y} \mathrm{Cd}_{y} \mathrm{Mn}_{x} \mathrm{Se}$ ), frequentemente do grupo II-VI, com impurezas magnéticas ocupando parcialmente uma das sub-redes da liga principal. A estrutura de 


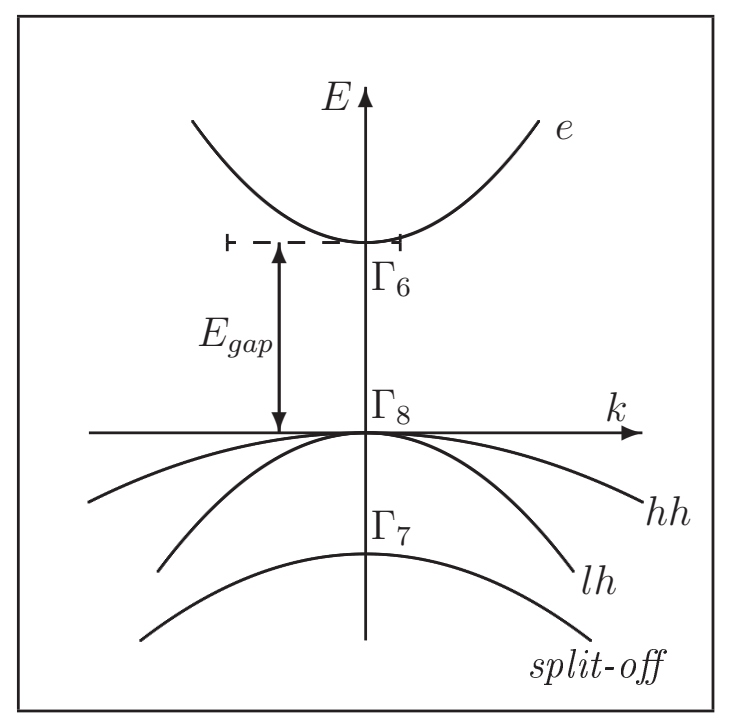

Figura 1.3: Esquema da estrutura de banda ao redor do ponto $\Gamma$ para uma uma estrutura do tipo zinc blend. A banda dos elétrons de condução $(e)$ é a banda $\Gamma_{6}$. A a interação spin-órbita é responsável pelo desdobramento da banda de valência em duas bandas, $\Gamma_{8}$, com buracos pesados $(h h)$ e leves $(l h)$, e $\Gamma_{7}$ (split-off).

banda dos semicondutores do grupo II-VI é caracterizada pelos 8 elétrons mais externos de cada célula unitária. Os elétrons das camadas fechadas internas são localizados e não contribuem para as propriedades ópticas e de transporte investigadas neste trabalho. Os dois elétrons dos cátions (simetria $s$ ) e os seis dos anions (simetria $p$ ) hibridizam, formando orbitais ligantes e anti-ligantes (modelo $s-p^{3}$ ). No sólido estes orbitais formam as bandas: os níveis ligantes $s$ são extremamente profundos e sempre ocupados por dois elétrons; os 6 elétrons restantes ocupam três orbitais ligantes do tipo $p$, que formam a banda de valência; já os orbitais anti-ligantes de simetria $s$ formam a banda de condução. Estes sistemas do grupo II-VI têm gap direto no centro da zona de Brillouin (ponto $\Gamma$ ), Fig. 1.3. A banda de condução é denominada $\Gamma_{6}$. A banda de valência é composta de dois níveis, o $\Gamma_{8}$, com buracos pesados e leves, e o $\Gamma_{7}$ que é a banda split-off.

Diferentes ligas binárias, e.g. $\mathrm{A}^{I I} \mathrm{C}^{V I}$ e $\mathrm{B}^{I I} \mathrm{C}^{V I}$, têm diferentes gaps, parâmetros de rede, etc. O mesmo ocorre quando, durante o crescimento, substituise uma fração dos elementos de uma liga binária por um ou mais elementos do mesmo grupo, formando ligas ternárias $\mathrm{A}_{1-x} \mathrm{~B}_{x} \mathrm{C}$ ou quaternárias $\mathrm{A}_{1-x-y} \mathrm{~B}_{x} \mathrm{C}_{y} \mathrm{D}$. Nos semicondutores semimagnéticos, as impurezas magnéticas, como por exemplo o Mn ([Ar $\left.] 4 s^{2} 3 d^{5}\right)$, são substitucionalmente inseridas na matriz semicondutora. Apesar de não pertencerem ao grupo II, o orbital $d$ é profundo e inerte do ponto de vista de estrutura eletrônica. ${ }^{3}$ Assim, para todos os efeitos, o Mn "funciona"

\footnotetext{
${ }^{3} \mathrm{O}$ nível de energia correspondente ao orbital $3 d$ preenchido fica $\sim 3.5 \mathrm{eV}$ abaixo do topo da banda de valência, e o nível não-ocupado fica também $\sim 3.5 \mathrm{eV}$ acima do topo da banda de valência [48]. A energia para uma transição envolvendo estes orbitais é muito alta, fora da escala dos efeitos que estou interessado.
} 
como um elemento do grupo II na formação das bandas. As heteroestruturas são formadas justamente por uma sucessão de camadas, cada uma com diferentes ligas e concentrações. A engenharia de bandas consiste em escolher adequadamente os materiais e concentrações para construir sistemas com as características estruturais e eletrônicas desejadas.

A rigor, as ligas ternárias e quaternárias não formam uma estrutura cristalina pois os elementos constituintes são distribuídos aleatoriamente nas sub-redes. Consequentemente, não existe simetria de translação necessária para definir funções de onda de Bloch, bem como os conceitos usuais de massa efetiva, função envelope, etc. Entretanto, a chamada aproximação do cristal virtual (VCA - virtual crystal approximation) frequentemente pode ser utilizada para descrever os estados eletrônicos. Nesta aproximação, o potencial aleatório dos diversos elementos é substituído por um potencial médio periódico, no qual a contribuição de cada elemento é pesada pela sua concentração na liga. Nesta aproximação a simetria de translação é recuperada. Como consequência, por exemplo, os gaps entre as bandas de condução e de valência de ligas ternárias $\mathrm{A}_{1-x} \mathrm{~B}_{x} \mathrm{C}$ interpolam (quase-)linearmente entre os valores dos gaps das ligas binárias $\mathrm{AC}$ e BC, i.e.,

$$
E_{\text {gap }}^{A_{1-x} B_{x} C}=(1-x) E_{\text {gap }}^{A C}+x E_{\text {gap }}^{B C}
$$

No poço quântico da Fig. 1.1, as barreiras são sistemas binários, enquanto o poço é uma liga terciária. Dentro do poço foram crescidas monocamadas quaternárias contendo Mn. Aqui eu determino o perfil de potencial estrutural nas bandas considerando justamente o efeito das diferentes composições nas alturas dos gaps de cada região. Estas diferenças entre as energias dos gaps é dividida entre as bandas de condução e de valência. Para se ter uma ideia de como fica esta divisão, pode-se usar como guia a regra do anion comum. Como o anion Se é comum em todas as camadas, e é ele que fornece os seis elétrons que formam a banda de valência, esta banda não é muito afetada. Assim, boa parte das diferenças de energia dos gaps fica no deslocamento da banda de condução ( $C B O$ conduction band offset; valores na literatura variam de $54 \%$ a $90 \%$ [49]).

Além do efeito sobre o gap, as diferentes composições também alteram elementos estruturais, como o parâmetro de rede. Isto acarreta strain ao longo da amostra. Neste trabalho eu não considero explicitamente esta contribuição e utilizo um valor único para o parâmetro de rede ao longo de todo o sistema. ${ }^{4}$

\footnotetext{
${ }^{4} \mathrm{Eu}$ não considero strain "diretamente" no hamiltoniano do sistema. Entretanto, ele está presente de duas formas. Primeiro, strain é responsável pelo desdobramento entre buracospesados $(h h)$ e buracos-leves $(l h)$ na base da banda de valência (degenerados na Fig. 1.3). Segundo, para as estruturas baseadas em ZnSe eu uso os resultados de Liaci et al. [50] para os
} 
Exemplo: potencial estrutural no poço quântico No caso da Fig. 1.1 há regiões com $\mathrm{ZnSe} \mathrm{Zn}_{0.8} \mathrm{Cd}_{0.2} \mathrm{Se}$, e $\mathrm{Zn}_{1-x-y} \mathrm{Mn}_{x} \mathrm{Cd}_{y} \mathrm{Se}$. Os gaps das ligas binárias fundamentais são [51, 52]:

$$
E_{\text {gap }}^{Z n S e} \cong 2.8 \mathrm{eV} \quad, \quad E_{\text {gap }}^{C d S e} \cong 1.8 \mathrm{eV} \quad, \text { e } \quad E_{\text {gap }}^{M n S e} \cong 3.5 \mathrm{eV}
$$

de forma que na região do poço quântico tenho

$$
E_{\text {gap }}^{Z n_{0.8} C d_{0.2} S e}=0.8 E_{\text {gap }}^{Z n S e}+0.2 E_{\text {gap }}^{C d S e} \cong 2.6 \mathrm{eV}
$$

Assim, a diferença de gap na interface $\mathrm{ZnSe} / \mathrm{Zn}_{0.8} \mathrm{Cd}_{0.2} \mathrm{Se}$ é $\cong 200 \mathrm{meV}$. Este valor é então dividido entre a banda de condução $v_{0}^{e} \cong 160 \mathrm{meV}$ e de valência $v_{0}^{h h} \cong 40 \mathrm{meV}(C B O=80 \%[53]) .^{5}$

Aqui eu admito que posso usar o mesmo procedimento para calcular a contribuição de monocamadas contendo Mn para o perfil de potencial estrutural. A diferença entre a energia do gap no $\mathrm{Zn}_{0.8} \mathrm{Cd}_{0.2} \mathrm{Se}(2.6 \mathrm{eV})$ e no MnSe $(3.5 \mathrm{eV})$ é de aproximadamente $0.9 \mathrm{eV}$. Seguindo a escolha da Ref. [39], divido esta diferença em $v_{b}^{e}=0.8 \mathrm{eV}$ na banda de condução e $v_{b}^{h h}=0.1 \mathrm{eV}$ na banda de valência. No caso do MnSe diluído com concentração molar $x$, as descontinuidades de potencial nas interfaces $\mathrm{Zn}_{0.8} \mathrm{Cd}_{0.2} \mathrm{Se} / \mathrm{MnSe}$ são dadas por $v_{b}^{e} x$ e $v_{b}^{h h} x$ (VCA).

\subsubsection{Magnetismo do Mn}

As impurezas de Mn inseridas em semicondutores do grupo II-VI entram substitucionalmente na sub-rede de cátions. A configuração eletrônica do $\mathrm{Mn}$ é $[\mathrm{Ar}] 4 s^{2} 3 d^{5}$, e são os elétrons mais externos da camada $4 s^{2}$ que participam da formação das bandas. O orbital $3 d^{5}$ semi-preenchido possui todos os spins alinhados no mesmo sentido (regra de Hund), gerando um spin permanente $S=5 / 2$, com $2 S+1$ componentes $S_{z}=-S,-S+1 \cdots S$. Do ponto de vista da estrutura eletrônica, estes elétrons são inertes pois há um custo muito grande em energia para mudar a orientação de um spin em relação aos demais. ${ }^{6}$

Sendo o momento orbital do Mn nulo, o momento angular total é igual ao spin total $S=5 / 2$. O momento magnético é então $\mu=-g_{M n} \mu_{B} \boldsymbol{S}$, cuja energia na presença de um campo magnético $\boldsymbol{B}=B \hat{z}$ é $-\mu \cdot \boldsymbol{B}=g_{M n} \mu_{B} B S_{z}$. O

offsets das bandas, valores estes que já contêm contribuição de strain.

${ }^{5}$ Como mencionado na nota 4 , nos cálculos deste trabalho eu uso o resultado de Liaci et al. [50] para as estruturas de ( $\mathrm{Zn}, \mathrm{Cd}) \mathrm{Se}$, que determinou os offsets das bandas usando espectroscopia magnetoóptica. São eles $v_{0}^{e}=0.925 y \mathrm{eV}$ e $v_{0}^{h h}=0.435 y \mathrm{eV}$, sendo $y<0.22$ a concentração de Cd. Usando estes valores os offsets do exemplo do texto ficariam $v_{0}^{e} \cong 185 \mathrm{meV}$ e $v_{0}^{h h} \cong 87 \mathrm{meV}$. A vantagem destes valores obtidos à partir de ajustes com o experimento é que já têm embutido outras contribuições, como por exemplo o strain.

${ }^{6}$ Veja a nota de rodapé 3 . 
spin médio de cada íon num ensemble a temperatura $T$ é

$$
\left\langle S_{z}\right\rangle=\frac{\sum_{S_{z}=-S}^{S} S_{z} \exp \left(-g_{M n} \mu_{B} B S_{z} / k_{B} T\right)}{\sum_{S_{z}=-S}^{S} \exp \left(-g_{M n} \mu_{B} B S_{z} / k_{B} T\right)}=-S B_{S}\left(\frac{g_{M n} \mu_{B} S B}{k_{B} T}\right)
$$

no qual $B_{S}$ é a função de Brillouin de spin $S$,

$$
B_{S}(x)=\frac{2 S+1}{2 S} \operatorname{coth} \frac{2 S+1}{2 S} x-\frac{1}{2 S} \operatorname{coth} \frac{1}{2 S} x .
$$

Portanto, a magnetização de uma coleção de $N$ íons paramagnéticos restritos num volume $V$ é dada por

$$
M=\frac{N}{V} g \mu_{B}\left\langle S_{z}\right\rangle=-g \mu_{B} N_{0} x S B_{S}\left(\frac{g_{M n} \mu_{B} S B}{k_{B} T}\right)
$$

na qual a densidade de $\mathrm{Mn} N / V$ foi reescrita como $N_{0} x$, sendo $N_{0}$ o número de cátions por unidade de volume e $x$ é a fração molar de Mn na sub-rede de cátions. ${ }^{7}$ As Eqs. (1.4) e (1.5) são válidas quando a concentração de Mn é muito baixa, num regime em que não ocorrem interações antiferromagnéticas. Entretanto, mesmo para concentrações maiores, (1.4) e (1.5) continuam fenomenologicamente úteis, desde que se substitua o spin e a temperatura por valores efetivos, i.e., $S \rightarrow \bar{S}(x)$ e $T \rightarrow T+T_{0}(x)$ [45]. Eu retomo este assunto no Cap. 2 no qual discuto a questão da aglomeração antiferromagnética presente em semicondutores semimagnéticos.

\subsubsection{Interação de troca $\mathrm{sp-d}$}

Os portadores livres no sistema interagem fortemente com os elétrons localizados na camada $3 d^{5}$ na chamada interação de troca $s p$ - $d$ ( $s$ - $d$ dos elétrons de condução com simetria $s$ com os elétrons localizados $d$, e $p$ - $d$ dos buracos na banda de valência com simetria $p$ com os elétrons localizados $d$ ). Esta interação pode ser descrita pelo seguinte hamiltoniano de Heisenberg $[54]^{8}$

$$
H_{s p-d}=-\frac{1}{2} \sum_{\boldsymbol{R}_{j}} J\left(\boldsymbol{r}-\boldsymbol{R}_{j}\right) \boldsymbol{S}_{j} \cdot \sigma
$$

em que $J$ representa a interação de troca entre o spin $S_{j}$ localizado na coordenada $\boldsymbol{R}_{j}$ e o spin $\sigma$ do portador.

A Eq. (1.6) não possui simetria translacional de um cristal perfeito.

\footnotetext{
${ }^{7}$ A fração molar $x$ de Mn é também chamada de concentração molar de Mn, ou simplesmente concentração de Mn.

${ }^{8}$ Apesar a literatura da área frequentemente se referir ao hamiltoniano (1.6) como sendo do tipo de Heisenberg, o mais apropriado seria dizer que ele é do tipo Kondo. Enquanto o primeiro geralmente refere-se à interação de spins isolados, o segundo descreve a interação do spin de uma impureza com os spins dos portadores estendidos na banda.
} 
Para evitar esta dificuldade a aproximação do cristal virtual (VCA - virtual crystal approximation) é usualmente empregada [51]. Para os portadores, que são estendidos e "vêem" muitas impurezas, tanto faz se estas impurezas estão distribuídas aleatoriamente ou se cada sítio da rede de cátions contribui com uma fração de momento magnético. Desta forma, a VCA é utilizada atribuindo a cada sítio da sub-rede de cátions uma contribuição $x J(\boldsymbol{r}-\boldsymbol{R})$ para o potencial, com $x$ a fração de impurezas magnéticas nos sítios dos cátions e a soma sendo feita sobre todos os $\boldsymbol{R}$ 's.

Em VCA, a distribuição das impurezas passa a ser periódica, mas não necessariamente a orientação do spin de cada impureza magnética. Portanto, o potencial ainda não tem a periodicidade da rede. Para superar esta dificuldade utiliza-se a aproximação do campo molecular: novamente, como os elétrons vêem várias impurezas, substitui-se o spin $\boldsymbol{S}_{j}$ de cada uma por uma média térmica $\langle\boldsymbol{S}\rangle$. Para o caso do campo magnético na direção $\hat{z}$ e uma amostra isotrópica, cada sítio da rede de cátions vai contribuir $\left\langle S_{z}\right\rangle$ para o spin total, enquanto $\left\langle S_{x}\right\rangle=\left\langle S_{y}\right\rangle=0$. Assim, em campo médio (MF), o hamiltoniano de troca fica

$$
H_{s p-d}^{M F}=-\frac{1}{2} x\left\langle S_{z}\right\rangle \sigma_{z} \sum_{\boldsymbol{R}} J(\boldsymbol{r}-\boldsymbol{R}) \rightarrow\left\{\begin{array}{c}
-\frac{1}{2} x\left\langle S_{z}\right\rangle \sigma_{z}^{e} N_{0} \alpha \\
\frac{1}{2} x\left\langle S_{z}\right\rangle \sigma_{z}^{h h} N_{0} \beta
\end{array},\right.
$$

no qual a soma é feita sobre todos os sítios $\boldsymbol{R}$ da sub-rede de cátions.

A passagem para o último termo da Eq. (1.7) consiste em reescrever o termo $H_{s p-d}^{M F}$ na aproximação de massa efetiva e função envelope, tanto para os elétrons (e) na banda de condução $\Gamma_{6}$ e para os buracos-pesados $(h h)$ na banda de valência $\Gamma_{8}$ (Fig. 1.3). Como a periodicidade da rede foi recuperada (VCA), é possível usar as mesmas funções de base que usualmente descrevem a estrutura de bandas do material. Denotando por $|s\rangle$ e $|p\rangle$ as funções de Bloch na bandas de elétrons e buracos-pesados, respectivamente, tem-se $\langle s|J| s\rangle=\alpha$ e $\langle p|J| p\rangle=\beta$, chamadas de integrais de troca [55]. Os termos $N_{0} \alpha$ e $N_{0} \beta$ são conhecidos para diversos materiais ( $N_{0}$ é a densidade de cátions). No $\mathrm{Zn}_{1-x} \mathrm{Mn}_{x}$ Se, por exemplo, as constantes de troca são $N_{0} \alpha=0.26 \mathrm{eV}$ para elétrons e $N_{0} \beta=-1.11 \mathrm{eV}$ para buracos-pesados [48]. A magnitude destas constantes já dá uma ideia de quão grande é o efeito da interação de troca $s p-d$. Para $x=0.01$, ela ainda é duas ordens de magnitude maior que a interação Zeeman ordinária, esta da ordem de $\mu_{B} \sim 0.06 \mathrm{meV} \mathrm{T}^{-1}$. 


\subsubsection{Estrutura eletrônica dependente de spin em DMHs}

\section{Potencial magnético}

A equação (1.7) é a contribuição para a energia de cada portador ( $e$ ou $h h$ ) devido a interação troca $s p-d$ no caso bulk. No caso das heteroestruturas digitais magnéticas, os íons de Mn são depositados em (mono)camadas específicas, Fig. 1.1. Assim, enquanto a distribuição do Mn é aleatória no plano transversal, há um perfil de concentração muito bem definido na direção de crescimento. Como a energia da interação de troca depende da sobreposição da função de onda eletrônica com os momentos localizados, o potencial correspondente segue o perfil de concentração do Mn.

Considerando o caso ideal em que cada monocamada magnética possui uma concentração planar $x_{p}$ de íons de $\mathrm{Mn}$, sem que haja nenhum tipo de diluição, posso descrever o perfil de concentração usando um modelo de barreiras quadradas. Utilizando a função de Heaviside (escada) $\Theta(z)$ e definindo uma função perfil quadrada como [39]

$$
\wp_{\mathrm{sqr}}\left(z ; N_{b},\left\{Z_{j}\right\}, d\right)=\sum_{j=1}^{N_{b}} \Theta\left(z-Z_{j}+\frac{d}{2}\right) \Theta\left(-z+Z_{j}+\frac{d}{2}\right)
$$

que descreve $N_{b}$ barreiras com largura $d$ localizadas nas coordenadas $Z_{j},{ }^{9}$ o perfil de densidade de Mn é simplesmente $x_{p} \wp_{\text {sqr }}(z)$ e o potencial de troca sp-d dependente de spin, e.g. para elétrons, fica

$$
v_{\mathrm{s}-\mathrm{d}}^{\sigma_{z}}(z ; B, T)=-\frac{1}{2} x_{p} \wp_{\mathrm{sqr}}(z)\left\langle S_{z}\right\rangle \sigma_{z} N_{0} \alpha .
$$

No Capítulo 2 eu vou reavaliar a adequação do potencial da Eq. (1.9) na descrição de DMHs reais. O propósito daquele capítulo é justamente mostrar que efeitos tanto de aglomeração antiferromagnética das impurezas de Mn, quanto de diluição do perfil de concentração destas impurezas, são essenciais para entender os resultados experimentais disponíveis. Para tanto eu aprimoro o modelo expresso em (1.9) para incluir estes efeitos, a saber, (i) ao invés de concentrações nominais $x_{p}$ eu utilizo concentrações efetivas que consideram a aglomeração antiferromagnética, e (ii) substituo o perfil quadrado da Eq. (1.8) por perfis diluídos por segregação ou interdifusão.

\footnotetext{
${ }^{9} \mathrm{Na}$ estrutura zinc blend de $(\mathrm{Zn}, \mathrm{Cd}, \mathrm{Mn}) /$ Se o parâmetro de rede é $a \sim 6 \AA$ e, portanto, a largura da monocamada é $d=a / 2 \sim 3 \AA$.
} 


\section{Potencial estrutural}

Eu também posso usar a função perfil quadrado (1.8) para descrever o perfil de potencial estrutural discutido na Sec. 1.4.1. Para o caso do perfil de um poço quântico de largura $L$ e centrado na coordenada $Z_{1}=0$, eu uso a função $1-\wp_{\text {sqr }}\left(z ; N_{b},\left\{Z_{j}\right\}, d\right)$. Sendo $v_{0}^{\Lambda}$ os offsets das bandas $(\Lambda=e, h h)$, o perfil de potencial estrutural pode ser escrito simplesmente por

$$
v_{0}^{\Lambda}(z)=v_{0}^{\Lambda}\left[1-\wp_{\mathrm{sqr}}(z ; 1,0, L)\right]
$$

com o zero de energia fixado na base de cada banda.

O perfil estrutural das barreiras contendo Mn pode ser descrito da mesma forma. Para o caso de $N_{b}$ barreiras magnéticas, cada uma com concentração planar $x_{p}$ de Mn, largura $d$, e coordenada $Z_{j}$, o perfil de potencial fica

$$
v_{b}^{\Lambda}(z)=v_{b}^{\Lambda} x_{p} \wp_{\mathrm{sqr}}\left(z ; 1,\left\{Z_{j}\right\}, d\right)
$$

Somando as contribuições estruturais do poço quântico (1.10) e das barreiras com Mn (1.11), o perfil de potencial estrutural resultante fica

$$
v_{0}(z)+v_{b}(z)=v_{0}^{\Lambda}\left[1-\wp_{\mathrm{sqr}}(z ; 1,0, L)\right]+v_{b}^{\Lambda} x_{p} \wp_{\mathrm{sqr}}\left(z ; 1,\left\{Z_{j}\right\}, d\right) .
$$

A Fig. 1.4 ilustra este potencial somado ao potencial dependente de spin (1.9) para o caso de elétrons. Na região contendo as impurezas magnéticas, elétrons com spins opostos sentem perfis de potencias diferentes devido a dependência da interação de troca $s-d$ com o spin.

\section{Hamiltoniano eletrônico}

O hamiltoniano eletrônico (apenas os portadores, $\Lambda=e, h h$, com massa efetiva $\left.m_{\Lambda}\right)$ é

$$
H=-\frac{\hbar^{2} \nabla^{2}}{2 m_{\Lambda}}+\boldsymbol{V}_{\Lambda}(z)
$$

sendo $\boldsymbol{V}_{\Lambda}$ o operador correspondente ao potencial dependente de spin $v(z)=$ $v_{0}(z)+v_{b}(z)+v_{s p-d}^{\Lambda}(z)$. Aqui eu ignorei, por hora, os termos do efeito Zeeman ordinário e a quantização de Landau para ressaltar o papel da energia de troca $s p-d$ na estrutura eletrônica. De fato, para campos relativamente baixos $(B \lesssim$ $1 \mathrm{~T}$ ) esta é uma boa aproximação; no decorrer deste trabalho eu adiciono estas contribuições de acordo com o regime estudado. Note, também, que os elétrons das impurezas de Mn não estão explícitos em $H$; em (1.13) a contribuição destes está embutida na forma do potencial efetivo de troca $s p-d$. 


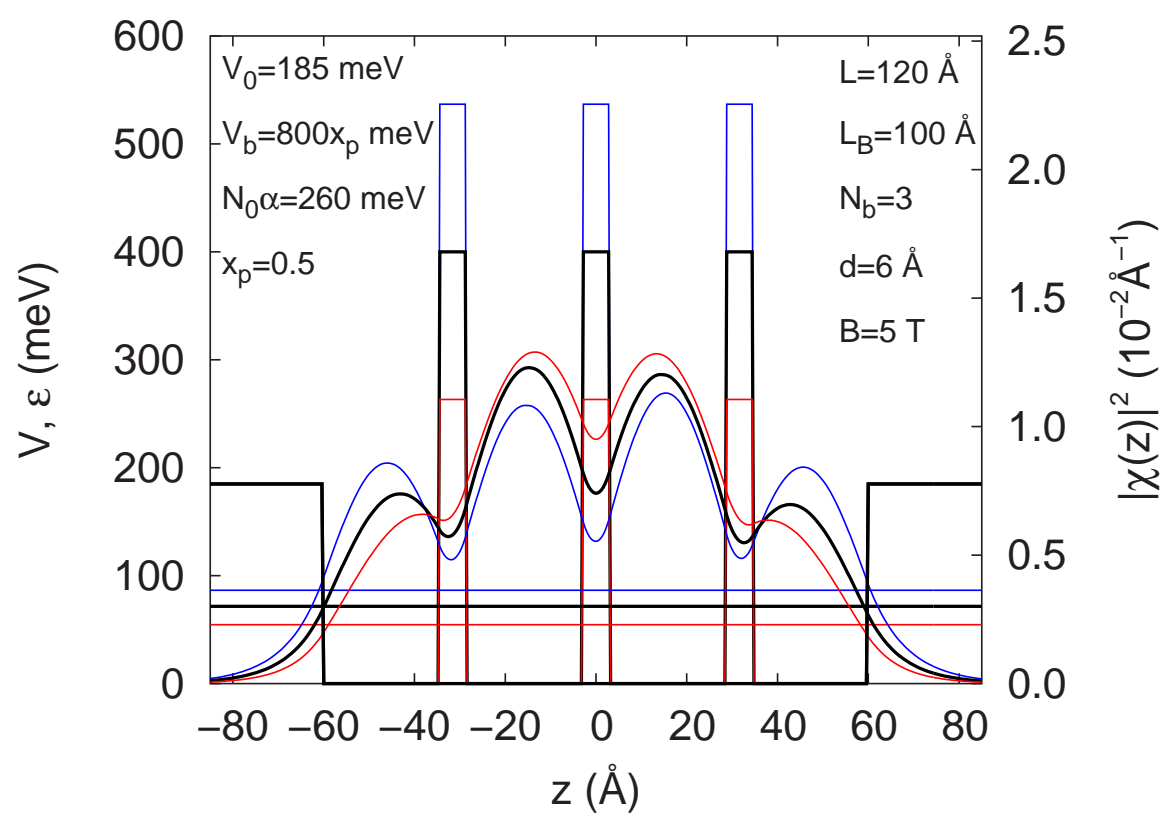

Figura 1.4: Perfil de potencial, sub-bandas e funções de onda de um poço quântico digital magnético como da Fig. 1.1, sujeito a um campo magnético externo (curvas coloridas). O perfil de potencial é dependente de spin nas regiões das barreiras com Mn. Os elétrons $|\downarrow\rangle$ sentem um potencial menor (vermelho) que os $|\uparrow\rangle$ (azul). Consequentemente (i) as sub-bandas de spin (linhas horizontais) sofrem um desdobramento de spin e (ii) as funções de onda são moduladas diferentemente. As curvas pretas correspondem a situação $\operatorname{com} B=0$.

\section{Sub-bandas dependentes de spin}

O hamiltoniano (1.13) é separável nas direções longitudinal $\hat{z}$ e transversal. Como a dependência espacial do potencial está apenas na direção de crescimento da amostra, no plano transversal o movimento eletrônico é livre. Assim, é necessário resolver apenas a equação de Schrödinger longitudinal

$$
\left[-\frac{\hbar^{2}}{2 m_{\Lambda}} \frac{\partial^{2}}{\partial z^{2}}+v_{\sigma_{z}}^{\Lambda}(z)\right] \chi_{i, \sigma_{z}}^{\Lambda}(z)=\varepsilon_{i, \sigma_{z}}^{\Lambda} \chi_{i, \sigma_{z}}^{\Lambda}(z),
$$

cuja solução fornece a função de onda (envelope) $\chi_{i, \sigma_{z}}^{\Lambda}(z)$ e a energia de sub-banda $\varepsilon_{i, \sigma_{z}}^{\Lambda}$ correspondentes ao movimento na direção $z$. A função de onda total é

$$
\psi_{i, \boldsymbol{k}_{\|}, \sigma_{z}}^{\Lambda}(\boldsymbol{r})=\frac{\mathrm{e}^{\mathrm{i} \boldsymbol{k}_{\|} \cdot \boldsymbol{r}_{\|}}}{\sqrt{A}} \chi_{i, \sigma_{z}}^{\Lambda}(z)\left|\sigma_{z}\right\rangle
$$

na qual $A$ é a área da seção transversal. A energia é

$$
\varepsilon_{i, \mathbf{k}_{\|}, \sigma_{z}}^{\Lambda}=\varepsilon_{i, \sigma_{z}}^{\Lambda}+\frac{\hbar^{2} k_{\Perp}^{2}}{2 m_{\Lambda}} .
$$


A Fig. 1.4 ilustra as soluções para a Eq. (1.14) para o caso de elétrons na banda de condução. O potencial dependente de spin é de uma DMH semelhante àquela da Fig. 1.1. As energias de sub-banda $\varepsilon_{i, \sigma_{z}}^{e}$ estão desdobradas devido a interação de troca $s$-d. Já as funções de onda $\chi_{i, \sigma_{z}}^{e}(z)$ estão moduladas refletindo o potenciais diferentes que elétrons $|\downarrow\rangle$ e $|\uparrow\rangle$ sentem.

Tridiagonalização numérica A resolução da equação de Schrödinger (1.14) é efetuada numericamente nesta tese utilizando o método das diferenças finitas [56]. O método consiste em discretizar a equação (1.14) e em seguida tridiagonalizar ${ }^{10}$ a matriz resultante $[57,58]$.

${ }^{10}$ Eu utilizo uma versão da rotina Givens [57] optimizada pelo Prof. L. N. Oliveira, IFSC/USP. 


\section{Parte I}

\section{Efeitos de Aglomeração e Diluição do Mn sobre as Propriedades Magnetoópticas em DMHs}





\section{Capítulo 2}

\section{Aglomeração Antiferromagnética e Diluição por Segregação e Interdifusão}

\section{Conteúdo}

As propriedades magnéticas de semicondutores semimagnéticos são fortemente dependentes do arranjo microscópico das impurezas magnéticas na liga hospedeira. Dois efeitos são predominantes: a aglomeração antiferromagnética entre momentos magnéticos vizinhos e a diluição das impurezas magnéticas ao longo da direção de crescimento, seja por segregação ou por interdifusão. Aqui eu faço uma revisão da literatura sobre estes dois efeitos e defino perfis de concentração nominal e efetiva para a distribuição de Mn. Estes perfis serão usados na formulação teórica dos demais capítulos deste trabalho.

2.1 Introdução . . . . . . . . . . . . . . . . . . 20

2.2 Aglomeração antiferromagnética e concentração efetiva . . . . . . 22

2.3 Diluição: segregação e interdifusão . . . . . . . . . . . . . . . . 27

2.4 Perfis modelo . . . . . . . . . . . . . . . . . . . . . . . . 32

2.5 Perfis de concentração: nominal e efetiva . . . . . . . . . . . 35

2.6 Discussões . . . . . . . . . . . . . . . . . . . . . . . . 42 


\subsection{Introdução}

As propriedades magnéticas de semicondutores semimagnéticos (bulk) dependem fortemente do arranjo microscópico das impurezas magnéticas na liga hospedeira. Dois efeitos são mais relevantes aqui [59]. (I) O efeito de aglomeração - também conhecido como efeito intrínseco - ocorre quando momentos magnéticos vizinhos próximos formam aglomerados antiferromagnéticos, acarretando uma diminuição na magnetização resultante. (II) Em contrapartida, há o efeito de diluição - chamado de efeito extrínseco - que ocorre quando o conteúdo magnético se "espalha" (dilui) para regiões vizinhas ao longo da amostra. Consequentemente, a distância média entre as impurezas magnéticas aumenta, o que faz com que a aglomeração antiferromagnética automaticamente diminua, aumentando a resposta paramagnética do sistema. ${ }^{1}$

Neste capítulo da tese eu analiso estes dois efeitos, que são essenciais para entender as propriedades magnéticas em DMS e DMH. As estruturas em questão são poços quânticos $\mathrm{ZnSe} /(\mathrm{Zn}, \mathrm{Cd}) \mathrm{Se}$ com arranjos planares periódicos de impurezas magnéticas (Mn, $S=5 / 2$ ), Fig. 1.1. Aqui eu considero perfis exponenciais e de função erro para simular a diluição de Mn. Este modelo será usado nos capítulos seguintes no cálculo a estrutura eletrônica para elétrons e buracos-pesados

Resultados experimentais de Crooker et al. [4], que eu analiso no próximo capítulo, apresentam evidências a favor da ideia de "quebra" dos pares antiferromagnéticos à medida que se aumenta a diluição do Mn. Esta visão também é corroborada por um cálculo teórico de Egues e Wilkins [39] utilizando barreiras quadradas ideais com espessura de uma monocamada (ML - monolayer) e concentração efetiva de Mn dentro do poço. No cálculo da Ref. [39], a inclusão de efeitos de aglomeração via uma concentração efetiva de Mn é crucial para obter acordo com dados experimentais.

Em um artigo de revisão sobre sistemas quânticos baseados em Mn, Awschalom e Samarth [60] mencionam que há muitas "evidências circunstanciais" sugerindo que efeitos de interdifusão e segregação nas interfaces de estruturas $\mathrm{ZnSe} /(\mathrm{Zn}, \mathrm{Mn})$ Se são limitados a escalas de comprimento de $1 \mathrm{ML}$. Estas evidências são: difração de raios X, oscilações RHEED, e estudos com dopagem $n^{2}$ Assim, uma descrição idealizada de DMHs em termos de arranjos quase-

\footnotetext{
${ }^{1}$ As denominações intrínseco e extrínseco se referem às propriedades magnéticas no seguinte sentido: A aglomeração antiferromagnética entre os momentos magnéticos das impurezas tem origem em interações entre os spins (troca d-d) e, portanto, é um efeito magnético intrínseco. Já a diluição tem a ver com a distribuição das impurezas na liga, o que acaba por afetar a aglomeração antiferromagnética indiretamente, e por isso denominado de efeito extrínseco [59].

${ }^{2}$ Arranjos planares aparentemente reduzem a formação de armadilhas (traps) para elétrons, possibilitando dopagens maiores que em sistemas quaternários com distribuição de Mn aleatória
} 
bidimensionais de íons de Mn pode não ser uma má primeira aproximação. Aqueles autores também notam que, ao contrário de sistemas (Cd,Mn)Te/CdTe, não há investigação magnetoóptica detalhada das interfaces em ZnSe/(Zn,Mn)Se - e consequentemente não existem evidências factuais - para embasar o modelo de configurações planares do Mn.

Entretanto, existe uma diferença qualitativa importante entre poços quânticos de (Cd,Mn)Te/CdTe e ZnSe/(Zn,Mn)Se presentes na literatura:

(Cd,Mn)Te/CdTe Nestes sistemas o Mn fica nas barreiras e a região do poço não é magnética. Nestes casos, apenas as extremidades da função de onda que penetram nas barreiras é que sentem o efeito magnético - e portanto, são sensíveis aos perfis de concentração de Mn nas interfaces. Estudos magnetoópticos recentes encontraram uma forte dependência do desdobramento de spin induzido por troca $s p-d$ com a ordem de crescimento das camadas magnéticas e não-magnéticas nestes sistemas [59, 61-66]. Interfaces invertidas [CdTe sobre (Cd,Mn)Te] apresentam desdobramento de spin mais acentuado que as chamadas estruturas normais [(Cd,Mn)Te sobre $\mathrm{CdTe}]$.

ZnSe/(Zn,Mn)Se O Mn está dentro do poço, onde as funções de onda eletrônicas cobrem praticamente toda a extensão. Nestas DMHs, a resposta magnetoóptica deve ser menos sensível aos detalhes do perfil de concentração de Mn. Isto porque a função de onda se sobrepõe a várias camadas magnéticas e apenas a resposta "integrada" do sistema é acessível experimentalmente.

Este fato não significa, entretanto, que a dimensão da distribuição de Mn dentro do poço não é relevante em DMHs. Pelo contrário, a ("quase-bi") dimensionalidade dos arranjos de Mn é muito importante quando efeitos de aglomeração são considerados; entretanto, isto não pode ser investigado "diretamente" com as técnicas magnetoópticas usuais. Assim, apesar da ausência de medidas diretas investigando a dimensionalidade das configurações de spin em DMHs, os resultados mencionados no parágrafo anterior indicam que os spins de Mn de fato formam arranjos quase bidimensionais no interior da região do poço [60].

Nesta parte da tese eu extendo o trabalho anterior de Egues e Wilkins [39] sobre fenômenos dependentes de spin em DMHs, incluindo efeitos de segregação e interdifusão das impurezas de Mn no cálculo do desdobramento de spin e do tempo de espalhamento de spin induzidos pela interação de troca sp-d. Eu sigo o trabalho da Ref. [59] e considero perfis modelos apropriados para a concentração de impurezas magnéticas. Adicionalmente, eu defino uma concentração

(bulk DMS). 
efetiva "contínua" que interpola entre o caso bulk e o estritamente bidimensional. Isto permite considerar a aglomeração de $\mathrm{Mn}$ em todos os perfis modelos de uma forma bastante direta. O resultado desta abordagem é que a introdução de perfis não-ideais e as correspondentes concentrações efetivas de Mn melhora os resultados dos cálculos anteriores [39], o que eu mostro no Cap 3.

\subsection{Aglomeração antiferromagnética e concentração efetiva}

Em semicondutores semimagnéticos bulk, só em concentrações baixas $(x \leqslant 0.01$, sendo $x$ a concentração molar de impurezas magnéticas) é possível considerar os momentos magnéticos isolados e com interações antiferromagnéticas desprezíveis [48]. A magnetização desta coleção de íons paramagnéticos segue a função de Brillouin (1.4) para spin $S=5 / 2$. Para concentrações maiores, a distância média entre momentos magnéticos vizinhos próximos (NN) diminui e, consequentemente, ocorre aglomeração antiferromagnética devido a interação de troca $d$ - $d$, $J_{N N} \sim-10 \mathrm{~K}$ [48]. A abordagem utilizando a função de Brillouin continua fenomenologicamente válida, mas com o spin $S=5 / 2$ e a temperatura $T$ substituídos por valores efetivos $\bar{S}(x)$ e $T_{\text {eff }}=T+T_{0}(x)[45] .^{3}$

Em um estudo sistemático do desdobramento de spin gigante em $\mathrm{Cd}_{1-x} \mathrm{Mn}_{x}$ Te, Gaj et al. [59] variaram a concentração de Mn até $x=0.67 \mathrm{e}$ parametrizaram o spin e a temperatura efetivos de maneira a reproduzir os dados experimentais (Fig. 2.1). Grieshaber et al. [61] usou esta mesma parametrização para escrever a magnetização como função da concentração molar $x$ de $\mathrm{Mn}$, do campo magnético $B$, e da temperatura $T$. Esta parametrização foi escrita como, em unidades de $g_{M n} \mu_{B}$,

$$
M_{b u l k}(x, B, T)=x \bar{S}(x) B_{5 / 2}\left(\frac{5}{2} \frac{g_{M n} \mu_{B} B}{k_{B}\left[T+T_{0}(x)\right]}\right),
$$

na qual

$$
\bar{S}(x)=S[0.265 \exp (-43.34 x)+0.735 \exp (-6.19 x)]
$$

é o spin efetivo das impurezas magnéticas, e

$$
T_{0}(x)=\frac{35.37 \mathrm{~K}}{1+2.752 x} x
$$

\footnotetext{
${ }^{3}$ Quando forma-se aglomerados antiferromagnéticos, o número de spins que de fato contribuem para a magnetização é menor, tal que o spin médio é $\bar{S}(x)<S$. Este antiferromagnetismo também é indicado pela temperatura efetiva $T_{0}(x)>0$. Quando há ferromagnetismo, $T_{0}$ é negativo.
} 


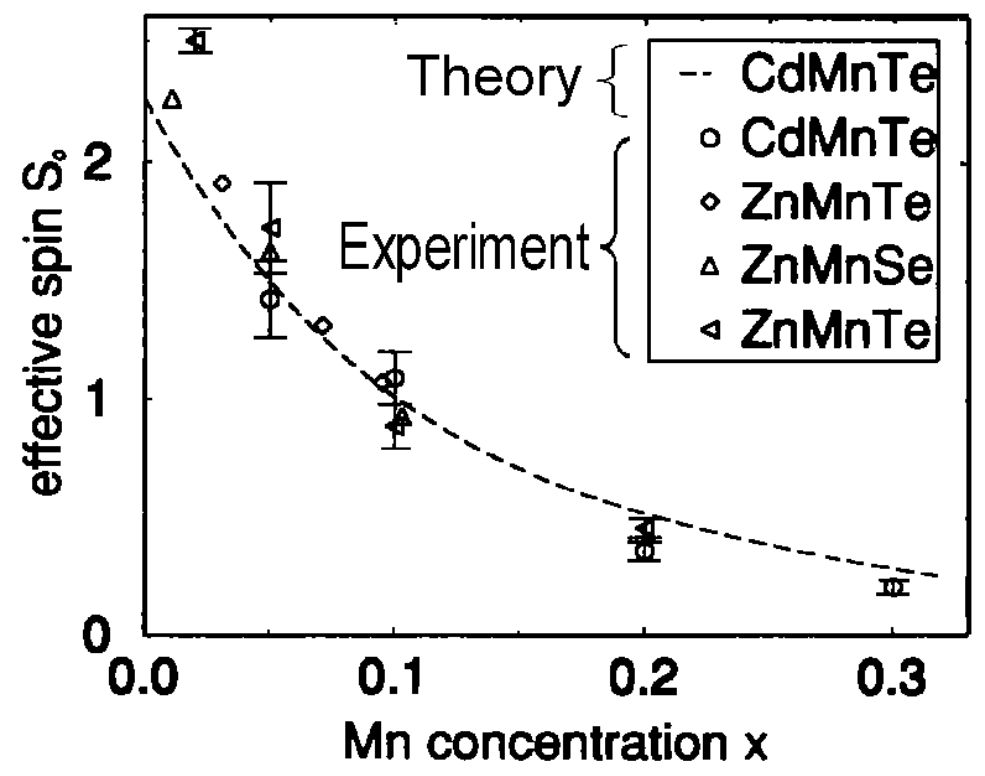

Figura 2.1: Valores experimentais para o spin efetivo em função da concentração de Mn para diversos semicondutores semimagnéticos (símbolos). A curva tracejada é a simulação numérica de Fatah et al. [67]. A parametrização de Gaj et al. [59], Eqs. (2.1-2.3), interpola os resultados experimentais para (Cd,Mn) Te ilustrados nesta figura. Baseado em Fatah et al. [67].

é uma correção fenomenológica para a temperatura.

Este resultado é válido não apenas para $\mathrm{Cd}_{1-x} \mathrm{Mn}_{x} \mathrm{Te}$, mas também para um classe inteira de semicondutores semimagnéticos do grupo II-VI $\left(\mathrm{A}_{1-x}^{I I} \mathrm{Mn}_{x} \mathrm{~B}^{V I}\right)$, como ilustrado na Fig. 2.1 [67]. A dependência do spin efetivo $\bar{S}$ dos íons de Mn com a sua fração molar $x$ é independente do hospedeiro não magnético. Este achado experimental foi reproduzido com sucesso por simulações numéricas em que Mn foi distribuído aleatoriamente na rede FCC de cátions e o número de íons primeiros vizinhos não emparelhados foi contado [67], linha sólida na Fig. 2.1.

Estatística de aglomerados Microscopicamente, o spin efetivo pode ser entendido em termos de estatística de aglomerados, que é válida desde que a distribuição dos íons magnéticos seja aleatória [69]. Para campos magnéticos suficientemente baixos, tal que os aglomerados estejam no estado fundamental, as contribuições para o spin total são [68, 70]: um átomo isolado de Mn contribui para a magnetização com um spin $S=5 / 2$; um par de spins não contribui pois fica alinhado antiparalelamente devido a interação antiferromagnética, $S^{P}=0$; tripletos abertos $(O)$ contribuem com $S^{O}=5 / 2$, de forma que a contribuição média por spin é $5 / 6$; tripletos fechados $(C)$ contribuem com $S^{C}=1 / 2$, e a contribuição média por spin é 1/6. A Fig. 2.2 mostra vários tipos de aglomerados possíveis. 

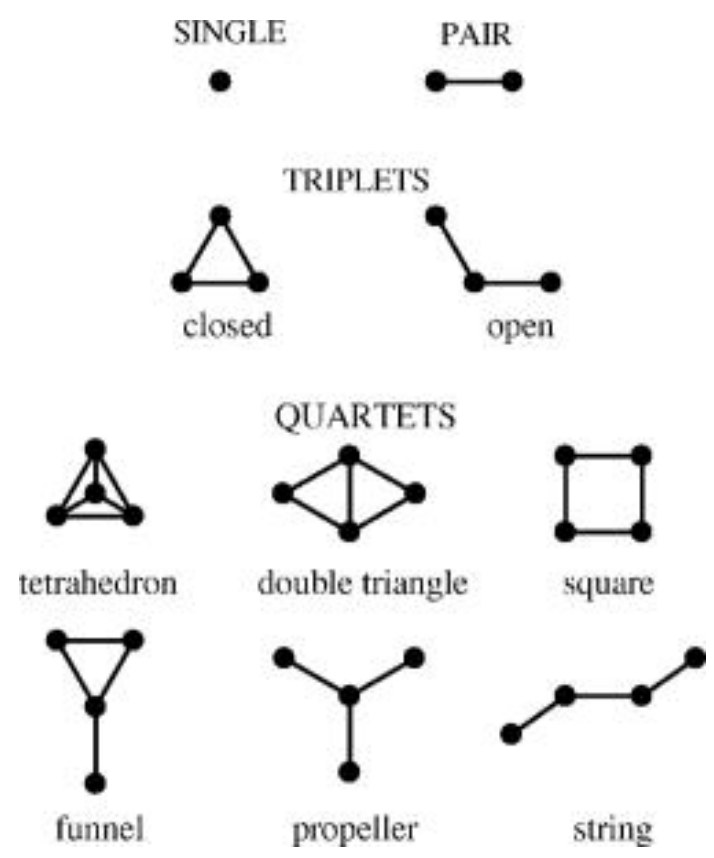

Figura 2.2: Tipos de aglomerados de íons magnéticos idênticos com apenas uma constante de troca. As linhas indicam as ligações de troca. Apenas aglomerados com até quatro spins são ilustrados. Existem dois tipos de tripletos (trímeros) e seis tipos de quartetos (tetrâmeros). Extraído de Shapira e Bindilatti [68].

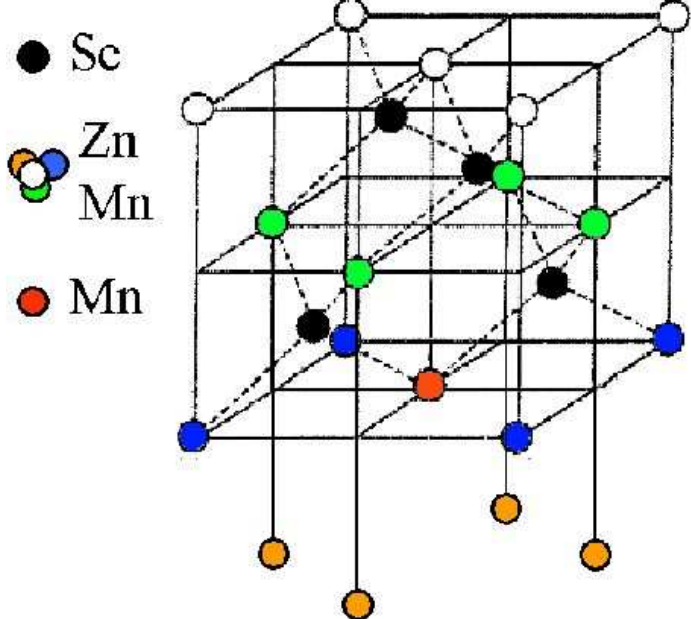

Figura 2.3: Estrutura cúbica (zinc blend) do semicondutor semimagnético (Zn,Mn)Se. O Mn substitui o Zn em alguns sítios da sub-rede (fcc) de cátions. Cada íon de Mn, e.g. o sítio vermelho, possui 12 vizinhos próximos, sendo 4 na sua mesma monocamada (azul) e quatro em cada monocamada adjacente (laranja e verde).

A probabilidade $P_{i}$ de encontrar cada configuração é função da concentração $x$ e da dimensionalidade. Veja na Fig. 2.3, por exemplo, como é a vizinhança de uma impureza de Mn na estrutura cúbica do semicondutor semimagnético $(\mathrm{Zn}, \mathrm{Mn})$ Se. A probabilidade de encontrar um átomo de Mn isolado em bulk é $P_{1}=(1-x)^{12}$, enquanto em uma interface ela é $P_{1}=(1-x)^{8}$, e em uma monocamada ela é $P_{1}=(1-x)^{4}$. A Tabela 2.1, reproduzida da Ref. [61], resume as probabilidades de encontrar aglomerados até tripletos em bulk, interface e monocamada ideais.

Usando a estatística de aglomerados, o spin normalizado médio, até tripletos abertos $(O)$ e fechados $(C)$, é dado por [71]

$$
\bar{S} / S=\left(S P_{1}+S^{O} P_{3}^{O}+S^{C} P_{3}^{C}\right) / S=P_{1}+\frac{1}{3} P_{3}^{O}+\frac{1}{15} P_{3}^{C},
$$

com $S=5 / 2$ para o Mn. Ao considerar valores maiores para $x$, aglomerados de maior ordem devem ser considerados, mas suas contribuições são sucessivamente menores. Kreitman et al. [70] sugeriram que um patamar superior para a contribuição de aglomerados de ordem superior poderia ser estimado supondo que (i) 
Tabela 2.1: Probabilidade $P_{i}$ que um átomo de Mn esteja em um aglomerado de $i$ spins na rede $\mathrm{FCC}$ de cátions da liga $\mathrm{A}_{1-x}^{I I} \mathrm{Mn}_{x} \mathrm{~B}^{V I}$, para bulk, interface, e uma monocamada. $y=1-x$. Baseado em Grieshaber et al. [61].

\begin{tabular}{cccc}
\hline \hline & Bulk & Interface & Monocamada \\
\hline$P_{1}$ & $y^{12}$ & $y^{8}$ & $y^{4}$ \\
$P_{2}$ & $12 x y^{18}$ & $4 x\left[y^{12}+y^{14}\right]$ & $4 x y^{6}$ \\
$P_{3}^{C}$ & $24 x^{2} y^{22}$ & $4 x^{2}\left[2 y^{16}+y^{18}\right]$ & 0 \\
$P_{3}^{O}$ & $18 x^{2}\left[2 y^{23}+5 y^{24}\right]$ & $2 x^{2}\left[6 y^{15}+5 y^{16}+8 y^{18}+3 y^{19}+10 y^{20}\right]$ & $6 x^{2}\left[2 y^{7}+y^{8}\right]$ \\
$P_{i>3}$ & {$\left[P_{1}+\frac{1}{3} P_{3}^{O}+\frac{1}{15} P_{3}^{C}\right]+\frac{1}{5}\left(1-P_{1}-P_{2}-P_{3}^{O}-P_{3}^{C}\right)$} \\
\hline \hline
\end{tabular}

quartetos quase não contribuem, e (ii) todos os demais spins formam quintetos que contribuem com $S=5 / 2$. Shapira et al. [71] então propuseram a seguinte correção para o spin efetivo

$$
\bar{S} / S=\left(P_{1}+\frac{1}{3} P_{3}^{O}+\frac{1}{15} P_{3}^{C}\right)+\left(1-P_{1}-P_{2}-P_{3}^{O}-P_{3}^{C}\right) / 5
$$

que melhorou a descrição dos resultados experimentais baseada na estatística de aglomerados até $x \simeq 0.1$ [Fig. 2.4(a)]. Para uma revisão aprofundada de estatística de aglomerados veja o trabalho de Shapira e Bindilatti [68] e suas referências.

Modelo alternativo (fenomenológico) de Grieshaber et al. [61] Uma descrição alternativa, e fenomenológica, para a magnetização local em interfaces e monocamadas foi proposta por Grieshaber et al. [61], que definiram uma concentração média

$$
x_{N N}(z)=\frac{x(z-d)+x(z)+x(z+d)}{3}
$$

que leva em consideração a concentração em monocamadas adjacentes ( $d$ é a largura da monocamada). Para os casos ideais bulk, interface e monocamada, esta concentração $x_{N N}$ é simplesmente $x, 2 x / 3$ e $x / 3$, respectivamente. A magnetização local na heteroestrutura é então escrita como

$$
M_{\text {local }}(x(z), B, T) \equiv \frac{M_{b u l k}\left(x_{N N}(z), B, T\right)}{x_{N N}(z)} x(z)
$$

na qual $M_{b u l k}(x, B, T)$ é dada pela parametrização das Eqs. (2.1)-(2.3), e $x(z)$ é o perfil de concentração molar nominal. Consequentemente, o spin efetivo neste modelo é simplesmente $\bar{S}\left(x_{N N}(x)\right)$ [cf. Eq. (2.1)]. 

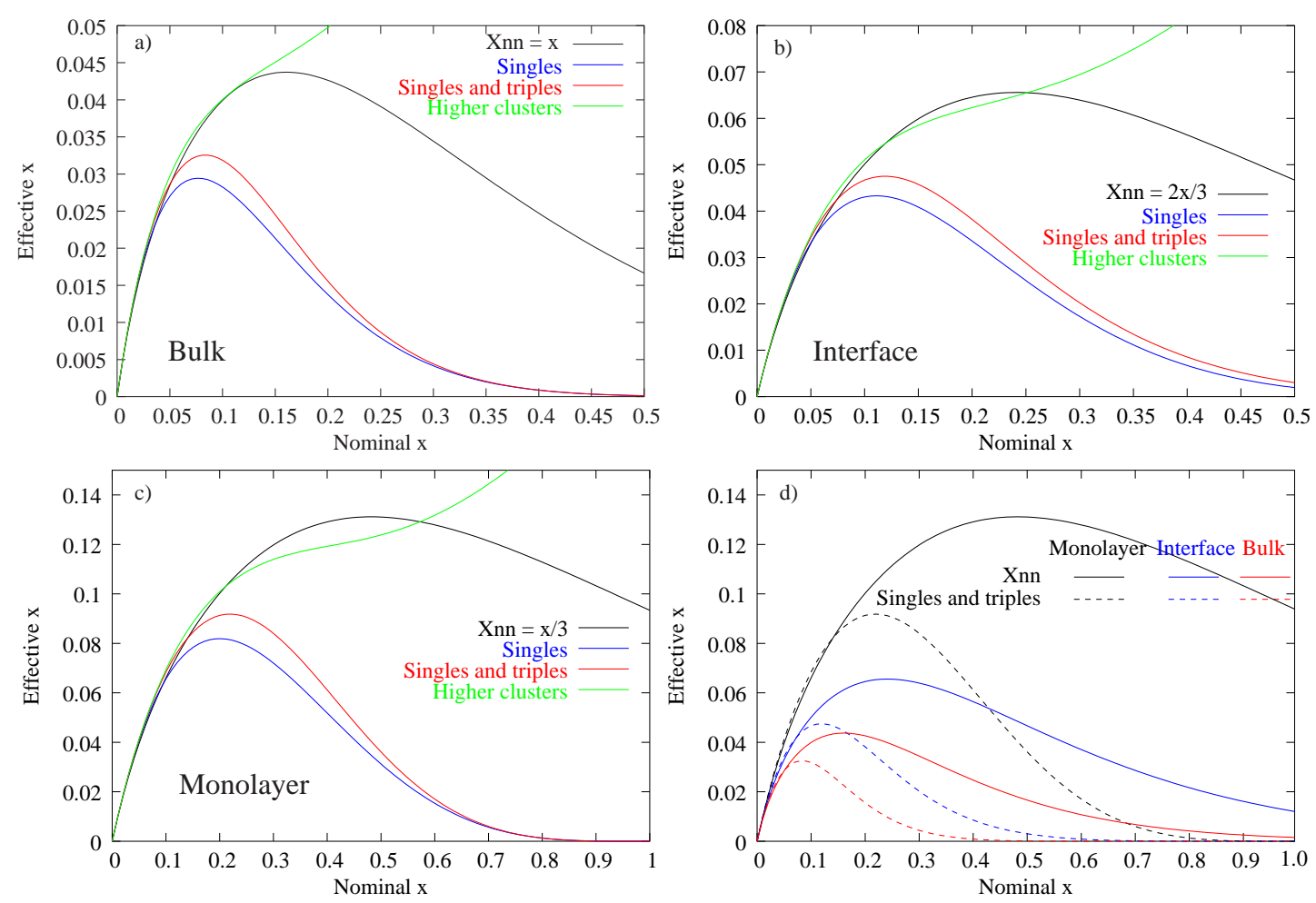

Figura 2.4: Comparação entre os modelos da estatística de aglomerados e o modelo fenomenológico de concentração média $x_{N N}$. Cada painel mostra a concentração efetiva $\bar{x}=x S^{-1} \sum_{i} P_{i} S_{i}$ para bulk (a), interface (b), e monocamada (c). Os modelos de aglomerados consideram íons isolados (azul), íons isolados mais tripletos (vermelho), e o modelo com correção para aglomerados de ordem maior $(i>3$, verde). No painel (d) os três casos são comparados na mesma escala.

Spin efetivo $\bar{S}$ e concentração efetiva $\bar{x}$ Note que o conceito de spin efetivo pode ser entendido da seguinte maneira: o produto $x \bar{S}(x)$ na Eq. (2.1) diz que todos os átomos de Mn estão alinhados e contribuem com um spin efetivo $\bar{S}(x)$ para a magnetização total. Isto é equivalente a dizer que existe apenas uma fração efetiva $\bar{x}(x)$, que é função de $x$ nominal, de íons de Mn alinhados que contribuem $\operatorname{com} S=5 / 2$ para a mesma magnetização total [71, 72], i.e.,

$$
x \bar{S}(x) \equiv \bar{x}(x) S
$$

Como será mostrado na Seção 2.4, este conceito de concentração efetiva permite que eu modele os perfis de potencial de uma maneira bastante intuitiva.

A Figura 2.4 compara (i) as concentrações efetivas obtidas com os modelos de estatística de aglomerados da Tabela 2.1, (ii) a correção proposta para aglomerados de ordem superior [Eq. (2.5)], e (iii) o modelo fenomenológico com concentração média $x_{N N}$. Para bulk, o modelo $x_{N N}$ reproduz exatamente por construção - os dados experimentais disponíveis para DMS do grupo II-VI para $x \lesssim 0.67$. Note que, quando se considera aglomerados de ordem maior no 
modelo de estatística de aglomerados, as concentrações efetivas se aproximam da curva "experimental" $\left(x_{N N}\right)$ da Fig. 2.4(a). A correção da Eq. (2.5) tornou esta melhoria possível para concentrações até $x \approx 0.1$. Para valores maiores de $x$, (i) o modelo de singletos e tripletos superestima a aglomeração de spins, diminuindo excessivamente a concentração efetiva, e (ii) a correção para aglomerados de ordem superior subestima aglomeração, sendo isto uma consequência direta da hipótese na Eq. (2.5) que todos os demais spins estão em aglomerados de cinco átomos.

Para os casos de interface e monocamada ideais, Figs. 2.4(b) e (c), $\bar{x}$ aumenta na sequência bulk $\rightarrow$ interface $\rightarrow$ monocamada, como ilustrado no painel comparativo (d). Isto acontece porque estas interfaces e monocamadas ideais possuem menos vizinhos nas camadas adjacentes que no caso bulk. Nestes casos, o modelo $x_{N N}$ não tem uma justificativa experimental, como em bulk, sendo então apenas um modelo que concorda com, e é justificado pela, estatística de aglomerados para $x \lesssim 0.25$.

\subsection{Diluição: segregação e interdifusão}

Heteroestruturas semicondutoras reais não possuem interfaces e monocamadas abruptas. Segregação e interdifusão são efeitos importantes que diluem o perfil de concentração de qualquer impureza substitucional, incluindo os íons magnéticos em DMS e DMH. Consequentemente, estes efeitos diminuem a aglomeração de spin, aumentando a resposta paramagnética do sistema. Assim, o meu interesse nesta parte do trabalho é explorar modelos mais realistas para as barreiras magnéticas em DMHs. Segregação e interdifusão de impurezas de Mn são investigadas usando modelos apropriados para construir o perfil de potencial usado no cálculo da estrutura eletrônica que eu faço nos capítulos seguintes.

\subsubsection{Segregação}

O processo de segregação, que ocorre durante o crescimento do cristal semicondutor, corresponde a troca de átomos entre a superfície sendo crescida e a monocamada imediatamente abaixo. Quando são inseridas impurezas em um cristal, por exemplo o Mn ocupando alguns sítios de cations no ZnSe, as impurezas não apenas ocupam alguns sítios na superfície em crescimento, como também trocam de posição com átomos da monocamada imediatamente anterior, como ilustrado na Fig. 2.5(a). 

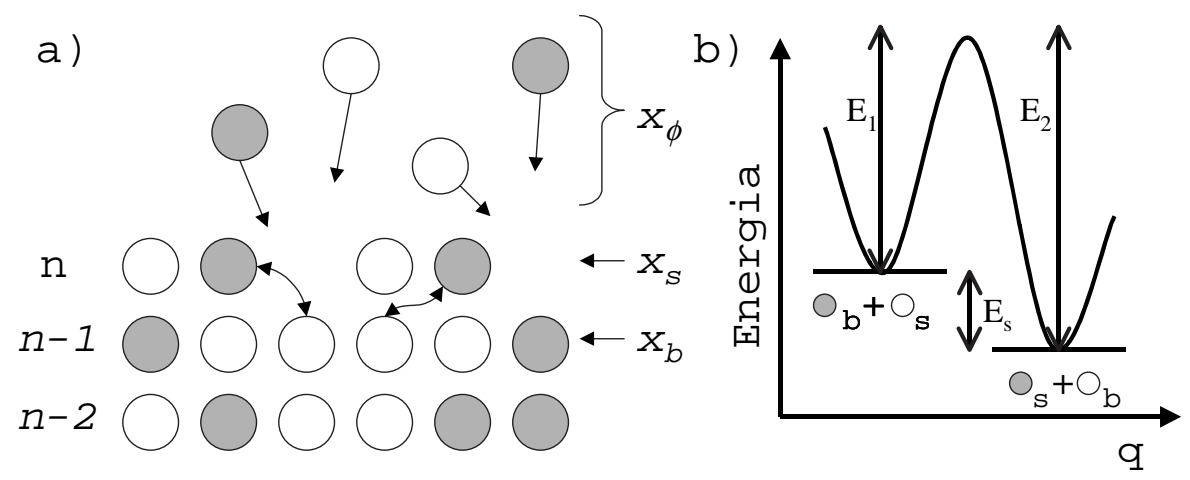

Figura 2.5: Ilustração do efeito de segregação durante o processo de crescimento de um semicondutor. (a) Durante a formação da $n$-ésima monocamada, átomos do cristal hospedeiro (o) e de impurezas $(\bullet)$ são depositados nesta camada. Na segregação ocorre a troca ente átomos da superfície e da última camada já crescida do bulk. As concentrações de impurezas na superfície $x_{s}$ e na última camada do bulk $x_{b}$ dependem tanto do fluxo incidente $x_{\phi}$ de impurezas, quanto da eficiência de segregação. (b) Esquema da energia para as combinações com as impurezas no bulk e na superfície num espaço de configurações $q$. Se a impureza vence a barreira de energia para sair do bulk e ir para a superfície, a configuração final neste exemplo é energeticamente mais favorável. A energia de segregação é definida como a diferença de energia entre as duas configurações. Painel (b) extraído de Dehaese et al. [73].

Modelo termodinâmico da segregação A troca de átomos entre a superfície e a última camada do bulk ocorre pois, devido a agitação térmica, as concentrações de impurezas na superfície $x_{s}$ e na última camada do bulk $x_{b}$ tendem a atingir um equilíbrio. Este processo é frequentemente descrito por um modelo termodinâmico que estabelece que, após o crescimento de cada monocamada, as concentrações de equilíbrio da superfície e do bulk obedecem a lei da ação das massas [74]

$$
\frac{x_{s}\left(1-x_{b}\right)}{x_{b}\left(1-x_{s}\right)}=\exp \left(\frac{E_{s}}{k_{B} T}\right) .
$$

Para entender esta equação considere o diagrama da Fig. 2.5(b). A concentração $x_{b}$ indica a probabilidade da impureza estar na última monocamada do bulk e a concentração $x_{s}$ indica a probabilidade da impureza estar na superfície. Assim, a probabilidade conjunta da impureza ficar na última monocamada do bulk é dada por $x_{b}\left(1-x_{s}\right) \propto \exp \left(E_{1} / k_{B} T\right)$, proporcional ao fator de Boltzmann associado à energia $E_{1}$. Já a probabilidade da impureza permanecer na superfície é $x_{s}(1-$ $\left.x_{b}\right) \propto \exp \left(E_{2} / k_{B} T\right)$, proporcional ao fator de Boltzmann com energia $E_{2}$. A razão entre estas probabilidades resulta na Eq. (2.9).

\footnotetext{
${ }^{4}$ As energias $E_{1}$ e $E_{2}$, que são energias de ligação da impureza na liga, são da ordem de eV; já a energia de segregação é tipicamente $E_{s} \sim 100 \mathrm{meV}$ [73-75]. As temperaturas de crescimento típicas são $T \sim 500 \mathrm{~K}$. Assim, o fator de Boltzmann para a impureza superar a barreira de energia é $\exp \left(-E_{1} / k_{B} T\right) \sim 10^{-11}$ ! Entretanto, os eventos ocorrem na frequência de vibração
} 
Se for energeticamente favorável que a impureza fique na superfície, isto é, a energia de segregação $E_{s}$ é positiva [veja a Fig. 2.5(b)], a troca de átomos irá favorecer a segregação de impurezas para a superfície $\left(x_{s}>x_{b}\right)$. Mesmo se $E_{s}=0$ - probabilidade da impureza sair da superfície e ir para o bulk é igual a probabilidade do percurso contrário -, o balanço de concentração fará que $x_{s}=x_{b}$, na situação chamada de intermixing.

Para exemplificar, considere justamente o caso de intermixing numa situação em que o fluxo de impurezas fornece uma concentração $x_{p}$ apenas durante o crescimento da monocamada $n$. Assim, quando o sistema entrar em equilíbrio, ${ }^{5}$ as concentrações serão necessariamente $x_{s}=x_{b}=x_{p} / 2$. Quando as próximas camadas são crescidas, mas com $x_{\phi}=0$, a concentração irá caindo pela metade sucessivamente, gerando um perfil

$$
x(m)=x_{p}\left(\frac{1}{2}\right)^{m}=x_{p} \exp \left(-\frac{m}{\ell_{o}}\right),
$$

sendo $\ell_{0}=1 / \ln 2 \simeq 1.442$ (monocamadas) o comprimento característico de segregação para o caso de intermixing. Este exemplo mostra que o processo de segregação faz com que a concentração fique com um perfil do tipo exponencial, com comprimento característico $\ell$ relacionado com a energia de segregação $E_{s}$. Entretanto, não há uma expressão simples que relacione $E_{s} \operatorname{com} \ell$; para $E_{s} \neq 0$ temos apenas que $\ell \neq \ell_{0}$.

A Eq. (2.9) fornece apenas a relação entre as concentrações finais da superfície $x_{s}$ e da última camada do bulk $x_{b}$ depois do crescimento de uma monocamada. Para efetivamente calcular as concentrações durante o processo de crescimento é necessário uma equação adicional que relaciona estas duas variáveis. A equação de conservação de massa

$$
x_{s}(n)+x_{b}(n)=x_{s}(n-1)+x_{\phi}
$$

serve para este propósito. Ela diz que a concentração de impurezas total, cuja concentração é dividida em $x_{s}$ e $x_{b}$ ao final do crescimento da $n$-ésima camada, é dada pela concentração superficial que segregou quando do crescimento da camada anterior, mais o fluxo de impurezas presente durante o crescimento da

da rede cristalina, que é de $10^{13} \mathrm{~s}^{-1}$ [73], indicando que, na escala de tempo de crescimento da amostra, há sim troca de átomos entre a superfície e o bulk.

${ }^{5}$ Neste modelo termodinâmico, considera-se que a duração do crescimento de cada monocamada leva o tempo necessário para que as concentrações entrem em equilíbrio, seguindo a lei da ação das massas. Existem também os chamados modelos cinéticos [73, 75, 76], em que não é necessário esperar o equilíbrio. Estes modelos são mais complicados e estão além do escopo deste trabalho. O aspecto importante aqui é que o tipo de perfil de concentração obtido por estes modelos é semelhante ao modelo termodinâmico, ou seja, o perfil é do tipo exponencial. 
camada $n$. Resolvendo as Eqs. (2.9) e (2.11) conjuntamente é possível obter os perfis de concentração de impurezas para quaisquer valor de $E_{s}$ e $x_{\phi}$ dentro do modelo termodinâmico.

\subsubsection{Interdifusão}

Devido a agitação térmica as impurezas podem se difundir pela amostra. Este processo, que pode ocorrer durante e após (annealing) o crescimento, também é responsável pela diluição das impurezas [59].

A difusão é regida pelas chamadas leis de Fick [77]. A primeira lei de Fick é

$$
J=-D \frac{\partial C}{\partial z}
$$

com J o fluxo de partículas por unidade de tempo e de área, D a constante de difusão com dimensão de área por tempo, e C o perfil de densidade de partículas. Esta lei diz que que o fluxo é proporcional ao gradiente de concentração. A segunda lei de Fick é a equação de difusão

$$
\frac{\partial C}{\partial t}=-\frac{\partial J}{\partial z}=D \frac{\partial^{2} C}{\partial z^{2}}
$$

O perfil de concentração resultante é obtido resolvendo a equação da segunda lei de Fick. Para exemplificar, nos parágrafos seguintes eu mostro como ficam os perfis de interdifusão para três casos relevantes: fonte discreta, fonte semi-infinita (interface) e fonte finita (camada)

Fonte discreta Considere que no instante $t=0$ há uma concentração $C=C_{0}$ na coordenada $x=0$. A solução para a segunda lei de Fick [Eq. 2.13] é

$$
C(x, t)=C_{0} \frac{1}{2 \sqrt{\pi D t}} \exp \left(\frac{-x^{2}}{4 D t}\right)=C_{0} \frac{1}{\ell \sqrt{\pi}} \exp \left(-\frac{x^{2}}{\ell^{2}}\right),
$$

sendo $\ell=2 \sqrt{D t}$ o comprimento característico de difusão. Veja a Fig. 2.6(a) que ilustra estes perfis gaussianos para três diferentes valores para $\ell$.

Fontes semi-infinitas (interface) O caso de uma fonte semi-infinita significa que, no instante $t=0$ inicial, o perfil de concentração é $C(x>0,0)=C_{0}$ e $C(x \leq 0,0)=0$. Para resolver o problema é possível considerar que cada plano situado numa coordenada $a_{i}$ sofre difusão, e que a concentração na coordenada $x$ é a soma das contribuições de todos os planos $\alpha_{i}$ com larguras $\Delta \alpha_{i}$ [Fig. 2.6(b)], 

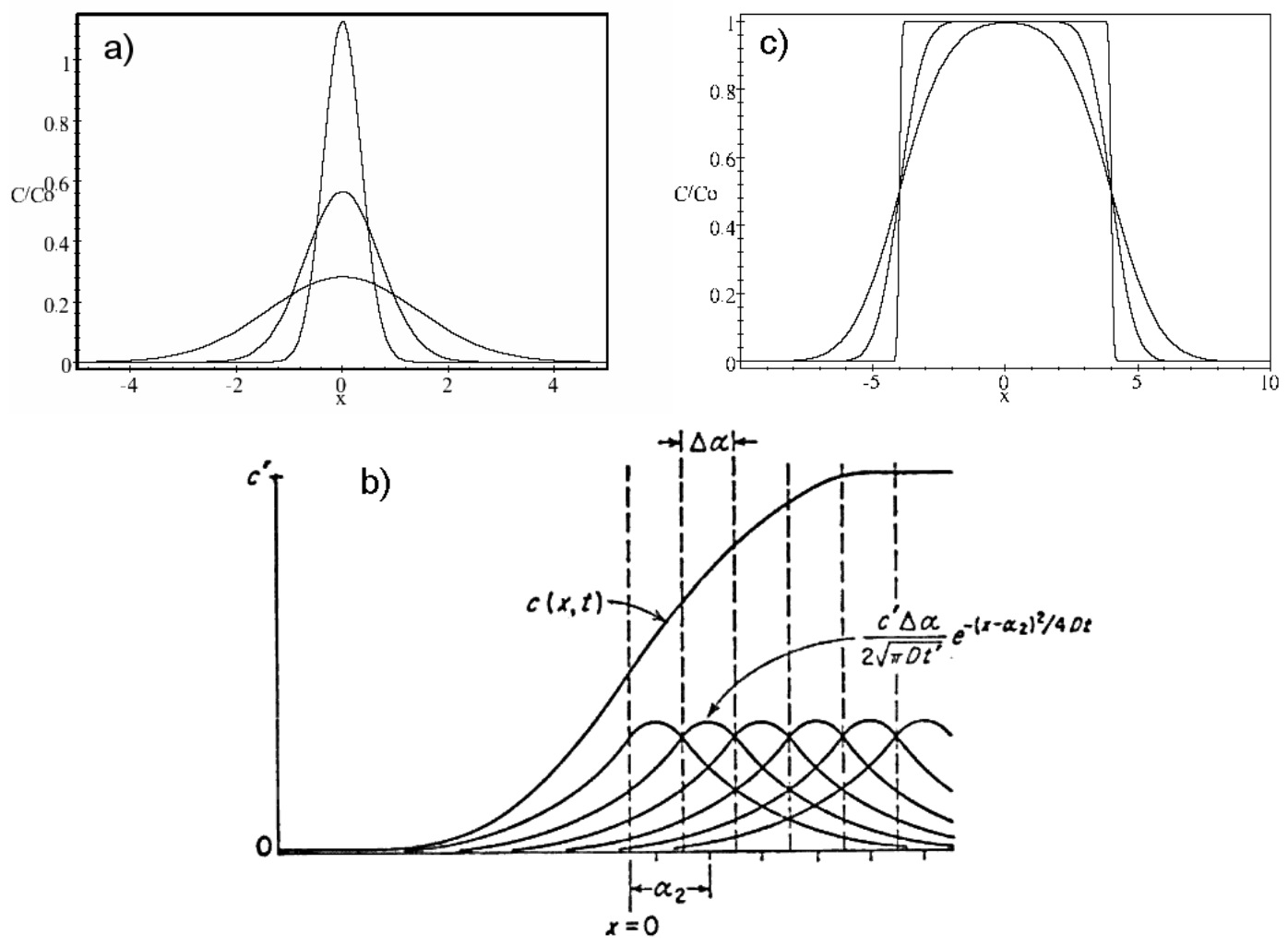

Figura 2.6: (a) Perfil de difusão proveniente de uma fonte planar com $C(x=0, t=$ $0)=C_{0}$. Os perfis mais largos correspondem a tempos de difusão maiores. Os comprimentos característicos usados são $\ell=0.5,1$ e 2. (b) Composição do perfil de concentração devido a difusão de uma fonte semi-infinita. O perfil resultante é a sobreposição dos perfis de cada camada de coordenada $\alpha$. A soma sobre todos os perfis resulta numa função $C(x, t)$ com o perfil de função erro. [P. G. Shewmon, Diffusion in Solids (McGraw, New York,1963), Fig. 1-5 ]. (c) Perfil de difusão proveniente de uma fonte extensa e finita, com largura $d=5$. Os perfis mais largos correspondem a tempos de difusão maiores. Os comprimentos característicos usados são $\ell=0.1,1$ e 2 .

ie.,

$$
C(x, t) \simeq \frac{C_{0}}{2 \sqrt{\pi D t}} \sum_{i=1}^{n} \Delta \alpha_{i} \exp \left[-\frac{\left(x-\alpha_{i}\right)^{2}}{4 D t}\right] .
$$

No limite contínuo em que $n \rightarrow \infty$ e $\Delta \alpha_{i} \rightarrow 0$, a somatória transforma-se em uma integral

$$
C(x, t)=\frac{C_{0}}{2 \sqrt{\pi D t}} \int_{0}^{\infty} \exp \left[-\frac{(x-\alpha)^{2}}{4 D t}\right] \mathrm{d} \alpha .
$$

Fazendo a substituição de variáveis $\eta=(x-\alpha) / 2 \sqrt{D t}$ a solução fica

$$
C(x, t)=\frac{C_{0}}{\sqrt{\pi}} \int_{0}^{x / 2 \sqrt{D t}} \exp \left[-\eta^{2}\right] \mathrm{d} \eta
$$


cuja integral pode ser escrita em termos da função erro

$$
C(x, t)=\frac{C_{0}}{2}\left[1+\operatorname{erf}\left(\frac{x}{2 \sqrt{D t}}\right)\right]=\frac{C_{0}}{2}\left[1+\operatorname{erf}\left(\frac{x}{\ell}\right)\right] .
$$

A Fig. 2.6(b) ilustra o perfil final para este caso de uma interface.

Fonte finita (camada) No caso de uma fonte finita (uma camada de largura d), considere que no instante inicial a distribuição de impurezas é dada por

$$
C(x, 0)=\left\{\begin{array}{cc}
C_{0} & -d / 2 \leq x \leq d / 2 \\
0 & |x|>d / 2
\end{array} .\right.
$$

O perfil de concentração no instante $t$ pode ser obtido de forma similar que o caso de fontes semi-infinitas, mas restrigindo a contribuição a apenas a largura $d$ da fonte. A integral da Eq. (2.16) fica então

$$
C(x, t)=\frac{C_{0}}{2 \sqrt{\pi D t}} \int_{-d / 2}^{d / 2} \exp \left[-\frac{(x-\alpha)^{2}}{4 D t}\right] \mathrm{d} \alpha .
$$

Refazendo os mesmo passos anteriores, i.e., substituição de variáveis $\eta=(x-$ $\alpha) / 2 \sqrt{D t}$, fico com a seguinte equação

$$
C(x, t)=-C_{0} \frac{1}{\sqrt{\pi}} \int_{\frac{x+d / 2}{2 \sqrt{D t}}}^{\frac{x-d / 2}{2 \sqrt{D t}}} \exp \left[-\eta^{2}\right] \mathrm{d} \eta
$$

cuja solução é

$$
C(x, t)=\frac{1}{2} C_{0}\left[\operatorname{erf}\left(\frac{x+d / 2}{\ell}\right)-\operatorname{erf}\left(\frac{x-d / 2}{\ell}\right)\right] .
$$

A Fig. 2.6(c) ilustra o perfil de difusão de uma camada para diferentes valores de comprimentos de interdifusão $\ell$.

\subsection{Perfis modelo}

Neste trabalho eu não estou interessado em investigar os aspectos microscópicos de diluição, sejam eles por segregação ou interdifusão. O que de fato eu preciso é uma prescrição prática para descrever o perfil de distribuição das impurezas magnéticas para, em conjunto com os modelos de aglomeração, simular a concentração efetiva de Mn. Esta, por sua vez, está relacionada com a magnitude da interação de troca $s p$ - $d$ entre os momentos das impurezas magnéticas e os portadores nas bandas de elétrons e buracos. O aspecto relevante aqui é o quanto a 
diluição por segregação ou interdifusão diminui a aglomeração, aumentando significativamente o spin efetivo quando comparado com o caso ideal sem diluição.

Na seção anterior eu mostrei que tanto a segregação quanto a interdifusão geram perfis de concentração característicos, exponencial e função-erro, respectivamente. Assim, vou utilizar estes perfis para construir funções de perf $i l$ que simulem a distribuição de Mn em DMHs semelhantes àquela da Fig. 1.1.

Aqui eu sigo, essencialmente, o trabalho de Gaj et al. [59], que utilizou perfis contínuos, tanto para o caso de segregação quanto para interdifusão. Este perfis consistem de expressões, contínuas na coordenada da direção de crescimento, para as interfaces entre regiões com conteúdos de impurezas diferentes. Note que, ao usar um perfil contínuo para descrever um sistema cuja variação de concentração está na escala de monocamadas atômicas, eu estou fazendo uma aproximação. Esta é uma opção prática do ponto de vista de implementação numérica do modelo e que, a julgar pelos resultados que apresento nos capítulos subsequentes, se mostrou bastante satisfatória.

Segregação Para o caso de segregação, eu descrevo a variação da concentração molar de $\operatorname{Mn}$ de $x=x_{p}$ a $x=0$ em uma interface por $x(z)=$ $x_{p} f(-z / \ell)$, sendo $f(z)$ definido por [59]

$$
f(z)=\left\{\begin{array}{ll}
1 & z \geq 1 \\
\exp (z-1) & z<1
\end{array} .\right.
$$

Usando esta definição, construo uma função de perfil contínua

$$
\wp_{\exp }(z)=\sum_{j=1}^{N_{b}}\left[f\left(\frac{\left(Z_{j}+\frac{d}{2}\right)-z}{\ell}\right)-f\left(\frac{\left(Z_{j}-\frac{d}{2}\right)-z}{\ell}\right)\right]
$$

que descreve $N_{b}$ monocamadas com largura $d$ localizadas nas coordenadas $Z_{j}$ ao longo do eixo de crescimento. ${ }^{6}$ O comprimento característico $\ell$, que está relacionado com a energia de segregação, é na prática o parâmetro que define a extensão da diluição das impurezas devido a segregação.

Interdifusão Usando a solução (2.22) da equação de difusão para o caso da fonte de impurezas ser uma camada, eu defino uma função de perfil

\footnotetext{
${ }^{6} \mathrm{O}$ perfil que a Eq. (2.24) fornece é ligeiramente deslocado com relação ao perfil do modelo termodinâmico. Isto pode ser corrigido substituindo o argumento da Eq. (2.24) por $z-\ell+d$. Esta correção, que eu obtive empiricamente resolvendo o modelo termodinâmico e comparando com os perfis do modelo contínuo, não altera os resultados significativamente pois o comprimento característico $\ell$ nas DMHs estudadas nesta tese são da ordem da largura $d$ de uma monocamada.
} 


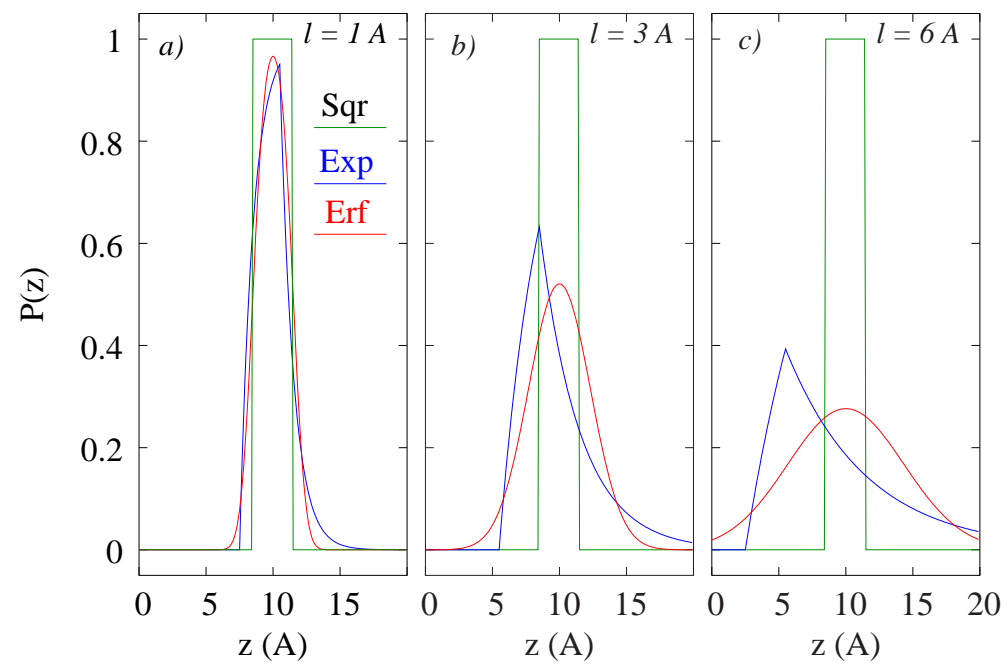

Figura 2.7: Perfis que concentração que simulam o efeito de diluição dos íons magnéticos devido a segregação e interdifusão. Os painéis mostram as funções de perfil quadrado (sqr), exponencial (exp) e de função-erro (erf) para três comprimentos característicos distintos. A largura da barreira é $d=3 \AA$.

contínua, similar à Eq. (2.24), para o caso de interdifusão

$$
\wp_{\mathrm{erf}}(z)=\frac{1}{2} \sum_{j=1}^{N_{b}}\left[\operatorname{erf}\left(\frac{z-\left(Z_{j}-d / 2\right)}{\ell}\right)-\operatorname{erf}\left(\frac{z-\left(Z_{j}+\frac{d}{2}\right)}{\ell}\right)\right] .
$$

Aqui, o comprimento característico $\ell$ é relacionado com a constante de difusão $D$ e a duração $t$ do processo de interdifusão por $\ell=2 \sqrt{D t}$.

Perfil ideal (quadrado) e o caso DMS O perfil ideal usado na Ref. [39] para descrever monocamadas perfeitas de MnSe é o perfil quadrado apresentado no Capítulo 1, Eq. (1.8), a saber

$$
\wp_{\mathrm{sqr}}(z)=\sum_{j=1}^{N_{b}} \Theta\left(z-Z_{j}+\frac{d}{2}\right) \Theta\left(\frac{d}{2}+Z_{j}-z\right) .
$$

Note que ambos $\wp_{\operatorname{erf}}(z)$ e $\wp_{\exp }(z)$ reproduzem o perfil ideal da Eq. (2.26) no limite de interdifusão e segregação desprezíveis, $\ell \rightarrow 0 .^{7}$

Para descrever o caso limite (bulk) de uma estrutura do tipo DMS, em que as impurezas magnéticas são uniformemente distribuídas na região do poço, basta considerar cada monocamada dentro do poço como uma barreira magnética e descrevê-las usando um dos modelos de perfil aqui definidos. O perfil resultante será essencialmente independente da escolha do modelo de perfil,

\footnotetext{
${ }^{7}$ De fato, para o caso de segregação e quando a Eq. (2.24) está com a correção indicada na nota de rodapé 6 , quando $\ell=0$ o perfil é quadrado, mas uma monocamada deslocada no sentido contrário ao crescimento. Isto ocorre pois $\ell=0$ corresponde segregação completa $\left(E_{s}=\infty\right)$.
} 
exceto por diferenças nas extremidades do poço (menores para pequenos $\ell$ 's) - o impacto destas diferenças na estrutura eletrônica é mínimo.

A Figura 2.7 compara os três perfis apresentados aqui para o caso de uma monocamada. Nos capítulos seguintes eu analiso uma série de DMHs que foram experimentalmente investigadas. Os resultados são tais que, o comprimento característico que melhor reproduziu os dados experimentais é $\ell$ da ordem da largura da monocamada $(d)$, mais precisamente $d \lesssim \ell \lesssim 2 d$. Os painéis (b) e (c) da Fig. 2.7 ilustram os casos de $\ell=d$ e $\ell=2 d$, respectivamente.

\subsection{Perfis de concentração: nominal e efetiva}

Usando os perfis modelos das Eqs. (2.24), (2.25) e (2.26) definidos na seção anterior, agora eu vou escrever as funções para as concentrações de impurezas magnéticas. Dois casos são considerados: o primeiro é a concentração nominal $x(z) \mathrm{e}$ o segundo é a concentração efetiva $\bar{x}(z)$.

\subsubsection{Perfil de concentração nominal}

Os perfis $\wp(z)$ definidos nos parágrafos anteriores são funções unitárias; eles são normalizados de forma a satisfazer

$$
\frac{1}{N_{b} d} \int_{L} \wp(z) \mathrm{d} z=1
$$

sendo $L$ o comprimento da amostra. Assim, posso definir um perfil de concentração contínuo $x(z)$, que leva em conta os efeitos de diluição, atribuindo uma concentração $x_{p}$ para as camadas descritas pela função $\wp(z)$, ou seja,

$$
x(z)=x_{p \wp}(z)
$$

Este perfil nominal não considera aglomeração antiferromagnética. Ele é usado para calcular, por exemplo, os offsets na estrutura de bandas devido a presença de Mn como impureza substitucional (Sec. 1.4.4).

\subsubsection{Perfil de concentração efetiva}

Como indicado na Sec. 2.2, a aglomeração antiferromagnética das impurezas de Mn é fundamental na determinação do desdobramento de spin induzido por troca em DMHs ideais, mesmo que apenas qualitativamente [39]. Egues e Wilkins [39] consideraram aglomerados Mn-Mn usando uma concentração planar efetiva $\bar{x}_{p}=x_{p}\left(1-x_{p}\right)^{4}$, com $x_{p}$ a fração molar de Mn em uma monocamada. Esta 


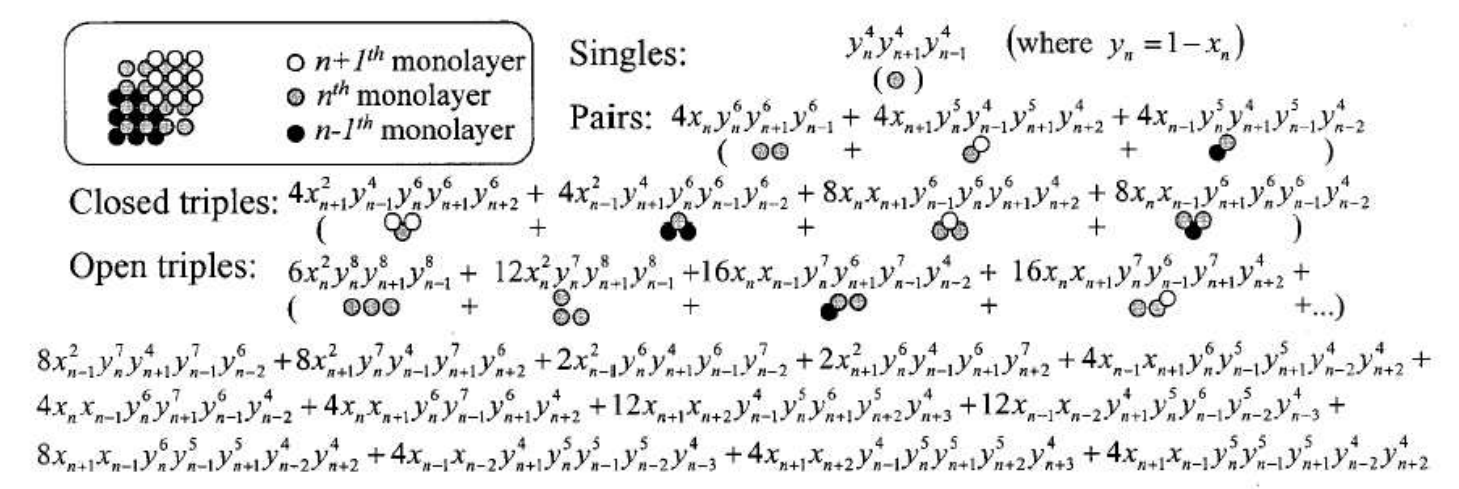

Figura 2.8: Probabilidades de um spin na monocamada $n$ participar de um aglomerado dada uma distribuição arbitrária de íons de Mn. Multiplicando estas probabilidades por $x_{n}$, obtém-se o número de spins que que estão no respectivo aglomerado. Os cátions nas monocamadas $n+1, n$ e $n-1$ estão indicados por branco, cinza e preto, respectivamente. Extraído de Crooker et al. [7].

concentração efetiva para uma monocamada ideal corresponde ao modelo de aglomeração $P_{1}$ da Tabela 2.1 .

Generalizar este conceito para perfis de concentração contínuos é direto. Um dado sítio de $\mathrm{Mn}$ possui vizinhos próximos que pertencem a três monocamadas adjacentes [59, 61], como ilustrado na Fig. 2.3. Esta característica precisa ser considerada explicitamente na definição da concentração efetiva de forma que os casos ideal e bulk sejam recuperados como situações limites. Para o caso mais simples de singletos, a probabilidade de encontrar uma única impureza numa distribuição arbitrária de Mn é igual a probabilidade dela não estar formando um par com seus vizinhos próximos, i.e.,

$$
P_{1}(z)=\left[1-x_{p} \wp(z-d)\right]^{4}\left[1-x_{p} \wp(z)\right]^{4}\left[1-x_{p} \wp(z+d)\right]^{4} .
$$

Aqui a função $\wp(z)$ é um perfil arbitrário qualquer, incluindo as funções perfis apresentadas nos parágrafos anteriores. $P_{1}(z)$ é uma generalização da probabilidade $P_{1}$ da Tabela 2.1. Assim, para este caso, eu defino um perfil de concentração efetiva

$$
\bar{x}(z)=x_{p} \wp(z) P_{1}(z) .
$$

Para os casos incluindo tripletos e aglomerados maiores, é possível obter um perfil de concentração efetiva similar à Eq. (2.30) substituindo a probabilidade $P_{i}$ em (2.4) e (2.5), respectivamente, por suas generalizações contínuas, como a Eq. (2.29), e multiplicando estas equações por $x_{p} \wp(z)$. Expressões explícitas para $P_{i}(z)$ para aglomerados maiores podem ser encontradas na Ref. [7], aqui reproduzidas na Fig. 2.8 .

A Fig. 2.9 compara perfis de concentração efetivos $\bar{x}(z)$ para os casos 


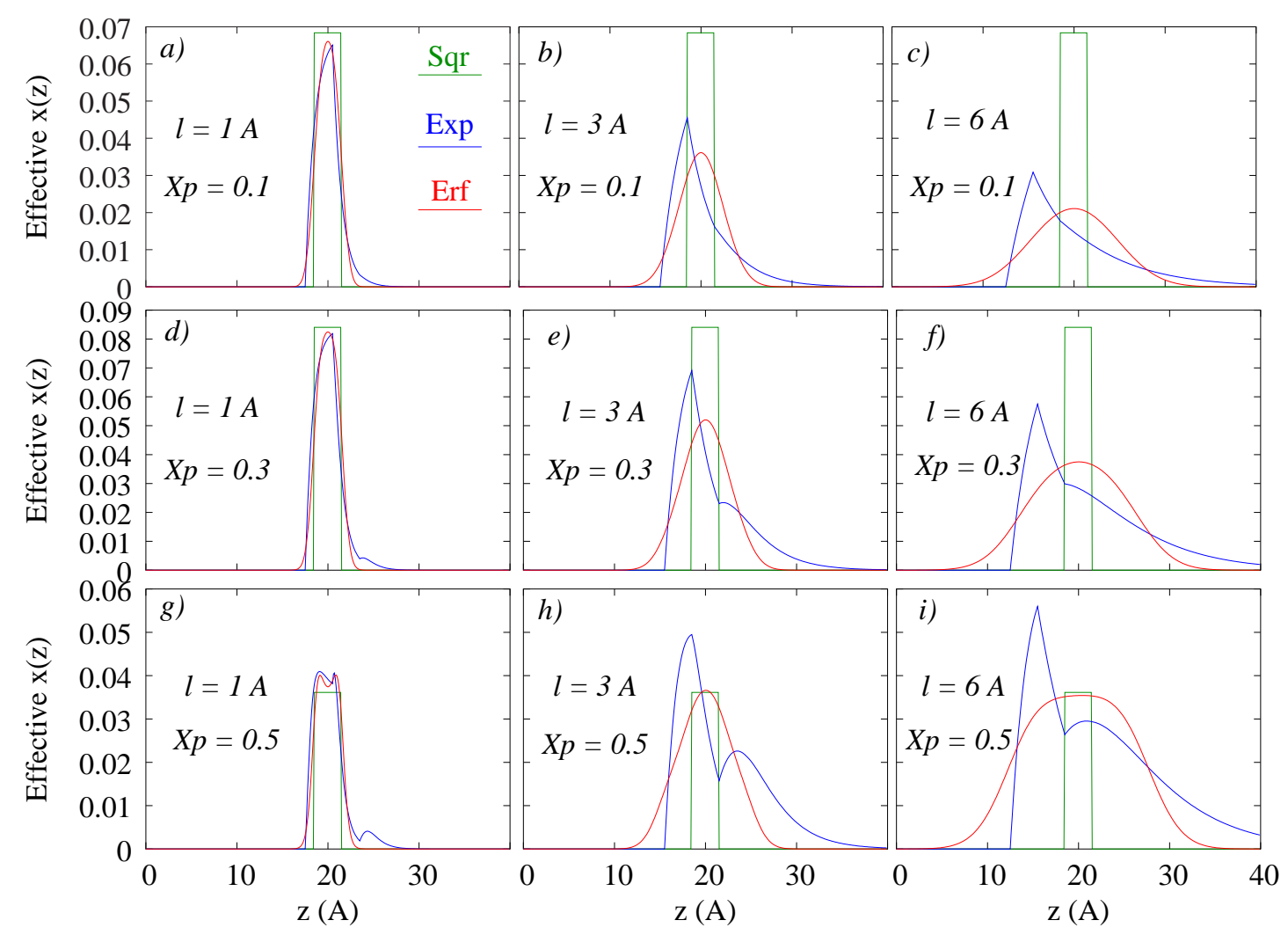

Figura 2.9: Perfis de concentração efetivos $\bar{x}(z)$ para diversos tipos de perfil, comprimentos característicos $\ell$ e concentrações nominais $x_{p}$. O modelo de aglomerados usado foi $P_{1}+P_{3}$ e a largura das barreiras é $d=3 \AA$. As oscilações no perfil ocorrem pois a concentração efetiva diminui (aumenta) quando a concentração nas camadas adjacentes é maior (menor).

ideal, segregação e interdifusão para várias comprimentos característicos $\ell$. Ao comparar com os perfis nominais da Fig. 2.7, nota-se que (i) a magnitude da concentração efetiva é significativamente menor devido à aglomeração, e (ii) a formação de pares antiferromagnéticos entre camadas adjacentes introduz modulações em $\bar{x}(z)$ ausentes no perfil nominal $x(z)$.

Concentração média $\langle\bar{x}\rangle$ A título de comparação entre diferentes perfis é interessante definir uma concentração efetiva média $[67,78]$

$$
\langle\bar{x}\rangle=\frac{1}{N_{b} d} \int_{L} \bar{x}(z) \mathrm{d} z
$$

A Fig. 2.10 mostra $\langle\bar{x}\rangle$ para o caso de uma única monocamada cujo conteúdo magnético é diluído por segregação (para interdifusão os resultados são qualitativamente similares). Além do aumento do spin efetivo, e consequentemente da concentração efetiva, o efeito extrínseco também diminui as diferenças entre os modelos de aglomerados.

Para estes perfis de concentração efetiva, $\langle\bar{x}\rangle\left\langle x_{p}\right.$ sempre, devido 

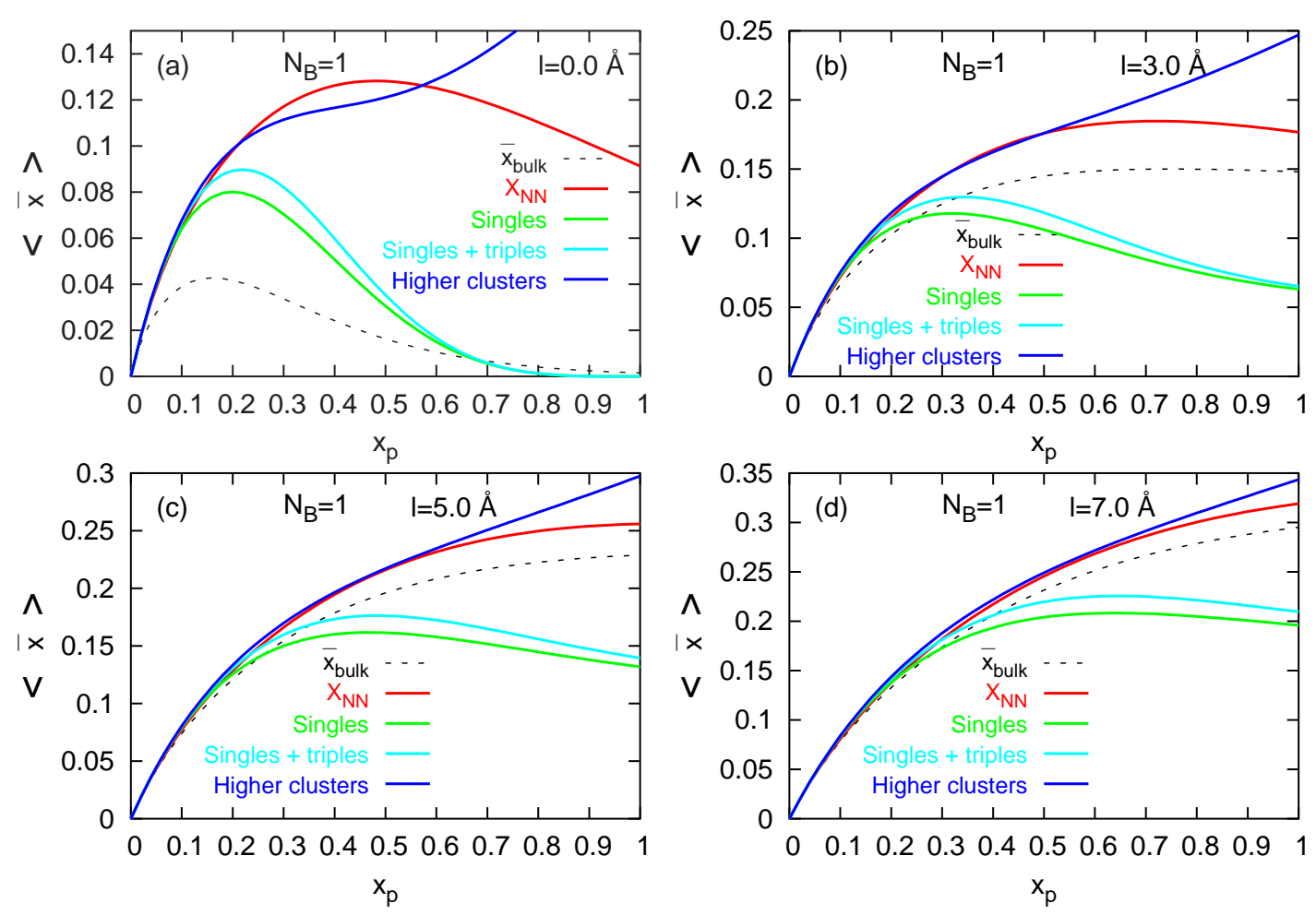

Figura 2.10: Efeito da diluição sobre a concentração efetiva média $\langle\bar{x}\rangle$. O perfil de concentração usado foi de segregação com comprimento característico $\ell$. Para $\ell=0.0 \mathrm{~nm}$, (a) reproduz o resultado analítico da Fig. 2.4(c). Com o aumento da diluição ( $\ell$ maior), (i) a concentração efetiva média aumenta, e (ii) as diferenças entre os modelos de aglomeração diminui.

a aglomeração antiferromagnética. Note que a Eq. (2.31), usando o perfil da Eq. (2.30) e suas contrapartidas para tripletos e aglomerados maiores, reduz-se a $\bar{x}_{p}$ quando $\wp(z)=\wp_{\text {sqr }}(z)$, e reduz-se a $\bar{x}$ quando $\wp(z)=\wp_{\text {DMS }}(z)$. Ou seja, as funções aqui definidas recuperam os casos limites.

A Eq. (2.31), ao integrar sobre todo o perfil, considera a sobreposição entre barreiras adjacentes. Como em geral nestas DMHs a região magnética é dentro da região de confinamento, a função de onda eletrônica se extende sobre várias barreiras magnéticas. Isto faz que a concentração efetiva do conjunto seja a grandeza relevante. Isto está ilustrado na Fig. 2.11, mostrando que para perfis não-ideais, a concentração efetiva média depende fortemente não apenas de $x_{p}$ [cf. Eq. (2.30) e as definições para as funções $\wp(z)$ ], mas também do número $N_{b}$ de barreiras magnéticas dentro do poço. Maior o número destas barreiras, menor as separações entre elas e, consequentemente, maior a chance de átomos pertencentes a barreiras adjacentes formarem pares antiferromagnéticos. Note que as diferenças entre os perfis exponencial e função-erro são relativamente pequenas, mas aumentam com $x_{p}$.

A concentração média é uma grandeza que pode ser usada para pla- 


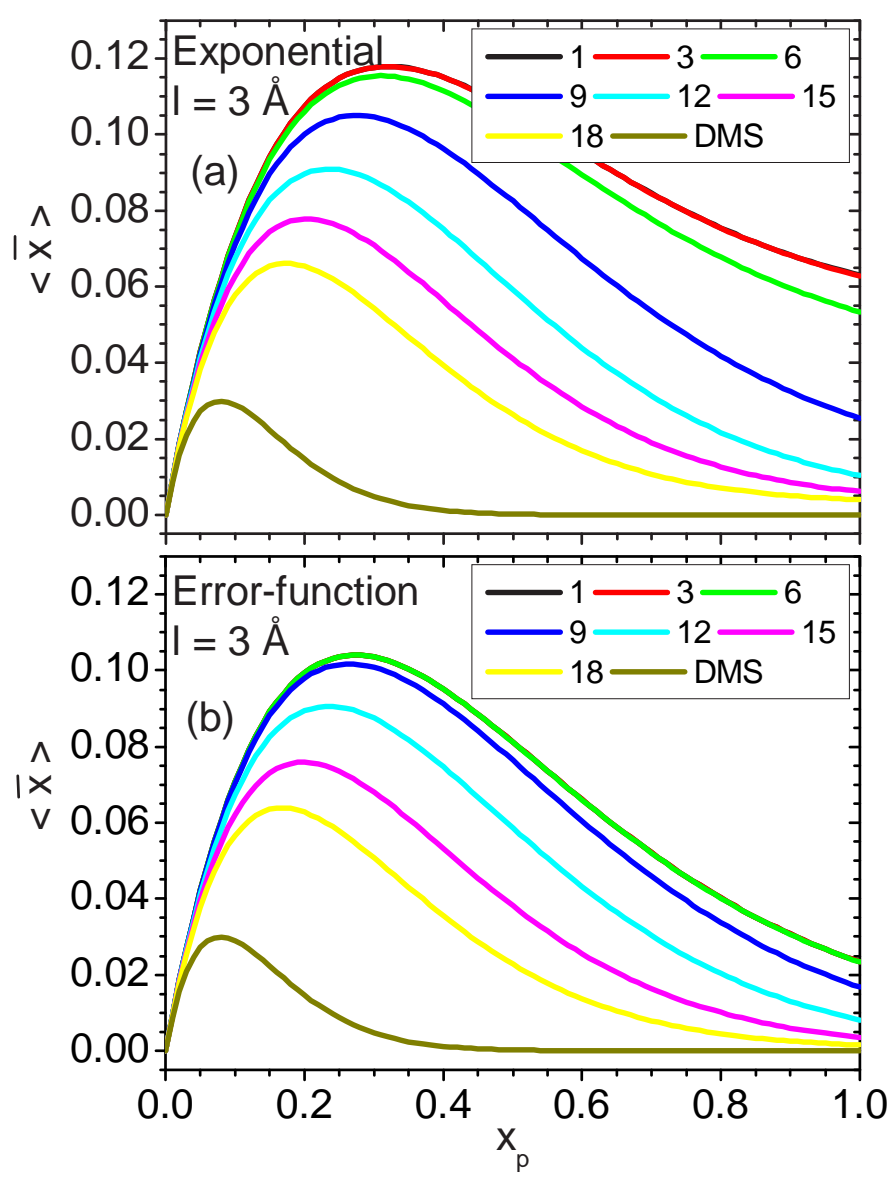

Figura 2.11: Concentração efetiva média $\langle\bar{x}\rangle$ em função da concentração planar $x_{p}$, para os perfis exponencial (a) e de função erro (b). A concentração total de Mn é $3 \mathrm{ML}$, distribuída num número diferente de barreiras. Cada curva corresponde à configuração $3 \times 1 / N_{b} \mathrm{ML}$, com $N_{b}$ indicado na legenda. O comprimento característico é $\ell=3 \AA$ nos dois casos. As $N_{b}$ barreira estão regularmente distribuídas em um poço quântico de largura $L=105 \AA$. O aumento do número de barreiras aumenta a sobreposição dos perfis de concentração e, consequentemente, diminui a concentração efetiva. Nota-se que o efeito do emparelhamento antiferromagnético é mais efetivo para o caso do perfil de interdifusão (b), $\langle\bar{x}\rangle$ menor que em (a). Em (a), curvas com $N_{b}=1$ e 3 estão sobrepostas; em (b), o mesmo ocorre para $N_{b}=1,3$ e 6 .

nejar a preparação de uma amostra. Por exemplo, para minimizar o emparelhamento anti-ferromagnético e, consequentemente, maximizar a interação de troca $s$-d, basta utilizar o valor de $x_{p}$ que corresponde ao máximo da curva de $\bar{x}$ para um dado número de barreiras (Fig. 2.11).

Note que o comprimento característico também deve ser levado em consideração neste tipo de análise de concentração média. A Fig. 2.12 é um diagrama de curvas de nível da concentração efetiva média $\langle\bar{x}\rangle$ em função de $\ell$ e $x_{p}$. Para concentrações nominais pequenas, $\langle\bar{x}\rangle$ depende fracamente do comprimento 


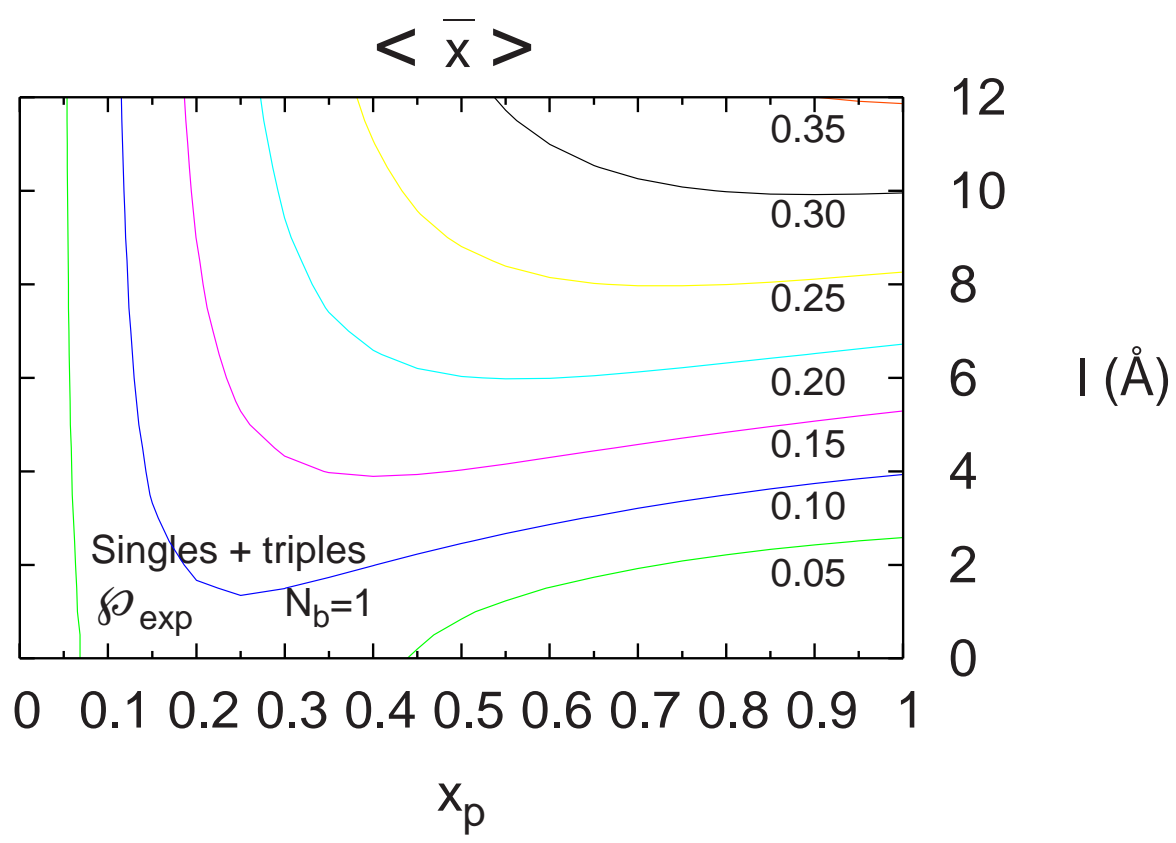

Figura 2.12: Curvas de nível da concentração efetiva média $\langle\bar{x}\rangle$ em função concentração planar $x_{p}$ e do comprimento característico $\ell$ dos perfis. Várias combinações de $x_{p}$ e $\ell$ podem ser usadas para obter a mesma concentração efetiva média. Para baixos (altos) valores de $x_{p}$, a concentração média não depende (depende) da diluição. Curvas similares também são obtidas para o perfil de interdifusão e para os outros modelos de aglomerados.

característico. Isto porque a amostra já é muito diluída tal que $\bar{x}(z) \approx x(z)$, implicando em $\langle\bar{x}\rangle \approx x_{p}$. Já para concentrações elevadas a concentração efetiva depende sim fortemente com $\ell$ uma vez que a diluição passa a ser essencial.

Concentração efetiva $x_{e f f}$ No que se refere a interação de troca $s p$-d, o importante é a sobreposição das funções de onda eletrônicas com os íons de Mn. Neste sentido, aqui eu defino uma outra concentração efetiva que leva em consideração esta sobreposição, a saber

$$
x_{e f f}=\int_{L} \bar{x}(z)|\chi(z)|^{2} \mathrm{~d} z
$$

Uma aplicação prática desta grandeza é fazer uma estimativa da contribuição de troca $s p-d$ em primeira ordem de teoria de perturbação. Neste caso, usando a definição $(2.32)$ e sendo $\chi^{0}(z)$ a função de onda não perturbada, tenho

$$
E^{(1)}=\int_{L}-\frac{1}{2} \bar{x}(z) N_{0} \alpha\left\langle S_{z}\right\rangle \sigma_{z}\left|\chi^{0}(z)\right|^{2} \mathrm{~d} z \equiv-\frac{1}{2} x_{e f f} N_{0} \alpha\left\langle S_{z}\right\rangle \sigma_{z}
$$

Nesta aproximação, defino o desdobramento de spin devido a interação de troca 

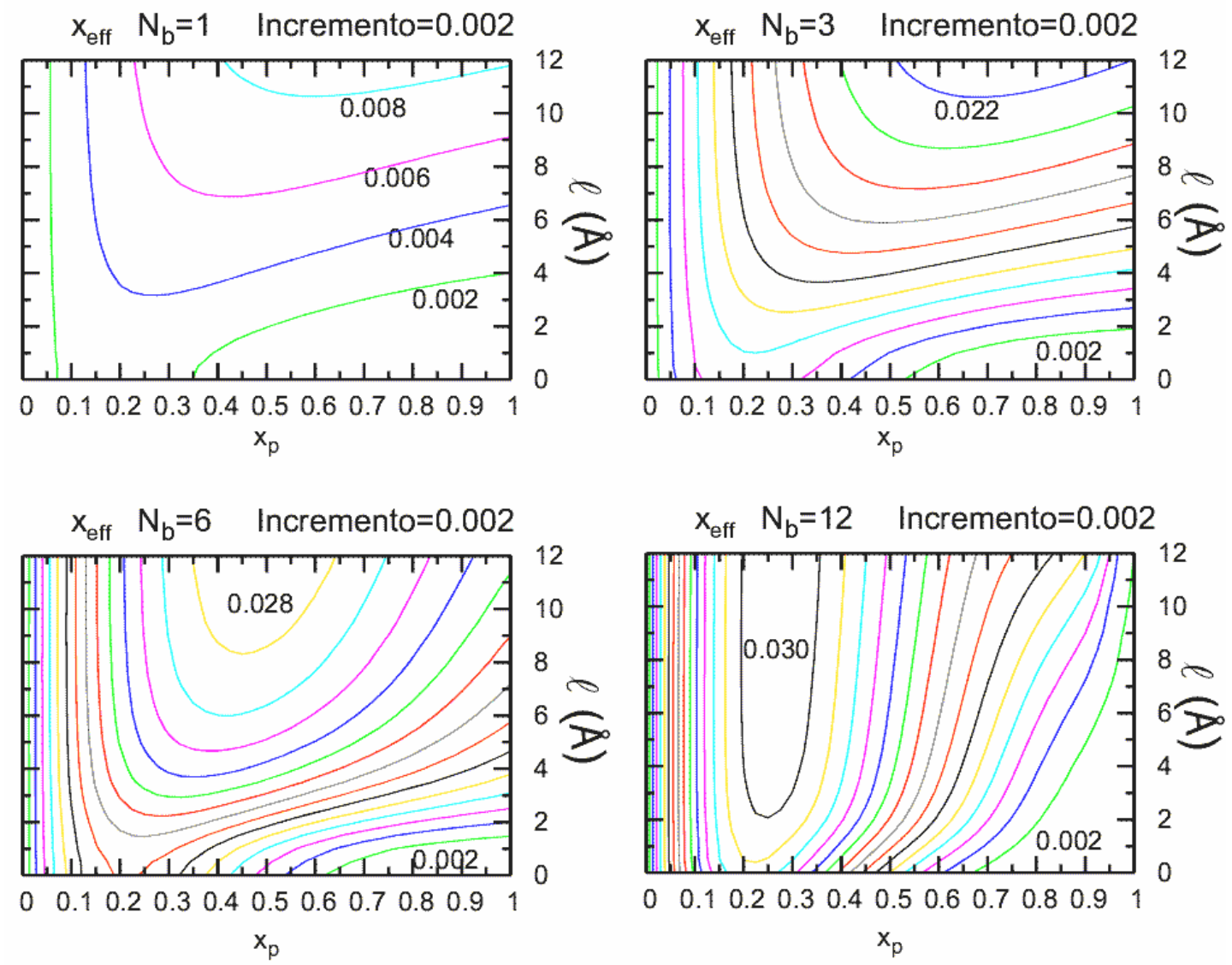

Figura 2.13: Curvas de nível da concentração efetiva $x_{\text {eff }}$ em função da concentração planar $x_{p}$ de $M n$ e do comprimento característico $\ell$. Estas curvas são para o perfil de concentração exponencial (segregação) e cada painel é para um diferente número $N_{b}$ de barreiras magnéticas. À medida que $N_{b}$ aumenta, a as regiões em que $x_{e f f}$ é máximo ocorrem para $x_{p}$ e $\ell$ menores. O incremento de $x_{\text {eff }}$ a cada curva de nível é 0.002 .

s-d em termo de $x_{e f f}$,

$$
\Delta E \equiv E_{s-d}^{\uparrow}-E_{s-d}^{\downarrow} \equiv-x_{e f f} N_{0} \alpha\left\langle S_{z}\right\rangle
$$

Esta definição será particularmente útil nos Caps. 5 e 6 .

A Figura 2.13 mostra curvas de nível de $x_{\text {eff }}$ em função da concentração nominal $x_{p}$ e do comprimento característico $\ell$, para o caso de perfil de segregação (gráficos similares são obtidos para interdifusão). As curvas foram obtidas usando o hamiltoniano e os parâmetros das simulações do próximo capítulo, Tabela 3.1 na pág. 49.

Uma inspeção da Fig. 2.13 fornece uma ideia de como não é trivial caracterizar o perfil de concentração de impurezas magnéticas presente em uma amostra. Por exemplo, seja uma medida de magnetofotoluminescência que fornece um valor para $\Delta E$ e, via a Eq. (2.34), um valor correspondente para $x_{e f f}$. 
Analisando as curvas de nível, percebe-se que existem diversas escolhas para a concentração nominal $x_{p}$ e comprimento característico $\ell$ que resultam no mesmo $x_{e f f}$. Adicionalmente, existem curvas similares àquelas da Fig. 2.13 para o caso de interdifusão, e que também fornecem o memo valor para $x_{e f f}$.

As curvas de nível também mostram que, para baixas concentrações nominais $x_{p}$, a concentração efetiva é pouco sensível ao comprimento característico do perfil, o contrário ocorrendo para altas concentrações. À medida que $N_{b}$ aumenta, a curva de nível da concentração efetiva máxima desloca-se para $x_{p}$ e $\ell$ menores. Isto ocorre pois a sobreposição entre os perfis de barreiras vizinhas aumenta com $N_{b}$, o que acentua a aglomeração antiferromagnética e, portanto, diminui $x_{e f f}$.

\subsection{Discussões}

Uma vez que vários dos gráficos aqui apresentados variam a concentração nominal até a unidade, é importante lembrar os limites de validade dos modelos de aglomeração. A hipótese básica da estatística de aglomerados é a distribuição aleatória das impurezas magnéticas. Para baixas concentrações é natural que ela seja satisfeita. Já para altas concentrações de Mn não se espera que isto continue ocorrendo. De fato, acima do limite de percolação $x_{c}$, existe uma probabilidade não nula de que cada impureza pertença a um aglomerado infinito. Para bulk $(\mathrm{Cd}, \mathrm{Mn}) \mathrm{Te}$, com uma rede fcc tridimensional, $x_{c} \approx 0.19$, enquanto para uma monocamada de $(\mathrm{Cd}, \mathrm{Mn}) \mathrm{Te}$ com uma rede bidimensional quadrada $x_{c}=1 / 2$ [61]. Por outro lado, à medida que o tamanho do aglomerado aumenta, a magnetização por spin diminui, explicando porque resultados usando a estatística de aglomerados concordam com experimentos para altas concentrações, pelo menos para bulk [67].

Se todos os resultados experimentais aqui investigados fossem de amostras com pequenas concentrações de $\mathrm{Mn}$, eu estaria dentro do limite de validade dos modelos. Entretanto, várias amostras têm concentrações nominais altas, inclusive igual à unidade. Nestes casos é importante sempre lembrar que $\ell$ deve ser considerado com um parâmetro de ajuste do modelo, e a sua associação com o comprimento característico real de diluição deve ser feita com cautela.

O diagrama da Fig. 2.14 resume o papel de aglomeração e de diluição em DMHs. Da esquerda para a direita mostro o caso bulk, com concentração tridimensional $x$, passando para o caso bidimensional ideal com concentração planar $x_{p}$, e depois o caso não ideal, com um perfil de concentração diluído ao longo da direção de crescimento, seja por segregação ou por interdifusão. Na direção vertical, mostro que a distribuição espacial dos átomos de Mn, i.e., concentração 


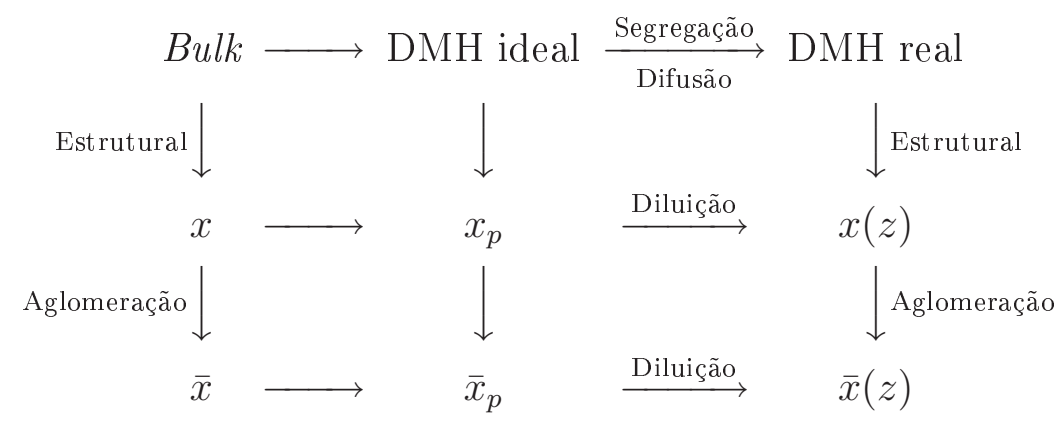

Figura 2.14: Esquema dos efeitos de aglomeração e diluição em DMHs.

estrutural, corresponde a uma concentração efetiva no que se refere a efeitos de magnetismo relacionado ao $\mathrm{Mn}$.

Os modelos que defini para os perfis de concentração nominal (2.28) e efetiva (2.30) permitem tratar os efeitos de diluição e segregação/diluição, respectivamente, de uma maneira bastante simples. Usando estes perfis de concentração para substituir os casos ideais apresentados na Seção 1.4.4, eu analiso no próximo capítulo alguns resultados experimentais para desdobramento de spin e tempos de espalhamento de spin, ambos induzidos pela interação de troca $s p-d$. 


\section{Capítulo 3}

\section{Desdobramento de Spin e Tempo de Espalhamento}

\section{Conteúdo}

Neste capítulo eu calculo do desdobramento de spin $\Delta E$ de transições elétron-buraco-pesado, e os tempos de espalhamento de spin $\tau_{s f}$ de elétrons na banda de condução, ambos induzidos pela interação de troca $s p-d$. Aqui eu utilizo os perfis de concentração do Cap. 2 para levar em consideração os efeitos de aglomeração antiferromagnética e de diluição dos íons de $\mathrm{Mn}$. Os resultados apresentados para $\Delta E$ e $\tau_{s f}$ descrevem satisfatoriamente os resultados experimentais disponíveis $[4,7]$ e complementam o trabalho de Egues e Wilkins [39]. Os modelos de aglomerados com singletos e tripletos retornaram melhores ajustes com os dados experimentais. Quanto à diluição, os comprimentos característicos foram da ordem de (1 a 2) ML. Entretanto, os modelos de segregação e interdifusão retornaram valores próximos e não foi possível distinguir qual dois dois mecanismos é predominante.

3.1 Introdução . . . . . . . . . . . . . . . . . . . . 46

3.2 Sistema modelo e abordagem teórica . . . . . . . . . . . . . . 48

3.3 Resultados: desdobramento de spin . . . . . . . . . . . . . 51

3.4 Resultados: tempo de espalhamento de spin . . . . . . . . . . . 59

3.5 Discussões ........................... 67 


\subsection{Introdução}

Espectroscopia magnetoóptica estática - fotoluminescência, absorção, e rotação de Faraday - é bastante usada para investigar a estrutura eletrônica em DMHs [51, 79]. Neste capítulo eu investigo as propriedades magnetoópticas de heteroestruturas digitais magnéticas (DMHs), com o enfoque nos efeitos do magnetismo das impurezas magnéticas, mais especificamente, da aglomeração antiferromagnética e da diluição dos íons de Mn. As estruturas em questão são poços quânticos ZnSe/(Zn,Cd)Se, crescidos na direção (100), com arranjos planares periódicos de impurezas magnéticas ( $M n, S=5 / 2$ ), Fig. 3.1. Aqui eu utilizo os perfis modelos introduzidos no Cap. 2 para descrever o potencial efetivo da interação de troca sp-d. Eu então calculo a estrutura eletrônica dependente de spin para elétrons (e) e buracos-pesados $(h h)$ no contexto teórico apresentado na Seção 1.4.4. Em seguida obtenho o desdobramento de spin gigante $\Delta E$ de transições $e-h h$, e os tempos de espalhamento de spin $\tau_{s f}$ de elétrons na banda de condução, ambos induzidos pela interação de troca $s p-d$. Eu mostro que a inclusão de aglomeração e diluição das impurezas magnéticas aproxima os resultados dos modelos para $\Delta E$ e $\tau_{s f}$ dos resultados experimentais disponíveis. A investigação aqui apresentada complementa o trabalho de Egues e Wilkins [39], que estudaram o efeito de aglomeração (modelo com singletos) mas sem incluir diluição (perfil ideal quadrado).

Esta parte do trabalho está direcionada para dois conjuntos de dados
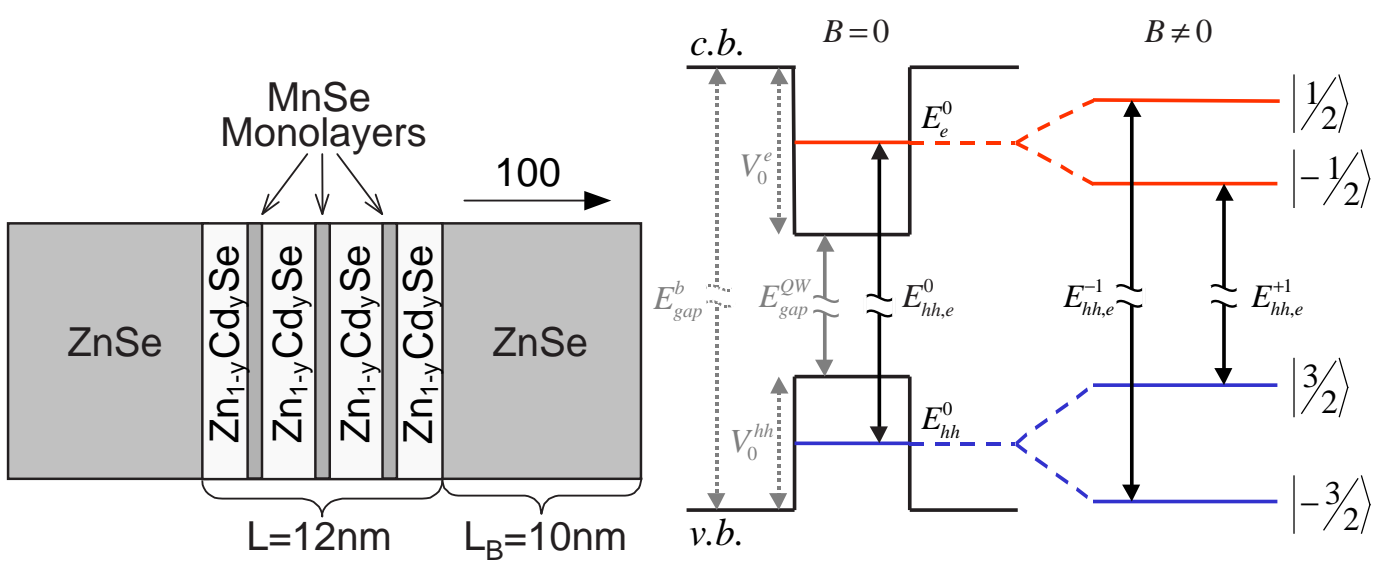

Figura 3.1: (Esquerda) Diagrama de um poço quântico digital magnético ideal, crescido na direção (100), e com monocamadas magnéticas de MnSe. (Direita) Esquema ilustrando a interação de troca $s p$ - $d$ entre os portadores e os momentos magnéticos de Mn localizados na região do poço. Esta interação é responsável pelo desdobramento de spin nas sub-bandas de elétrons $(e)$ e buracos-pesados $(h h)$ quando um campo magnético é aplicado. As transições dos pares $e-h h$ a campo zero $E_{e, h h}^{0}$ e com campo magnético aplicado $E_{e, h h}^{ \pm 1}$ são ilustradas. Estas transições opticamente ativas têm polarizações +1 e -1 . 


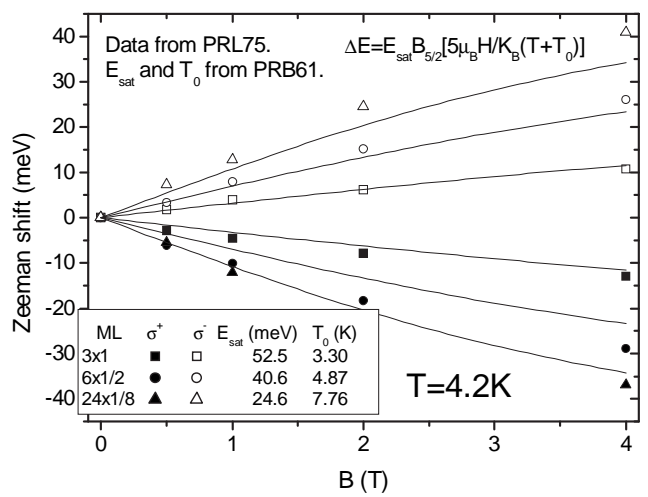

Figura 3.2: Desdobramento de spin gigante (símbolos) a $T=4.2 \mathrm{~K}$ para três amostras com diferentes números de barreiras e concentrações nominais [RI]. As linhas são funções de Brillouin usando os parâmetros obtidos em RII (indicados na legenda) para as mesmas amostras a $T=0.35 \mathrm{~K}$. No caso da amostra $24 \times 1 / 8 \mathrm{ML}$ foram usados os parâmetros da amostra $12 \times 1 / 4 \mathrm{ML}$ da Ref. [RII]. Baseado na Ref. [RI].

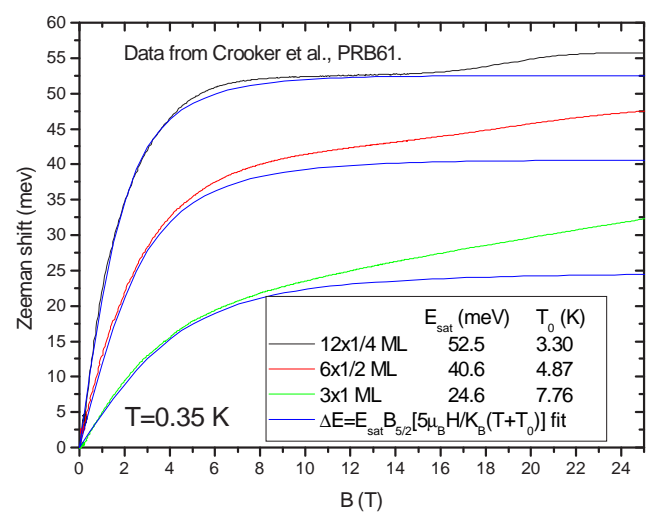

Figura 3.3: Desdobramento de spin gigante a $T=0.35 \mathrm{~K}$ para as configurações $3 \times 1 \mathrm{ML}$ (verde), $6 \times 1 / 2 \mathrm{ML}$ (vermelho), e $12 \times 1 / 4 \mathrm{ML}$ (preto) [RII]. As curvas azuis são ajustes da função de Brillouin para o dados a baixo campo (parâmetros indicados na legenda). Para campos $B \gtrsim 4 \mathrm{~T}$ os dados experimentais começam a exibir $d e-$ graus de magnetização. Baseado na Ref. [RII].

experimentais de S. A. Crooker e colaboradores [4, 7]. Os resultados das duas referências são para o mesmo conjunto de amostras, mas para condições experimentais diferentes. No caso da Ref. [4], que me refiro neste capítulo como RI, a temperatura das medidas foi de $T=4.2 \mathrm{~K}$ e os valores de campo magnético para as medidas de $\Delta E$ foram até $B=4 \mathrm{~T}$ em intervalos de $0.5 \mathrm{~T}$ (Fig. 3.2). Nestes experimentos, observou-se que o desdobramento de spin aumenta à medida que uma dada concentração de Mn é particionada em um número maior de planos. RI também contém os dados para $\tau_{s f}$ que eu analiso (Fig. 3.10 na pág. 59).

Já os dados da Ref. [7], a qual vou me referir como RII, foram obtidos a $T=0.35 \mathrm{~K}$ para $B$ até $60 \mathrm{~T}$ em intervalos de $0.15 \mathrm{~T}$ [80]. O trabalho em RII investiga justamente a questão de aglomeração de Mn. Na faixa de campo magnético investigada, os autores observam degraus de magnetização; aqui eu analiso apenas os resultados a "baixo" campo $(B \leqslant 4.0 \mathrm{~T})$, antes do aparecimento do primeiro degrau (e.g., veja na Fig. 3.3 o aparecimento de degraus de magnetização, mais evidente no caso $12 \times 1 / 4 \mathrm{ML}){ }^{1}$

\footnotetext{
${ }^{1}$ A notação $N_{b} \times x_{p}$ ML indica $N_{b}$ camadas magnéticas, cada uma com concentração nominal de Mn igual $x_{p}$ monocamadas (ML - monolayer).
} 
Escopo da abordagem Aqui eu utilizo os resultados do Cap. 2, no qual defino perfis modelos apropriados para a concentração de impurezas magnéticas e, adicionalmente, perfis de concentração efetiva. Estes perfis são funções "contínuas" que interpolam entre o caso bulk e o estritamente bidimensional. Isto permite considerar a aglomeração de Mn em todos os perfis modelos de uma forma bastante direta. O resultado desta abordagem é, com a introdução de perfis nãoideais e as correspondentes concentrações efetivas de Mn, melhorar os resultados dos cálculos anteriores [39]. Em particular, $(i)$ o desdobramento de spin $\Delta E$ descreve melhor os dados experimentais disponíveis [RI, RII]; (ii) os tempos de espalhamento de spin $\tau_{s f}$ induzidos por troca $s-d$ a campo magnético nulo ficam numa faixa mais reduzida de valores - como nos resultados experimentais - para diferentes configurações com Mn (para $B \neq 0$ os resultados para $\tau_{s f}$ não alteram sensivelmente, quando comparado com [39]); e (iii) obtenho valores não nulos para $\Delta E$ e $1 / \tau_{s f}$, inclusive para o caso limite de concentração nominal de $\mathrm{Mn}$ igual à unidade, $x_{p}=1 .^{2}$

Organização do capítulo Na Sec. 3.2 eu apresento o sistema modelo e a formulação teórica do problema. Nas Secs. 3.3 e 3.4 eu apresento os resultados para os desdobramentos de spin e para os tempos de espalhamento de spin, respectivamente.

\subsection{Sistema modelo e abordagem teórica}

Os resultados experimentais nos quais eu baseio a minha análise teórica são de RI e RII. As DMHs em questão são poços quânticos de ZnSe/(Zn,Cd)Se com monocamadas de MnSe digitalmente incorporadas e igualmente espaçadas, como ilustrado na Fig. 3.1(a). As amostras diferem pelo número $N_{b}$ de barreiras magnéticas e pela concentração nominal $x_{p}$ das monocamadas. A concentração total de Mn é mantida constante em $3 \mathrm{ML}$, igualmente distribuída entre as barreiras, i.e., $x_{p}=3 / N_{b}$. As configurações são denotadas por " $N_{b} \times x_{p}$ ML", e.g., $3 \times 1 \mathrm{ML}$, $6 \times 1 / 2 \mathrm{ML}, 9 \times 1 / 3 \mathrm{ML}$ e assim por diante [RI].

Para calcular a estrutura eletrônica destes sistemas eu resolvo o hamiltoniano do sistema no contexto da teoria da massa efetiva (Cap. 1, Sec. 1.4.4). Aqui eu desconsidero a quantização de Landau e suponho bandas parabólicas (aproximação comum quando se estuda as propriedades magnetoópticas em DMSs de gap elevado [51, pág. 288]). ${ }^{3}$ Eu também faço uma simplificação adicional que

\footnotetext{
${ }^{2}$ Para $x_{p}=1$ e perfis ideais quadrados, como em [39], a concentração efetiva $\bar{x}_{p}=x_{p}(1-$ $\left.x_{p}\right)^{4}=0$ e, consequentemente, $\Delta E=0$ e $1 / \tau_{s f}=0$.

${ }^{3}$ Nos capítulos subsequentes, nos quais eu investigo magnetotransporte, a quantização de Landau é um ingrediente essencial e, portanto, é apropriadamente considerada na formulação.
} 
Tabela 3.1: Parâmetros utilizados no modelo para simular o poço quântico digital magnético investigado experimentalmente em RI e RII. Eu sigo as escolhas de parâmetros da Ref. [39] para comparar aqueles resultados com os calculados nesta tese.

\begin{tabular}{|c|c|}
\hline Parâmetros & Buraco pesado $(\Lambda=h h)$ \\
\hline Massa efetiva & $m_{e} / m_{0}=0.16$ \\
\hline Offset $\mathrm{ZnSe} / \mathrm{Zn}_{0.8} \mathrm{Cd}_{0.2} \mathrm{Se}^{\dagger}$ & $v_{0}^{e}=185 \mathrm{meV}$ \\
\hline Offset $\mathrm{Zn}_{0.8} \mathrm{Cd}_{0.2} \mathrm{Se} / \mathrm{MnSe}^{\dagger}$ & $v_{b}^{h h}=100 \mathrm{meV}$ \\
\hline Interação de troca $s p-d$ & $N_{0} \beta=-1.1 \mathrm{eV}$ \\
\hline Largura do poço & $L=120 \AA$ \\
\hline Largura barreiras laterais & $L_{B}=100 \AA$ \\
\hline Largura barreiras magnéticas & $d=3 \AA \ddagger$ \\
\hline Número de barreiras com Mn & $N_{b}=1,3,6 \ldots, 24$ \\
\hline Concentração planar de Mn & $x_{p}=3 / N_{b}^{\ddagger}$ \\
\hline Comprimento de diluição & $0 \lesssim \ell \lesssim 10 \AA$ \\
\hline Temperatura & $T=4.2 \mathrm{~K}[\mathrm{RI}]$ e $T=0.35 \mathrm{~K}[\mathrm{RII}]$ \\
\hline Temperatura efetiva & $3 \mathrm{~K} \lesssim T_{0} \lesssim 8 \mathrm{~K}$ \\
\hline
\end{tabular}

${ }^{\dagger}$ Veja os exemplos no Cap. 1, pág. 10.

${ }^{\ddagger}$ Para $N_{b}=1, x_{p}=1$ e $d=9 \AA$.

é ignorar a interação de Coulomb entre os portadores $(e-e, e-h h$ e $h h-h h)$. Neste contexto, as energias de sub-banda $\varepsilon_{i, \sigma_{z}}^{\Lambda}$ e as funções envelope $\chi_{i, \sigma_{z}}^{\Lambda}$ são então calculadas resolvendo a equação de Schrödinger unidimensional [Eq. (1.14), pág. 15]

$$
\left[-\frac{\hbar^{2}}{2 m_{\Lambda}^{*}} \frac{\partial^{2}}{\partial z^{2}}-v_{\sigma_{z}}^{\Lambda}(z)\right] \chi_{i, \sigma_{z}}^{\Lambda}(z)=\varepsilon_{i, \sigma_{z}}^{\Lambda} \chi_{i, \sigma_{z}}^{\Lambda}(z)
$$

para cada componente de spin $\left(\sigma_{z}^{\Lambda}= \pm 1\right)$ de elétrons $(\Lambda=e)$ e buracos pesados $(\Lambda=h h)$. O potencial

$$
v_{\sigma_{z}}^{\Lambda}(z)=v_{0}^{\Lambda}\left[1-\wp_{\mathrm{sqr}}(z ; 1,0, L)\right]+v_{b}^{\Lambda} x(z)+v_{\mathrm{sp}-\mathrm{d}}^{\Lambda, \sigma_{z}} \bar{x}(z)
$$

descreve os potenciais estruturais e magnéticos da DMH da Fig. 3.1; nele eu substituí o perfil de concentração ideal (quadrado) $x_{p} \wp_{\mathrm{sqr}}(z)$ das Eqs. (1.9), (1.10) e (1.11) pelos perfis de concentração nominal $x(z)$ e efetiva $\bar{x}(z)$ do Cap. 2, Eqs. (2.28) e (2.30), respectivamente. As magnitudes dos termos de troca sp$d$ são [Eq. (1.7)]

$$
\begin{aligned}
& v_{s p-d}^{e, \sigma_{z}}=-\frac{1}{2}\left\langle S_{z}\right\rangle N_{0} \alpha \sigma_{z}^{e}, \\
& v_{s p-d}^{h h, \sigma_{z}}=\frac{1}{2}\left\langle S_{z}\right\rangle N_{0} \beta \sigma_{z}^{h h} .
\end{aligned}
$$

para elétrons e buracos pesados, respectivamente. Os demais parâmetros são 


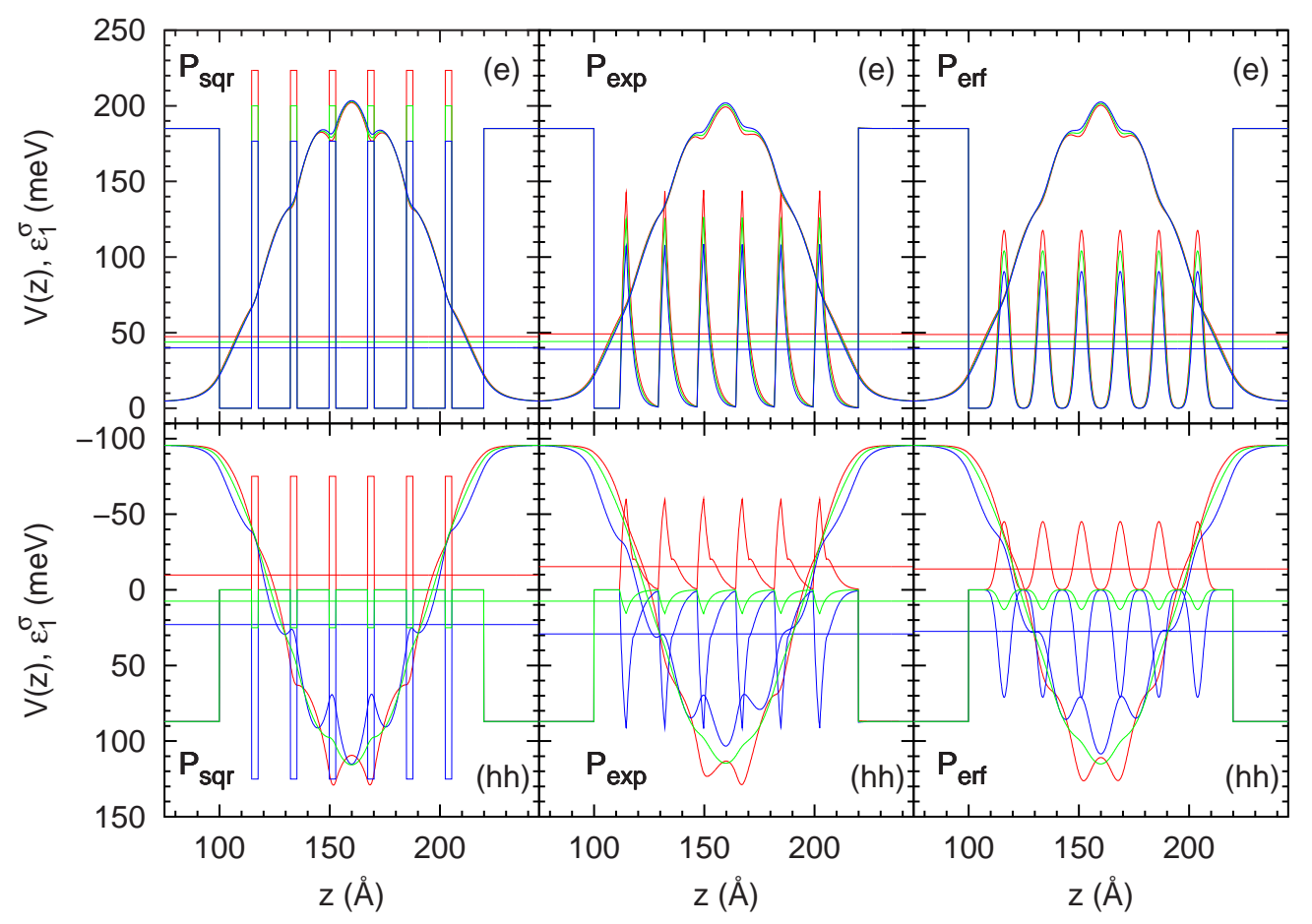

Figura 3.4: Ilustração dos perfis de potencial das bandas de condução (painéis superiores) e banda de valência (painéis inferiores) para os perfis ideal (sqr), de segregação (exp), e interdifusão (erf). Estão sobrepostos três casos: $B=0$ (verde), spin para cima (vermelho) e spin para baixo (azul) a $B=4 \mathrm{~T}$. As funções de onda e as energias de sub-banda para cada caso também são apresentadas. Note que a principal contribuição para o desdobramento de spin total vem dos buracos pesados, cuja constante de troca, $N_{0} \beta=-1.11 \mathrm{eV}$, é consideravelmente superior que a dos elétrons, $N_{0} \alpha=0.26 \mathrm{eV}$. Os parâmetros usados foram $x_{p}=$ $0.25, T+T_{0}=5 \mathrm{~K} \mathrm{e} \ell=3.0 \AA$.

listados na Tabela 3.1.

A Figura 3.4 ilustra os perfis de potencial para os casos ideal, segregação e interdifusão. Note a dependência de spin na região das barreiras magnéticas. A altura da barreira é modulada pela interação de troca $s p$-d, termo $v_{s p-d}^{\Lambda, \sigma_{z}}$ na Eq. (3.2). A altura das barreiras diminui como função do campo magnético para elétrons $|-1 / 2\rangle$ e buracos pesados $|3 / 2\rangle$ enquanto elas aumentam para os respectivos elétrons $|1 / 2\rangle$ e buracos-pesados $|-3 / 2\rangle$. Como a constante da interação de troca é bem maior para buracos que para elétrons, os efeitos são mais pronunciados no potencial da banda de valência. Isto reflete nas energias de sub-banda e nas funções envelope, obtidas resolvendo a Eq. (3.1) e apresentadas na Fig. 3.4.

As DMHs mencionadas no parágrafo anterior são especialmente interessantes para o estudo de aglomeração e diluição de impurezas magnéticas 
justamente porque elas são capazes de explorar os limites de validade dos modelos para estes efeitos. Isto porque a maioria das amostras possui $x_{p} \geq 0.25$, i.e., numa faixa de concentração que os modelos de aglomeração divergem entre si (cf. Fig. 2.4 na pág. 26). Adicionalmente, a aleatoriedade da distribuição de Mn pode não ser mais válida, comprometendo também o uso dos modelos apresentados na Sec. 2.4.

Adicionalmente, as amostras com uma menor concentração planar de $\mathrm{Mn}$ - e portanto mais adequadas para os modelos de aglomeração ${ }^{4}$ - têm um número maior de barreiras, $N_{b}=12$ e 24. Devido a diluição, seus perfis de concentração se sobrepõem de tal forma que o perfil resultante pode estar além da descrição em termos de perfis exponenciais e de função erro apresentada na Sec. $2.4^{5}$

Apesar das limitações dos modelos disponíveis, aqui eu mostro que a inclusão de efeitos de diluição melhoram os resultados da Ref. [39] ao obter um acordo quantitativo com os dados experimentais.

\subsection{Resultados: desdobramento de spin}

Os desdobramentos de spin relevantes medidos experimentalmente envolvem pares de elétrons e buracos pesados (a energia do laser é ajustada de forma a induzir apenas estas transições). Estes pares têm componentes de momento angular total \pm 1 e são diretamente medidos por espectroscopia magnetoóptica com luz circularmente polarizada [RI, RII]. As transições ópticas envolvem apenas a primeira sub-banda de elétrons e buracos pesados [cf. Fig. 3.1]. Na presença de um campo magnético, a energia de transição para o par com polarização +1 é

$$
E_{h h, e}^{+1}(B)=\varepsilon_{1,-1}^{e}(B)-\varepsilon_{1,1}^{h h}(B)
$$

e para o par com polarização -1

$$
E_{h h, e}^{-1}(B)=\varepsilon_{1,1}^{e}(B)-\varepsilon_{1,-1}^{h h}(B)
$$

\footnotetext{
${ }^{4} \mathrm{~A}$ utilização da estatística de aglomerados para descrever o efeito de aglomeração antiferromagnética é válida (bulk) para $x \lesssim 0.1$, cf. Sec. 2.2 .

${ }^{5}$ Os perfis modelo da Sec. 2.4, Eqs. (2.24), (2.25) e (2.26), fazem uma sobreposição linear das concentrações. Em uma primeira aproximação esta é uma hipótese razoável. Entretanto, quando há muita sobreposição entre camadas de impurezas vizinhas, o modelo termodinâmico para segregação, por exemplo, resulta um perfil mais assimétrico que o modelo que eu adoto na Eq. (2.24). Mesmo assim, os perfis modelos apresentados no Cap. 2 fornecem resultados razoáveis em todas as partes desta tese em que são utilizados.
} 
Denotando por $E_{h h, e}^{0}$ a energia de transição $h h-e$ a campo magnético nulo, define-se o desdobramento induzido por troca $s p-d$ para os pares \pm 1 como

$$
\Delta E^{ \pm}(B)=E_{h h, e}^{ \pm 1}(B)-E_{h h, e}^{0} .
$$

Os experimentos de fotoluminescência das Refs. [RI, RII] mostram que, para um dado campo magnético fixo $e$ uma dada quantidade total fixa de Mn, os desdobramentos de spin $\Delta E$ aumentam com o número $N_{b}$ de camadas de Mn no poço, Figs. 3.2 e 3.3. ${ }^{6}$ De acordo com RI, este ordenamento particular é consistente com a ideia de diminuição da aglomeração antiferromagnética com a diluição da concentração de Mn. Esta interpretação é corroborada pelo cálculo teórico da Ref. [39], na qual tanto o desdobramento de spin calculado, quanto o ordenamento com o número de planos, estão de acordo com os achados experimentais. $\mathrm{Na}$ Ref. [39], a inclusão de efeitos de aglomeração via uma concentração efetiva de Mn é crucial para obter o ordenamento correto observado experimentalmente. Entretanto, a diluição ocorre apenas no particionamento do conteúdo de $\mathrm{Mn}$ em várias barreiras, mas utilizando perfis de concentração quadrados (ideais).

No que segue eu investigo a influência dos efeitos de diluição da concentração de Mn sobre o desdobramento de spin gigante utilizando perfis nãoideais. Eu comparo os resultados usando os perfis ideal $\left(\wp_{\text {sqr }}\right)$, de interdifusão $\left(\wp_{\text {erf }}\right.$ ) e de segregação $\left(\wp_{\text {exp }}\right)$. Os cálculos de $\Delta E$ são para vários números de barreiras $N_{b}=1,3,6,9,12,15,18$ e 40 (limite DMS) e comprimentos característicos $\ell$ para interdifusão e segregação.

Primeiro eu mostro a importância de se considerar a aglomeração antiferromagnética. Os parâmetros utilizados no cálculo (Tabela 3.1 e $T+T_{0}=5 \mathrm{~K}$ ) são correspondentes aos resultados experimentais da Fig. 3.2 [RI]. Os resultados apresentados na Fig. 3.5 não consideram aglomeração antiferromagnética - utilizei o perfil de concentração nominal $x(z)$, Eq. (2.28), na parte magnética do potencial (3.2). ${ }^{7}$ Três aspectos nestas curvas contradizem os resultados experimentais:

1. O desdobramento de spin chega a ser $\sim 4$ vezes superior àqueles da Fig. 3.2.

2. Enquanto o ordenamento experimental é tal que $\Delta E$ cresce com $N_{b}$ para os dois ramos $(\sigma= \pm 1)$, aqui ocorre uma inversão para o ramo inferior, com $\Delta E^{+}$decrescendo com $N_{b}$.

\footnotetext{
${ }^{6}$ A quantidade total de Mn em cada amostra é de 3 ML em [RI, RII]. Estas três monocamadas de Mn são distribuídas, em cada amostra, num número diferente de barreiras $\left(N_{b}\right)$. Assim, cada barreira tem uma concentração planar diferente, $x_{p}=3 / N_{b} \mathrm{ML}$, mantendo a concentração total constante.

${ }^{7}$ Nesta primeira análise eu mantive o comprimento característico de diluição fixo em $\ell=3 \AA$; mais adiante nesta seção eu uso $\ell$ como parâmetro de ajuste com os dados experimentais.
} 


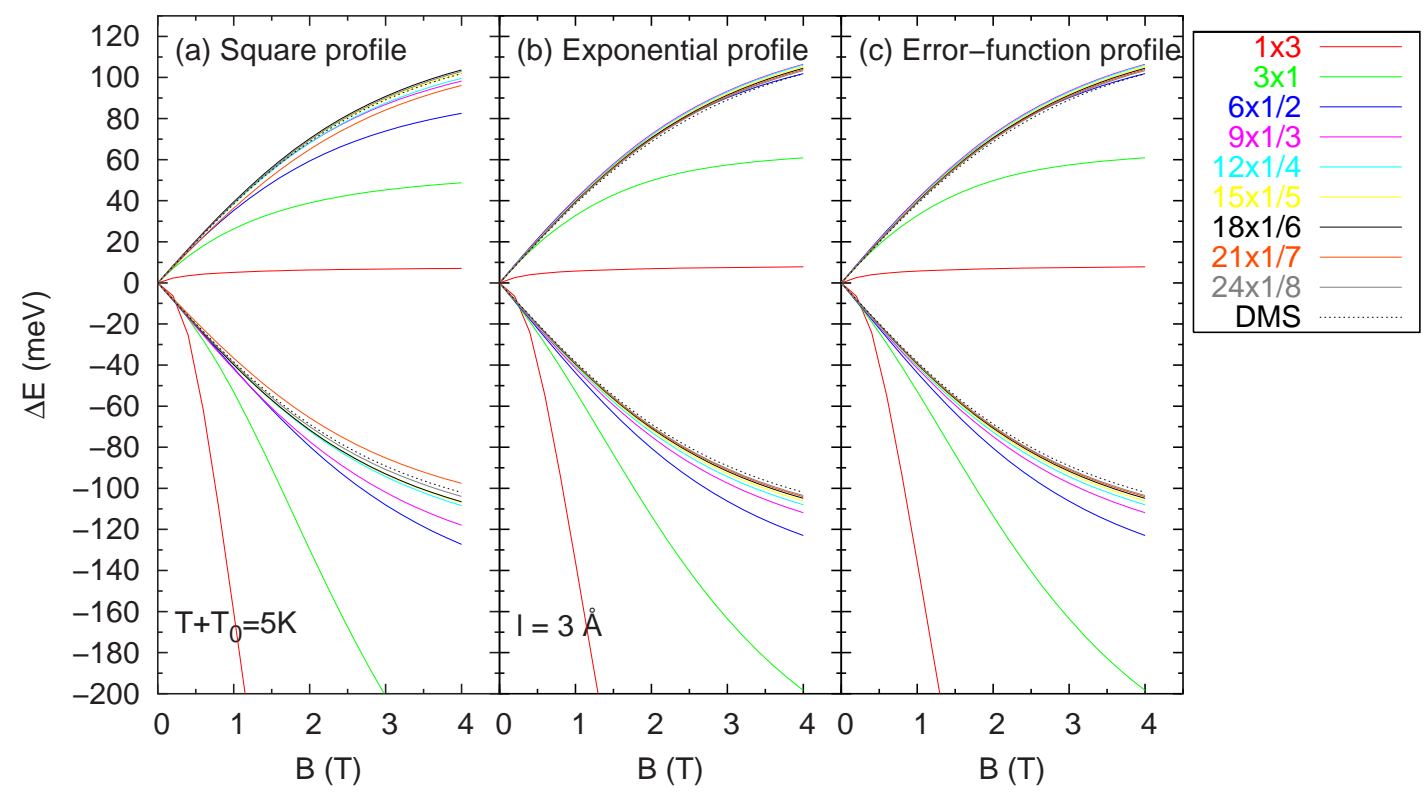

Figura 3.5: Desdobramento de spin usando concentração nominal (sem aglomeração antiferromagnética) para os casos de perfil ideal (a), de segregação (b), e de interdifusão (c). A magnitude de $\Delta E$ é $\sim 4$ vezes superior ao observado experimentalmente (cf. Figs. 3.2 e 3.3) e o ordenamento (maior $\Delta E$ corresponde a maior $\left.N_{b}\right)$ é invertido para polarização $\sigma=+1(\Delta E<0)$.

3. A inclusão dos perfis de diluição [painéis (b) e (c)] apenas diminui as diferenças entre os $\Delta E$ 's para $N_{b} \geq 6$, mais acentuado no ramo superior $(\sigma=-1)$. Note que na Fig. 3.2 as curvas para $N_{b}=6$ e 24 são bem separadas. $^{8}$

A Fig. 3.6 mostra os resultados levando em consideração aglomeração antiferromagnética (apenas singletos, modelo $P_{1}$ ); a parte magnética do potencial (3.2) usa o perfil de concentração efetivo $\bar{x}(z)$ definido na Eq. (2.30). O painel (a) para o caso de perfil quadrado reproduz o resultado da Ref. [39]: (i) a magnitude dos $\Delta E$ 's é apenas um pouco superior aos dados experimentais, (ii) o ordenamento está correto nos dois ramos, e (iii) as curvas para diferentes $N_{b}$ estão mais espaçadas entre si, quando comparadas com o caso de concentração nominal. Este resultado da Ref. [39] demonstrou pela primeira vez que a aglomeração antiferromagnética é responsável pelo ordenamento em função do número de barreiras. Entretanto, as configurações e $1 \times 3 \mathrm{ML}$ e $3 \times 1 \mathrm{ML}$ resultam $\Delta E=0$,

\footnotetext{
${ }^{8}$ Para os casos $1 \times 3 \mathrm{ML}$ e $3 \times 1 \mathrm{ML}$ da Fig. 3.5 (concentração nominal apenas), $x_{p}=1$ e a contribuição de troca sp-d para o potencial dependente de spin é máxima. Este efeito é mais acentuado para os buracos-pesados $|+3 / 2\rangle$, localizando fortemente as suas funções de onda sobre os "potencias atrativos" e abaixando a sua energia. Para se ter uma ideia, veja a Fig. 3.4 na qual o perfil de potencial é usando concentrações efetivas; para concentração nominal as alturas das barreiras magnéticas ficam bem maiores. Assim, no caso de concentrações nominais (sem aglomeração antiferromagnética), a dependência da energia $\varepsilon_{1,1}^{h h} \operatorname{com} B$ é responsável pela variação acentuada de $\Delta E^{+}$para $x_{p}=1$.
} 


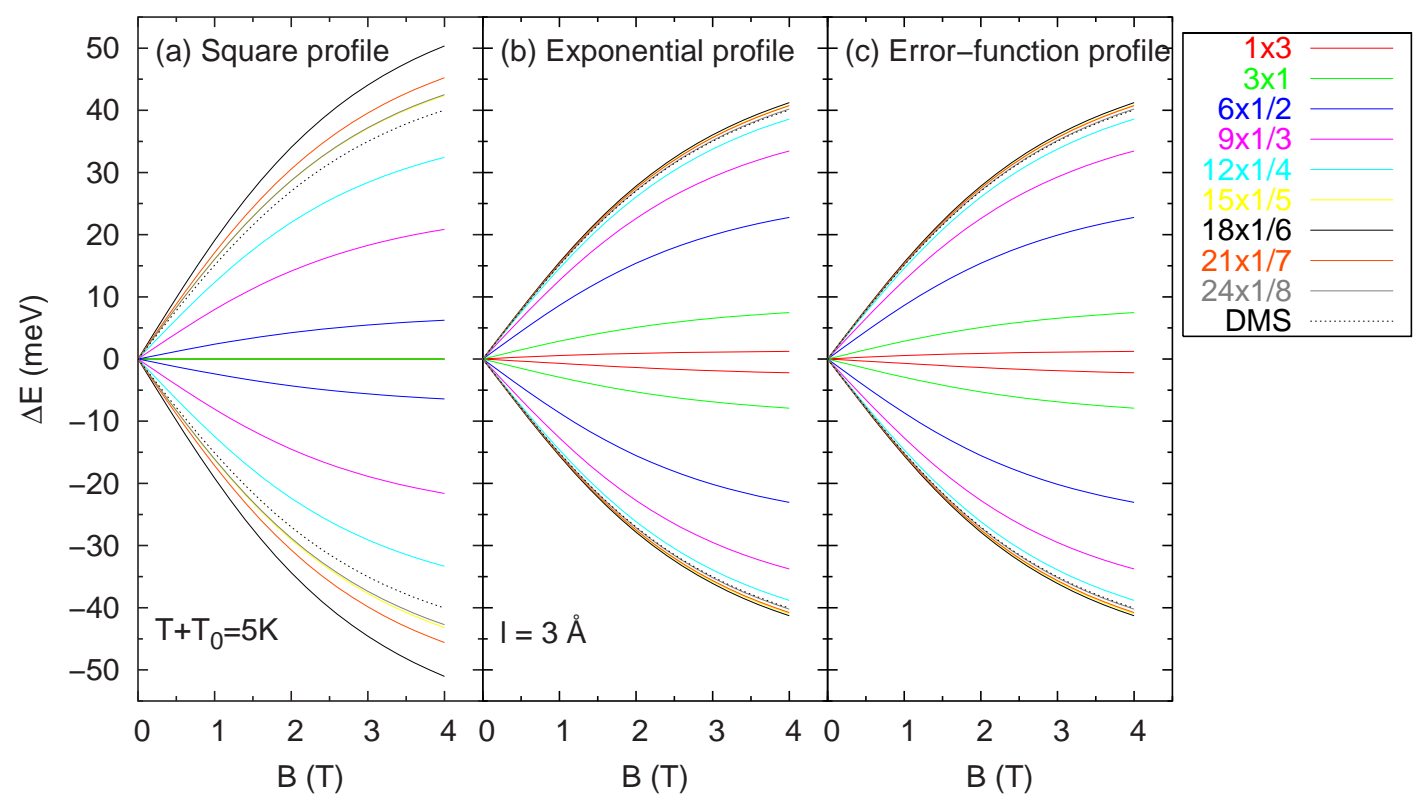

Figura 3.6: Desdobramento de spin usando concentração efetiva para os perfis ideal (a), de segregação (b), e de interdifusão (c), os dois últimos com $\ell=3 \AA$. Para o caso de perfil quadrado (a), os valores calculados para $\Delta E$ reproduzem os resultados da Ref. [39]. Neste caso, a magnitude de $\Delta E$ é um pouco maior que nos dados experimentais (cf. Figs. 3.2 e 3.3). Já para os casos utilizando perfis não-ideais [(b) e (c)], a magnitude de $\Delta E$ compara com o experimento. O ordenamento (maior $N_{b}$ corresponde a maior $\Delta E$ ) é correto para as duas polarizações $(\sigma= \pm 1)$. Para os casos com maior número de barreiras $\left(N_{b} \geq 15\right)$, as curvas de $\Delta E$ para o perfil ideal são bem distintas, enquanto elas se sobrepõe próximas ao valor para DMS nos casos de segregação e interdifusão, como observado experimentalmente.

contrário aos resultados experimentais que fornecem valores pequenos, mas não nulos, Fig. 3.2.

Já o desdobramento de spin usando perfis não-ideais para a concentração de Mn, painéis (b) e (c) da Fig. 3.6, reproduz várias características observadas experimentalmente.

1. A magnitude dos $\Delta E$ 's para $N_{b}$ maiores diminui um pouco com relação ao caso de perfil quadrado, aproximando-se mais do valor experimental $(\sim 40 \mathrm{meV})$.

2. A diluição resulta em $\Delta E \neq 0$ para os casos mencionados no parágrafo anterior, $1 \times 3 \mathrm{ML}$ e $3 \times 1 \mathrm{ML}$.

3. Adicionalmente, $\Delta E$ para $N_{b} \leq 12$ aumenta quando comparado com o caso ideal, devido a diminuição de aglomeração devido a diluição. Para $N_{b}>12$ a sobreposição em barreiras vizinhas cancela a diminuição da aglomeração 


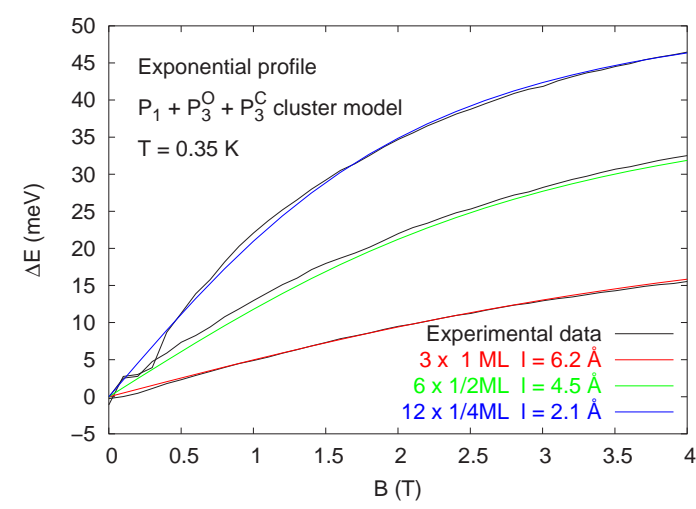

Figura 3.7: Resultados teóricos para o desdobramento de spin a $T=0.35 \mathrm{~K}$ (curvas coloridas). As curvas pretas são os dados experimentais de RII. Para esta figura foi usado o modelo de aglomeração com singletos e tripletos, e diluição por segregação (perfil exponencial, com comprimentos característicos indicados na legenda). Os demais parâmetros estão indicados na Tabela (3.2). As temperaturas efetivas são aquelas indicadas na legenda da Fig. 3.3. (O comportamento da curva experimental $12 \times 1 / 4$ ML em $0.1>B<0.5$ aparenta ser proveniente de erros experimentais na medida.)

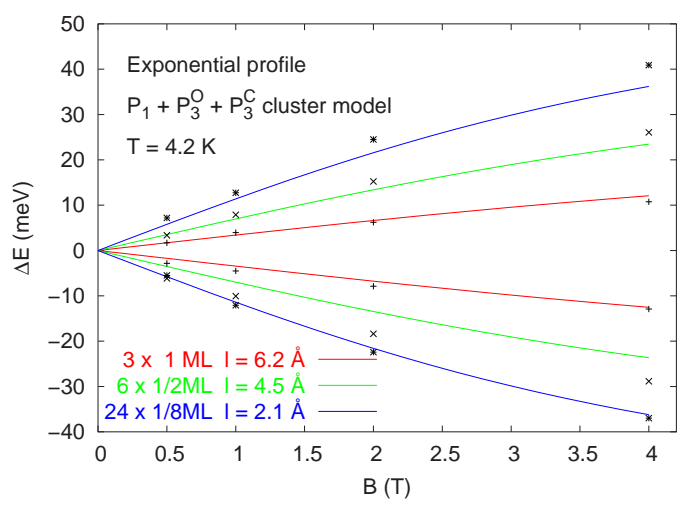

Figura 3.8: Resultados teóricos para o desdobramento de spin a $T=4.2 \mathrm{~K}$ (curvas coloridas). Aqui eu uso os comprimentos característicos ajustados para reproduzir os dados experimentais a $T=0.35 \mathrm{~K}$ (Fig. 3.7). Os pontos são os dados experimentais de RI. Como não há um ajuste de $\ell$ para o caso $24 \times 1 / 8 \mathrm{ML}$ (não foram divulgados dados experimentais para este caso a $T=0.35 \mathrm{~K}$ ), foi utilizado o valor obtido para o caso $12 \times 1 / 4 \mathrm{ML}$ a $T=0.35 \mathrm{~K}$.

devido a diluição, e o $\Delta E$ resultante aproxima-se do caso DMS.

Estes resultados usando perfis não-ideais descrevem os achados experimentais de $\mathrm{RI}$ razoavelmente bem.

Os resultados da Ref. [RII] também foram reproduzidos, mas agora usando o comprimento característico $\ell$ como parâmetro de ajuste. Estes resultados, apresentados na Fig. 3.7, usam a função perfil $\wp_{\exp }(z)$ com o modelo de aglomeração de singletos mais tripletos $\left(P_{1}+P_{3}\right)$. Neste caso, $T=0.35 \mathrm{~K}$ e os valores de $T_{0}$ são os mesmos usados na interpolação com a função de Brillouin da Fig. 3.3. Os valores de $\ell$ que melhor ajustam as curvas experimentais estão indicados na legenda da figura. Estes mesmos comprimentos característicos foram então usados para calcular os desdobramentos de spin a $T=4.2 \mathrm{~K}$, Fig. 3.8, para as mesmas amostras [RI]. Eu usei as mesmas temperatura efetivas anteriores. Como RII apresenta dados para a amostra $12 \times 1 / 4 \mathrm{ML}$, e RI apresenta dados para a amostra $24 \times 1 / 8 \mathrm{ML}$, eu usei os parâmetros da primeira $\left(N_{b}=12\right)$ para simular a segunda $\left(N_{b}=24\right)$ a $T=4.2 \mathrm{~K}$ - como a sobreposição da barreiras é grande quando $N_{b}$ é elevado, os resultados tanto para 12 quanto para 24 barreiras não 
Tabela 3.2: Comprimentos característicos que melhor ajustam os dados experimentais de Crooker et al. [RII] para $\Delta E \times B$. Os ajustes foram feitos usando os modelos de perfis de concentração indicados no texto. Cada valor de $\ell$ foi determinado de forma a reproduzir os valores de saturação $\Delta E_{\text {sat }}$ em campos altos.

\begin{tabular}{|c|c|c|c|c|c|c|c|c|}
\hline \multirow{3}{*}{$\begin{array}{r}\text { Aglomerado } \rightarrow \\
\wp(z) \rightarrow\end{array}$} & \multicolumn{8}{|c|}{ Comprimento característico $\ell(\AA)$} \\
\hline & \multicolumn{2}{|c|}{$x_{N N}$} & \multicolumn{2}{|c|}{$P_{1}$} & \multicolumn{2}{|c|}{$P_{1}+P_{3}^{O}+P_{3}^{C}$} & \multicolumn{2}{|c|}{$P_{5}$} \\
\hline & $\wp_{\exp }$ & $\wp_{\text {erf }}$ & $\wp_{\exp }$ & $\wp_{\text {erf }}$ & $\wp_{\exp }$ & $\wp_{\text {erf }}$ & $\wp_{\exp }$ & $\wp_{\text {erf }}$ \\
\hline $3 \times 1 \mathrm{ML}\left(\Delta E_{\mathrm{sat}}=24.6 \mathrm{meV}\right)$ & 3.3 & 4.2 & 6.6 & 6.9 & 6.2 & 6.6 & 1.9 & 3.0 \\
\hline $6 \times 1 / 2 \mathrm{ML}\left(\Delta E_{\mathrm{sat}}=40.6 \mathrm{meV}\right)$ & 1.9 & 2.7 & 5.7 & 5.2 & 4.5 & 4.6 & 1.9 & 2.7 \\
\hline $12 \times 1 / 4 \mathrm{ML}\left(\Delta E_{\mathrm{sat}}=52.5 \mathrm{meV}\right)$ & $0.0^{\dagger}$ & $0.0^{\dagger}$ & $>10^{\ddagger}$ & $>5.8^{\ddagger}$ & 2.1 & 2.3 & $0.0^{\dagger}$ & $0.0^{\dagger}$ \\
\hline
\end{tabular}

deve diferir muito [cf. Fig. 3.6(b)].

O perfil de interdifusão e outros modelos de aglomerados também foram usados para ajustar os dados experimentais de RII, e os resultados são comparados na Tabela 3.2. Os valores de $\ell$ variaram na faixa de 0 a $10 \AA$. Para $12 \times 1 / 4 \mathrm{ML}$, só foi possível encontrar valores para $\ell$ para o modelo de aglomerados com singletos e tripletos $\left(P_{1}+P_{3}\right)$. Para os modelos $x_{N N}$ e $P_{5}, \ell=0.0$ correspondeu ao menor desdobramento de spin encontrado, mas ainda maior que o valor experimental. Em contraste, os valores encontrados para $\ell$ com o modelo $P_{1}$ resultaram em $\Delta E$ 's menores que o experimental.

Como mencionei anteriormente, as concentrações nominais $x_{p}$ usadas nestas amostras são relativamente altas, podendo estar além do limite de validade dos modelos estatísticos para aglomerados. Porém, o resultado de que apenas o modelo intermediário $P_{1}+P_{3}$ possibilitou encontrar valores de $\ell$ que ajustam os resultados experimentais é bastante sugestivo. Além disso, ${ }^{9}$ o modelo $P_{1}$ aparentemente superestima a aglomeração que ocorre na amostra, uma vez que ele considera que apenas singletos contribuem para a magnetização média do Mn - portanto, ao calcular $\Delta E$ preciso de $\ell$ 's maiores para diminuir o efeito de aglomeração e reproduzir os dados experimentais (cf. Tabela 3.2). Enquanto isso, o modelo $P_{5}$ subestima a aglomeração ao supor que todos os spin que não estão isolados, em pares, ou em tripletos, estão em quintetos com spin $5 / 2$ [Eq. 2.5] - consequentemente, no cálculo de $\Delta E$ os valores para $\ell$ são menores para que a diluição não diminua muito a aglomeração antiferromagnética. Sendo $P_{1}+P_{3}$ um modelo intermediário, está consistente o fato dele ter sido capaz de ajustar os dados experimentais.

\footnotetext{
${ }^{9}$ Veja a Fig. 2.4(c) na pág. 26, que compara os vários modelos de aglomerados, para facilitar o entendimento do argumento que segue.
} 


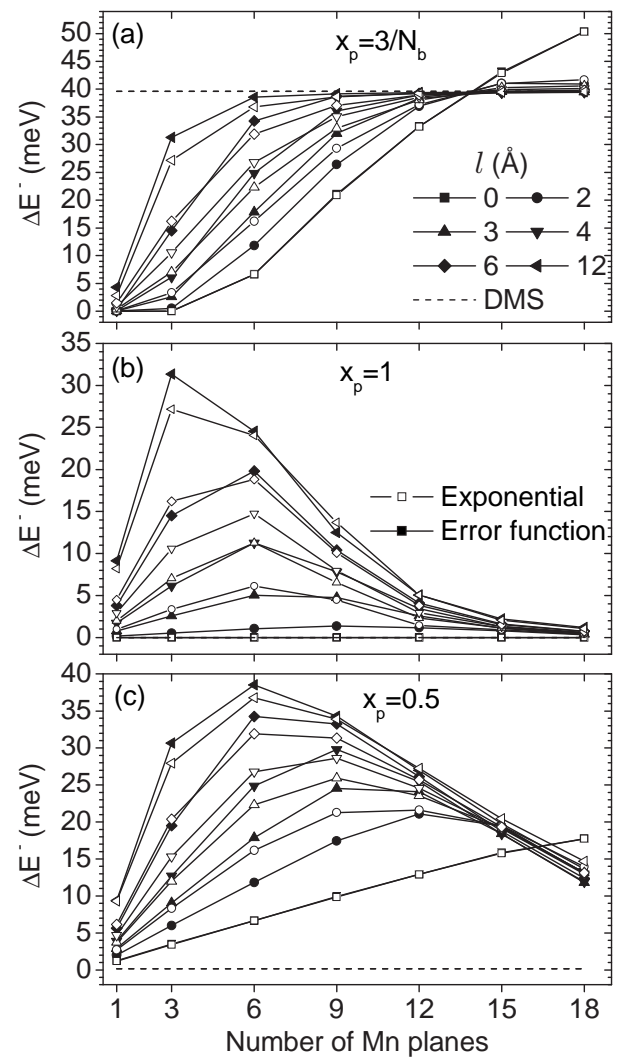

Figura 3.9: Desdobramento $\Delta E^{-}$a $B=4 \mathrm{~T}$ como função do número de planos magnéticos $N_{b}$, para vários comprimentos característicos $\ell$, tanto para interdifusão (perfil de função-erro) e segregação (perfil exponencial). Em (a) a quantidade total de Mn é mantida constante e distribuída entre as barreiras $\left(x_{p}=3 / N_{b}\right)$, como em RI e RII. Neste caso a aglomeração de spins das impurezas não diminui $\Delta E^{-}$devido o particionamento da concentração para $N_{b}$ 's maiores. Nos painéis (b) e (c) cada camada magnética tem a mesma concentração nas configurações com $N_{b}$ 's diferentes. Neste caso a aglomeração antiferromagnética diminui $\Delta E^{-}$ quando há muitas barreiras.

Competição entre diluição e aglomeração Para investigar a interconexão entre o número $N_{b}$ de planos de $\mathrm{Mn}$ e o comprimento característico $\ell$ de segregação e interdifusão, a Fig. 3.9 apresenta o desdobramento de spin induzido por troca $\Delta E^{-}$como função de número de barreiras e para vários comprimentos característicos.

- Para o caso em que $x_{p}=3 / N_{b}$ [Fig. 3.9(a)], o desdobramento aumenta com $N_{b}$ e $\ell$, aproximando do caso DMS. Isto acontece porque, no caso de concentração constante, quanto maior $N_{b}$ menor é a concentração em cada barreira. Assim, há menos emparelhamentos Mn-Mn, o que corresponde a uma concentração efetiva maior. À medida que o comprimento característico cresce, este efeito aumenta, dominando até que os perfis de barreiras 
vizinhas comecem a se sobrepor. Neste regime o aumento da aglomeração antiferromagnética diminui a concentração efetiva e compensa a diluição devido a valores maiores de $N_{b}$ e $\ell$. Note que, para $\ell$ pequeno e $N_{b}=15$ e 18, $\Delta E_{D M H}^{-}>\Delta E_{D M S}^{-}$, ou seja, para uma mesma quantidade de Mn, o desdobramento de spin em DMHs pode ser maior que em DMSs.

- Já para o caso em que as concentrações nas barreiras são mantidas constantes [Fig. 3.9(b) e (c)], o aumento do número de barreiras inicialmente aumenta $\Delta E^{-}$(mais Mn, mais intenso é o efeito de troca sp-d ). Entretanto, para $N_{b}$ 's maiores, começa a ocorrer muita sobreposição entre barreiras vizinhas, o que acaba por aumentar a aglomeração e, consequentemente, diminuir a concentração efetiva de íons contribuindo para a interação de troca.

- Diferenças entre perfis de função-erro e exponencial são mais pronunciadas nas configurações com 3, 6 e 9 barreiras, principalmente nos casos ilustrados na Fig. 3.9(b) e (c). Para $N_{b}=1$, os desdobramentos de spin calculados são muito pequenos para mostrar uma distinção clara entre os perfis. Para $N_{b} \geq 12$ o exato perfil de potencial não é mais tão importante porque a sobreposição entre as barreiras vizinhas é dominante; isto torna os perfis não-ideais indistinguíveis.

Discussões Nesta seção eu mostrei como a inclusão de efeitos de aglomeração antiferromagnética dos íons de Mn, mais a diluição do perfil de concentração destes íons, permitiram reproduzir os resultados experimentais de RI e RII, complementando o trabalho inicial da Ref. [39].

Entretanto, a questão se segregação ou interdifusão é dominante nestes sistemas permanece aberta. As simulações dos desdobramentos de spin permitem estimar que a faixa de comprimentos característicos está entre $2 \AA$ e $6 \AA$, mas não permite distinguir entre os dois tipos de perfis. Uma investigação experimental sistemática sobre o tipo de perfil dominante nestas DMHs poderia ajudar a elucidar este problema. Por exemplo, crescer um conjunto de amostras, cada uma com apenas uma monocamada magnética dentro de um poço quântico, mas variando a concentração nominal de $\mathrm{Mn}$ entre elas, de $x_{p}=0$ a $x_{p}=1$. Medidas de magnetização ou desdobramento Zeeman nestas amostras forneceriam uma contrapartida da Eq. (2.1) para arranjos 2D, que por sua vez seria uma referência para um estudo teórico, seja criando modelos fenomenológicos como $x_{N N}$ de Grieshaber et al. [61], seja analisando usando a estatística de aglomerados. 


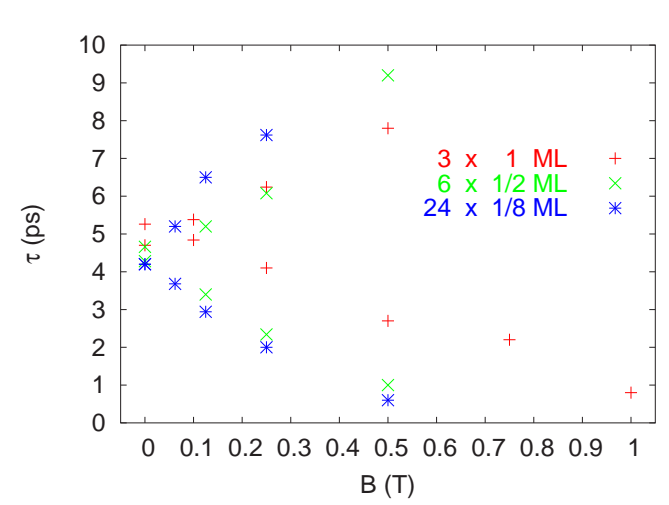

Figura 3.10: Tempo de espalhamento de spin experimental em função do campo magnético para as três configurações indicadas [RI]. Os valores são baseados em medidas de polarização resolvidas no tempo na configuração de rotação de Faraday. O ramo superior (inferior) corresponde a excitação com polarização $\sigma=+1(\sigma=-1)$. Note que (i) a $B=0$ os valores de $\tau$ são aproximadamente iguais, e (ii) o desdobramento a $B=0.5 \mathrm{~T}$ é $\sim 8 \mathrm{ps}$.

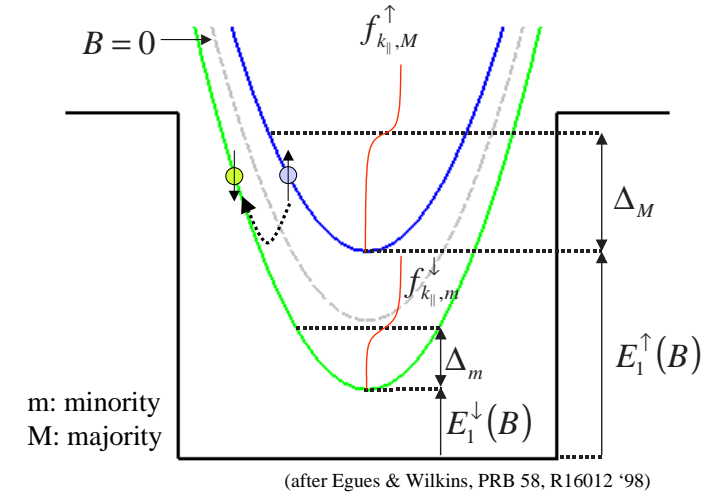

Figura 3.11: Ilustração do mecanismo de espalhamento de spin induzido pela interação de troca $s p-d$. As sub-bandas de elétrons de spin para cima (majoritário) e spin para baixo (minoritário) estão desdobradas $(\sim 7 \mathrm{meV}$ a $B=$ $1 \mathrm{~T})$ devido ao efeito Zeeman gigante. A parábola tracejada indica o caso a $B=0$. Em cada sub-banda os elétrons são distribuídos de acordo com as funções de Fermi $f_{M}^{\uparrow}$ e $f_{m}^{\downarrow}$ com temperatura eletrônica $T_{e}$. Os elétrons podem sofrer um espalhamento de spin e mudar de sub-banda. O espaço de fase disponível para as transições $\uparrow \downarrow$ e $\downarrow \uparrow$ varia com o campo magnético via as energias das sub-bandas $\varepsilon_{1}^{\sigma_{z}}$ presentes nas funções de Fermi.

\subsection{Resultados: tempo de espalhamento de spin}

Aqui eu extendo o cálculo do tempo de espalhamento de spin da Ref. [39] para DMHs ideais incluindo efeitos de segregação e interdifusão. Considero processos de espalhamento de spin induzidos pela interação de troca $s$-d conectando as sub-bandas de spin de elétrons.

Note porém, como enfatizado na Ref. [39], que este mecanismo não é necessariamente o mais relevante em DMHs. O espalhamento de spin ocorre também em amostras que não contêm Mn, apesar que o desdobramento em dois ramos (cf. Fig. 3.10) só ocorre nas amostras magnéticas. Isto significa que a interação de troca $s-d$ dos elétrons com os spins de Mn não é necessariamente a principal causa do espalhamento de spin. O desdobramento de spin nos tempos de espalhamento pode estar apenas indiretamente ligado à interação de troca via a dependência das fatores de Fermi com as energias das sub-bandas de spin ("preenchimento do espaço de fase"). De fato, os resultados desta seção reforçam 
aqueles da Ref. [39] neste sentido. De qualquer maneira, considerar o espalhamento de spin devido a interação de troca fornece um modelo de espalhamento ("spin-flip") simples, através do qual pode-se investigar a forte dependência dos tempos de espalhamento de spin com o campo magnético em termos dos fatores de Fermi.

Esta parte do trabalho focalizou particularmente nos efeitos dos perfis não-ideais no desdobramento característico de $\tau_{s f}$ discutido na Ref. [39]. Isto é, experimentalmente sabe-se que as transições "spin para cima para spin para baixo" e "spin para baixo para spin para cima" em amostras magnéticas (DMS e DMH) têm um desdobramento em dois ramos que aumenta com o campo, como ilustrado na Fig. 3.10. Adicionalmente, independente da amostra (diferentes configurações $N_{b} \times x_{p} \mathrm{ML}$ ), as curvas de $\tau_{s f} \times B$ têm origem nos mesmos valores a $B=0$. Ao considerar diluição, é possível obter esta última característica, ao contrário do cálculo anterior [39] que usou perfis ideais (quadrados).

Assim, aqui eu considero processos de espalhamento de spin conectando sub-bandas de spin desdobradas por troca sp-d [39], e estudo os efeitos dos perfis de segregação e interdifusão propostos na Sec. 2.5 sobre os tempos de espalhamento de spin. Admito que em cada sub-banda os elétrons são distribuídos de acordo com a distribuição de Fermi-Dirac com temperatura $T_{e}$, como ilustrado na Fig. 3.11. ${ }^{10}$ Dois casos, experimentalmente viáveis [RI], são relevantes nestes cálculos do tempo de espalhamento (veja a Fig. 3.11):

1. $\uparrow \downarrow$ : spin para cima majoritário espalha para spin para baixo minoritário; e

2. $\downarrow \uparrow$ : spin para baixo majoritário espalha para spin para cima minoritário.

\subsubsection{Modelo teórico para $\tau_{s f}$}

A dependência dos tempos de espalhamento de spin com o campo magnético foi investigada para DMHs por Egues e Wilkins [39]. ${ }^{11}$ A generalização daqueles resultados para levar em consideração o efeito de diluição os tempos de espalhamento de spin $\tau_{s f}^{\uparrow \downarrow}$ é direta, apenas substituindo os perfis quadrados usados em

\footnotetext{
${ }^{10} \mathrm{~A}$ temperatura dos elétrons $T_{e}$ é diferente da temperatura da rede $T_{L}$ pois, na escala de tempo em que ocorre o espalhamento de spin $(\sim \mathrm{ps})$, os elétrons fotogerados não termalizam com a rede.

${ }^{11} \mathrm{O}$ cálculo de $\tau_{s f}$ efetuado em [39] segue as mesmas linhas de Kossut [81] e Bastard e Chang [82], mas incluindo o efeito do campo magnético externo e fatores de preenchimento de fase (função de Fermi). Este cálculo vai além da aproximação de campo médio usada aqui para descrever a magnetização dos íons de Mn (função de Brillouin). Veja a Ref. [83] para um cálculo detalhado.
} 
[39] por perfis com diluição (Sec. 2.5), resultando em (veja o Apêndice A)

$$
\frac{1}{\tau_{s f}^{\uparrow \downarrow}\left(\varepsilon_{k_{\|}}\right)}=F(B) \times \Gamma\left(N_{b}, \ell\right) \times\left[1-f_{m}^{\downarrow}\left(\varepsilon_{k_{\|}}\right)\right],
$$

na qual

$$
\begin{gathered}
F(B)=\frac{m \Omega_{0}}{4 a_{0} \hbar^{3}}\left(N_{0} \alpha\right)^{2}\left\{S(S+1)-\left\langle S_{z}^{2}\right\rangle-\left\langle S_{z}\right\rangle\right\}, \\
\Gamma\left(N_{b}, \ell\right)=a_{0} \int_{L_{0}}\left|\chi_{1}^{\uparrow}(z)\right|^{2}\left|\chi_{1}^{\downarrow}(z)\right|^{2} \bar{x}(z) \mathrm{d} z, \\
{\left[1-f_{m}^{\downarrow}\left(\varepsilon_{k_{\|}}\right)\right]=\frac{1}{\exp \left[-\left(\varepsilon_{1}^{\uparrow}+\varepsilon_{k_{\|}}-\mu_{m}^{\downarrow}\right) / k_{B} T_{e}\right]+1},}
\end{gathered}
$$

sendo $m$ a massa efetiva do elétron, $\Omega_{0}$ o volume da célula primitiva, ${ }^{12} a_{0}$ o raio de Bohr, $N_{0} \alpha$ a constante de troca para elétrons, e $S=5 / 2$ o spin do Mn. $\langle\cdots\rangle$ denota médias térmicas (na temperatura da rede ${ }^{13} T_{L}=5 \mathrm{~K}$ ) sobre as orientações dos spins do $\mathrm{Mn}, S_{z}=-5 / 2,-3 / 2, \cdots, 3 / 2,5 / 2$. A energia $\varepsilon_{k_{\|}}$é a energia inicial do elétron associada ao movimento transversal. $\mu_{M(m)}$ é o potencial químico de elétrons majoritários (M) e minoritários (m), dado por [39]

$$
\mu_{M(m)}^{\sigma_{z}}=\varepsilon_{1}^{e, \sigma_{z}}+k_{B} T_{e} \ln \left[\exp \left(\Delta_{M(m)}^{\sigma_{z}} / k_{B} T_{e}\right)-1\right]
$$

$\operatorname{com} \Delta_{M(m)}^{\sigma_{z}}$ os excessos de energia correspondentes à ocupação das sub-bandas majoritária $(\mathrm{M})$ e minoritária $(\mathrm{m})$ - c.f. Fig. $3.11 .^{14}$

Para a transição $\downarrow \uparrow$ é usada uma equação similar a Eq. (3.7), mas trocando $-\left\langle S_{z}\right\rangle$ por $\left\langle S_{z}\right\rangle$ em (3.8), utilizando o fator de Fermi apropriado [1 $\left.f_{m}^{\uparrow}\left(\varepsilon_{k_{\|}}\right)\right]$, e com a condição que a energia $\varepsilon_{k_{\|}}$é suficiente para permitir que um elétron com spin para baixo espalhe para a sub-banda com spin para cima, i.e.,

$$
\varepsilon_{1}^{\downarrow}(B)+\varepsilon_{k_{\|}} \geq \varepsilon_{1}^{\uparrow}(B)
$$

Antes de passar para os resultados de tempo de espalhamento eu faço uma rápida análise da contribuição de cada termo da Eq. (3.7).

Magnitude do tempo de espalhamento: $F(B)$ O primeiro termo na Eq. (3.7) contem a contribuição da interação com os spins de Mn para o espalhamento de spin. A sua dependência com o campo magnético vem das médias $\left\langle S_{z}^{2}\right\rangle$ e $\left\langle S_{z}\right\rangle$.

\footnotetext{
${ }^{12} \mathrm{O}$ volume da célula primitiva é $\Omega_{0}=a^{3} / 4$, com $a$ o parâmetro de rede. Aqui eu uso o valor para o $\mathrm{ZnSe}, a \simeq 5.67 \AA$.

${ }^{13}$ Veja a nota de rodapé 10 .

${ }^{14}$ Para temperatura nula $T_{L}=0$, o potencial químico é o próprio nível de Fermi, ou seja, $\mu_{M(m)}^{\sigma_{z}}=\varepsilon_{F, M(m)}^{\sigma_{z}}=\varepsilon_{1}^{e, \sigma_{z}}+\Delta_{M(m)}^{\sigma_{z}}$.
} 

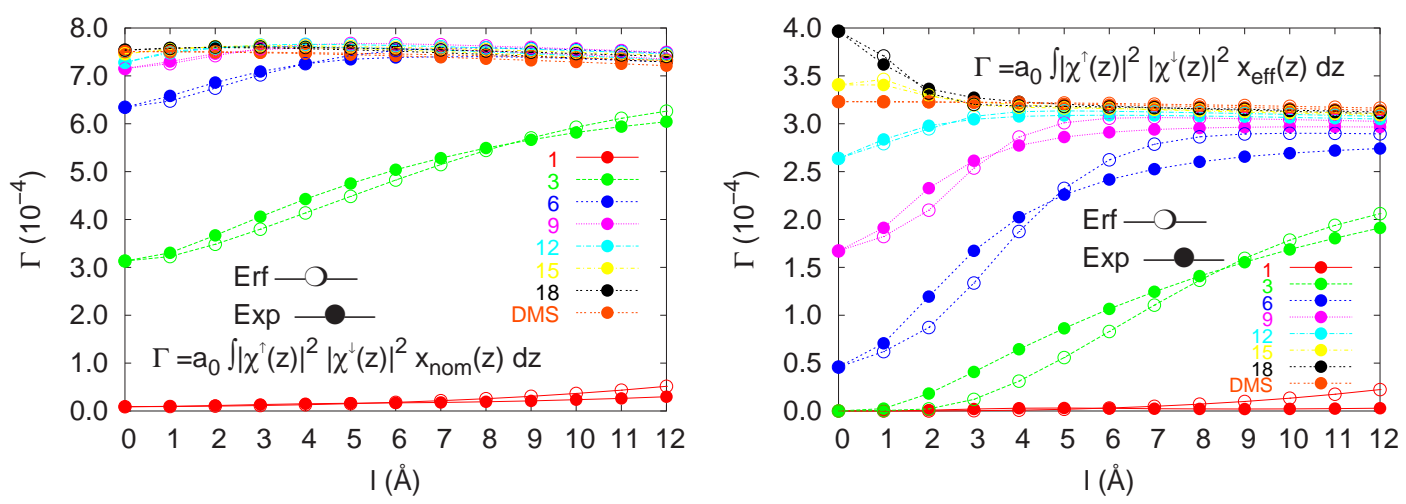

Figura 3.12: Fator de sobreposição $\Gamma\left(N_{b}, \ell\right)$ das funções de onda eletrônicas com o perfil de impurezas magnéticas. $\Gamma$ aumenta com $N_{b}$ e $\ell$, e os valores para diferentes tipos de perfis $\left(\wp_{\text {exp }}\right.$ e $\wp_{\text {erf }}$ ) são bastante próximos. A magnitude é maior no caso de concentração nominal (painel esquerdo) que no caso de concentração efetiva (painel direito) devido à aglomeração antiferromagnética. O efeito de diluição é responsável por aproximar as várias curvas para l's maiores.

Para se ter uma ideia melhor da magnitude deste termo, eu o reescrevo numa forma dimensionalmente mais interessante:

$$
F(B)=\frac{1}{2 \hbar} \frac{\left(N_{0} \alpha\right)^{2}}{R_{y}^{0}} \frac{\left(a / a_{0}\right)^{3}}{4}\left(m / m_{0}\right) \frac{1}{4}\left[S(S+1)-\left\langle S_{z}^{2}\right\rangle \mp\left\langle S_{z}\right\rangle\right],
$$

no qual $R_{y}^{0}$ é o Rydberg, $a_{0}$ é raio de Bohr, e $a$ é o parâmetro de rede. Escrito desta forma, é fácil notar que a sua dimensão é de inverso de tempo.

Usando que $N_{0} \alpha=260 \mathrm{meV}, m / m_{0}=0.16$, e $a=5.67 \AA$, tenho que

$$
\frac{1}{2 \hbar} \frac{\left(N_{0} \alpha\right)^{2}}{R_{y}^{0}} \frac{\left(a / a_{0}\right)^{3}}{4}\left(m / m_{0}\right) \cong 186 \mathrm{ps}^{-1}
$$

A parte que contem o spin $S=5 / 2$ e as médias $\left\langle S_{z}^{2}\right\rangle$ e $\left\langle S_{z}\right\rangle$ pode ser aproximada expandindo as médias no limite de $B$ pequeno, e mantendo apenas os termos constantes e lineares em $B$. O resultado é

$$
\frac{1}{4}\left[S(S+1)-\left\langle S_{z}^{2}\right\rangle \mp\left\langle S_{z}\right\rangle\right] \simeq \frac{1}{4}\left(\frac{35}{4}-\frac{35}{12} \pm \frac{35}{12} \frac{g_{M n} \mu_{B} B}{k_{B} T_{L}}\right)=\frac{35}{24}\left(1 \pm 0.67 \frac{B}{T_{L}}\right),
$$

na qual usei $g_{M n}=2, \mu_{B} / k_{B} \sim 0.67 \mathrm{~T}^{-1} \mathrm{~K}$, e $B$ e $T_{L}$ estão em unidades de Tesla e Kelvin, respectivamente. Assim, a magnitude de $F(B)$ é

$$
F(B) \simeq 271.25\left[1 \pm 0.67 B / T_{L}\right] \mathrm{ps}^{-1}
$$

Fator de sobreposição $\Gamma\left(N_{b}, \ell\right)$ Para ocorra spin-flip devido a interação de troca $s p-d$, é necessário que os orbitais iniciais e finais se sobreponham com os 
spins do Mn, o que diminui a probabilidade de espalhamento e aumenta o tempo característico. Este fator é dado justamente pelo termo (3.9)

$$
\Gamma\left(N_{b}, \ell\right)=a_{0} \int_{L_{0}}\left|\chi_{1}^{\uparrow}(z)\right|^{2}\left|\chi_{1}^{\downarrow}(z)\right|^{2} \bar{x}(z) \mathrm{d} z
$$

Em princípio, este termo varia com o campo magnético via as funções de onda. Entretanto, como estou considerando campos baixos, elas são pouco distorcidas (cf. as funções de onda para elétrons na Fig. 3.6). Na prática este fator depende mais do número de barreiras e de seus comprimentos característicos. A Fig. 3.12 mostra a dependência de $\Gamma$ com $\ell, N_{b}$ e o tipo de perfil, tanto para concentração nominal $x(z)$, quanto efetiva $\bar{x}(z)$. Note que, no caso extremo $1 \times 3 \mathrm{ML}$, o fator de sobreposição é bem pequeno. Neste caso, a barreira magnética "expulsa" as funções de onda, o que diminui consideravelmente a sobreposição.

Sabendo que $\Gamma \sim 1.5 \times 10^{-4}$, posso então fazer uma estimativa razoável do tempo característico de espalhamento (sem ainda considerar os fatores de Fermi). Usando a estimativa que fiz para $F(B)$ tenho

$$
\tau_{s f}^{-1} \sim 271.25 \times 1.5 \times 10^{-4}[1 \pm 0.67 B / T] \mathrm{ps}^{-1} \Rightarrow \tau \sim(24 \pm 16 B / T) \mathrm{ps}
$$

Para comparar esta estimativa de $\tau_{s f}$ com os tempos de espalhamento extraídos dos experimentos $\tau_{s f}^{e x p}$ (Fig. 3.10), é preciso considerar a técnica experimental utilizada e a definição utilizada para $\tau_{s f}^{\text {exp }}$. O tempo de espalhamento da Ref. [RI] está relacionado com o decaimento da polarização do gás de elétrons, e é extraído dos experimentos usando uma exponencial simples, $\exp \left(-t / \tau_{s f}^{\exp }\right)$. Entretanto, da forma que foi calculado, o tempo de espalhamento teórico está relacionado com a polarização por $P_{M}^{\uparrow}(t) \simeq 1-t /\left(\tau_{s f}^{\uparrow \downarrow} / 2\right)$, para o caso de elétron $|\uparrow\rangle$ majoritário [83]. Assim, eu preciso dividir por dois o tempo de espalhamento teórico para comparar com o tempo experimental [39, 83].

Considerando então a diferença entre os $\tau_{s f}$ 's teóricos e experimentais, a estimativa (3.17) corresponde um tempo de espalhamento a $B=0$ de $\sim 12$ ps, um pouco maior que o valor experimental ( $\sim 5$ ps, Fig. 3.10).

Já o desdobramento dos ramos $\left[ \pm\right.$ na Eq. (3.17)] para $T_{L}=5 \mathrm{~K} \mathrm{e}$ $B=0.5 \mathrm{~T}$ é de $\sim 0.8 \mathrm{ps}$, bem menor que o valor experimental que é de $\sim 8 \mathrm{ps}$. Um dos principais resultados do trabalho da Ref. [39] é corrigir esta diferença, o que feito considerando os fatores de Fermi e a sua dependência com o campo magnético.

Fatores de Fermi $\mathrm{O}$ fator de fermi $1-f_{m}^{\downarrow}$, Eq. (3.10), representa a probabilidade de haver espaço de fase disponível na banda destino (minoritária) no 


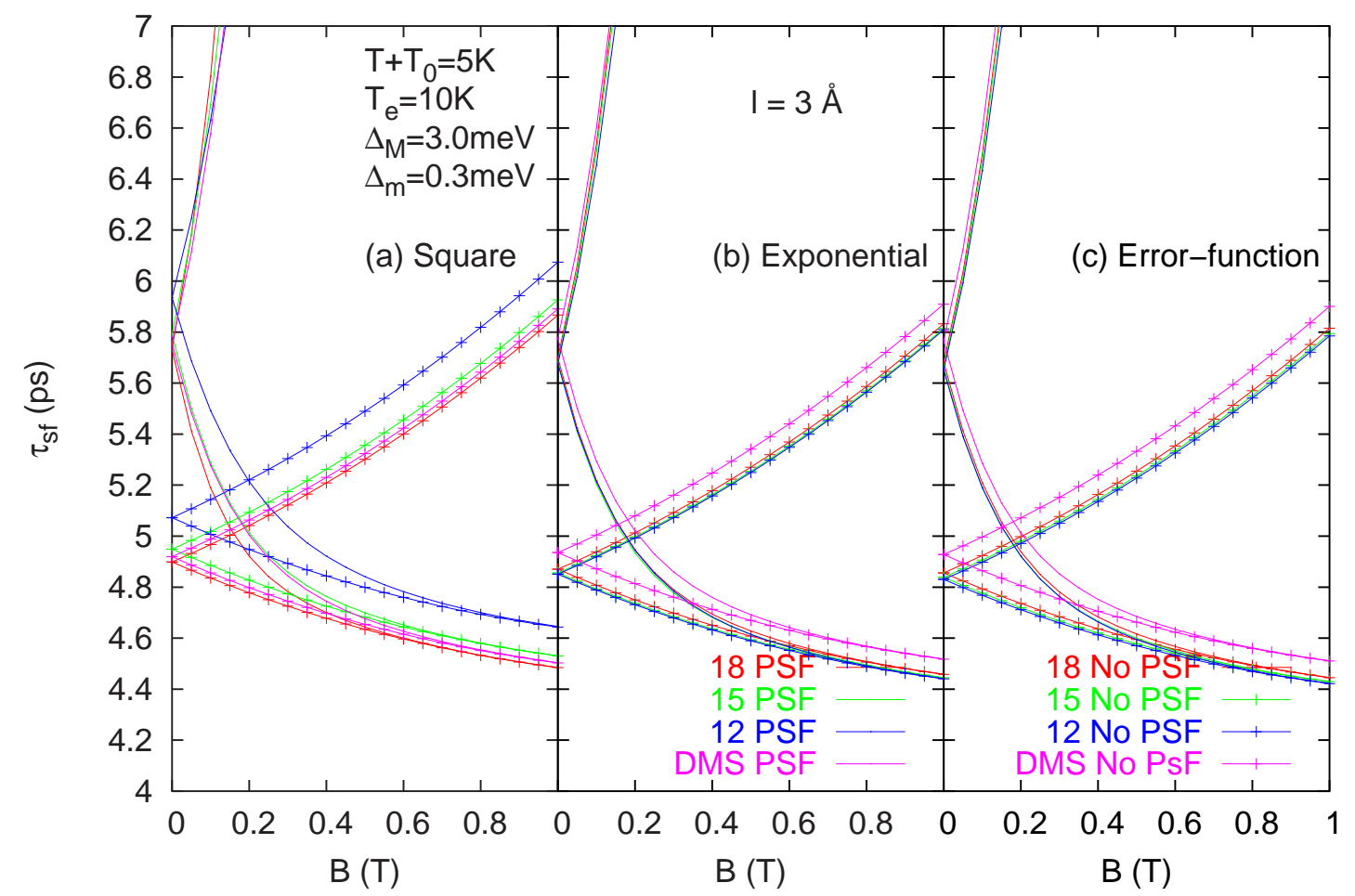

Figura 3.13: Tempo de espalhamento de spin teórico em função do campo magnético e usando concentração de Mn nominal. Os três painéis comparam $\tau_{\mathrm{sf}}$ para três tipos de perfil de concentração. Neste caso, o efeito da diluição é pequeno e a magnitude de $\tau_{s f}$ é aproximadamente a metade dos valores experimentais. A inclusão da estatística de Fermi (PSF) acentua o desdobramento entre os dois ramos, mas com um desdobramento muito maior que o experimentalmente medido.

mesmo patamar energia que do elétron na banda de origem (majoritária) - fator de preenchimento de fase (PSF - phase space filling). Na Ref. [39], os autores mostraram que a inclusão dos fatores de Fermi é essencial para descrever a dependência dos tempos de espalhamento com o campo magnético. Adicionalmente, esta dependência é geral, independente de um mecanismo particular de espalhamento.

$\mathrm{Na}$ Ref. [39], os autores apresentaram o tempo de espalhamento de spin $\tau_{s f}\left(\varepsilon_{k_{\|}}\right)$separadamente para vários valores de $\varepsilon_{k_{\|}}$, Eq. (3.7). Ou seja, $\tau_{s f}$ calculado desta forma não considera os fatores de ocupação na banda de origem, mas apenas a disponibilidade de espaço de fase na banda de destino.

Assim, aqui eu procedo um passo além do que foi calculado em [39], e calculo um tempo de espalhamento médio considerando conjuntamente as ocupações das bandas origem e destino. Esta média é dada simplesmente por

$$
\frac{1}{\tau_{s f}^{\uparrow \downarrow}}=F(B) \Gamma\left(N_{b}, \ell\right) \frac{\int_{0}^{\infty} f_{M}^{\uparrow}\left(\varepsilon_{k_{\|}}\right)\left[1-f_{m}^{\downarrow}\left(\varepsilon_{k_{\|}}\right)\right] \mathrm{d} \varepsilon_{k_{\|}}}{\int_{0}^{\infty} f_{M}^{\uparrow}\left(\varepsilon_{k_{\|}}\right) \mathrm{d} \varepsilon_{k_{\|}}},
$$




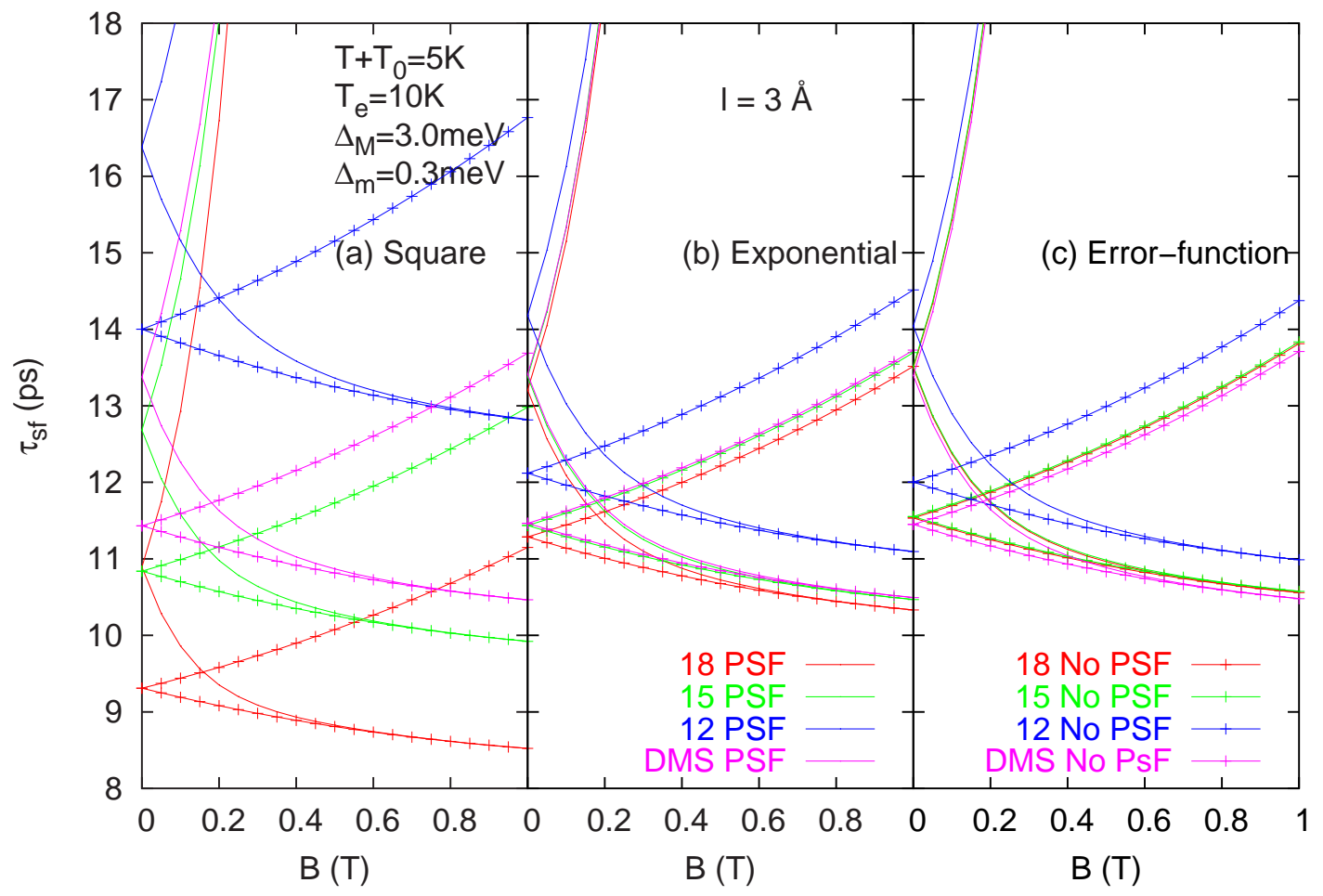

Figura 3.14: Tempo de espalhamento de spin teórico em função do campo magnético e usando concentração de Mn efetiva. Os valores de $\tau_{\text {sf }}$ são aproximadamente o dobro que no caso nominal, mas comparáveis com os valores experimentais. Para o caso de perfil ideal (a), $\tau_{s f}$ para diferentes configurações são bem distintos. A inclusão de diluição [(b) e (c)] diminui estas diferenças, restringindo os valores de $\tau_{s f}$ para uma faixa mais reduzida $(\mathrm{a} B=0)$, como nos dados experimentais. Os fatores de Fermi aumentam o desdobramento entre os dois ramos (PSF); sem este fatores (no PSF), o desdobramento dos ramos é aproximadamente 10 vezes menor que o caso experimental.

sendo

$$
f_{M}^{\uparrow}\left(\varepsilon_{k_{\|}}\right)=\left\{\exp \left[\left(\varepsilon_{1}^{\uparrow}+\varepsilon_{k_{\|}}-\mu_{M}^{\uparrow}\right) / k_{B} T_{e}\right]+1\right\}^{-1}
$$

Nos parágrafos seguintes eu apresento os resultados para os tempos de espalhamento induzidos pela interação de troca $s-p$.

\subsubsection{Resultados para $\tau_{s f}$}

Para investigar o efeito dos perfis não-ideais e aglomeração Mn-Mn nos tempos de espalhamento de spin induzidos por troca $s-d$, calculei $\tau_{s f}$ para várias configurações. As Figs. 3.13 e 3.14 mostram a dependência com o campo magnético de $\tau_{s f}$ para as transições $\uparrow \downarrow$ (ramo inferior) e $\downarrow \uparrow$ (ramo superior) para os casos usando concentrações nominais e efetivas, respectivamente. As duas figuras apresentam os resultados para $\tau_{s f}$ médio, Eq. (3.18), para os casos com (PSF) e sem (No PSF) os fatores de Fermi. Confirmando os resultados da Ref. [39], que calculou 
$\tau_{s f}\left(\varepsilon_{k_{\|}}\right)$, e não a média sobre todos os $\varepsilon_{k_{\|}}$'s como eu faço aqui, a não inclusão dos fatores de Fermi retorna um desdobramento entre os ramos $\uparrow \downarrow$ e $\downarrow \uparrow$ muito menor que o experimentalmente medido, Fig. 3.10. Por outro lado, a inclusão de PSF aumenta o desdobramento entre os ramos.

Caso de concentração nominal Os tempos característicos para o caso de concentração nominal (Fig. 3.13) ficaram menores que no caso de concentração efetiva (Fig. 3.14). Isto ocorre, como já discutido, pois a aglomeração antiferromagnética do segundo caso diminui a quantidade de spins de Mn ativos no processo de espalhamento, aumentando o tempo entre um processo de espalhamento e outro. No caso nominal, as diferenças entre as configurações com $N_{b}$ 's diferentes é pequena pois todas as impurezas de Mn contribuem para o espalhamento, (quase-)independente de como elas estão distribuídas no DMQW. A diferença entre os vários casos vem da superposição dos planos de Mn com a função de onda (fator $\Gamma$ ilustrado na Fig. 3.12), que varia pouco neste caso. A inclusão de diluição [painéis (b) e (c) da Fig. 3.13)] diminui mais ainda estas diferenças.

Caso de concentração efetiva A Fig. 3.14 mostra os resultados para $\tau_{s f}$ para o caso utilizando concentrações efetivas. Neste caso, apenas uma fração das impurezas de Mn contribui para o espalhamento de spin mediado pela interação de troca s-d (aglomeração antiferromagnética). Portanto, (i) os tempos são maiores que o caso nominal, e (ii) o efeito de diluição, seja distribuindo o Mn em várias barreiras, ou seja devido a segregação ou interdifusão, é importante para a magnitude de $\tau_{s f}$. O painel (a) da Fig. 3.14 reproduz os resultados da Ref. [39] para o caso de perfis ideais (quadrados). Note que, neste caso, os casos para $N_{b}$ 's diferentes são bem distintos, independente se considera-se PSF ou não.

Já os painéis (b) e (c) contêm os resultados que consideram interdifusão e segregação, respectivamente, no perfil de concentração do Mn. Para os casos com muitas barreiras, e.g. $N_{b}=18$, a diluição aumenta a superposição entre barreiras vizinhas, aumentando a aglomeração antiferromagnética e, portanto, aumentando o tempo de espalhamento (menos íons de Mn participando do processo). Para um número de barreiras menor, e.g. $N_{b}=12$, o efeito da diluição é diminuir a aglomeração antiferromagnética, aumentando, então, a quantidade de Mn ativa no espalhamento, e diminuindo $\tau_{s f}$. Assim, quando comparado com o caso ideal (barreiras quadradas) do painel (a), as curvas de $\tau_{s f}$ tendem a ficar mais próximas entre si quando se considera diluição, (b) e (c). Isto está de acordo com o fato experimental que a $B=0$ os tempos de espalhamento de spin são bem próximos para todas as configurações [RI] (veja a Fig. 3.10). 


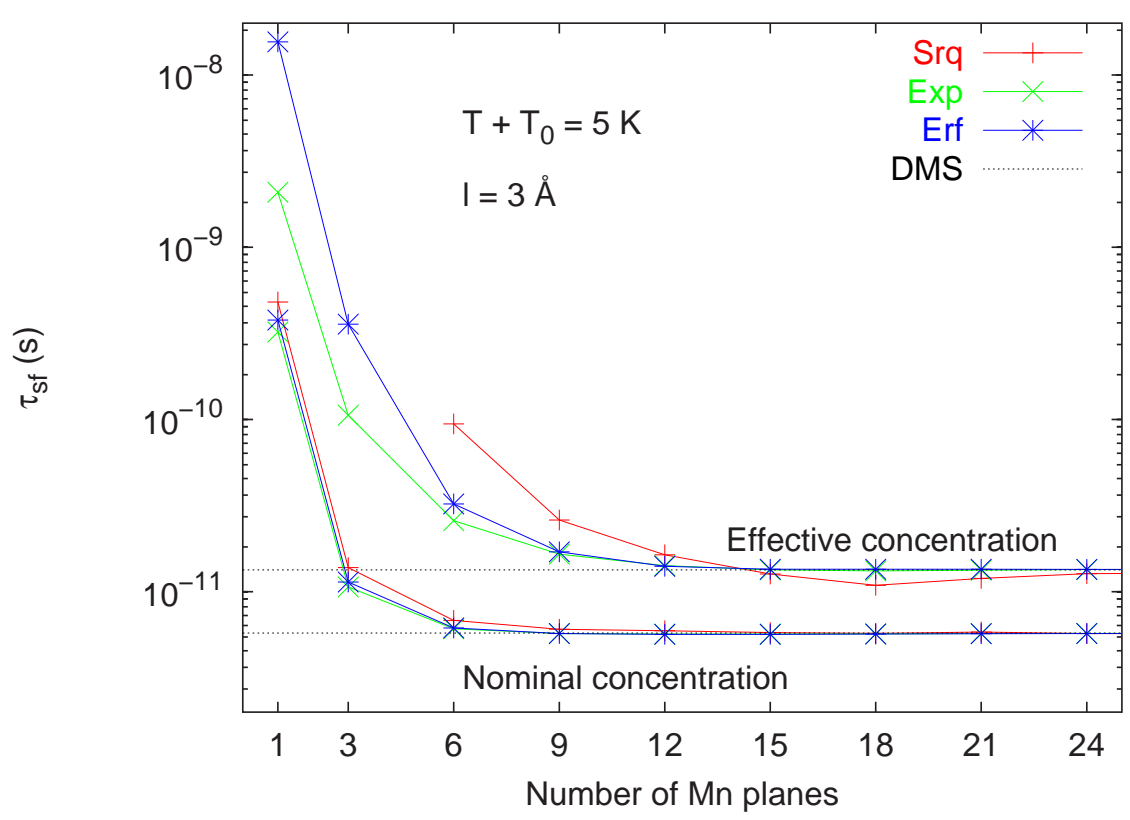

Figura 3.15: Tempo de espalhamento de spin em função do número de barreiras magnéticas para os casos de concentração nominal e efetiva. $\tau_{\text {sf }}$ descrito no modelo aumenta em até três ordens de magnitude no para os casos com poucas barreiras magnéticas. Nestes casos outros mecanismos de espalhamento seriam mais relevantes fazendo que os tempos de espalhamento observados nunca fossem tão grandes. Para cada configuração com $N_{b}$ barreiras $x_{p}=3 / N_{b}$.

Como foi dito no início desta seção, é importante lembrar que o mecanismo de espalhamento por troca $s-d$ não é necessariamente dominante em DMHs. Amostras não-magnéticas têm tempos de espalhamento de spin comparáveis àquelas com $\mathrm{Mn}$, apesar que apenas as magnéticas têm os dois ramos distintos para os espalhamentos $\uparrow \downarrow$ e $\downarrow \uparrow[39]$. A interação de troca s-d é importante para o desdobramento de spin gigante entre as bandas, afetando sim os tempos de espalhamento, mas indiretamente, via os fatores de Fermi. Mesmo se o mecanismo do espalhamento não for por via troca $s-d$, os fatores de Fermi ainda estarão presentes e devem ser relevantes [39].

De fato, como ilustrado na Fig. 3.15, os valores de $\tau_{s f}$ devido a troca s-d para para $N_{b} \leq 12$ são muito altos. Eles certamente são maiores que os tempos de espalhamento de spin devido a outros mecanismos, uma vez que os resultados experimentais de RI mostram que $\tau_{s f}$ para $N_{b}=3$ e 6 são comparáveis ao caso $N_{b}=24$.

\subsection{Discussões}

Neste capítulo eu investiguei o efeito da aglomeração antiferromagnética e da diluição das impurezas magnéticas nas propriedade mangetoópticas de DMHs. 
Eu mostrei que a inclusão destes efeitos melhoram os resultados teóricos para o desdobramento de spin e para tempos de espalhamento de spin, aproximando dos resultados experimentais disponíveis.

Esta parte do trabalho foi uma complementação da investigação da Ref. [39]. Naquele trabalho, os autores estudaram o desdobramento de spin e o espalhamento de spin induzidos pela interação de troca $s p$-d utilizando perfis quadrados (ideais) para a distribuição das impurezas magnéticas, e concentrações efetivas usando o modelo de singletos da teoria de estatística de aglomerados. O acordo da Ref. [39] com os dados experimentais foi qualitativo. Nesta parte da tese eu mostrei que a inclusão de efeitos de diluição do Mn, seja por segregação ou por interdifusão, mais o modelo de estatística de aglomerados que, além dos singletos, também considera tripletos abertos e fechados, permite obter um acordo quantitativo com os dados experimentais para o desdobramento de spin.

No que se refere aos tempos de espalhamento de spin, a inclusão dos efeitos de diluição preservou o resultado do trabalho anterior [39] no aspecto que os fatores de Fermi, que são gerais, possibilitam aumentar o desdobramento das curvas de tempo de espalhamento. Nestes estudos, o espalhamento via a interação de troca $s$-d é usado como um mecanismo modelo, relativamente simples, que permite investigar o efeito da inclusão dos fatores de preenchimento do espaço de fase. Se por um lado os valores de $\tau_{s f}$ para $N_{b} \geq 12$ estão muito próximos dos valores experimentais, a Fig. 3.15 mostra que os resultados para um número menor de barreiras diverge dos resultados experimentais em até três ordens de magnitude. Isto reforça a ideia que troca s-d não é mesmo o principal mecanismo de espalhamento de spin nestes sistemas.

Voltando para os resultados para o desdobramento de spin, se por um lado Awschalom e Samarth [60] dizem que há "evidências circunstanciais" sugerindo que os efeitos de interdifusão e segregação nestes sistemas são limitados a escalas de uma monocamada (veja Sec. 2.1), os resultados aqui apresentados também permitem inferências à respeito da configuração microscópica dos arranjos de Mn. Baseado nos modelos para aglomeração e diluição usados nesta tese, é possível estimar que os comprimentos característicos de diluição são da ordem de uma a duas monocamadas. Entretanto, com os dados disponíveis, não é possível especificar se o mecanismo relevante na diluição é de segregação ou interdifusão. Sobre este aspecto, os experimentos usando barreiras normais e invertidas [59, 6166], que eu mencionei na Sec. 2.1, são mais conclusivos e sugerem que segregação é o mecanismo mais relevante.

Em bulk, para $x \lesssim 0.05$ os diversos modelos da estatística de aglomerados são equivalentes, enquanto a parametrização de Gaj et al. [59], Eq. 2.2, pode ser usada para toda a faixa de valores (o modelo de aglomerado incluindo a 
correção de ordem maior aproxima bem deste resultado até $x \lesssim 0.15)$. Já para os casos de interfaces e (mono)camadas, não há uma análise experimental extensa, semelhante a existente para bulk, que sirva de parâmetro de comparação para os modelos teóricos, seja usando estatística de aglomerados ou o modelo $x_{N N}$. Assim, para valores de $x>0.1$, apenas a comparação de resultados teóricos com dados experimentais, para grandezas que dependem tanto da aglomeração antiferromagnética quanto da diluição, pode dizer se o modelo adotado foi adequado ou não. De fato, foi isto que fiz para o caso de desdobramento de spin: o efeito de diluição, seja por segregação ou interdifusão, mais o modelo de aglomerados com singletos e tripletos, permitiu ajustar melhor os dados experimentais.

É importante lembrar, porém, que devido a limitação dos modelos para o efeito de aglomeração para as amostras com alto $x$, o comprimento característico $\ell$ e a concentração nominal $x_{p}$ são, em princípio, apenas parâmetros que regulam a magnitude de $\Delta E$. Para se fazer uma identificação entre os valores usados para $\ell$ e $x_{p}$ e os valores microscópicos reais seria necessário verificar estas grandezas independentemente e, de preferência, com métodos diretos. Por exemplo, a técnica de microscopia de tunelamento de seção transversal (X-STM - cross-sectional scanning tunneling microscopy) [84-88] possibilita "visualizar" com precisão atómica o perfil estrutural de uma heteroestrutura . Nesta técnica, a amostra é seccionada de forma que a ponta do STM percorre a direção de crescimento da amostra. Assim, é possível traçar o perfil de concentração, indicando inclusive a quantidade de impurezas que de fato foi incorporada na amostra. Em especial, eu menciono as Refs. [87, 88], que investigaram super-redes digitais de InGaAlAs usando X-STM. Os resultados indicaram tanto a existência de interdifusão, quanto de segregação. A mesma técnica poderia ser utilizada para investigar o perfil de concentração de Mn em DMHs do grupo II-VI.

No restante deste trabalho eu utilizo o perfil de concentração do tipo segregação e o modelo de estatística de aglomerados com singletos e tripletos $\left(P_{1}+P_{3}\right)$, a menos que indicado o contrário. Para cada caso é determinado qual comprimento característico e concentração de Mn melhor ajustam os cálculos teóricos aos respectivos dados experimentais. Desta forma, agora eu retiro o enfoque das impurezas magnéticas e passo para a física de gases de elétrons bidimensionais que são confinados em DMHs. O papel do Mn e da interação de troca $s$-d é, via o desdobramento de spin gigante, polarizar este gás de elétrons e alinhar níveis de Landau de spins opostos. Sob estas condições surgem os efeitos não triviais que são analisados nos próximos capítulos. 


\section{Parte II}

\section{Magnetotransporte e \\ Ferromagnetismo Itinerante}





\section{Capítulo 4}

\section{Gás de Elétrons Bidimensional e Quantização de Landau}

\section{Conteúdo}

A introdução de dopagem em heteroestruturas digitais magnéticas resulta na formação dos chamados gases de elétrons bidimensionais. $\mathrm{Na}$ presença de um campo magnético intenso, a energia cinética é quantizada, formando os níveis de Landau. Efeitos quânticos oriundos das quantizações espacial e orbital refletem em diversas grandezas, em particular, no magnetotransporte, que exibe o efeito Hall quântico. Neste capítulo eu apresento a formulação que eu utilizo nesta tese para calcular a estrutura eletrônica e as propriedades de magnetotransporte deste tipo de sistema. O contexto teórico baseia-se na formulação autoconsistente de Kohn-Sham para a teoria do funcional da densidade dependente de spin. Eu utilizo esta teoria na aproximação da massa efetiva e função envelope.

4.1 Introdução . . . . . . . . . . . . . . . . . . 74

4.2 Estrutura eletrônica: DFT/LSDA . . . . . . . . . . . . . 77

4.3 Campo magnético e quantização de Landau . . . . . . . . . . . . 90

4.4 Magnetotransporte no regime quântico . . . . . . . . . . . . . . . 99

4.5 Discussões e resumo da formulação . . . . . . . . . . . . . 106 


\subsection{Introdução}

Nos capítulos anteriores eu investiguei as propriedades magnetoópticas de DMHs, e a sua conexão com os efeitos de aglomeração e diluição do Mn nestas heteroestruturas. Agora eu mudo o enfoque para propriedades de magnetotransporte de DMHs com dopagem por modulação do tipo $n$. Estas heteroestruturas são semelhantes ao poço quântico digital magnético ilustrado na Fig. 4.1. Eu analiso experimentos recentes usando DMHs do grupo II-VI [21, 37, 38] realizados no regime de efeito Hall quântico inteiro (IQHE). O desdobramento de spin gigante devido, a interação de troca s-d, permite alinhar os níveis eletrônicos (níveis de Landau, LLs) tal que novos efeitos surgem nas medidas de magnetotransporte. A Ref. [37] relata o aparecimento de um pico anômalo nas medidas de resistividade longitudinal $\rho_{x x}$, e o deslocamento da curva de resistividade para campos magnéticos mais baixos à medida que a temperatura aumenta. Já na Ref. [21], além de também ocorrer um pico anômalo em $\rho_{x x}$, este pico apresentou um ciclo de histerese à medida que o campo magnético aumentava ou diminuia. Este resultado é uma forte evidência da existência de um regime de ferromagnetismo itinerante (dos elétrons de condução) denominado de ferromagnetismo de efeito

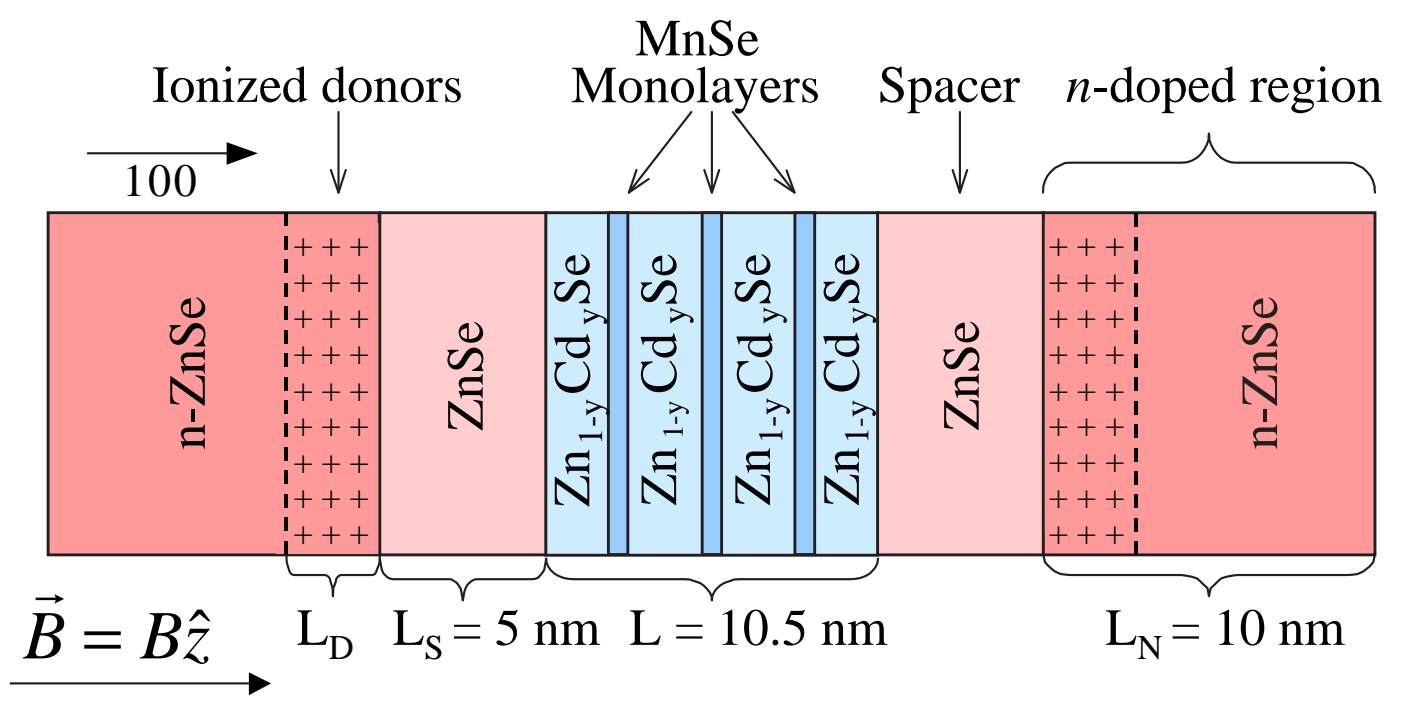

Figura 4.1: Esquema de um poço quântico digital magnético dopado por modulação. O poço quântico situa-se no centro da heteroestrutura crescida, e.g., na direção (100). Nele são inseridas (mono)camadas de MnSe regularmente espaçadas. As regiões das barreiras são compostas por espaçadores e regiões com alta dopagem. As impurezas doadoras fornecem os elétrons que são capturados pelos níveis ligados do poço. Na presença de um campo magnético, os momentos magnéticos localizados dos átomos de Mn se alinham e interagem fortemente com os elétrons confinados na região do poço, conferindo ao sistema uma resposta paramagnética bastante intensa (interação de troca $s p-d$ ). 
Hall (QHF - quantum Hall ferromagnetism) [89].

Gás de elétrons bidimensional À partir deste capítulo da tese, o principal personagem passa ser o gás de elétrons que se forma na região de confinamento de DMHs como a da Fig. 4.1 - semelhantes àquelas que investiguei no Cap. 3, mas com altos níveis de dopagem. Quando o movimento dos portadores é restrito a regiões da ordem de seu comprimento de onda de de-Broglie $(\lambda=2 \pi / k=$ $h / \sqrt{2 m E} \sim 122 \AA$ para $m / m_{0}=0.1$ e $E=0.1 \mathrm{eV}$ ), a dinâmica passa a ser regida pela mecânica quântica. O movimento é "congelado" na direção de confinamento, i.e., a separação entre os níveis confinados é bem maior que o alargamento térmico $\left(\sim k_{B} T=0.086 \mathrm{meV} \mathrm{K}^{-1}\right)$ e o alargamento devido a colisões $(\Gamma \sim 0.7 \mathrm{meV}) .{ }^{1}$ Assim, o movimento só é possível no plano transversal, formando o chamado gás de elétrons bidimensional (2DEG).

As heteroestruturas semicondutoras aqui investigadas fazem parte desta classe de sistemas capazes de confinar 2DEGs. A realização prática destes gases quânticos levou à descoberta das contrapartidas quânticas do efeito Hall, a saber, o inteiro [90] e o fracionário [91, 92]. No regime destes efeitos, que por si próprios envolvem uma física muito rica, surgem outros tão interessantes quanto, como, por exemplo, o ferromagnetismo itinerante de efeito Hall [20, 89]. Os Capítulos 5 e 6 abordam justamente fenômenos de magnetotransporte e ferromagnetismo itinerante no regime do efeito Hall quântico inteiro. Nestes casos, o sistema é submetido a campos magnéticos intensos, perpendiculares à direção de crescimento da amostra.

Dopagem por modulação As DMHs utilizadas nos experimentos de magnetotransporte que eu investigo possuem impurezas doadoras do tipo $n$. Estas são implantadas nas regiões das barreiras, ${ }^{2}$ em geral separadas do poço quântico por regiões espaçadoras intrínsecas (veja a Fig. 4.1). Estas regiões $n$-dopadas funcionam como um reservatório de elétrons que, quando capturados na região de confinamento do DMQW, formam um 2DEG.

Como estes sistemas dopados são utilizados em medidas de magnetotransporte, é desejável ter uma grande concentração de portadores com a maior mobilidade possível. Por outro lado, os dopantes são impurezas espalhadoras que acabam por limitar a mobilidade. Para contornar esta dificuldade, a técnica de

\footnotetext{
${ }^{1} \Gamma \sim \hbar / \tau$, e $\tau=m \mu / e$, sendo $m / m_{0} \sim 0.16$ e $\mu \sim \mathrm{m}^{2} / \mathrm{Vs}$ valores típicos para elétrons.

${ }^{2}$ Dopantes do tipo $n$ típicos nestas estruturas do grupo II-VI são elementos que possuem 7 elétrons na última camada. Nas DMHs baseadas em ZnSe investigadas nesta tese (Cap. 5), o dopante é o Cloro [37], cuja configuração eletrônica é [Ne] $3 s^{2} 3 p^{5}$, e que entra na sub-rede de anions substituindo o Selênio ([Ar] $\left.4 s^{2} 3 d^{10} 4 p^{4}\right)$. Já nos sistemas de CdTe (Cap. 6), o dopante é o Iodo [21] ([Kr] $\left.5 s^{2} 4 d^{10} 5 p^{5}\right)$, que substitui o Telúrio ([Kr] $\left.5 s^{2} 4 d^{10} 5 p^{4}\right)$. O elétron adicional da camada $p$ confere uma dopagem do tipo $n$.
} 

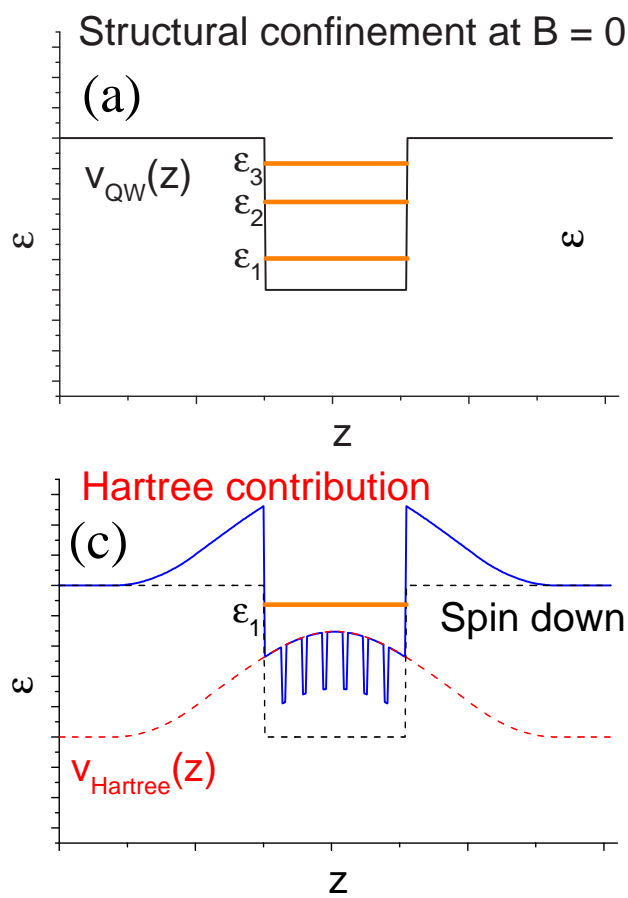

sp-d exchange interaction $B \neq 0$

(b)
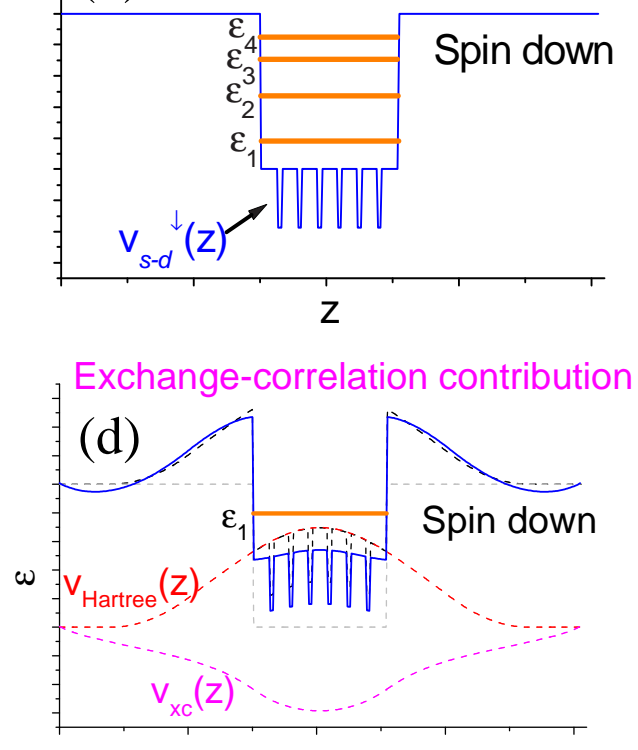

Z

Figura 4.2: Descrição qualitativa dos principais componentes do potencial de um poço quântico digital magnético, semelhante ao ilustrado na Fig. 4.1. Em cada interface $\mathrm{ZnSe} /(\mathrm{Zn}, \mathrm{Cd}) \mathrm{Se}$ há uma descontinuidade do potencial, formando um poço quântico com uma série de níveis ligados (a). Na presença de um campo magnético, a interação de troca $s p$-d contribui com um perfil de potencial dependente de spin que segue o perfil de concentração do Mn (b). Os elétrons confinados dentro do poço quântico exercem uma repulsão Coulombiana entre si extremamente forte. O potencial de Hartree correspondente deve ser somado aos potenciais estrutural e magnético (c). Por ser um gás de elétrons quântico, além do termo de Hartree, há também a contribuição de troca e correlação, que atenua o termo de Hartree (d).

dopagem por modulação [93] é utilizada. Nela, a dopagem é feita numa região diferente daquela que vai capturar e confinar as cargas. Portanto, há uma separação espacial entre a densidade de portadores ativa no transporte e as impurezas espalhadoras. Isto permite mobilidades elevadas $\left(\sim 10^{4} \mathrm{~cm}^{2} / \mathrm{Vs}\right)$ em sistemas altamente dopados $\left(\sim 10^{19} \mathrm{~cm}^{-3}\right)$.

Esta separação espacial da densidade de portadores e suas impurezas ionizadas resulta em campos elétricos internos. Tanto as regiões ionizadas (positivas) contribuem com um potencial de Coulomb atrativo, quanto as regiões com excesso de elétrons (negativas) contribuem com um potencial repulsivo. Estes potencias, que seguem o perfil de densidade de carga, são somados ao potencial estrutural do sistema, resultando o potencial típico de heteroestruturas com dopagem por modulação.

Na Fig. 4.2 eu ilustro como cada ingrediente do sistema contribui 
para o perfil de potencial total. Os painéis (a) e (b) mostram os perfis de potencial estrutural e de interação de troca $s-d$, como aqueles utilizados nos capítulos anteriores [Eq. 3.2]. A separação de cargas, devido à dopagem por modulação, gera campos elétricos internos, cujo potencial eletrostático correspondente está ilustrado no painel (c). Como o gás de elétrons confinado na região do poço é um sistema quântico, há também contribuições de troca e correlação para o potencial total (d), cujo efeito é atenuar o potencial de Hartree.

Organização do capítulo Neste capítulo eu complemento o modelo para DMHs apresentado no Cap. 1 incluindo dois novos ingredientes. Eu passo a considerar na formulação do problema a interação de Coulomb e a quantização de Landau do gás de elétrons confinado nas DMHs. O confinamento de uma alta concentração de elétrons acarreta uma forte repulsão de Coulomb. Aqui eu trato este sistema interagente usando a formulação autoconsistente de Kohn-Sham [41] da teoria do funcional da densidade (DFT - density-functional theory) [40]. Na Seção 4.2 eu faço um resumo dos aspectos da DFT que são importantes neste trabalho. Em seguida, na Seção 4.3, eu considero o efeito do campo magnético neste gás de elétrons, que resulta na chamada quantização de Landau. Para analisar os resultados experimentais de magnetotransporte investigados nos capítulos $5 \mathrm{e}$ 6 seguintes, eu apresento na Seção 4.4 o modelo usado para simular as magnetorresistências longitudinal $\rho_{x x}$ (efeito Shubnikov-de Haas, SdH) e transversal $\rho_{x y}$ (IQHE). Adiantando os resultados, utilizando a formulação que é apresentada no restante deste capítulo, foi possível reproduzir quantitativamente as principais características presentes nos dados experimentais, inclusive o efeito de QHF.

\subsection{Estrutura eletrônica: DFT/LSDA}

Como nestes sistemas dopados há uma enorme quantidade de elétrons $\left(\sim 10^{11} \mathrm{~cm}^{-2}\right)$ confinada numa região relativamente estreita $(L \sim 100 \AA)$, ocorre forte repulsão de Coulomb que deve ser levada em consideração no cálculo da estrutura eletrônica do sistema e das demais propriedades. O 2DEG é um sistema quântico, com as funções de onda de seus constituintes estendidas na escala do poço quântico e sobrepostas entre si. Neste regime, além da contribuição "clássica" de Hartree, há também a parte quântica da interação de Coulomb, chamada de troca-correlação (XC). Tratar estas contribuições é um problema complicado de muitos corpos. Aqui eu utilizo a formulação de Kohn-Sham (KS) da teoria do funcional da densidade dependente de spin [40, 41].

Nesta seção eu apresento a formulação de Kohn-Sham na forma apropriada para tratar as heteroestruturas magnéticas que eu analiso nos capítulos 
subsequentes. Os programas numéricos desenvolvidos implementam está técnica de cálculo de estrutura eletrônica para sistemas de muitos corpos.

\subsubsection{Formulação geral e sistema Kohn-Sham}

O Hamiltoniano de uma gás de elétrons interagente sujeito a um potencial externo $v(r)$ é escrito como

$$
\hat{H}=\hat{T}+\hat{U}+\hat{V}
$$

sendo

$$
\begin{aligned}
\hat{T} & =\frac{1}{2} \int \nabla \Psi^{*}(r) \cdot \nabla \Psi(r) \mathrm{d} r \\
\hat{U} & =\frac{1}{2} \int \frac{1}{\left|r-r^{\prime}\right|} \Psi^{*}(r) \Psi^{*}\left(r^{\prime}\right) \Psi\left(r^{\prime}\right) \Psi(r) \mathrm{d} r \mathrm{~d} r^{\prime}, \\
\hat{V} & =\int v(r) \Psi^{*}(r) \Psi(r) \mathrm{d} r
\end{aligned}
$$

as energias cinética, de Coulomb e do potencial externo, respectivamente. O operador de densidade é

$$
\mathrm{n}(r)=\Psi^{*}(r) \Psi(r)
$$

Na DFT, a energia do sistema é um funcional da densidade e é dada por $^{3}$

$$
E[n]=\langle\Psi[n]|\hat{T}+\hat{U}+\hat{V}| \Psi[n]\rangle=T[n]+U[n]+V[n] .
$$

Separando a parte não interagente $T_{s}[n]$ da energia cinética, e a parte de Hartree $U_{H}[n]$ da interação Coulomb, a energia total pode ser reescrita como

$$
E[n]=T_{s}[n]+U_{H}[n]+V[n]+E_{x c}[n] .
$$

O termo $E_{x c}[n]$ é a chamada energia de troca e correlação (XC - exchangecorrelation), e contem tanto a contribuição de troca da interação de Coulomb, quanto as contribuições de correlação da parte interagente da energia cinética e da interação Coulombiana.

Na formulação de Kohn-Sham (KS) da DFT [41], o problema de muitos corpos original é substituído por um problema fictício de uma única partícula sujeita a um potencial efetivo $v^{\prime}(r)$,

$$
\left[-\frac{\hbar^{2} \nabla^{2}}{2 m}+v^{\prime}(r)\right] \phi_{i}(r)=\varepsilon_{i} \phi_{i}(r) .
$$

\footnotetext{
${ }^{3}$ A notação $F[n]$ significa que a grandeza $F$ é funcional de $n$. Enquanto uma função faz um mapeamento entre números, $x \stackrel{f}{\rightarrow} y=f(x)$, um funcional $F$ faz um mapeamento entre funções e números, $f(x) \stackrel{F}{\rightarrow} y=F[f(x)]$, ou entre funções e funções, $f(x) \stackrel{F}{\rightarrow} g[f(x)](z)$, ou $g([f(x)] ; z)$.
} 
Os auto-estados $\phi_{i}(r)$ são orbitais monoeletrônicos e são chamados de orbitais Kohn-Sham. Analogamente, as auto-energias $\varepsilon_{i}$ são as energias de Kohn-Sham. Se o potencial efetivo $v^{\prime}(r)$ for apropriadamente escolhido tal que a densidade

$$
n^{\prime}(r)=\sum_{i=1}^{N}\left|\phi_{i}(r)\right|^{2}
$$

com $N$ o número total de partículas, minimize o funcional da energia $E\left[n^{\prime}\right]$, os teoremas da DFT garantem, então, que esta densidade, calculada à partir dos orbitais monoeletrônicos, é igual à densidade total do estado fundamental do sistema interagente de muitos corpos. Adicionalmente, a energia obtida à partir desta densidade é a energia do estado fundamental.

Para determinar o potencial efetivo $v^{\prime}(r)$ é efetuado um procedimento de minimização do funcional da energia com relação a densidade. Inicialmente, note que a energia cinética não interagente pode ser escrita utilizando as energias KS da Eq. (4.8),

$$
T_{s}\left[n^{\prime}\right]=\sum_{i}\left\langle\phi_{i}\left|-\frac{\hbar^{2} \nabla^{2}}{2 m}\right| \phi_{i}\right\rangle=\sum_{i} \varepsilon_{i}-\int v^{\prime}(r) n^{\prime}(r) \mathrm{d} r
$$

de forma que a energia total (4.7) fica

$$
E\left[n^{\prime}\right]=\sum_{i} \varepsilon_{i}-\int v^{\prime}(r) n^{\prime}(r) \mathrm{d} r+\frac{1}{2} \int \frac{n^{\prime}\left(r^{\prime}\right) n^{\prime}(r)}{\left|r-r^{\prime}\right|} \mathrm{d} r \mathrm{~d} r^{\prime}+\int v(r) n^{\prime}(r) \mathrm{d} r+E_{x c}\left[n^{\prime}\right] .
$$

Agora minimiza-se (4.11) com relação a $n^{\prime}$ para encontrar a condição que deve ser satisfeita para que a densidade $n^{\prime}$ corresponda à densidade do estado fundamental,

$$
\frac{\delta E\left[n^{\prime}\right]}{\delta n^{\prime}}=-v^{\prime}(r)+\underbrace{\int \frac{n^{\prime}\left(r^{\prime}\right)}{\left|r-r^{\prime}\right|} \mathrm{d} r^{\prime}}_{v_{h}\left(\left[n^{\prime}\right] ; r\right)}+v(r)+\underbrace{\frac{\delta E_{x c}\left[n^{\prime}\right]}{\delta n^{\prime}(r)}}_{v_{x c}\left(\left[n^{\prime}\right] ; r\right)}=0 .
$$

Assim, o potencial fictício é dado por

$$
v^{\prime}([n] ; r)=v(r)+v_{h}\left(\left[n^{\prime}(r)\right] ; r\right)+v_{x c}\left(\left[n^{\prime}(r)\right] ; r\right)
$$

no qual o primeiro termo é o próprio potencial externo, o segundo é o potencial de Hartree, e o terceiro é o potencial de troca e correlação.

As equações (4.8) e (4.13) formam um sistema autoconsistente pois estão acopladas pela densidade $n^{\prime}(r)$ [Eq. (4.9)]: ao mesmo tempo que para resolver a equação de Schrödinger é preciso conhecer o potencial efetivo, que é funcional da densidade, é preciso também conhecer as autofunções $\phi_{i}(\boldsymbol{r})$ para 
calcular a densidade $n(\boldsymbol{r})$. Assim, na prática o cálculo começa com uma condição inicial, e.g. $v_{h}=v_{x c}=0$, e continua resolvendo-se as equações (4.8) e (4.13) sucessivamente, usando o resultado de uma no cálculo da outra. ${ }^{4}$ Esta formulação autoconsistente é a forma mais utilizada da DFT em implementações práticas, que vão desde o cálculo de sistemas atómicos e moleculares até a física de estado sólido [94].

Substituindo (4.13) em (4.11) obtém-se a expressão para a energia total do sistema interagente em termos das energias monoeletrônicas de KohnSham,

$$
E\left[n^{\prime}\right]=\sum_{i} \varepsilon_{i}-\frac{1}{2} \int \frac{n^{\prime}\left(r^{\prime}\right) n^{\prime}(r)}{\left|r-r^{\prime}\right|} \mathrm{d} r \mathrm{~d} r^{\prime}-\int v_{x c}(r) n^{\prime}(r) \mathrm{d} r+E_{x c}\left[n^{\prime}\right]
$$

Desta forma, o problema original de muitos corpos ficou resumido a resolver a equação de Schrödinger de uma única partícula. Note, entretanto, que tanto a densidade $n^{\prime}(r)$ quanto as grandezas derivadas à partir dela, e.g. a energia $E\left[n^{\prime}\right]$, são soluções do sistema interagente de muitos corpos. Se o funcional $E_{x c}[n]$ para o sistema em questão fosse exatamente conhecido - e consequentemente o respectivo potencial $v_{x c}-$ a solução do problema seria exata. Entretanto, como este não é o caso, é necessário utilizar aproximações para a energia e para o potencial de troca-correlação.

Aproximações para $E_{x c}$ Uma das aproximações mais comuns é a aproximação da densidade local (LDA - local density approximation), na qual aproxima-se a energia de troca-correlação de cada elemento de volume do sistema com densidade $n(\boldsymbol{r})$ pelo valor conhecido desta energia para um gás de elétrons uniforme com a mesma densidade, ou seja,

$$
E_{x c}[n] \approx E_{x c}^{L D A}[n]=\int n(r) e_{x c}^{h o m}([n] ; r) \mathrm{d} r
$$

$\operatorname{com} e_{x c}^{h o m}([n] ; r)$ a energia de troca e correlação por partícula do gás de elétrons homogêneo. No caso da LDA, a contribuição de troca é conhecida exatamente

$$
\varepsilon_{x}^{h o m}[n]=-\frac{3}{4 \pi}\left(3 \pi^{2}\right)^{1 / 3} n^{1 / 3}
$$

\footnotetext{
${ }^{4}$ Para possibilitar a convergência do método, em geral o potencial autoconsistente a cada iteração é composto de uma fração do potencial da iteração anterior e outra fração do novo potencial, $v_{s c}(z)=\xi v_{s c}^{i-1}(z)+(1-\xi) v_{s c}^{i}(z)$. O valor de $\xi$ é em geral entre 0.5 e 0.9 . Durante as iterações uma ou mais grandezas são inspecionadas; quando elas param de variar o procedimento convergiu. Um teste de convergência é fazer $\xi=0$. Se a convergência estiver correta o resultado não deve mudar.
} 
em unidades atômicas [95]. Já para a correlação, normalmente utiliza-se valores obtidos em cálculos de Monte Carlo quântico [96]. Na prática, existem fórmulas paramétricas que reproduzem os dados conhecidos tanto para a energia quanto para o potencial XC [97].

\subsubsection{Extensão para sistemas magnéticos}

A introdução à DFT dos parágrafos anteriores limitou-se a sistemas não-magnéticos, para os quais os observáveis do sistema dependem da densidade $n(\mathbf{r})$. Quando considera-se a interação do campo magnético $\boldsymbol{B}$ com os spins eletrônicos, a energia total do sistema possui uma contribuição adicional dada por $-\int \boldsymbol{B}(\boldsymbol{r}) \cdot \mathbf{m}(\boldsymbol{r}) \mathrm{d} \boldsymbol{r}$, sendo $\mathbf{m}$ a magnetização do sistema eletrônico. Adicionalmente, nos sistemas investigados nesta tese, os spins também interagem com os momentos magnéticos localizados das impurezas de Mn, na chamada interação de troca sp-d. Como visto nos capítulos anteriores, esta interação pode ser incorporada ao problema via um potencial efetivo dependente de spin, Eq. (1.9).

A extensão da DFT para sistemas magnéticos consiste em considerar a magnetização do sistema $\mathbf{m}$ como uma variável fundamental, juntamente com a densidade $n$ [98]. Aqui eu considero o caso em que o campo magnético é uniforme na direção $\hat{z}, \boldsymbol{B}=B \hat{z}$, de forma que a magnetização é $\mathbf{m}=m \hat{z}$, com $m=\mu_{B}\left(n_{\uparrow}-n_{\downarrow}\right)$, em termos das densidades parciais de spin. Neste caso, ao invés de $\{n, m\}$ eu vou usar $\left\{n_{\uparrow}, n_{\downarrow}\right\}$ como variáveis fundamentais.

Considerando tanto a interação Zeeman, quanto um potencial dependente de spin $v_{\sigma_{z}}(\boldsymbol{r})$, a energia do potencial externo fica

$$
V\left[n_{\uparrow}, n_{\downarrow}\right]=\sum_{\sigma_{z}} \int\left[n_{\sigma_{z}} v_{\sigma_{z}}(r)+\mu_{B} n_{\sigma_{z}} \sigma_{z} B\right] \mathrm{d} r
$$

e a energia total fica

$$
\begin{aligned}
E\left[n_{\uparrow}, n_{\downarrow}\right] & =T\left[n_{\uparrow}, n_{\downarrow}\right]+U\left[n_{\uparrow}, n_{\downarrow}\right]+V\left[n_{\uparrow}, n_{\downarrow}\right] \\
& =T_{s}\left[n_{\uparrow}, n_{\downarrow}\right]+U_{H}\left[n_{\uparrow}, n_{\downarrow}\right]+V\left[n_{\uparrow}, n_{\downarrow}\right]+E_{x c}\left[n_{\uparrow}, n_{\downarrow}\right] .
\end{aligned}
$$

Da mesma forma que no caso não-magnético, é possível derivar um conjunto de equações autoconsistentes de partícula simples. Seguindo os mesmos passos da seção anterior, mas agora fazendo o procedimento de minimização da energia para as variáveis $\left\{n_{\uparrow}, n_{\downarrow}\right\}$, resulta uma equação de Schrödinger para cada componente de spin [98],

$$
\left[-\frac{\hbar^{2} \nabla^{2}}{2 m}+v_{\sigma_{z}}^{\prime}(r)\right] \phi_{i}^{\sigma_{z}}(r)=\varepsilon_{i}^{\sigma_{z}} \phi_{i}^{\sigma_{z}}(r), \quad \sigma_{z}= \pm 1
$$


na qual o potencial autoconsistente efetivo $v_{\sigma_{z}}^{\prime}(r)$ é

$$
v^{\prime}\left(\left[n_{\uparrow}, n_{\downarrow}\right] ; r\right)=\mu_{B} \sigma_{z} B+v^{\sigma_{z}}(r)+v_{h}\left(\left[n_{\uparrow}, n_{\downarrow}\right] ; r\right)+v_{x c}^{\sigma_{z}}\left(\left[n_{\uparrow}, n_{\downarrow}\right] ; r\right) .
$$

O potencial de troca e correlação é

$$
\begin{aligned}
v_{x c}^{\sigma_{z}}\left(\left[n_{\uparrow}, n_{\downarrow}\right] ; r\right) & =\frac{\delta E_{x c}\left[n_{\uparrow}, n_{\downarrow}\right]}{\delta n_{\sigma_{z}}}=\left.\frac{\partial\left[n(r) \varepsilon_{x c}\left(n_{\uparrow}, n_{\downarrow}\right)\right]}{\partial n_{\sigma_{z}}}\right|_{n_{\sigma_{z} \rightarrow n_{\sigma_{z}}}(r)} \\
& =\varepsilon_{x c}\left(n_{\uparrow}, n_{\downarrow}\right)+n(r) \frac{\partial \varepsilon_{x c}\left(n_{\uparrow}, n_{\downarrow}\right)}{\partial n_{\sigma_{z}}} .
\end{aligned}
$$

Similar ao caso não-magnético, aqui também são feitas aproximações para a energia (e para o potencial) de XC. A contrapartida da LDA para o caso dependente de spin é a aproximação da densidade de spin local (LSDA - local spin density approximation),

$$
E_{x c}\left[n_{\uparrow}, n_{\downarrow}\right] \approx E_{x c}^{L S D A}\left[n_{\uparrow}, n_{\downarrow}\right]=\int n(r) \varepsilon_{x c}^{h o m}\left(\left[n_{\uparrow}, n_{\downarrow}\right] ; r\right) \mathrm{d} r
$$

Em particular, neste trabalho eu utilizo a parametrização de Vosko, Wilk, e Nusair [99] (VWN) para $\varepsilon_{x c}^{\text {hom }}\left(\left[n_{\uparrow}, n_{\downarrow}\right] ; r\right) .^{5}$

A energia total em termos dos orbitais monoeletrônicos de Kohn-

Sham fica

$$
E\left[n_{\uparrow}, n_{\downarrow}\right]=\sum_{i, \sigma_{z}} \varepsilon_{i}^{\sigma_{z}}-\frac{1}{2} \int v_{h}(z) n(z) \mathrm{d} z-\sum_{\sigma_{z}} \int v_{x c}^{\sigma_{z}}(z) n_{\sigma_{z}}(z) \mathrm{d} z+E_{x c}\left[n_{\uparrow}, n_{\downarrow}\right] .
$$

Fator de escala de spin Aqui eu chamo a atenção para a dependência da contribuição de troca e correlação com a polarização do sistema eletrônico. As propriedades que eu menciono a seguir são fundamentais para explicar a contribuição de XC para aumentar o desdobramento de spin do gás de elétrons (Cap. 5), e para entender a estabilidade do estado ferromagnético eletrônico que surge nos experimentos analisados no Cap. 6.

A contribuição (negativa) para a energia devido a anti-simetrização da função de onda é a chamada energia de troca. Ela é máxima quando o gás de

\footnotetext{
${ }^{5}$ Eu tomei um cuidado especial com a utilização da DFT neste trabalho, em especial na escolha da parametrização para o potencial XC. Eu implementei diversas parametrizações existentes na literatura, não apenas da aproximação LSDA [100-103] mas também na aproximação de gradientes generalizada (GGA) [104-110]. A parametrização de Vosko, Wilk, e Nusair [99] é considerada atualmente uma das melhores para a LSDA e por isso eu a escolhi para efetuar os cálculos. Para os sistemas estudados nesta tese, a opção por GGAs não resulta uma melhora perceptível nos resultados. Já as LSDAs mais antigas, e.g. von Barth e Hedin [100] e Gunnarsson e Lundqvist [101], mostraram-se inadequadas uma vez que as simulações com estas parametrizações não reproduziram satisfatoriamente os resultados experimentais.
} 
elétrons está polarizado, i.e., todos os spins com a mesma orientação. Neste caso, o princípio de Pauli diz que a parte espacial da função de onda deve ser antisimétrica. Isto corresponde a uma maior separação espacial entre os máximos da densidade eletrônica, quando comparado com o caso não polarizado, e consequentemente, a uma energia de Coulomb menor. Desta forma, a energia do gás de elétrons polarizado é menor que no caso não-polarizado. Para o caso uniforme, a energia de troca por partícula de um sistema com polarização $\zeta=\left(n_{\uparrow}-n_{\downarrow}\right) / n=1$ é

$$
\varepsilon_{x}(n, \zeta=1)=2^{1 / 3} \varepsilon_{x}(n, \zeta=0),
$$

sendo que a energia para $\zeta=0$ é dada pela Eq. (4.16).

Para obter a dependência da energia de troca com a polarização, utiliza-se a seguinte propriedade de escala [111]

$$
E_{x}\left[n_{\uparrow}, n_{\downarrow}\right]=\frac{1}{2} E_{x}\left[2 n_{\uparrow}, 0\right]+\frac{1}{2} E_{x}\left[0,2 n_{\downarrow}\right] .
$$

Substituindo (4.24) em (4.25) obtém-se

$$
\varepsilon_{x}(n, \zeta)=\varepsilon_{x}(n, 0)+f(\zeta)\left[\varepsilon_{x}(n, 1)-\varepsilon_{x}(n, 0)\right],
$$

com

$$
f(\zeta)=\frac{(1+\zeta)^{4 / 3}+(1-\zeta)^{4 / 3}-2}{2\left(2^{1 / 3}-1\right)} .
$$

Enquanto a relação (4.26) é exata para troca (sistema uniforme), para correlação não existe uma relação similar. Uma aproximação comum é utilizar a mesma fórmula de troca para interpolar as energias de correlação, ou seja,

$$
\varepsilon_{c}(n, \zeta)=\varepsilon_{c}(n, 0)+f(\zeta)\left[\varepsilon_{c}(n, 1)-\varepsilon_{c}(n, 0)\right]
$$

chamada de interpolação de spin de von Barth e Hedin [100] (vBH). Assim, na LSDA, utiliza-se valores conhecidos para $\varepsilon_{c}(n, 0)$ e $\varepsilon_{c}(n, 1)$ para várias densidades e, via a Eq. (4.28), obtém-se a energia de correlação para uma polarização qualquer.

A interpolação de spin de vBH para a correlação foi amplamente utilizada na literatura por muitos anos. Atualmente a interpolação de spin de Vosko, Wilk, e Nusair [99] é considerada mais adequada,

$$
\varepsilon_{c}(n, \zeta)=\varepsilon_{c}(n, 0)+\alpha_{c}(n) \frac{f(\zeta)}{f^{\prime \prime}(0)}\left(1-\zeta^{4}\right)+\left[\varepsilon_{c}(n, 1)-\varepsilon_{c}(n, 0)\right] f(\zeta) \zeta^{4}
$$

na qual $\alpha_{c}(n)$ é tabulada para várias densidades, da mesma forma que $\varepsilon_{c}(n, 0)$ e $\varepsilon_{c}(n, 1)$. Esta é a interpolação de spin utilizada na parametrização da energia 


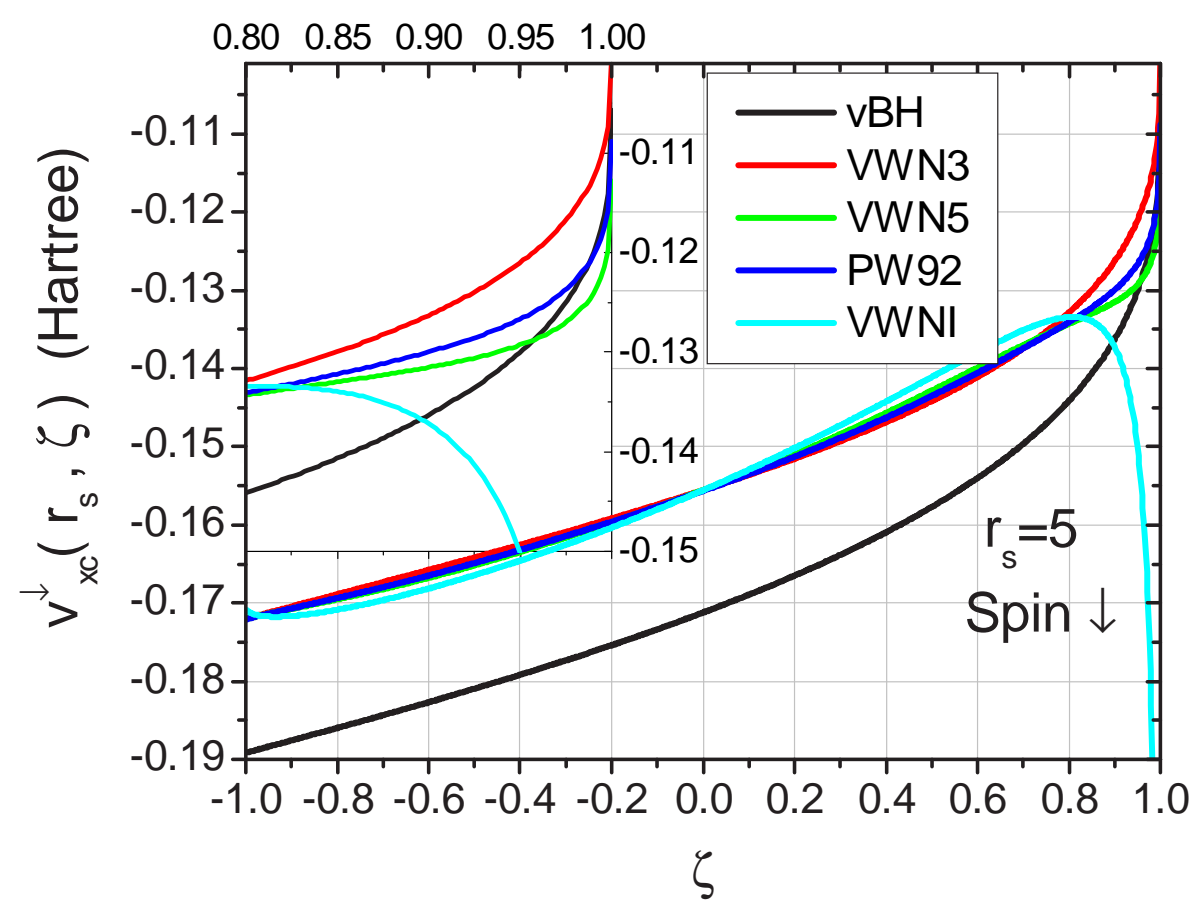

Figura 4.3: Potencial de troca e correlação em função da polarização $\zeta$ para um elétron com spin $|\downarrow\rangle$ num gás de elétrons com densidade $r_{s}=5$. São ilustradas várias parametrizações, tanto para a densidade, quanto para o spin (veja a Tabela 4.1). As duas primeiras indicadas na legenda usam a formula de interpolação de spin de vBH [100] e as duas seguintes usam a de VWN [99]. A última curva usa a parametrização de densidade de VWN com a interpolação de spin de Wang e Perdew [112] (VWNI); entretanto, o comportamento desta curva é completamente errado pois esta interpolação só é válida no limite de altas densidade. Quando $\zeta<0$, o elétron $|\downarrow\rangle$ é majoritário; já quando $\zeta>0$, este mesmo elétron é minoritário. Note que quanto mais negativa é a polarização, mais negativo é o potencial $v_{x c}^{\downarrow}$. Já quando o elétron é minoritário, $v_{x c}^{\downarrow}$ diminui (em módulo) abruptamente para $\zeta \rightarrow 1$. O inset é uma ampliação da região com $\zeta>0.8$. Ele mostra que as diferenças entre as diversas parametrizações são mais acentuadas para o potencial de XC do spin minoritário.

de correlação de VWN que eu uso nesta tese. Outras parametrizações também usam está fórmula de interpolação, como a PW92 [103]. A diferença entre estas parametrizações está nas fórmulas de interpolação dos valores conhecidos (Monte Carlo quântico [96]) para $\varepsilon_{c}(n, 0), \varepsilon_{c}(n, 1)$ e $\alpha_{c}(n)$ em função de $n$.

Uma vez escolhida a parametrização para a energia de XC, é preciso então calcular a potencial $v_{x c}^{\sigma_{z}}$ que é utilizado na equação de Kohn-Sham (4.19). Isto é feito utilizando a Eq. (4.21). Como mencionado anteriormente, a dependência deste potencial com a polarização do gás de elétrons é fundamental para entender alguns dos resultados dos próximos capítulos. Eu ilustro na Fig. 4.3 a dependência de $v_{x c}^{\downarrow}\left(r_{s}, \zeta\right)$ em função da polarização $\zeta$ para uma densidade fixa. As curvas são para spin $|\downarrow\rangle$; para o caso de spin $|\uparrow\rangle$, basta considerar o eixo da 
Tabela 4.1: Abreviaturas usadas na Fig. 4.3 para indicar a combinação de parametrização de carga e interpolação de spin. A parametrização de carga de VWN é combinada com três interpolações de spin.

\begin{tabular}{l|ccc}
\hline \hline Parametrização & \multicolumn{4}{|c}{ Interpolação de spin } \\
da densidade & vBH [100] & VWN [99] & WP [112] \\
\hline vBH [100] & vBH & - & - \\
VWN [99] & VWN3 & VWN5 & VWNI \\
PW92 [103] & - & PW92 & - \\
\hline \hline
\end{tabular}

polarização invertido, $\zeta \rightarrow-\zeta$. Há dois aspectos importantes a serem observados.

1. Para $\zeta \rightarrow 1$, o potencial $v_{x c}^{\downarrow}$ diminui (em módulo) abruptamente para um elétron $|\downarrow\rangle$. O mesmo ocorre para um elétron $|\uparrow\rangle$ quando $\zeta \rightarrow-1$. A consequência física direta desta propriedade é uma tendência favorecendo estados ferromagnéticos $(\zeta \rightarrow \pm 1)$ uma vez que o potencial XC local diverge para os elétrons minoritários [112].

2. Os elétrons majoritários ( $|\downarrow\rangle$ na figura) sentem um potencial mais negativo que os minoritários. Esta diferença reflete nas auto-energias do sistema, acarretando um desdobramento de spin devido troca e correlação.

A Fig. 4.3 também ilustra as diferenças existentes entre parametrizações para a energia de troca e correlação (e consequentemente para o respectivo potencial). Lá estão apresentadas três parametrizações para a densidade e três interpolações de spin. A Tabela 4.1 indica as combinações utilizadas. Note que as diferenças decorrentes de combinar parametrizações de carga e interpolações de spin ficam mais acentuadas à medida que o gás de elétrons fica polarizado. Isto mostra que, no regime de altas polarizações, a escolha adequada da aproximação para XC é fundamental. As parametrizações PW92 e VWN5 são consideradas as melhores LSDAs. Apenas por comparação eu também incluí o potencial XC de vBH; note que ele se destaca das demais para todo a faixa de polarizações. A interpolação de spin de Wang e Perdew [112] aqui apresentada também é apenas ilustrativa; esta interpolação é exata no limite de altas densidades, mas retorna uma dependência com $\zeta$ completamente errada fora do seu limite de validade, como ilustrado na Fig. 4.3.

\subsubsection{Generalização para temperatura finita}

Além da extensão da DFT para sistemas magnéticos, nesta tese eu utilizo uma extensão adicional: para temperaturas finitas [98, 113]. No estudo dos próximos 
capítulos abordando magnetotransporte em 2DEGs confinados em DMHs, vários dos efeitos observados experimentalmente, e que aqui eu calculo, estão relacionados com a variação da temperatura do sistema. Do ponto de vista prático, como eu mostrarei a seguir, a extensão para temperaturas finitas consiste em (a) utilizar a função de Fermi na densidade de estados do sistema Kohn-Sham, e (b), dependendo se o número de elétrons ou o potencial químico é constante, trabalhar ou com o potencial canônico ou o grão-canônico, respectivamente. Porém, é importante salientar que esta extensão é formalmente justificada dentro da DFT $[98,113]$.

Os casos estudados nos capítulos subsequentes são tais que a densidade total é constante quando se varia o campo magnético e a temperatura (o potencial químico varia). Assim, é conveniente trabalhar no ensemble canônico e a energia que é minimizada é a energia livre de Helmholtz. Aqui eu sigo os passos da Ref. [98], mas com a diferença que lá os autores trabalham no ensemble grãocanônico. Como nos casos já ilustrados nesta seção, o objetivo aqui é deduzir as equações de Kohn-Sham e obter a equação da energia total à partir das energias monoeletrônicas.

A energia livre de Helmholtz $A$ é obtida subtraindo-se a energia térmica da energia interna, ou seja,

$$
A[n]=T[n]+U[n]+V[n]-\tau S[n]
$$

sendo $\tau$ a temperatura ${ }^{6}$ e $S[n]$ é a entropia do sistema interagente de muitos corpos. Agora reescrevo $A[n]$ em termos das energias não interagentes $T_{s}$ e $U_{H}$, e da entropia não interagente $S_{s}[n]$ [114, pág. 54],

$$
S_{s}=-k_{B} \sum_{i} f_{i} \ln f_{i}+\left(1-f_{i}\right) \ln \left(1-f_{i}\right)
$$

$\operatorname{com} f\left(\varepsilon_{i}\right) \equiv f_{i}$ a função de Fermi

$$
f\left(\varepsilon_{i}\right)=\frac{1}{1+\exp \left[\left(\varepsilon_{i}-\mu\right) / k_{B} \tau\right]}
$$

e $\mu$ o potencial químico. A energia de Helmholtz fica

$$
A[n]=T_{s}[n]+U_{H}[n]+V[n]-\tau S_{s}[n]+F_{x c}[n],
$$

na qual $F_{x c}[n]$ é a energia de troca e correlação. Este termo é diferente de $E_{x c}[n]$ usado anteriormente pois contém a diferença entre a energia térmica interagente

\footnotetext{
${ }^{6}$ Nesta seção eu denoto a temperatura como $\tau$ para distinguir da energia cinética.
} 
e a não-interagente. Se o sistema interage apenas termicamente com o meio, a densidade de equilíbrio $n$ é tal que a energia livre de Helmholtz é mínima,

$$
\frac{\delta A[n]}{\delta n}=0=\frac{\delta\left(T_{s}[n]-\tau S_{s}[n]\right)}{\delta n}+v[n]+v_{H}[n]+f_{x c}[n]
$$

Agora considere a equação de Schrödinger de um sistema auxiliar, não interagente, sujeito ao potencial $v^{\prime}(r)$,

$$
\left[-\frac{\hbar^{2} \nabla^{2}}{2 m}+v^{\prime}(r)\right] \phi_{i}^{\prime}(r)=\varepsilon_{i}^{\prime} \phi_{i}^{\prime}(r) .
$$

A densidade total deste sistema na temperatura $\tau$ é

$$
n^{\prime}(r)=\sum_{i}\left|\phi_{i}^{\prime}(r)\right|^{2} f\left(\varepsilon_{i}^{\prime}\right)
$$

na qual a função de fermi especifica a ocupação dos níveis eletrônicos no caso de temperatura finita.

Como feito anteriormente, a questão é determinar qual é o potencial fictício $v^{\prime}(r)$ tal que a densidade $n^{\prime}(r)$ seja igual a densidade do sistema interagente de muitos corpos. Os passos são idênticos às passagens de (4.10) para (4.12), resultando no seguinte potencial autoconsistente efetivo que minimiza $A\left[n^{\prime}\right]$

$$
v^{\prime}(r)=v(r)+\int \frac{n^{\prime}\left(r^{\prime}\right)}{\left|r-r^{\prime}\right|} \mathrm{d} r^{\prime}+\frac{\delta F_{x c}\left[n^{\prime}\right]}{\delta n^{\prime}(r)}
$$

com $n^{\prime}(r)$ dado por (4.35) e (4.36). A energia livre de Helmholtz fica então, em termos das energias de KS,

$A\left[n^{\prime}\right]=\sum_{i} \varepsilon_{i}^{\prime} f\left(\varepsilon_{i}^{\prime}-\mu\right)-\frac{1}{2} \int v_{h}(r) n^{\prime}(r) \mathrm{d} r-\int v_{x c}(r) n^{\prime}(r) \mathrm{d} r+F_{x c}\left[n^{\prime}\right]-\tau S_{s}\left[n^{\prime}\right]$.

A grandeza de muitos corpos $F_{x c}[n]$, presente nas Eqs. (4.38) e (4.37), análoga ao funcional $E_{x c}[n]$ para $\tau=0$, é muito difícil de se obter. Na aproximação LDA ela é escrita como

$$
F_{x c}[n(r)]=\int n(r) f_{x c}(n(r)) \mathrm{d} r
$$

sendo $f_{x c}(n)$ a contribuição de troca e correlação, por partícula, para a energia livre de um gás de elétrons homogêneo, com densidade $n$, e temperatura $\tau$. No limite de baixas temperaturas, uma primeira aproximação é substituir $f_{x c}$ por seu similar a $\tau=0$, i.e., $\varepsilon_{x c}$. Desta forma, toda a dependência com a temperatura é descrita pelos termos com a função de Fermi $f\left(\varepsilon_{i}\right)$. É justamente esta abordagem 
que eu utilizo neste trabalho.

\subsubsection{Considerações adicionais sobre a DFT}

Nos parágrafos anteriores eu fiz um resumo dos aspectos da teoria do funcional da densidade que são relevantes para este trabalho. Para os três casos analisados, não-magnético, magnético e temperatura finita, eu apenas mostrei como obter o sistema autoconsistente de Kohn-Sham e como escrever a energia total do sistema de muitos corpos em termos das energias monoeletrônicas. A qualidade dos resultados vai depender essencialmente da qualidade das aproximações usadas para a energia de troca e correlação. De um ponto de vista mais aplicado, a formulação de KS mais a LSDA formam uma prescrição prática de como incluir os efeitos de muitos corpos, presentes em sistemas reais, em um cálculo relativamente simples de partículas simples. Antes de encerrar esta seção sobre DFT, algumas consideração adicionais são pertinentes.

Orbitais e energias KS Depois de resolver o sistema KS, é comum supor que as auto-energias e auto-funções resultantes podem ser associadas às correspondentes grandezas do sistema real. Esta hipótese não é garantida pelos teoremas fundamentais da DFT, mas muitas vezes mostra-se válida, em particular nas aplicações para estado sólido. A validade desta associação está, em geral, relacionada com a qualidade do funcional de XC escolhido para descrever o sistema. Como será apresentado na última seção deste capítulo, esta associação entre os orbitais KS e os orbitais do sistema real é um dos ingredientes para a teoria de transporte que utilizo para descrever o magnetotransporte no regime de efeito Hall.

Magnitude e relevância de XC Outro aspecto é sobre a real relevância da contribuição de XC para os heteroestruturas do grupo II-VI que eu investigo. Na Fig. 4.4 eu comparo a magnitude do potencial de troca e correlação, em função da densidade de um gás de elétrons tridimensional, para sistemas dopados baseados em GaAs e ZnSe. Para a faixa de densidade eletrônica típica destes sistemas $\left(n \sim 10^{22} \mathrm{~cm}^{3}\right)$, a magnitude do potencial de XC para sistemas de ZnSe é maior que em sistemas de GaAs. Isto decorre dos valores da massa efetiva $m$ e constante dielétrica $\epsilon$, e consequentemente do Rydberg efetivo $R_{y}^{*}=$ $R_{y}^{0}\left(m / m_{0}\right) /\left(\epsilon / \epsilon_{0}\right)^{2}$ e do raio de Bohr efetivo $a_{0}^{*}=a_{0}\left(\epsilon / \epsilon_{0}\right) /\left(m / m_{0}\right)$ em cada material (valores típicos estão apresentados na Fig. 4.4). Portanto, a relevância da contribuição de XC varia para cada sistema em questão. Como será mostrado nos próximos capítulos, o aspecto mais importante da contribuição de $\mathrm{XC}$ neste 


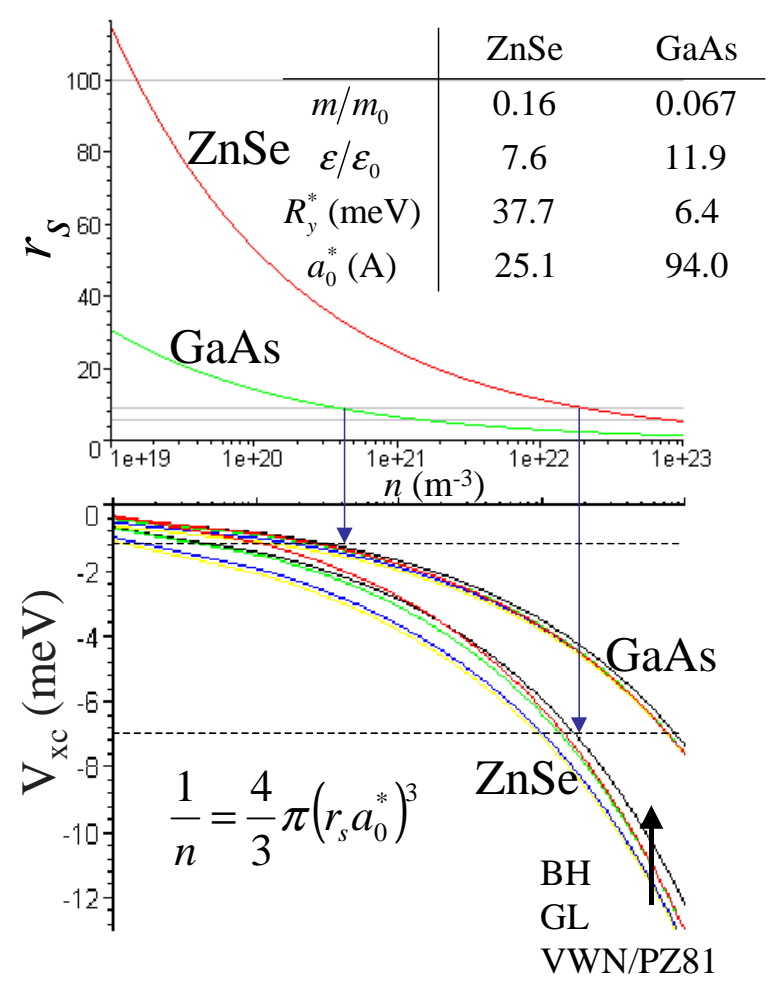

Figura 4.4: Comparação da contribuição de troca e correlação para sistemas de ZnSe e GaAs. O painel superior mostra o parâmetro de densidade $r_{s} \mathrm{em}$ função da densidade para os dois compostos (parâmetros efetivos no inset). À medida que a densidade diminui, $r_{s}$ no ZnSe aumenta muito mais rápido que no GaAs, aproximando-se do limite de validade de várias parametrizações $\left(r_{s} \sim\right.$ 100) mais rapidamente. O painel inferior apresenta o potencial $v_{x c}$ em diversas parametrizações da LSDA: VWN [99], BH [100], GL [101] e PZ81 [102]. No ZnSe (i) o valor do potencial é maior, em módulo, que no GaAs para densidades relevantes $\left(n \sim 10^{22} \mathrm{~m}^{-3}\right.$ ou $\left.r_{s} \sim 5\right)$, e (ii) as diferenças entre as parametrizações são mais acentuadas. As setas ligando os dois gráficos indicam o valor de $v_{x c}$ para $r_{s}=9$.

trabalho foi a sua forte dependência com o spin e com a polarização do gás de elétrons.

Outro aspecto também ilustrado na Fig. 4.4 é que as diferenças entre diversas parametrizações existentes na literatura para o energia/potencial de troca-correlação são mais acentuadas nos sistema de ZnSe que naqueles baseados em GaAs. Da mesma forma que no caso da interpolação de spin, este é outro indicativo da importância de se escolher funcionais apropriados para representar a contribuição de muitos corpos no problema.

Estados estacionários e estados excitados Um último aspecto a ser comentado sobre a DFT é a questão de estados excitados. Este ponto é pertinente pois, no Cap. 6, eu encontro mais de uma solução para as equações de KS, com energias totais diferentes, para um mesmo conjunto de parâmetros externos 
- o que variou foi se o campo magnético estava aumentando ou diminuindo.

Uma vez definida a forma das equações de KS, a resolução destas num ciclo autoconsistente é um problema puramente matemático (ou numérico). Ao resolver numericamente a equação de Schrödinger do sistema KS, não há garantia que a energia encontrada, e a densidade correspondente, corresponda ao estado fundamental. Durante o ciclo autoconsistente, o algoritmo pode encontrar mínimos locais e "achar" que convergiu. De fato o programa convergiu - o que pode ser testado perturbando o sistema, seja mudando o potencial ou a densidade, e verificando se a solução continua a mesma - mas não necessariamente a configuração resultante é a que tem menor energia e, portanto, é o estado fundamental. A questão então é saber se este ponto representa um problema numérico ou se há alguma informação por trás deste resultado.

De fato, Perdew e Levy [115] mostraram que, dado um funcional $E_{v}[n]$ da densidade do estado fundamental associada ao potencial $v$, nem todo estado estacionário $n_{i}$ do sistema de muitos corpos é um extremo de $E_{v}[n]$, mas todo extremo de $E_{v}[n]$ é um estado estacionário $n_{i}$. Se o extremo é um mínimo absoluto ele representa o estado fundamental; senão, o extremo corresponde a um estado excitado. Note que, da mesma forma que o estado fundamental, estes estados excitados são estados reais, físicos, do sistema interagente de muitos corpos. Cooper et al. [34] recentemente interpretou os seus resultados para histerese e relaxação nos seus dados de magnetotransporte em termos de estados metaestáveis.

A interpretação dos resultados do Cap. 6 é baseada nestes resultados da Ref. [115]. O cálculo numérico está retornando estados excitados dependendo das condições iniciais do procedimento autoconsistente. Por um lado, poderia-se sugerir perturbar o ciclo de autoconsistência para não deixar o programa encontrar mínimos locais. Entretanto, o fato destes estados estacionários terem sido encontrados mostra que, pelo menos para uma dada faixa de parâmetros, há espaço de fase disponível para que o sistema acomode-se de várias maneiras possíveis - mínimos locais próximos ao estado fundamental. Ao explorar este espaço de fase, a simulação acaba por reproduzir o ciclo de histerese que foi observada experimentalmente [21].

\subsection{Campo magnético e quantização de Landau}

Nos capítulos 2 e 3 eu considerei o efeito do campo magnético sobre os spins localizados das impurezas de Mn e, indiretamente, sobre os portadores, via a interação de troca $s p-d$. Naqueles capítulos eu desprezei o efeito direto do campo magnético sobre os elétrons e buracos, aproximação comum quando se estuda as 
propriedades magnetoópticas de semicondutores semimagnéticos de gap elevado [51, pág. 288]. Nesta aproximação, e com o confinamento dos poços quânticos apenas na direção de crescimento (longitudinal), o sistema é livre na direção transversal e possui relação de dispersão parabólica. Naqueles capítulos eu estava interessado nas transições ópticas entre as bases das bandas de condução e de valência.

Agora eu estou mudando o enfoque para sistemas altamente dopados que são submetidos a medidas de magnetotransporte na direção transversal (perpendicular à direção de crescimento da amostra). Neste regime, o efeito da força de Lorentz sobre os portadores, induzindo a formação de órbitas de cíclotron, é essencial para descrever os vários fenômenos observados. Em particular, nesta tese eu estudo o caso em que a direção do campo magnético coincide com a direção de crescimento da amostra, i.e., $\boldsymbol{B}=B \hat{z}$. Consequentemente, o movimento orbital induzido por $\boldsymbol{B}$ restringe-se ao plano transversal $x-y$, e o hamiltoniano orbital é completamente separável do hamiltoniano longitudinal (confinamento estrutural e interação de troca $s p-d)$.

\subsubsection{Hamiltoniano orbital}

O tratamento quântico do efeito do campo magnético $\boldsymbol{B}$ sobre os portadores presentes no sistema consiste em reescrever o momento conjugado das equações de movimento incluindo o campo magnético. Para uma partícula com carga $-e$ o momento é $\boldsymbol{P}+e \boldsymbol{A}$, sendo $\boldsymbol{A}$ o potencial vetor correspondente ao campo magnético $\boldsymbol{B}=\nabla \times \boldsymbol{A}$. O hamiltoniano orbital correspondente é então escrito como

$$
H=\frac{1}{2 m}(\boldsymbol{P}+e \boldsymbol{A})^{2} .
$$

Existe uma infinidade de escolhas para o potencial vetor que resultam no mesmo campo magnético. Entretanto, apesar da escolha do gauge determinar a expressão para as funções de onda, os observáveis resultantes sempre serão os mesmos. Usando o gauge de Landau $\boldsymbol{A}=(0, B x, 0)$, a Eq. (4.40) pode ser reescrita como

$$
H \stackrel{\boldsymbol{A}=B x \hat{y}}{\longrightarrow} H_{\perp}=\frac{\hbar^{2} k_{x}^{2}}{2 m}+\frac{1}{2} m \omega_{c}^{2}\left(x-x_{0}\right)^{2},
$$

com dependência espacial apenas no plano $x-y$.

O hamiltoniano transversal (4.41) descreve um oscilador harmônico na direção $\hat{x}$, com frequência de cíclotron $\omega_{c}=e B / m$ e centrado em $x_{0}=-k_{y} \ell_{0}^{2}$, sendo $\ell_{0}=\sqrt{\hbar / e B} \sim 257 B^{-1 / 2} \AA \mathrm{T}^{1 / 2}$ o comprimento magnético. A contribuição do oscilador harmônico para o espectro de energia consiste de níveis equidistantes 
(a) Regular system

$D(E)$

$(H=0)$
$D(E)$

$(H \neq 0)$ (b) Disordered system

$D(E)$

$(H=0)$
$D(E)$

$(H \neq 0)$
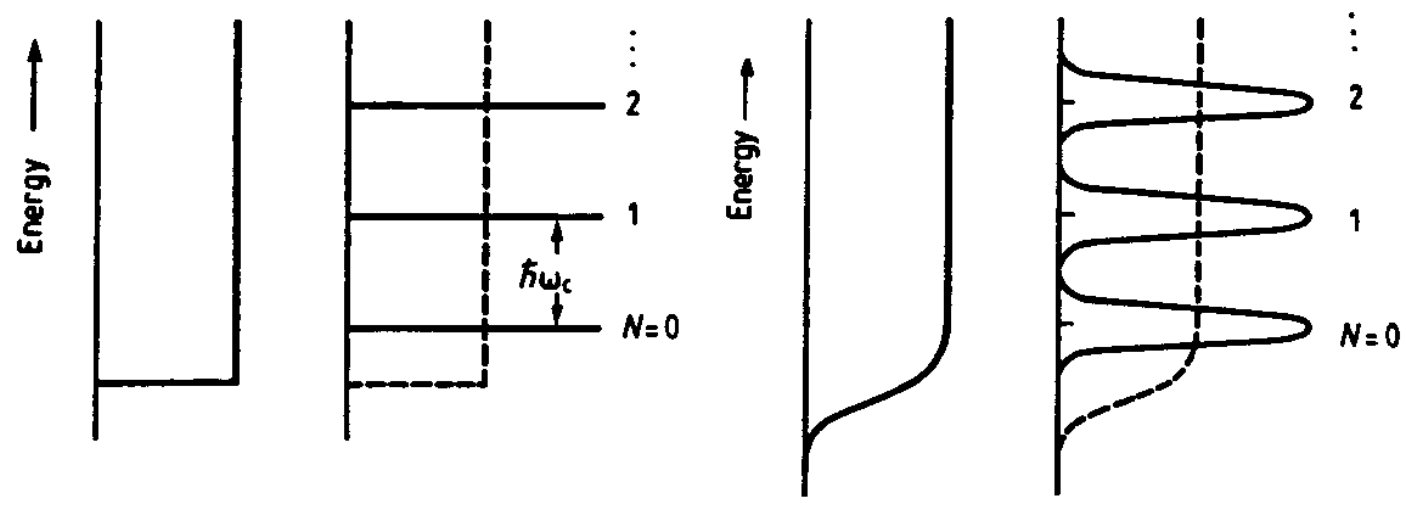

Figura 4.5: Densidade de estados bidimensional sem desordem (a) e com desordem (b). Na ausência de campo magnético a densidade de estados de um gás de elétrons bidimensional é constante. O efeito do campo magnético é colapsar a energia cinética em níveis discretos, níveis de Landau, cujas energias são separadas por $\hbar \omega_{c}$. Quando há desordem no sistema (b), a densidade de estados é alargada. Baseado em Aoki [116].

com energia

$$
\varepsilon_{\perp}=\left(n+\frac{1}{2}\right) \hbar \omega_{c} \quad(n \geq 0),
$$

chamados de níveis de Landau (LL).

As funções de onda correspondentes às soluções estacionárias da equação de Schrödinger para o hamiltoniano (4.41) são dadas por

$$
\varphi_{n, k_{y}}(x, y)=\frac{\mathrm{e}^{\mathrm{i} k_{y} y}}{\sqrt{L_{y}}} \varsigma_{n, k_{y}}\left(x-x_{0}\right) \quad, \quad \varsigma_{n, k_{y}}\left(\bar{x}=x / \ell_{0}\right)=\frac{\mathrm{e}^{-\bar{x}^{2} / 2} H_{n}(\bar{x})}{\sqrt{\ell_{0} 2^{n} \sqrt{\pi} n !}},
$$

nas quais $H_{n}$ são os polinômios de Hermite de ordem $n$.

O vetor de onda $k_{y}$ caracteriza a onda plana presente em $\varphi_{n, k_{y}}(x, y)$. Cada possível estado $k_{y}$ está associado a uma coordenada $x_{0}$ para o centro da órbita do elétron, sem que isto implique em outra energia. Em outras palavras, os estados são degenerados em $k_{y}$. Para contar o número de estados com a mesma energia em uma amostra com área $L_{x} L_{y}$, basta lembrar que as coordenadas das órbitas devem estar dentro da amostra, i.e,

$$
0<x_{0}<L_{x} \quad \rightarrow \quad 0<-k_{y}<\frac{e B L_{x}}{\hbar} \quad \stackrel{\Delta k_{y}=\frac{2 \pi}{L_{y}}}{\longrightarrow} \quad \frac{e B L_{x} / \hbar}{2 \pi / L_{y}}=\frac{e B}{h} L_{x} L_{y}
$$

O número de estados por unidade de área, chamado de degenerescência orbital, 
é então dado por

$$
g_{\square}=\frac{e B}{h} \sim 0.24 \times 10^{11} B \mathrm{~cm}^{-2} \mathrm{~T}^{-1} .
$$

Assim, em um sistema bidimensional na presença de um campo magnético, a densidade de estados possui níveis discretos, contrastando com o caso não magnético em que a densidade de estados bidimensional é constante $g=m / \pi \hbar^{2}{ }^{7}$ A Fig. 4.5(a) ilustra estes dois casos.

\subsubsection{Hamiltoniano Zeeman}

Além do efeito orbital, cuja origem é a interação de Lorentz entre o campo magnético e a carga do portador, há também a interação do campo com o momento magnético de spin do portador. Este é o efeito Zeeman, que induz um desdobramento de spin nos níveis de energia. A contribuição deste efeito para o hamiltoniano é

$$
H_{Z}=\frac{1}{2} g \mu_{B} B \sigma_{z},
$$

em que $g$ é o fator giromagnético e $\mu_{B} \simeq 0.06 \mathrm{meVT}^{-1}$ é o magneton de Bohr. Esta contribuição, da ordem de $\mu_{B}$, é muito pequena quando comparada com o desdobramento de spin devido a interação de troca $s p$-d, que pode ser até duas ordens de magnitude superior. Entretanto, tanto por completeza quanto por ser um termo simples, eu incluo a contribuição Zeeman na formulação do problema.

\subsubsection{Hamiltoniano efetivo total}

Agrupando as várias contribuições para a energia total - termo orbital, Eq. (4.41), o termo Zeeman, Eq. (4.46), e mais um termo longitudinal cujo potencial $v(z)$ tem dependência espacial apenas na direção $\hat{z}$ - o hamiltoniano efetivo total para a função envelope de um portador com massa efetiva $m$, confinado num potencial $v(z)$ e sujeito a um campo magnético externo $\boldsymbol{B}=B \hat{z}$, é escrito como

$$
H=\underbrace{\frac{\hbar^{2} k_{x}^{2}}{2 m}+\frac{1}{2} m \omega_{c}^{2}\left(x-x_{0}\right)^{2}}_{H_{\perp}}+\underbrace{\frac{\hbar^{2} k_{z}^{2}}{2 m}+v(z)}_{H_{\|}}+\underbrace{\frac{1}{2} g \mu_{B} \sigma_{z} B}_{H_{Z}} .
$$

\footnotetext{
${ }^{7}$ Uma rápida dedução da densidade de estados a $B=0$ de um gás de elétrons não interagente consiste em somar sobre todos os estados com $k_{x}$ e $k_{y}$, incluindo a degenerescência de spin:

$$
2 \sum_{k_{x}, k_{y}} 1 \rightarrow 2 \int \frac{\mathrm{d} k_{x}}{2 \pi} \int \frac{\mathrm{d} k_{y}}{2 \pi}=\frac{2}{(2 \pi)^{2}} \int 2 \pi k_{\rho} \mathrm{d} k_{\rho}=\frac{m}{\pi \hbar^{2}} \int \mathrm{d} \varepsilon \Rightarrow g(B=0)=\frac{m}{\pi \hbar^{2}} .
$$

Note que $e B / h=\left(m / \pi \hbar^{2}\right)\left(\hbar \omega_{c}\right)$, mostrando que na presença de $B$, os elétrons que antes ocupavam uma faixa de energia correspondente a $\hbar \omega_{c}$ colapsaram em um único nível altamente degenerado.
} 
As três componentes são completamente separáveis. ${ }^{8}$ As soluções estacionárias são funções envelope

$$
\psi_{i, n, k_{y}}^{\sigma_{z}}(\boldsymbol{r})=\frac{\mathrm{e}^{\mathrm{i} k_{y} y}}{\sqrt{L_{y}}} \varsigma_{n, k_{y}}\left(x-x_{0}\right) \chi_{i}^{\sigma_{z}}(z)\left|\sigma_{z}\right\rangle,
$$

com energias

$$
\varepsilon_{i, n}^{\sigma_{z}}=\varepsilon_{i}^{\sigma_{z}}+\left(n+\frac{1}{2}\right) \hbar \omega_{c}+\frac{1}{2} g \mu_{B} \sigma_{z} B,
$$

sendo $\varepsilon_{i}^{\sigma_{z}}$ a sub-banda resultante do confinamento longitudinal $\left(H_{\|}\right),(n+1 / 2) \hbar \omega_{c}$ a energia dos orbitais de Landau $\left(H_{\perp}\right)$, e $g \mu_{B} \sigma_{z} B$ a contribuição Zeeman $\left(H_{Z}\right)$.

Com os termos orbital e Zeeman resolvidos e separados do termo longitudinal, na prática é preciso resolver apenas a equação de Schrödinger unidimensional correspondente ao termo $H_{\|}$para obter as energias de sub-banda $\varepsilon_{i}^{\sigma_{z}}$ e as funções envelope $\chi_{i}^{\sigma_{z}}(z)$.

\subsubsection{Densidade de estados}

Os estados com energia $\varepsilon_{i, n}^{\sigma_{z}}$ são altamente degenerados [cf. Eq. (4.45)]. No caso ideal de uma amostra completamente uniforme e sem impurezas, a densidade de estados (DOS - density of states) é uma sucessão de níveis discretos [Fig. 4.5(a)],

$$
g(\varepsilon)=\frac{e B}{h} \sum_{i, n, \sigma_{z}} \delta\left(\varepsilon-\varepsilon_{i, n}^{\sigma_{z}}\right)
$$

Na prática, existem impurezas espalhadoras, defeitos na rede e variações nas interfaces que quebram a uniformidade do sistema. Isto acaba por gerar um potencial de desordem aleatório $V_{d}(\mathbf{r})$ que deve ser somado ao hamiltoniano ideal $[116,117]$, ou seja,

$$
H \rightarrow H+V_{d}(\boldsymbol{r})
$$

Designando a coordenada do centro do movimento de cíclotron por $(X, Y)$, as equações de movimento serão [116]

$$
\dot{X}=\frac{\mathrm{i}}{\hbar}\left[V_{d}, X\right]=\frac{\ell_{0}^{2}}{\hbar} \frac{\partial V_{d}}{\partial y}, \quad, \quad \dot{Y}=\frac{\mathrm{i}}{\hbar}\left[V_{d}, Y\right]=-\frac{\ell_{0}^{2}}{\hbar} \frac{\partial V_{d}}{\partial x} .
$$

\footnotetext{
${ }^{8}$ Para os DMWQs estudados nesta tese, o potencial $v(z)$ consiste nos termos de confinamento estrutural e da interação de troca sp-d, Eq. (3.2), mais os termos da interação de Coulomb apresentados na seção 4.2. Como visto nos capítulos anteriores, os termos de confinamento estrutural e de troca sp-d têm dependência espacial apenas na direção $\hat{z}$. Mais adiante neste capítulo eu indico quais são as condições para que eu também considere a dependência espacial da interação de Coulomb apenas na direção longitudinal, o que permite a separação das equações de movimento nas direções transversal e longitudinal.
} 
O movimento orbital passa a ser sobre as equipotenciais do potencial $V_{d}$ e a degenerescência dos estados com diferentes $(X, Y)$ de um dado nível de Landau é levantada. Consequentemente, a DOS deixa de ser discreta e forma uma banda com largura característica $\Gamma$ (sub-banda de Landau), ilustrada na Fig. 4.5(b).

As equações de movimento (4.52) deixam claro que a DOS das subbandas de Landau dependem fortemente da variação espacial do potencial aleatório $V_{d}(\boldsymbol{r})$. Por exemplo, para o caso de espalhadores de curto alcance $\left[V_{d}(\boldsymbol{r})\right.$ varia numa escala menor que $\ell_{0}$ ], Ando e Uemura [118] mostraram $^{9}$ que o perfil da DOS é de semi-elipses com largura característica

$$
\Gamma=\sqrt{\frac{2}{\pi} \hbar \omega_{c} \frac{\hbar}{\tau}}=\frac{\hbar e}{m} \sqrt{\frac{2}{\pi} \frac{B}{\mu}} \sim \frac{0.09}{m / m_{0}} \sqrt{\frac{B}{\mu}} \mathrm{meV},
$$

na qual $\tau=\mu m / e$ é o tempo de espalhamento e $\mu$ é a mobilidade. Em (4.53) $B$ está em unidades de $\mathrm{T}$ e a mobilidade $\mu$ em unidades de $\mathrm{m}^{2} / \mathrm{Vs}$. Outros modelos utilizam densidades de estados gaussianas [119-124] e lorentzianas [125]. Nesta tese eu adoto um perfil gaussiano para a DOS, seguindo a Ref. [122],

$$
g(\varepsilon)=\sum_{i, n, \sigma_{z}} g_{i, n}^{\sigma_{z}}(\varepsilon)=\sum_{i, n, \sigma_{z}} \frac{e B}{h} \frac{1}{\Gamma \sqrt{2 \pi}} \exp \left[-\frac{\left(\varepsilon-\varepsilon_{i, n}^{\sigma_{z}}\right)^{2}}{2 \Gamma^{\sigma_{z}^{2}}}\right]
$$

cuja largura característica $\Gamma^{\sigma_{z}}$ de cada nível ${ }^{10}$ é diferenciada para cada sub-banda de spin. A Fig. 4.5(b) ilustra este perfil gaussiano para a DOS. Aqui eu não estou interessado em investigar os detalhes dos mecanismos de espalhamento em DMHs e a consequência disto para o perfil da DOS. Assim, eu preciso de uma descrição fenomenológica que permita incluir o alargamento dos LLs no meu modelo. A escolha pelo perfil gaussiano foi motivada, por um lado, pelos trabalhos teóricos que resultam neste tipo de perfil quando consideram o efeito do espalhamento por impurezas na DOS e no transporte [119-121, 123, 124], e por outro lado, por questões práticas de implementação, uma vez que a gaussiana é uma função "bem comportada".

Quanto a dependência do alargamento $\Gamma^{\sigma_{z}}$ com o spin, uma vez que há interações magnéticas dependentes de spin (troca $s p-d$ ) nas heteroestruturas magnéticas aqui investigadas, é razoável considerar que existam mecanismos de espalhamento que também dependam do spin. Por exemplo, no Cap. 3 eu mostro que os tempos de espalhamento de spin são diferentes para spins majoritários e

\footnotetext{
${ }^{9}$ Ando e Uemura [118] efetuaram um cálculo perturbativo usando o formalismo de funções de Green na aproximação autoconsistente de Born.

${ }^{10}$ Note que $\Gamma^{\sigma_{z}}$ é de fato a meia-largura da função gaussiana na Eq. (4.54), enquanto $\Gamma$ calculado por Ando e Uemura [118], Eq. (4.53), é a largura total do LL. Assim, seu eu quiser utilizar (4.53) em (4.54) preciso fazer $\Gamma^{\sigma_{z}}=\Gamma / 2$.
} 
minoritários.

Densidade de portadores Uma vez obtida a estrutura eletrônica e a densidade de estados, posso calcular a densidade de portadores. Ela é obtida simplesmente calculando o módulo da função de onda ao quadrado, i.e.,

$$
n(\boldsymbol{r})=\sum_{i, n, k_{y}, \sigma_{z}}\left|\psi_{i, n, k_{y}}^{\sigma_{z}}(\boldsymbol{r})\right|^{2}=\sum_{i, n, k_{y}, \sigma_{z}}\left|\chi_{i}^{\sigma_{z}}(z)\right|^{2}\left|\varphi_{n, k_{y}}(x, y)\right|^{2}
$$

Esta densidade é função não apenas da coordenada longitudinal (z), mas também das coordenas transversais $(x, y)$. Assim, se eu utilizar esta densidade na formulação da DFT/LSDA apresentada na Sec. 4.2, os potenciais $v_{h}([n] ; r)$ e $v_{x c}\left(\left[n_{\uparrow}, n_{\downarrow}\right] ; r\right)$ serão funções das três coordenas espaciais. Feito assim, eu não poderia fazer a separação de variáveis que efetuei na Eq. (4.47). Assim, aqui eu faço uma aproximação adicional que é fazer uma média sobre a densidade eletrônica no plano $x-y$. Este procedimento de média é fisicamente justificado uma vez que (i) as órbitas de Landau podem estar centradas em qualquer ponto no plano $x-y$, e (ii) no magnetotransporte transversal, que é a propriedade que estou interessado, as correntes medidas são grandezas "integradas", i.e., contêm a contribuição de todos os portadores que fazem parte da condução.

A média da parte transversal da densidade (4.55) sobre o plano $x-y$, efetuada na área $L_{x} L_{y}$, é simplesmente

$$
\frac{1}{L_{x} L_{y}} \int_{L_{x}} \int_{L_{y}}\left|\varphi_{n, k_{y}}(x, y)\right|^{2} \mathrm{~d} x \mathrm{~d} y=\frac{1}{L_{x} L_{y}} .
$$

Desta forma a densidade média fica

$$
n(z)=\frac{1}{L_{x} L_{y}} \sum_{i, n, \sigma_{z}} \sum_{k_{y}}\left|\chi_{i}^{\sigma_{z}}(z)\right|^{2}
$$

A soma em $k_{y}$, que eu deixei em destaque, "conta" o número de estados em cada nível de Landau. Agora é mais conveniente substituir esta soma pela integral na densidade de estados (4.54). Para um sistema de área $L_{x} L_{y}$, a densidade de estados por unidade de energia em cada LL é $L_{x} L_{y} g_{i, n}^{\sigma_{z}}(\varepsilon)$, de forma que a densidade fica

$$
n(z)=\frac{1}{L_{x} L_{y}} \sum_{i, n, \sigma_{z}}\left|\chi_{i}^{\sigma_{z}}(z)\right|^{2} \int_{-\infty}^{\varepsilon_{F}} L_{x} L_{y} g_{i, n}^{\sigma_{z}}(\varepsilon) \mathrm{d} \varepsilon .
$$

Esta expressão é para temperatura zero e, portanto, a integral é efetuada até o nível de Fermi $\varepsilon_{F}$. A extensão para temperaturas finitas é feita utilizando a 
função de Fermi

$$
f(\varepsilon)=\frac{1}{1+\exp \left[(\varepsilon-\mu) / k_{B} T\right]},
$$

sendo $\mu$ o potencial químico. ${ }^{11}$ Assim, a expressão para a densidade fica

$$
n(z)=\sum_{i, n, \sigma_{z}}\left|\chi_{i}^{\sigma_{z}}(z)\right|^{2} \int_{-\infty}^{\infty} g_{i, n}^{\sigma_{z}}(\varepsilon) f(\varepsilon) \mathrm{d} \varepsilon .
$$

Esta grandeza é função apenas da coordenada $z$, formalmente permitindo a separação do problema em uma parte transversal e outra longitudinal. Isto simplifica o cálculo, uma vez permite que eu resolva as equações de Kohn-Sham apenas na direção de crescimento, ou seja, em uma dimensão.

Densidade bidimensional Integrando a densidade $n(z)$, Eq. (4.60), na direção $\hat{z}$, eu obtenho as densidades bidimensionais parciais $n_{2 D}^{\sigma_{z}}$ de cada componente de spin e a densidade bidimensional total $n_{2 D}$, ou seja,

$$
n_{2 D}=\sum_{\sigma_{z}}\left[\int_{-\infty}^{\infty} \sum_{i, n} g_{i, n}^{\sigma_{z}}(\varepsilon) f(\varepsilon) \mathrm{d} \varepsilon\right]=n_{2 D}^{\uparrow}+n_{2 D}^{\downarrow} .
$$

Com estas densidades parciais $n_{2 D}^{\sigma_{z}}$ define-se a polarização

$$
\zeta=\frac{n_{2 D}^{\uparrow}-n_{2 D}^{\downarrow}}{n_{2 D}}
$$

A densidade $n_{2 D}$ é usualmente medida usando o efeito Hall clássico (baixo campo). Neste regime, a resistividade Hall em função do campo magnético é uma reta com coeficiente angular inversamente proporcional a $n_{2 D}$. Valores típicos são $n_{2 D} \sim 10^{11} \mathrm{~cm}^{-2}$.

\subsubsection{Energia orbital e magnetização}

Para finalizar esta seção sobre a quantização orbital em um 2DEG, eu mostro qual é a dependência da energia total orbital e da magnetização orbital em função do campo magnético. Estas duas grandezas serão necessárias para discutir as medidas de magnetização dos experimentos analisados no Cap. 5.

No caso mais simples de um 2DEG não interagente, o hamiltoniano é dado por (4.41) e o espectro de energia é $\varepsilon_{n}(B)=(n+1 / 2) \hbar \omega_{c}$. A energia

\footnotetext{
${ }^{11}$ Aqui eu utilizo o símbolo $\mu$ para designar o potencial químico. Em outros contextos este mesmo símbolo é usado para a mobilidade.
} 

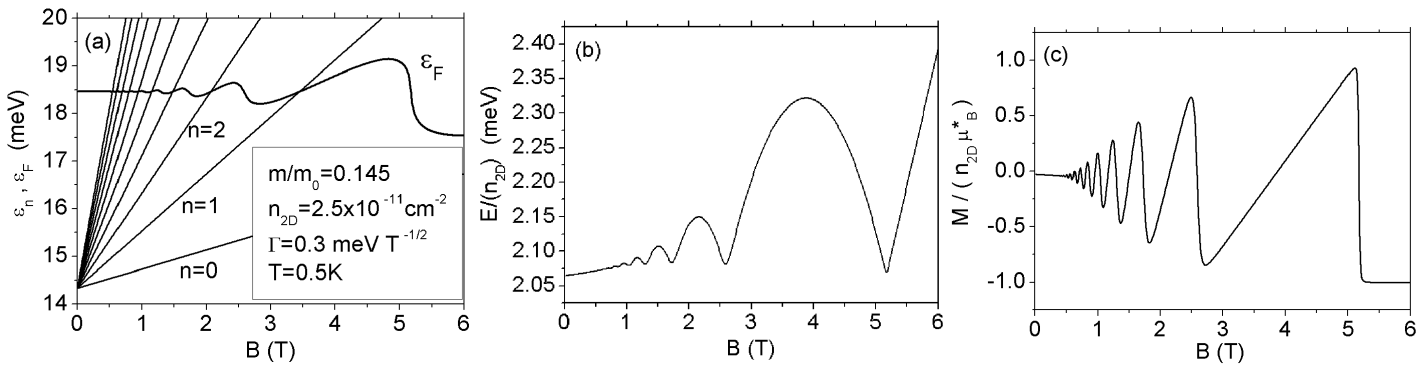

Figura 4.6: Diagrama de níveis de Landau (a), energia orbital (b) e magnetização orbital (c) de uma gás de elétrons bidimensional não-interagente obtidos com o programa numérico desenvolvido para esta tese. Neste exemplo, o gás de elétrons está confinado em um poço quântico de altura $v_{0}$. Assim, a energia em $B=0$ no diagrama de níveis de Landau é a energia da sub-banda. A densidade total é constante e, portanto, em (a) o potencial químico oscila em função de $B$. Este comportamento de $\mu$ se reflete na energia orbital total (b) e na magnetização (c). As oscilações da magnetização são conhecidas como o efeito de Haas-van Alphen. Os parâmetros usados neste exemplo são $n_{2 D}=2.5 \times 10^{11} \mathrm{~cm}^{-2}, \mathrm{~m} / \mathrm{m}_{0}=0.145$, $v_{0}=120 \mathrm{meV}, \Gamma=0.3 B^{1 / 2} \mathrm{meV} \mathrm{T}^{-1 / 2}$ e $T=0.5 \mathrm{~K}$.

orbital total é dada por

$$
E=\int_{-\infty}^{+\infty} \varepsilon g(\varepsilon) f(\varepsilon) \mathrm{d} \varepsilon
$$

na qual $g(\varepsilon)$ é a densidade de estados [Eq. (4.50) no caso discreto ou Eq. (4.54) para o caso com alargamento].

A Fig. 4.6(a) ilustra o diagrama de níveis de Landau para este caso não-interagente. Neste exemplo, a densidade é constante e, portanto, o nível de Fermi oscila em função de $B$ enquanto percorre os LLs - lembre que cada LL comporta $e B / h$ elétrons e, portanto, para $B$ 's maiores é necessário menos LLs para uma dada densidade total $n_{2 D}$. O painel (b) mostra a energia orbital total dada pela Eq. (4.63). As oscilações ocorrem porque, à medida que o campo magnético aumenta, o nível de Fermi "salta" para energias mais baixas.

Usando a energia total eu posso obter a magnetização orbital do sistema. A magnetização reflete como a energia varia com o campo magnético. Ela é dada por

$$
M=-\left.\frac{\partial E}{\partial B}\right|_{n_{2 D}} .
$$

É importante notar que a magnetização é a derivada parcial da energia, mantendo as demais variáveis constantes. No caso que estou apresentando aqui, nãointeragente e não-magnético, a energia é função apenas do campo magnético e da densidade. Neste caso, a derivada parcial é igual à própria derivada total e, para obter a curva da magnetização, basta efetuar a derivada da curva da Fig. 4.6(b) em relação a $B$. Num caso mais geral, por exemplo quando há desdobramento 
nos níveis de spin e as densidades parciais de cada componente de spin variam $\operatorname{com} B$, a derivada parcial deve ser tomada apropriadamente [126].

A Fig. 4.6(c) ilustra a magnetização de um 2DEG não-interagente e não-magnético. As oscilações da magnetização são, novamente, devido a passagem do nível de Fermi por sucessivos LLs à medida que o campo magnético varia. Este efeito é conhecido como de Haas-van Alphen (dHvA) [114, 126-129].

\subsection{Magnetotransporte no regime quântico}

Efeitos resultantes da quantização da energia cinética pelo campo magnético quantização de Landau - são conhecidos a muito tempo. Já em 1930 de Haas e van Alphen mediram oscilações na magnetização em metais que tinham periodicidade de 1/B - oscilações de de Haas-van Alphen. A condutividade também apresentava oscilações - oscilações de Shubnikov-de Haas-, bem como quaisquer outras grandezas que fossem medidas com precisão suficiente [114, Cap. 14].

Com o aperfeiçoamento das técnicas de crescimento de heteroestruturas semicondutoras, estes mesmos tipos de experimentos foram realizados em 2DEGs. A quantização espacial somada à quantização orbital trouxe a tona os efeitos Hall quânticos, inteiro [90] e fracionário [91, 92]. Eles eram surpreendentes pois iam além dos modelos existentes até então para explicar transporte no regime quântico [118, 123, 124, 130-132].

Com exceção da primeira parte desta tese, Caps. 2 e 3, os demais capítulos envolvem resultados de magnetotransporte no regime do efeito Hall quântico inteiro (IQHE - integer quantum Hall effect). Experimentos de magnetotransporte consistem em estudar o transporte elétrico na presença de um campo magnético. Basicamente, aplica-se uma diferença de potencial entre terminais e mede-se a corrente resultante. O comportamento da resistividade fornece informações sobre o sistema como, por exemplo, a mobilidade e densidade de portadores. Existem dois regimes para este efeito: o clássico, que ocorre a baixos campos, e o quântico, que ocorre quando há quantização de Landau. Nos parágrafos seguintes eu descrevo estes dois regimes resumidamente e, em seguida, apresento o modelo que utilizo para simular o magnetotransporte no regime quântico.

\subsubsection{Efeito Hall clássico}

Em 1879 Hall [133] descobriu que, ao se aplicar um campo elétrico e magnético sobre um condutor, como ilustrado na Fig. 4.7, estabelece-se um campo elétrico perpendicular ao aplicado. A força de Lorentz $-e \boldsymbol{V} \times \boldsymbol{B}$ exerce uma força na direção transversal sobre os elétrons em movimento, de forma que eles se acumu- 


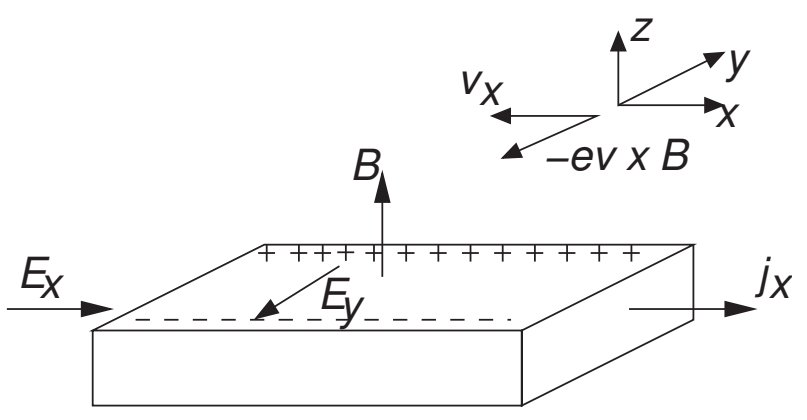

Figura 4.7: Diagrama da geometria Hall. O campo elétrico $E_{x}$ induz uma corrente ao longo da amostra. Devido ao campo magnético na direção $\hat{z}$ os elétrons são defletidos e se acumulam numa das extremidades da amostra. O campo elétrico $E_{y}$ acaba por anular a força de Lorentz $e \boldsymbol{v} \times \boldsymbol{B}$ de forma que a corrente resultante continua na direção $\hat{x}$. A diferença de potencial na direção $\hat{y}$ é chamada de voltagem Hall.

lam em um dos lados do condutor, dando origem ao campo elétrico transversal. Quando este campo é suficiente para anular a força de Lorentz, a corrente flui apenas na direção do campo elétrico aplicado externamente.

No âmbito do modelo de Drude [veja o Quadro (1)], a resistividade longitudinal $\rho_{x x}=E_{x} / j_{x}$ é independente do campo magnético aplicado. Já a resistividade transversal $\rho_{x y}=E_{y} / j_{x}$ é proporcional ao campo magnético, sendo a constante de proporcionalidade (coeficiente Hall $R_{H}=-1 / n_{2 D} e$ ) inversamente proporcional à densidade $n_{2 D}$ de elétrons e à carga fundamental $e(>0)$. Esta última característica é particularmente interessante por toda história da física relacionada com o efeito Hall. Primeiro, porque o sinal do coeficiente Hall indica o sinal da carga dos portadores (resultado que confirmou o sinal da carga do elétron determinado por Thomson anos mais tarde). Além disso, a medida deste coeficiente em alguns metais apresentava um sinal oposto, indicando que a carga dos portadores tinham um sinal oposto ao do elétron; esta questão só veio ser esclarecida com a teoria quântica dos sólidos (conceitos bandas de energia e de buracos). O segundo ponto interessante é a possibilidade de se medir a densidade eletrônica; para amostras suficientemente puras, e em baixas temperaturas, o resultado é extremamente preciso, sendo este, atualmente, um método padrão para determinar a densidade eletrônica em sistemas nanoscópicos bidimensionais em baixos campos magnéticos [Eq. (4.70b)].

\subsubsection{Efeitos Hall quântico inteiro e Shubnikov-de Haas}

Efeito Hall quântico inteiro Quando o efeito Hall é medido em campos magnéticos altos, a baixas temperaturas $(T \lesssim 4.2 \mathrm{~K})$, e em amostras finas o suficiente tal que o movimento dos elétrons é confinado a um plano, e.g. 
Quadro 1: Modelo de Drude para o efeito Hall clássico [134, pág. 93] No equilíbrio, os elétrons recebem momento do campo na mesma taxa que perdem devido aos espalhadores. Assim, na presença dos campos $\boldsymbol{E}$ e $\boldsymbol{B}$, e designando o tempo de relaxação de momento por $\tau_{m}$, tenho

$$
\frac{m \mathbf{v}_{d}}{\tau_{m}}=e\left[\boldsymbol{E}+\mathbf{v}_{d} \times \boldsymbol{B}\right] .
$$

Reescrevendo na forma matricial

$$
\left[\begin{array}{cc}
m / e \tau_{m} & -B \\
+B & m / e \tau_{m}
\end{array}\right]\left(\begin{array}{l}
v_{x} \\
v_{y}
\end{array}\right)=\left(\begin{array}{c}
E_{x} \\
E_{y}
\end{array}\right)
$$

A densidade de corrente $\boldsymbol{J}$ (por unidade de comprimento) é relacionada com a densidade $n_{2 D}$ por $\boldsymbol{J}=e \mathbf{v}_{d} n_{2 D}$. Assim,

$$
\left[\begin{array}{cc}
m / e \tau_{m} & -B \\
+B & m / e \tau_{m}
\end{array}\right]\left(\begin{array}{l}
J_{x} / e n_{2 D} \\
J_{y} / e n_{2 D}
\end{array}\right)=\left(\begin{array}{c}
E_{x} \\
E_{y}
\end{array}\right)
$$

que pode ser reescrito como

$$
\left(\begin{array}{l}
E_{x} \\
E_{y}
\end{array}\right)=\sigma^{-1}\left[\begin{array}{cc}
1 & -\mu B \\
+\mu B & 1
\end{array}\right]\left(\begin{array}{l}
J_{x} \\
J_{y}
\end{array}\right)
$$

$\operatorname{com} \sigma \equiv|e| n_{2 D} \mu$ e $\mu \equiv|e| \tau_{m} / m$. Usando a definição do tensor de resistividade

$$
\begin{gathered}
\left(\begin{array}{l}
E_{x} \\
E_{y}
\end{array}\right)=\left[\begin{array}{ll}
\rho_{x x} & \rho_{x y} \\
\rho_{y x} & \rho_{y y}
\end{array}\right]\left(\begin{array}{l}
J_{x} \\
J_{y}
\end{array}\right), \\
\rho_{x x}=\sigma^{-1}, \\
\rho_{y x}=-\rho_{x y}=\mu B / \sigma=B / e n_{2 D} .
\end{gathered}
$$

tenho

O modelo de Drude prediz que a resistividade longitudinal é constante enquanto a resistividade Hall é linear com $B$. As medidas de efeito Hall são procedimentos padrões na caracterização de sistemas semicondutores pois fornecem a densidade $n_{2 D}$ e a mobilidade $\mu$ separadamente, enquanto medidas a campo nulo fornecem apenas o produto $n_{2 D} \mu$.

$x-y$, a resistência Hall desvia dramaticamente do valor clássico. Em campos suficientemente altos surgem platôs bem definidos nos gráficos de $\rho_{x y}$ em função do campo magnético [veja, e.g., as Figs. 5.1 e 6.2(a) nos capítulos seguintes, págs. 113 e 139, respectivamente]. O valor da resistividade nestes platôs é exatamente quantizado em sub-harmônicos de $h / e^{2}$, sendo $h$ a constante de Planck e $e$ a carga do elétron. Este é o efeito Hall quântico inteiro e foi descoberto por von Klitzing, Dorda, e Pepper [90] em 1980. O IQHE é um efeito universal pois é independente do material usado e do formato da amostra, sendo função apenas de constantes físicas universais. ${ }^{12}$

Efeito Shubnikov-de Haas A resistividade longitudinal também apresenta um comportamento peculiar quando medida em condições semelhan-

\footnotetext{
${ }^{12}$ Por ser um fenômeno tão robusto, com precisão de pelo menos uma parte por milhão, o padrão internacional de resistência é definido em termos do IQHE.
} 

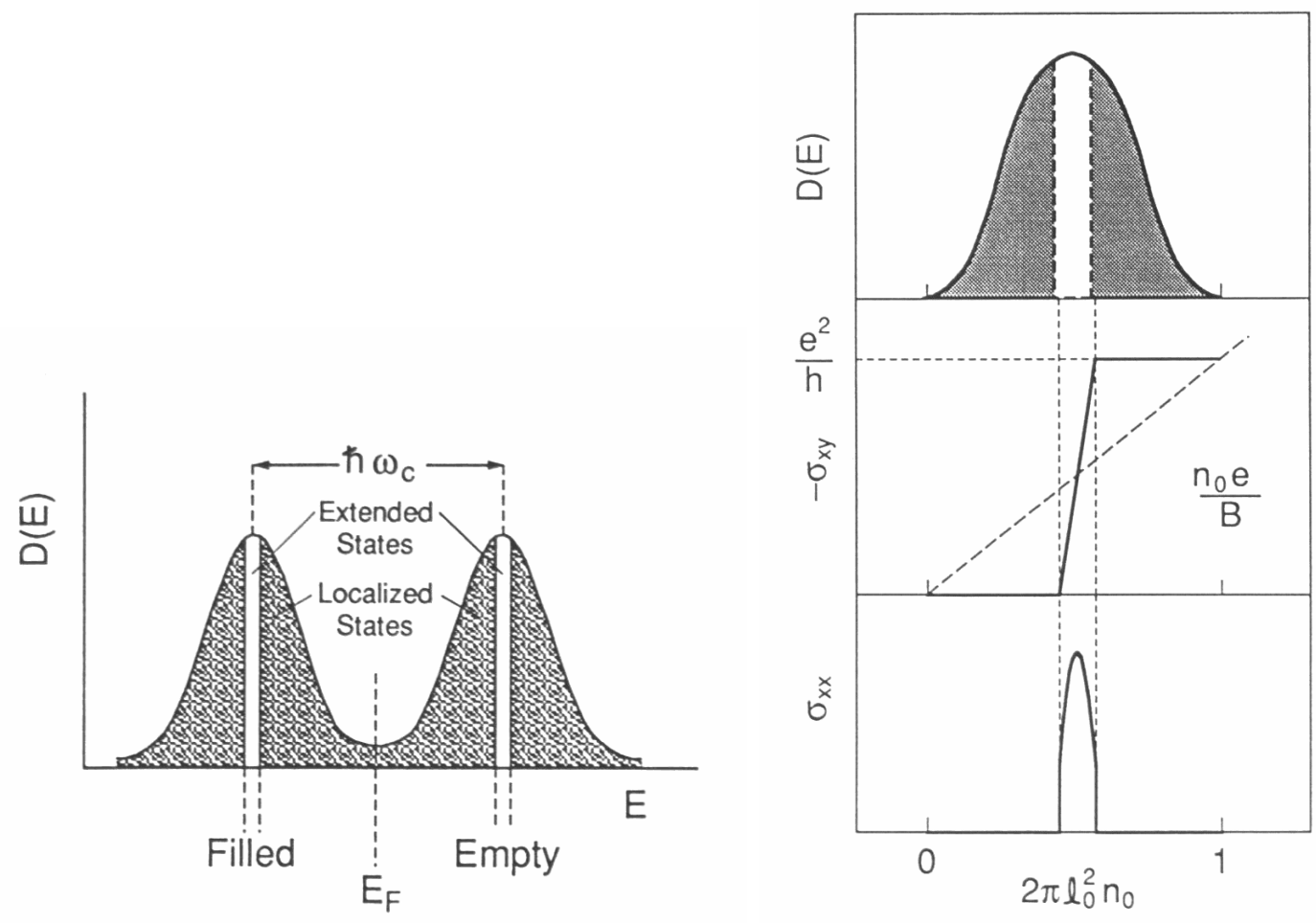

Figura 4.8: Explicação para os platôs o efeito Hall em termos de estados localizados. (Esquerda) Densidade de estados de dois níveis de Landau separados por $\hbar \omega_{c}$ contendo níveis estendidos e localizados. Os estados abaixo do nível de Fermi estão preenchidos. (Direita) Densidade de estados $D(E)$, condutividade Hall $\sigma_{x y}$ e condutividade longitudinal $\sigma_{x x}$ em função do fator de preenchimento. Enquanto o nível de Fermi percorre estados localizados $\sigma_{x y}$ é constante (platô) e $\sigma_{x x}=0$. Quando $E_{F}$ percorre estados estendidos ocorre condução. Consequentemente há uma resistividade $\sigma_{x x} \neq 0$ e ocorre uma transição para um novo platô em $\rho_{x y}=n_{0} e / B$, em que $n_{0}$ é a densidade de um nível de Landau completamente ocupado. Extraído de Chakraborty e Pietiläinen [135].

tes à do IQHE. Nas mesmas faixas de campo magnético em que a resistividade Hall é constante, a resistividade longitudinal se anula [e.g. Figs. 5.2 e 6.2(a), págs. 114 e 139]; nas regiões intermediárias ocorrem picos de resistividade. Estas oscilações da resistividade longitudinal são conhecidas como efeito Shubnikov-de Haas (SdH). Este efeito, que foi observado numa grande variedade de amostras macroscópicas e heteroestruturas, também tem origem quântica e é uma evidência de que o campo magnético induz singularidades na densidade de estados do sistema.

Papel das impurezas $\mathrm{O}$ efeito Hall quântico inteiro se manifesta no regime de quantização orbital (LL) e espacial (2DEG), mas só estes elementos não explicam o aparecimento dos platôs na resistividade Hall. Entre $1972 \mathrm{e}$ 1975 Tsuneya Ando e colaboradores [118, 123, 130-132] publicaram uma série 
de trabalhos estabelecendo a base teórica para transporte quântico em sistemas bidimensionais. Efeitos oscilatórios em $B$ surgem naturalmente da teoria, mas não reproduzem os resultados para as resistividades no regime de IQHE.

O elemento que faltava era o papel das impurezas nestes sistemas [117]. Além de alargar os níveis eletrônicos devido a espalhamentos, estas mesmas impurezas localizam a maioria dos estados eletrônicos. Enquanto os estados localizados não carregam corrente, os estados com energia próxima ao centro do nível de Landau permanecem estendidos e, portanto, conduzem corrente. À medida que o potencial químico do sistema é variado, seja mudando o campo magnético, ou a densidade do 2DEG, ele percorre regiões de estados localizados, onde não há dissipação e a resistividade é nula, e regiões de estados estendidos, em que há corrente e, portanto, a resistividade é finita (Fig. 4.8). No IQHE, os platôs de condutividade ocorrem quando o potencial químico está sobre estados localizados. Entretanto, o valor da resistividade nestas regiões é independente dos detalhes da estrutura de banda e da quantidade de estados que estão estendidos e localizados: ele é definido apenas pelo número de LLs com estados estendidos abaixo do nível de Fermi. Definindo o fator de preenchimento como

$$
\nu=\frac{n_{2 D}}{e B / h}
$$

e usando a Eq. (4.70b), tenho que

$$
\rho_{x y}=\frac{B}{n_{2 D} e}=\frac{h}{\nu e^{2}} .
$$

Este é justamente o resultado esperado se todos os estados (estendidos + localizados) contribuíssem para para corrente Hall. Prange [136] explicou este paradoxo mostrando que a formação de um estado localizado é compensada por um aumento apropriado na corrente Hall dos estados estendidos remanescentes.

\subsubsection{Modelo para o magnetotransporte}

Para modelar o magnetotransporte eu utilizo o modelo proposto por Ando e Uemura [118]. O modelo aplica a teoria de transporte de Kubo (resposta linear) para transporte em um sistema quântico bidimensional, e considera espalhadores de curto alcance na aproximação autoconsistente de Born. Este modelo, como proposto originalmente, não reproduz o IQHE. Para usar este modelo eu incluo o efeito das impurezas considerando separadamente duas densidades de estados [122]:

I. A DOS total $g(\varepsilon)$ da Eq. (4.54), com alargamento $\Gamma^{\sigma_{z}}$, que compreende 


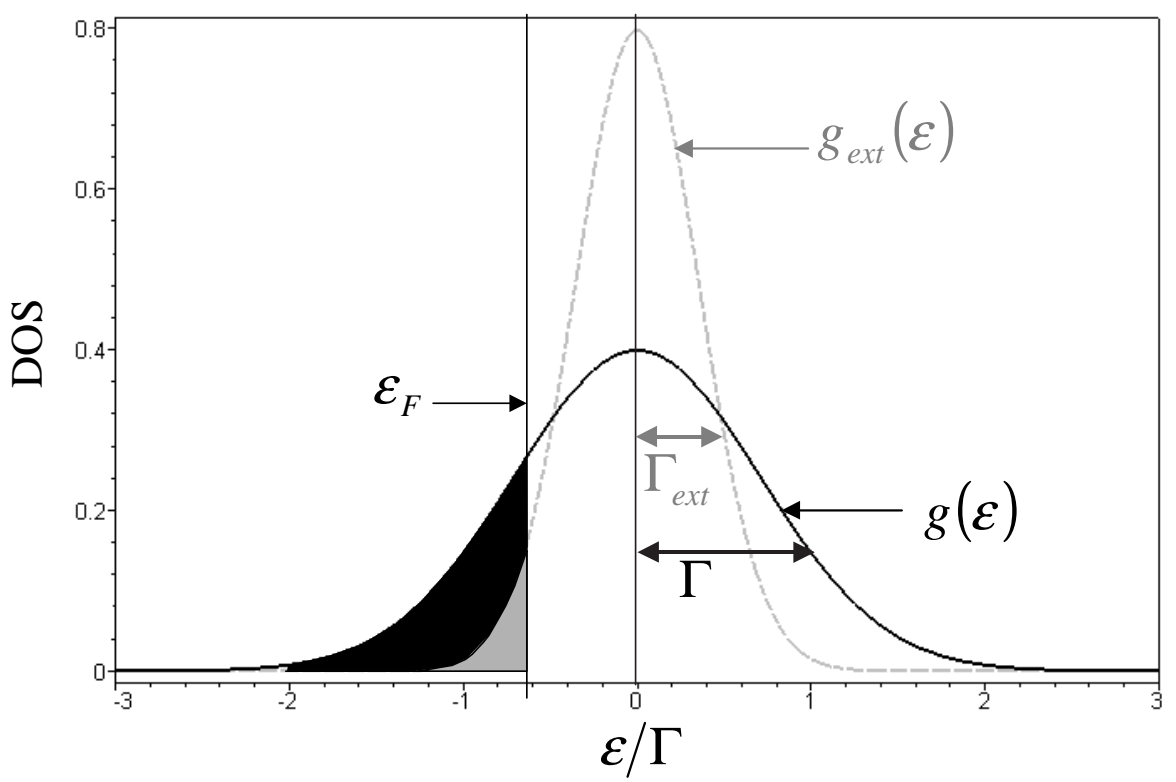

Figura 4.9: Densidades de estados total $g(\varepsilon)$ com largura característica $\Gamma$ e densidade de estados estendidos $g_{\text {ext }}(\varepsilon)$ com largura $\Gamma_{\text {ext }}$. Os estados com energia até o nível de Fermi $\varepsilon_{F}$ estão ocupados (área preta mais a área cinza). Entretanto, apenas os estados estendidos (área cinza) contribuem para o transporte.

todos os estados, estendidos e localizados, é utilizada para determinar a posição do nível de Fermi (ou potencial químico $\mu$ quando $T \neq 0$ ). ${ }^{13}$

II. A outra DOS é para os estados estendidos,

$$
g_{e x t}(\varepsilon)=\frac{e B}{h} \sum_{i, n, \sigma_{z}} \frac{1}{\Gamma \sqrt{2 \pi}} \exp \left[-\frac{\left(\varepsilon-\varepsilon_{i, n}^{\sigma_{z}}\right)^{2}}{2 \Gamma_{e x t}^{\sigma_{z}}{ }^{2}}\right],
$$

com alargamento $\Gamma_{e x t}^{\sigma_{z}}<\Gamma^{\sigma_{z}}$, simulando os estados estendidos com energias no centro dos LLs. Note que, apesar das larguras diferentes, $g_{e x t}(\varepsilon)$ contribui com a mesma quantidade de estados que a DOS total $g(\varepsilon)$.

A Fig. 4.9 ilustra as duas DOS para um nível de Landau. Abaixo da energia de Fermi todos os níveis estão ocupados, mas só a fração correspondente a $g_{\text {ext }}$ contribui para o transporte. Note que, como $g_{\text {ext }}(\varepsilon)$ tem o mesmo número de estados que $g(\varepsilon)$, mas concentrados numa faixa mais estreita de energia, utilizar $g_{\text {ext }}(\varepsilon)$ é uma forma fenomenológica de simular a teoria de Prange [136] na qual os estados estendidos no centro dos LLs contribuem como se a totalidade dos

\footnotetext{
${ }^{13}$ Nos sistemas dopados por modulação duas situações podem ocorrer. Quando as camadas dopadas são parcialmente depletadas, o potencial químico $\mu$ permanece fixo, determinado pelo nível das impurezas doadoras. Neste caso, quando $B$ aumenta os LLs atravessam $\mu$ e a densidade $n_{2 D}$ varia. O outro caso é quando a depleção é total e a quantidade de elétrons confinada é constante. Neste caso, $\mu$ percorre os níveis dos estados localizados e estendidos dos LLs. Como $\mu$ varia e $n_{2 D}$ é constante nos casos estudados nesta tese, eu preciso inverter numericamente a Eq. (4.61) para obter o valor de $\mu$ para cada campo magnético.
} 


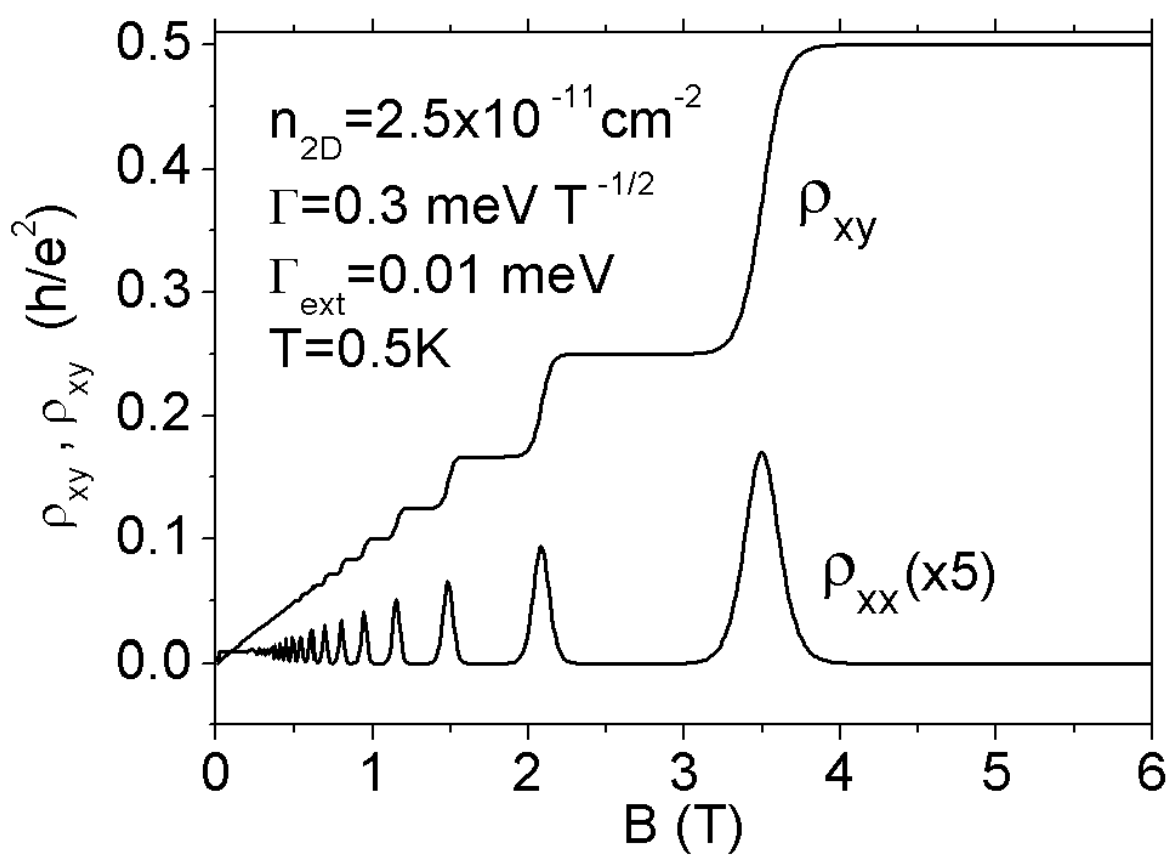

Figura 4.10: Resistividades longitudinal $\rho_{x x}$ e transversal $\rho_{x y}$ de um gás de elétrons bidimensional não-interagente e não-magnético. As curvas são características do regime de efeito Hall quântico inteiro, com a formação de platôs em $\rho_{x y}$ com resistividades que são submúltiplos de $h / e^{2}$. Já $\rho_{x x}$ apresenta as oscilações de Shubnikov-de Haas e regiões de resistividade nula correspondendo às regiões dos platôs. Esta simulação de magnetotransporte é para o sistema cuja estrutura de níveis de Landau é ilustrada na Fig. 4.6(a).

elétrons fosse estendida [122].

Portanto, para calcular o magnetotransporte no regime do efeito Hall quântico eu a utilizo no modelo de Ando e Uemura [118], mas substituindo a DOS total pela DOS $g_{\text {ext }}(\varepsilon)$ com alargamento gaussiano. Desta forma, as equações para as magnetocondutividades são [118-122, 132]

$$
\begin{gathered}
\sigma_{x x}=\frac{e^{2}}{h} \frac{2}{\pi} \int_{-\infty}^{\infty}\left(-\frac{\partial f(\varepsilon)}{\partial \varepsilon}\right) \sum_{i, n, \sigma_{z}}\left(n+\frac{1}{2}\right) \exp \left[-\left(\frac{\varepsilon-\varepsilon_{i, n}^{\sigma_{z}}}{\Gamma_{\text {ext }}^{\sigma_{z}}}\right)^{2}\right] \mathrm{d} \varepsilon \\
\sigma_{x y}=\frac{e n_{2 D}\left[g_{\text {ext }}(\varepsilon)\right]}{B}+\Delta \sigma_{x y},
\end{gathered}
$$

com $n_{2 D}\left[g_{\text {ext }}(\varepsilon)\right]$ a densidade bidimensional calculada usando a Eq. (4.61) mas com a DOS $g_{\text {ext }}(\varepsilon)$, Eq. (4.73), e

$$
\Delta \sigma_{x y}=\frac{e^{2}}{h} \frac{2}{\pi} \int_{-\infty}^{\infty}\left(-\frac{\partial f(\varepsilon)}{\partial \varepsilon}\right) \sum_{i, n, \sigma_{z}}\left(n+\frac{1}{2}\right) \frac{\Gamma_{\mathrm{ext}}^{\sigma_{z}}}{\hbar \omega_{c}} \exp \left[-\left(\frac{\varepsilon-\varepsilon_{i, n}^{\sigma_{z}}}{2 \Gamma_{\mathrm{ext}}^{\sigma_{z}}}\right)^{2}\right] \mathrm{d} \varepsilon
$$


As resistividades são obtidas simplesmente invertendo o tensor de condutividade

$$
\begin{aligned}
& \rho_{x x}=\frac{\sigma_{x x}}{\sigma_{x x}^{2}+\sigma_{x y}^{2}}, \\
& \rho_{x y}=\frac{\sigma_{x y}}{\sigma_{x x}^{2}+\sigma_{x y}^{2}} .
\end{aligned}
$$

A Fig. 4.10 exemplifica as curvas de magnetotransporte que são obtidas usado o modelo das Eqs. (4.73) a (4.76). Estas curvas são para o sistema simplificado usado nas ilustrações da Fig. 4.6, ou seja, um 2DEG não interagente e não magnético. Enquanto a largura da DOS total é e $\Gamma=0.3 \mathrm{meVT}^{-1 / 2}$, a largura para a DOS estendida é de $\Gamma_{\text {ext }}=0.01 \mathrm{meV}$.

As Eqs. (4.74a) e (4.75) são para o caso de espalhadores de curto alcance. Num caso mais geral, os termos $(n+1 / 2)$ que aparecem nas duas equações seriam escritos como $\left(\Gamma_{x x}^{i, n, \sigma_{z}} / \Gamma^{i, n, \sigma_{z}}\right)$ e $\left(\Gamma_{x y}^{i, n, \sigma_{z}} / \Gamma^{i, n, \sigma_{z}}\right)^{4}$, respectivamente [118-122, 132]. Neste caso, o alargamento de $g(\varepsilon)$ seria $\Gamma^{i, n, \sigma_{z}}$, variando para cada sub-banda $i$, nível de Landau $n$ e spin $\sigma_{z}$. Os termos $\Gamma_{x x}^{i, n, \sigma_{z}}$ e $\Gamma_{x y}^{i, n, \sigma_{z}}$ são funções do potencial de espalhamento e também teriam valores diferentes para cada nível com energia $\varepsilon_{i, n}^{\sigma_{z}}$. O efeito prático destes termos é determinar a amplitude das condutividades. Como no estudo que faço nos capítulos subsequentes eu estou interessado nos valores de campos magnéticos em que ocorrem os picos de $\rho_{x x}$, e na dependência com a temperatura, optei por não tentar simular a magnitude exata de cada pico. Desta forma, a versão com espalhadores de curto alcance do modelo para a magnetocondutividade é adequada para os propósitos dos próximos capítulos.

\subsection{Discussões e resumo da formulação}

Neste capítulo eu estendi a formulação utilizada até o Cap. 3 para o caso de sistemas dopados submetidos a altos campos magnéticos. As impurezas doadoras fornecem elétrons que são confinados no poço quântico formando um gás de elétrons bidimensional. A presença de um campo magnético intenso acarreta a quantização do movimento orbital nos chamados níveis de Landau. Neste regime, vários efeitos de natureza quântica se manifestam. Uma vez que os experimentos que eu analiso nos capítulos seguintes focalizam no magnetotransporte no regime do efeito Hall quântico inteiro, aqui eu apresentei um modelo teórico para calcular estas grandezas.

Nós dois próximos capítulos, nos quais eu investigo resultados experimentais em poços quânticos dopados do grupo II-VI, eu uso o modelo para 
sistemas dopados por modulação abordado neste capítulo em conjunto com o modelo para heteroestruturas digitais magnéticas dos capítulos anteriores. $\mathrm{Na}$ prática, os resultados das discussões nos Caps. 2 e 3 são incorporados no cálculo da estrutura eletrônica via o potencial dependente de spin, que modula o perfil da banda de condução. Assim, o potencial efetivo que utilizo na formulação de Kohn-Sham é

$v_{\text {eff }}^{\sigma_{z}}\left(z ; B, T,\left[n_{\uparrow}, n_{\downarrow}\right]\right)=\underbrace{v_{0}(z)+v_{b}(z)}_{\text {Estrutural }}+\underbrace{v_{\text {sp-d }}^{\sigma_{z}}(z ; B, T)}_{\text {Troca sp-d }}+\underbrace{v_{h}(z ;[n])}_{\text {Hartree }}+\underbrace{v_{x z}^{\sigma_{z}}\left(z ;\left[n_{\uparrow}, n_{\downarrow}\right]\right)}_{\text {Troca-correlação }}$.

Com a dependência espacial apenas na direção $\hat{z}$, o problema resume-se a resolver o hamiltoniano longitudinal unidimensional, $H_{\|}$na Eq. (4.47), para determinar as energias de sub-banda $\varepsilon_{i}^{\sigma_{z}}$ e as correspondentes funções envelope $\chi_{i}^{\sigma_{z}}(z)$. O espectro KS com as energias monoeletrônicas do sistema fica [Eq. (4.49)]

$$
\varepsilon_{i, n}^{\sigma_{z}}=\varepsilon_{i}^{\sigma_{z}}+\left(n+\frac{1}{2}\right) \hbar \omega_{c}+\frac{1}{2} g \mu_{B} \sigma_{z} B
$$

À partir deste espectro eu posso calcular a densidade de estados, densidades de portadores, magnetotransporte e outros observáveis de interesse teórico e experimental.

Para finalizar o capítulo eu resumo as principais equações do modelo no Quadro (2). 


\section{Quadro 2: Equações de trabalho.}

Equações autoconsistentes:

$$
\left[-\frac{\hbar^{2}}{2 m} \frac{\partial^{2}}{\partial z^{2}}+v_{e f f}^{\sigma_{z}}(z ; B, T)\right] \chi_{i}^{\sigma_{z}}(z)=\varepsilon_{i}^{\sigma_{z}} \chi_{i}^{\sigma_{z}}(z) \quad, \quad \nabla^{2} v_{h}(z)=-\frac{e^{2}}{\epsilon}\left[n(z)+n_{e}(z)-n_{i}(z)\right]
$$

com $n_{e}(z)$ a densidade de elétrons não depletados e $n_{i}(z)$ a densidade de doadores.

Potencial efetivo:

$$
v_{\text {eff }}^{\sigma_{z}}(z ; B, T)=\underbrace{v_{0}(z)+v_{b}(z)}_{\text {Estrutural }}+\underbrace{v_{\text {sp-d }}^{\sigma_{z}}(z ; B, T)}_{\text {Troca sp-d }}+\underbrace{v_{h}(z ;[n])}_{\text {Hartree }}+\underbrace{v_{x c}^{\sigma_{z}}\left(z ;\left[n_{\uparrow}, n_{\downarrow}\right]\right)}_{\text {Troca e correlação }}
$$

Espectro de energia:

$$
\varepsilon_{i, n}^{\sigma_{z}}=\varepsilon_{i}^{\sigma_{z}}+\left(n+\frac{1}{2}\right) \hbar \omega_{c}+\frac{1}{2} g \mu_{B} \sigma_{z} B
$$

Densidade de estados total com alargamento gaussiano:

$$
g(\varepsilon ; B, T)=\sum_{i, n, \sigma_{z}} g_{i, n}^{\sigma_{z}}(\varepsilon ; B, T)=\frac{e B}{h} \sum_{i, n, \sigma_{z}} \frac{1}{\Gamma \sqrt{2 \pi}} \exp \left[-\frac{\left(\varepsilon-\varepsilon_{i, n}^{\sigma_{z}}\right)^{2}}{2 \Gamma^{\sigma_{z}}}\right]
$$

Função de Fermi:

$$
f(\varepsilon ; \mu, T)=\frac{1}{1+\exp \left[(\varepsilon-\mu) / k_{B} T\right]}
$$

Perfil de densidade de elétrons confinados:

$$
n(z)=\sum_{i, n, \sigma_{z}}\left|\chi_{i, n}^{\sigma_{z}}(z)\right|^{2} \int_{-\infty}^{\infty} g_{i, n}^{\sigma_{z}}(\varepsilon) f(\varepsilon) \mathrm{d} \varepsilon
$$

Densidade bidimensional:

$$
n_{2 D}=\int_{-\infty}^{\infty} g(\varepsilon ; B, T) f(\varepsilon ; \mu, T) \mathrm{d} \varepsilon
$$

Energia total:

$$
E\left[n_{\uparrow}, n_{\downarrow}\right]=\int \varepsilon g(\varepsilon) f(\varepsilon) \mathrm{d} \varepsilon-\frac{1}{2} \int v_{h}(z) n(z) \mathrm{d} z-\sum_{\sigma_{z}} \int v_{x c}^{\sigma_{z}}(z) n_{\sigma_{z}}(z) \mathrm{d} z+E_{x c}\left[n_{\uparrow}, n_{\downarrow}\right]-T S_{s}\left[n_{\uparrow}, n_{\downarrow}\right]
$$

Magnetização:

$$
M=-\frac{\partial E}{\partial B}
$$

Magnetotransporte:

$$
\begin{gathered}
\sigma_{x x}=\frac{e^{2}}{h} \frac{2}{\pi} \int_{-\infty}^{\infty}-\frac{\partial f(\varepsilon)}{\partial \varepsilon} \sum_{i, n, \sigma_{z}}(n+1 / 2) \exp \left[-\left(\frac{\varepsilon-\varepsilon_{i, n}^{\sigma_{z}}}{\Gamma_{\mathrm{ext}}^{\sigma_{z}}}\right)^{2}\right] \mathrm{d} \varepsilon \\
\sigma_{x y}=\frac{e n_{2 D}\left[g_{e x t}(\varepsilon)\right]}{B}+\frac{e^{2}}{h} \frac{2}{\pi} \int_{-\infty}^{\infty}\left(-\frac{\partial f(\varepsilon)}{\partial \varepsilon}\right) \sum_{i, n, \sigma_{z}}\left(n+\frac{1}{2}\right) \frac{\Gamma_{\mathrm{ext}}^{\sigma_{z}}}{\hbar \omega_{c}} \exp \left[-\left(\frac{\varepsilon-\varepsilon_{i, n}^{\sigma_{z}}}{2 \Gamma_{\mathrm{ext}}^{\sigma_{z}}}\right)^{2}\right] \mathrm{d} \varepsilon \\
\rho_{x x}=\frac{\sigma_{x x}}{\sigma_{x x}^{2}+\sigma_{x y}^{2}} \quad, \quad \rho_{x y}=\frac{\sigma_{x y}}{\sigma_{x x}^{2}+\sigma_{x y}^{2}}
\end{gathered}
$$




\section{Capítulo 5}

\section{Dependência com a Temperatura das Oscilações de Shubnikov-de Haas}

\section{Conteúdo}

Cálculo da estrutura eletrônica, magnetotransporte, e energia total de um poço quântico digital magnético dopado por modulação, (Zn,Cd,Mn)Se. O cálculo reproduz os resultados experimentais de Knobel et al. [37] e Harris et al. [38], os quais investigam o aparecimento de um pico anômalo nas oscilações de Shubnikov-de Haas e um deslocamento para baixos campos das curvas de $\rho_{x x}$ à medida que a temperatura aumenta.

5.1 Introdução . . . . . . . . . . . . . . . . . . . . 110

5.2 Resumo dos resultados experimentais . . . . . . . . . . . . . 111

5.3 Resultados para magnetotransporte e energia total . . . . . . . 120

5.4 Discussões . . . . . . . . . . . . . . . . . . . . . 130 


\subsection{Introdução}

Heteroestruturas digitais magnéticas (DMHs) são excelentes laboratórios para se estudar gases de elétrons bidimensionais (2DEGs) no regime quântico. A interação de troca $s-d$ entre as impurezas magnéticas e os portadores acarreta um desdobramento de spin gigante em campos magnéticos relativamente baixos, como visto no Cap. 3. Já a técnica digital de inserção das impurezas magnéticas, aliada à técnica de dopagem por modulação, permite obter altos níveis de dopagem e uma alta mobilidade para os portadores $[8,12]$.

A magnitude das várias contribuições para a energia presentes nestes sistemas, a saber, o confinamento estrutural (2DEG), a quantização orbital (níveis de Landau, LL), a interação de troca sp-d (desdobramento Zeeman gigante), e os efeitos de muitos corpos (troca-correlação, XC), podem ser ajustados para compartilharem a mesma escala de energia. Os efeitos combinados destas interações acarretam uma física dependente de spin extremamente rica.

Os modelos apresentados nos Caps. 1 e 3 ignoraram a quantização de Landau e a interação de Coulomb. Já no Cap. 4 eu mostrei como incorporar estes ingredientes, no contexto da DFT/LSDA, para estudar gases de elétrons bidimensionais no regime de quantização de Landau. Para um caso mais simples, não-interagente e não-magnético, eu exemplifiquei como fica o diagrama de níveis de Landau, a energia total e a magnetização orbital, Fig. 4.6. Também apresentei o modelo de magnetotransporte que utilizo nesta tese para calcular as magnetoresistividades $\rho_{x x}$ e $\rho_{x y}$, ilustradas na Fig. 4.10 para o caso simplificado acima mencionado. Agora eu utilizo a mesma metodologia do Cap. 4, mais o modelo para DMHs do Cap. 1 e os modelos de perfis de concentração de Mn e de potencial dependente de spin dos Caps. 2 e 3, para estudar sistemas reais e que foram experimentalmente investigados.

Neste capítulo, eu investigo resultados experimentais de Knobel et al. [37] e Harris et al. [38] sobre o efeito da temperatura nas propriedades de magnetotransporte em um poço quântico digital magnético (DMQW) de (Zn,Cd,Mn)Se, dopado por modulação. O papel da temperatura é importante via dois mecanismos principais. Primeiro, através da função de Fermi, alargando a região em torno do potencial químico que contribui para os efeitos de transporte e, consequentemente, suavizando as curvas de magnetorresistividades em função do campo magnético. Segundo, a temperatura também é determinante na magnetização média dos íons de Mn, o que influencia diretamente a intensidade da interação de troca $s-d$.

As medidas de magnetotransporte no regime quântico [37, 38] mostraram que (i) as oscilações de Shubnikov-de Haas ( $\mathrm{SdH}$ ) deslocam para campos 
mais baixos à medida que a temperatura aumenta, e (ii) um pico anômalo de SdH surge no campo magnético $B_{c}=3.2 \mathrm{~T}$. O mesmo grupo experimental realizou uma análise fenomenológica destes resultados e concluiu que: o deslocamento das oscilações de SdH para baixos campos é devido ao efeito da temperatura na magnetização dos íons magnéticos, e que o pico anômalo ocorre quando há o cruzamento de dois níveis de Landau próximo ao nível de Fermi.

Entretanto, o modelo fenomenológico das Refs. [37, 38] não considera (i) o caracter autoconsistente da estrutura eletrônica de sistemas dopados por modulação, e (ii) a possível contribuição de efeitos de muitos corpos do 2DEG. Neste sentido, o modelo teórico descrito no Cap. 4 é mais abrangente, uma vez que leva estes pontos em consideração. De fato, usando este modelo não só foi possível reproduzir satisfatoriamente os resultados experimentais de magnetotransporte aqui mencionados, mas também reproduzir o aparecimento de ferromagnetismo de efeito Hall quântico em um sistema similar, o que apresento no próximo capítulo.

O corpo deste capítulo é dividido da seguinte forma. Primeiro, eu faço um resumo dos resultados experimentais e do modelo fenomenológico elaborado pelo grupo experimental para analisar os dados [37, 38]. Em seguida, eu apresento os resultados das minhas simulações, comparando com os resultados experimentais das Refs. [37, 38]. Eu finalizo o capítulo discutindo sobre a importância de se considerar o caracter autoconsistente da estrutura eletrônica e a relevância dos efeitos de muitos corpos nestas estruturas.

\subsection{Resumo dos resultados experimentais}

As referências $[37,38]$ apresentam medidas de magnetotransporte e magnetização em poços quânticos digitais magnéticos do grupo II-VI dopados por modulação. A heteroestrutura é semelhante àquela ilustrada na Fig. 4.1, pág. 74, com um poço quântico de largura $L=105 \AA$, espaçadores de $L_{S}=200 \AA$, e regiões $n$ dopadas de $L_{N}=120 \AA$. A Tabela 5.1 apresenta as principais características desta DMH.

O poço quântico contem frações de 1/16 monocamadas de MnSe "digitalmente" inseridas a cada 7 monocamadas de $\mathrm{Zn}_{1-y} \mathrm{Cd}_{y}$ Se. A concentração molar de Cd é de $y=0.13$, conferindo ao poço uma altura $v_{0} \sim 120 \mathrm{meV}{ }^{1}$ Este confinamento é suficiente para que o poço quântico capture todos os elétrons disponíveis na região dopada, de modo que a densidade do 2DEG permanece constante à medida que o campo magnético é variado. ${ }^{2}$ Medidas a baixo campo

\footnotetext{
${ }^{1}$ Usei o resultado de Liaci et al. [50] para o offset na banda de condução de poços quânticos de $\mathrm{ZnSe} / \mathrm{Zn}_{1-y} \mathrm{Cd}_{y} \mathrm{Se}$. O offset de $925 y \mathrm{meV}$ para a banda de condução foi o que melhor ajustou os dados experimentais para a energia de ligação de éxcitons.

${ }^{2}$ Medidas em amostras com $y=0.05$, que corresponde a um confinamento de $\sim 46 \mathrm{meV}$,
} 
Tabela 5.1: Características do poço quântico digital magnético de (Zn,Cd,Mn)Se das Refs. [37, 38]. Estão listadas as dimensões do poço e as concentrações de seus constituintes. Também são apresentadas as faixas de campo magnético e de temperatura usadas nos experimentos, bem como os resultados de algumas grandezas que caracterizam da amostra.

\begin{tabular}{|c|c|c|c|}
\hline Parâmetro & Símbolo & Valor & Observação \\
\hline \multicolumn{4}{|c|}{ Configuração do poço quântico digital magnético, Fig. 4.1} \\
\hline Largura do poço & $L$ & $105 \AA$ & \\
\hline Largura do espaçador & $L_{S}$ & $120 \AA$ & \\
\hline Largura da região dopada & $L_{N}$ & $200 \AA$ & \\
\hline Número de barreiras de MnSe & $N_{b}$ & 5 & \\
\hline Concentração planar de MnSe & $x_{p}$ & 0.0625 & \\
\hline Concentração molar de Cd & $y$ & 0.13 & \\
\hline \multicolumn{4}{|l|}{ Condições experimentais } \\
\hline Campo magnético & $B$ & $(0-8) \mathrm{T}$ & \\
\hline Temperatura & $T$ & $(0.33-7.0) \mathrm{K}$ & \\
\hline \multicolumn{4}{|l|}{ Caracterização da amostra } \\
\hline Densidade bidimensional & $n_{2 D}$ & $2.8 \times 10^{11} \mathrm{~cm}^{-2}$ & Baixo campo \\
\hline Mobilidade & $\mu$ & $0.8 \times 10^{4} \mathrm{~cm}^{2} / \mathrm{Vs}$ & Baixo campo \\
\hline Desdobramento de spin & $\Delta E$ & $31 \mathrm{meV}$ & \\
\hline
\end{tabular}

da densidade bidimensional resultaram em $n_{2 D}=(2.8 \pm 0.1) \times 10^{11} \mathrm{~cm}^{-2}$.

Efeito Hall quântico As medidas de magnetorresistência Hall $\rho_{x y}$, Fig. 5.1(a), apresentam desvios do regime linear clássico a partir de $\sim 0.8 \mathrm{~T}$. Observa-se a formação de platôs de resistividade característicos do regime de efeito Hall quântico, ${ }^{3}$ sendo o efeito melhor definido para $B \gtrsim 3.4 \mathrm{~T}$. Em particular, na região em torno de $B=3.0 \mathrm{~T}$ (circulo pontilhado), a curva de $\rho_{x y}$ apresenta um comportamento intrincado que sugere cruzamentos de níveis de Landau próximos do nível de Fermi. ${ }^{4}$ Note que $\rho_{x y}$ é linear com $B$ para campos baixos, indicando que a densidade $n_{2 D}$ não muda com o campo magnético.

resultaram em uma densidade bidimensional que varia em função de $B$ [137]. Neste caso, todos os níveis ligados do poço quântico são preenchidos e os elétrons que sobram permanecem na região dopada - que funciona como um reservatório de elétrons cujo nível das impurezas doadoras determina o potencial químico. À medida que um parâmetro externo é alterado, e.g. o campo magnético, a densidade $n_{2 D}$ confinada pode variar de maneira autoconsistente [138, 139]. Entretanto, este não é o caso dos sistemas investigados nesta tese.

${ }^{3}$ Os fatores de preenchimento associados aos platôs são inteiros, indicando o regime de efeito Hall quântico inteiro. Nesta amostra não foi observado o efeito fracionário.

${ }^{4}$ Cada platô numa curva de IQHE está associado a um determinado número de níveis de Landau abaixo do nível de Fermi (no platô $\rho_{x y}=h / \nu e^{2}$, com $\nu$ inteiro). As oscilações de $\rho_{x y}$ em torno de $B=3.0$ T na Fig. 5.1(a) sugerem que $\nu$ diminui, depois aumenta, e depois diminui novamente numa pequena faixa de campo magnético, sugerindo o cruzamento de níveis. 

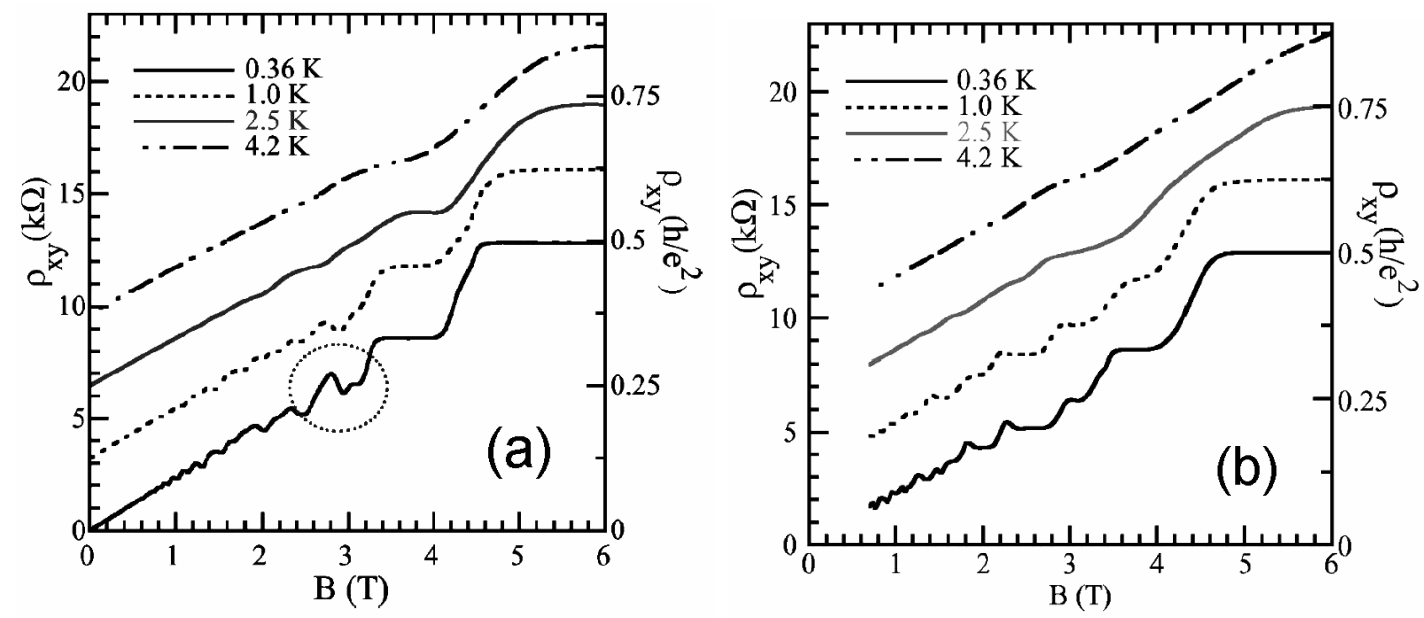

Figura 5.1: Resistividade Hall das DMHs de $n$-ZnSe/(Zn,Cd,Mn)Se para diversas temperaturas. Os painéis (a) e (b) apresentam os resultados experimentais e teóricos, respectivamente, de Knobel et al. [37]. O comportamento de $\rho_{x y}$ na região indicada no painel (a) sugere o cruzamento de níveis de Landau, como explicado no texto. Extraído de Knobel et al. [37].

Oscilações de Shubnikov-de Haas A Figura 5.2(a) mostra os resultados das medidas de magnetorresistividade $\rho_{x x}$. Note que nas mesmas regiões em que ocorrem platôs bem definidos de $\rho_{x y}$ na Fig. 5.1(a), aqui a resistividade $\rho_{x x}$ fica praticamente nula, outra característica do regime de efeito Hall quântico. Aqui eu estou particularmente interessado em dois aspectos destes resultados.

1. A $T=0.35 \mathrm{~K}$ ocorre um pico anômalo no gráfico de $\mathrm{SdH}$ em $B_{c}=3.2 \mathrm{~T}$ (veja as setas), na mesma região de campo magnético das oscilações de $\rho_{x y}$ na Fig. 5.1(a). Para $T=1.0 \mathrm{~K}$ esta oscilação é atenuada, não mais aparecendo em temperaturas maiores.

2. À medida que $T$ aumenta, além da atenuação das oscilações, ocorre um deslocamento dos picos de SdH para campos magnéticos mais baixos.

Efeito de Haas-van Alphen As magnetizações apresentadas na Fig. 5.3(c) foram medidas em um magnetômetro micromecânico ultrasensível [38, 140], Fig. 5.3(a). A medida é baseada no deslocamento $\Delta \nu_{0}(B)$ da frequência de ressonância da vibração da haste que sustenta a amostra, resultado que eu reproduzo na Fig. 5.3(b). Harris et al. [38] entenderam que a curva de fundo (envelope) é oriunda apenas da magnetização dos íons de Mn, e que as pequenas oscilações correspondem ao sinal de dHvA do 2DEG. Assim, eles modelaram a curva de fundo para separar a contribuição do Mn [140, 141] e subtraíram o resultado dos dados experimentais. Com isso, restou apenas a parte oscilatória, que é relacionada com a magnetização usando a expressão $M=\Delta \nu_{0}(B) / 2 B \gamma \nu_{0}(0)$, 

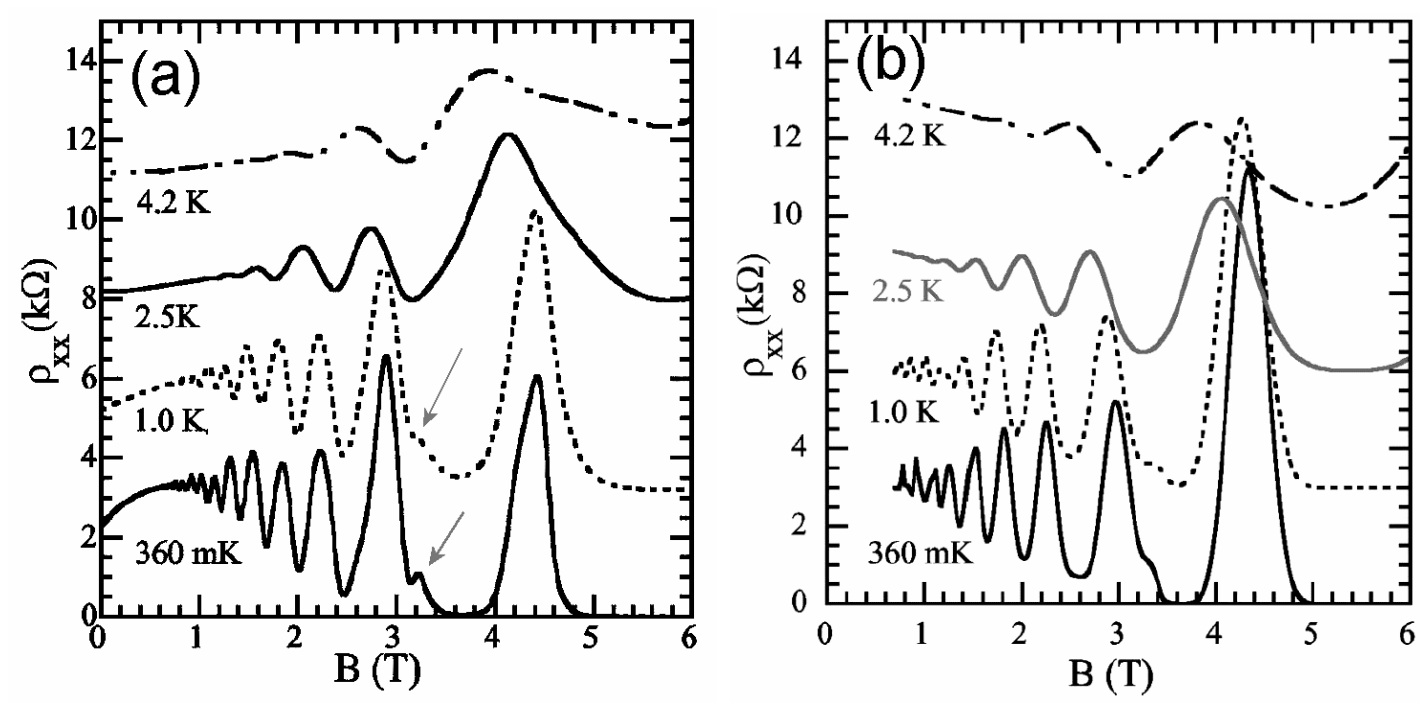

Figura 5.2: Oscilações de Shubnikov-de Haas das DMHs de $n$ $\mathrm{ZnSe} /(\mathrm{Zn}, \mathrm{Cd}, \mathrm{Mn}) \mathrm{Se}$ para diversas temperaturas. Em (a) estão os resultados experimentais de Knobel et al. [37]. Note (i) o surgimento de um pico anômalo em $B_{c}=3.2 \mathrm{~T}$ (setas), e (ii) o deslocamento dos picos para baixos campos à medida que $T$ aumenta. O resultado da simulação da Ref. [37] é apresentado em (b). Extraído de Knobel et al. [37].

sendo $\gamma$ uma constante de torção da haste do magnetômetro e $\nu_{0}(0)$ a frequência vibracional a campo magnético nulo [142].

As oscilações foram detectadas para $B \gtrsim 1 \mathrm{~T}$. Elas deslocam-se para campos magnéticos menores e diminuem de intensidade com o aumento da temperatura, como no caso de $\rho_{x x}$. Em $B \sim 3.2 \mathrm{~T}$, a curva para $T=0.32 \mathrm{~K}$ tem uma pequena descontinuidade. Isto deve ocorrer devido a uma alteração abrupta de população dos LLs próximos ao nível de Fermi, reforçando a hipótese levantada para o cruzamento de níveis nesta faixa de campo magnético. Adicionalmente, ao contrastar estas oscilações de dHvA com o comportamento do tipo "dente de serra" de 2DEGs em amostras de GaAs com alta mobilidade [e.g. 143, 144], a relativa suavidade das oscilações indica que o alargamento dos níveis de Landau devido a desordem deve ser comparável com a separação entre eles [38].

Não periodicidade das oscilações quânticas A desordem mencionada no parágrafo anterior também reflete na periodicidade das oscilações de dHvA e SdH. Normalmente, os mínimos de SdH ocorrem quando o fator de preenchimento é inteiro. Na Fig. 5.4, na qual $\rho_{x x}$ é apresentado em função de $1 / B$, nota-se que este não é o caso. Para $\nu \leq 7$ há um mínimo de $\mathrm{SdH}$ para cada valor inteiro de $\nu$; já para $7<\nu \leq 14$ há 5 mínimos para 7 valores de $\nu$. Provavelmente, para campos altos $(\nu \leq 7)$, os LLs são bem resolvidos. Já para campos mais baixos $(\nu>7)$, há sobreposição destes níveis, consequência do alargamento 

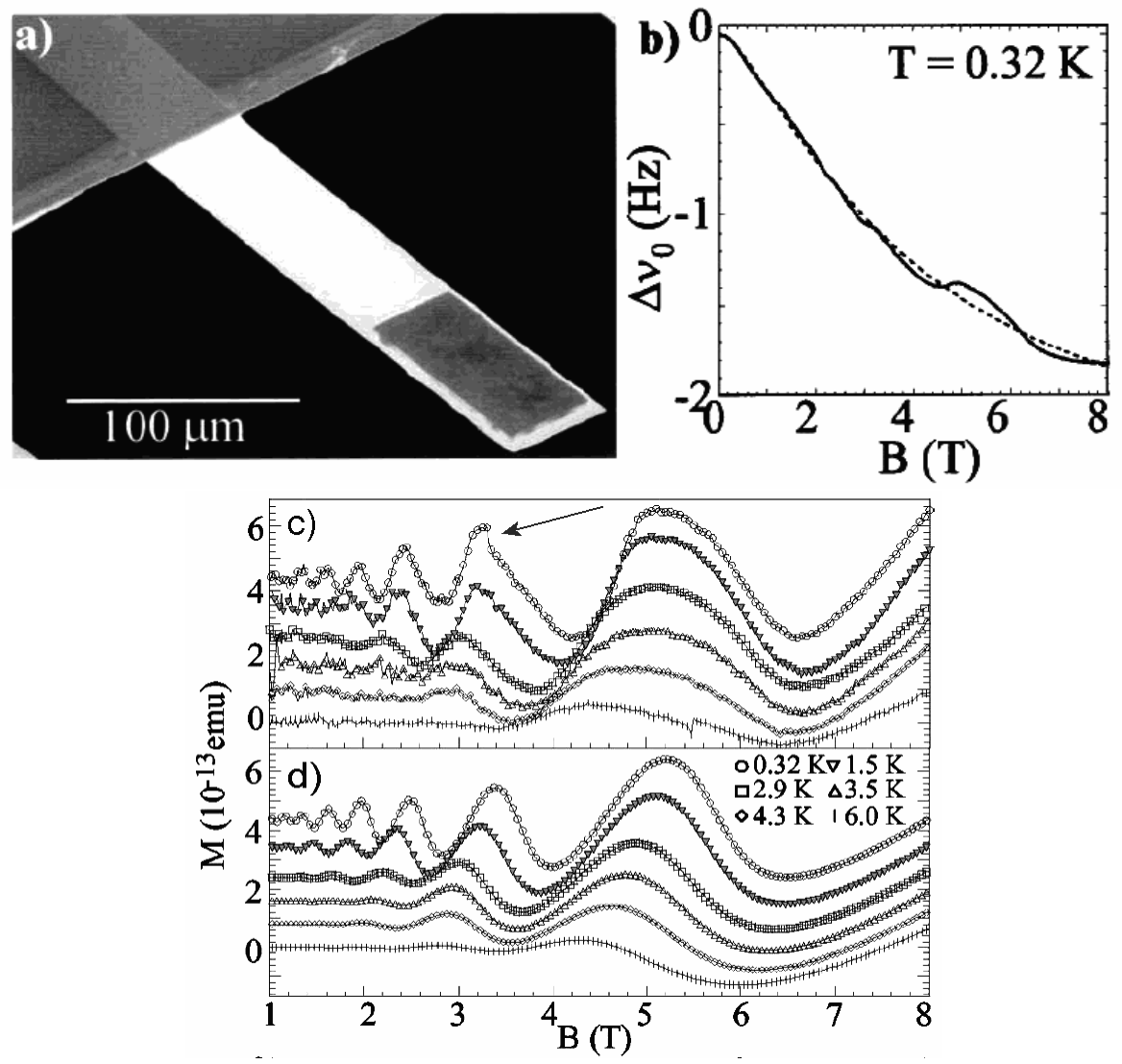

Figura 5.3: Oscilações de de Haas-van Alphen na magnetização das amostras de $n$-ZnSe/(Zn,Cd,Mn)Se de Knobel et al. [37] e Harris et al. [38]. Para efetuar estas medidas, a amostra fica na extremidade da haste de um micromagnetômetro (a), cujas vibrações são detectadas com o auxílio de um laser. A grandeza medida é o desvio da frequência fundamental de oscilação da haste em função do campo magnético (b). A curva "envelope" em (b) foi interpretada como resultante da magnetização do Mn, e as oscilações em torno dela como provenientes dos elétrons de condução. Em (c) estão os resultados experimentais para a magnetização para várias temperaturas e em (d) as simulações realizadas pelo grupo experimental. Extraído de Harris et al. [38], (a) e (b), e de Knobel et al. [37], (c) e (d).

dos LLs devido a desordem $\left(\hbar \omega_{c}<\Gamma\right)$. Esta aperiodicidade também ocorre nas medidas de dHvA [37, 38].

\subsubsection{Modelo fenomenológico do grupo experimental}

Para entender estes resultados, Knobel et al. [37] e Harris et al. [38] elaboraram um modelo fenomenológico para os níveis de energia e densidade de estados do sistema. Usando este modelo, eles simularam o magnetotransporte e a magnetização do 2DEG. Nos parágrafos seguintes eu reproduzo este modelo e os respectivos resultados, justamente com o intuito de contrastar com o modelo autoconsistente que apresentei no Cap. 4 e com os meus resultados que apresento na próxima seção. 


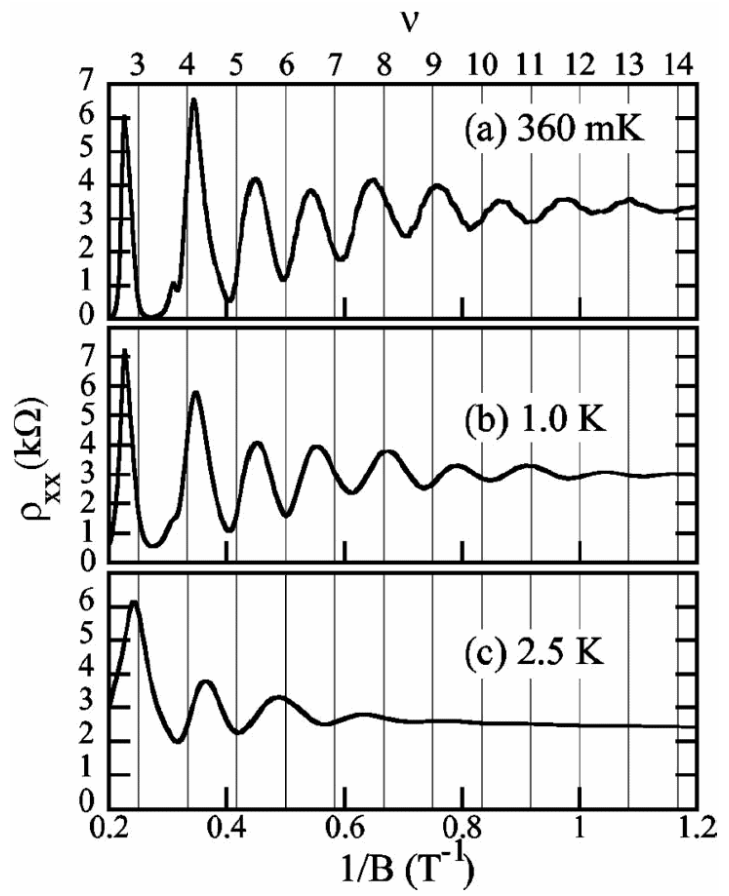

Figura 5.4: Oscilações de Shubnikov-de Haas da Fig. 5.1(a) são apresentadas em função de $1 / B$. O fator de preenchimento $\nu=h n_{2 D} / e B$, calculado usando $n_{2 D}=$ $2.9 \times 10^{11} \mathrm{~cm}^{-2}$, é indicado na abcissa superior. As oscilações são aperiódicas com $1 / B$, contrastando com o caso usual em que os mínimos de $\rho_{x x}$ ocorrem para valores inteiros de $\nu$. Extraído de Knobel et al. [37].

Espectro de Landau e DOS A energia de cada nível de Landau $n=0,1,2, \ldots$ com spin $\sigma_{z}= \pm 1$ foi escrita como a soma da energia de cíclotron e a contribuição da interação de troca $s-d$ para o desdobramento de spin,

$$
\varepsilon_{n}^{\sigma_{z}}=\left(n+\frac{1}{2}\right) \hbar \omega_{c}+\sigma_{z} \frac{1}{2} \Delta E_{S_{\max }} B_{5 / 2}\left(\frac{5 \mu_{B}}{k_{B}\left(T+T_{0}\right)}\right),
$$

sendo $B_{5 / 2}$ a função de Brillouin, $T$ a temperatura e $T_{0}$ uma temperatura efetiva. $\Delta E_{S_{\max }}$ obtido experimentalmente por medidas de fotoluminescência, é o valor de saturação do desdobramento de spin de elétron. Este é um dos principais parâmetros do modelo do grupo experimental. Ele indica a magnitude do desdobramento de spin e, consequentemente, determina a posição em que ocorrem cruzamentos de níveis de Landau. A Fig. 5.5 ilustra os níveis de Landau calculados usando a Eq. (5.1).

O modelo supõe que o número de elétrons no poço é sempre constante, e pode ser calculado integrando a densidade de estados total $g(\varepsilon, B, T)$ juntamente com a função de Fermi $f(\varepsilon, T)$

$$
n_{2 D}=\int_{-\infty}^{\infty} g(\varepsilon, B, T) f(\varepsilon, T) \mathrm{d} \varepsilon
$$



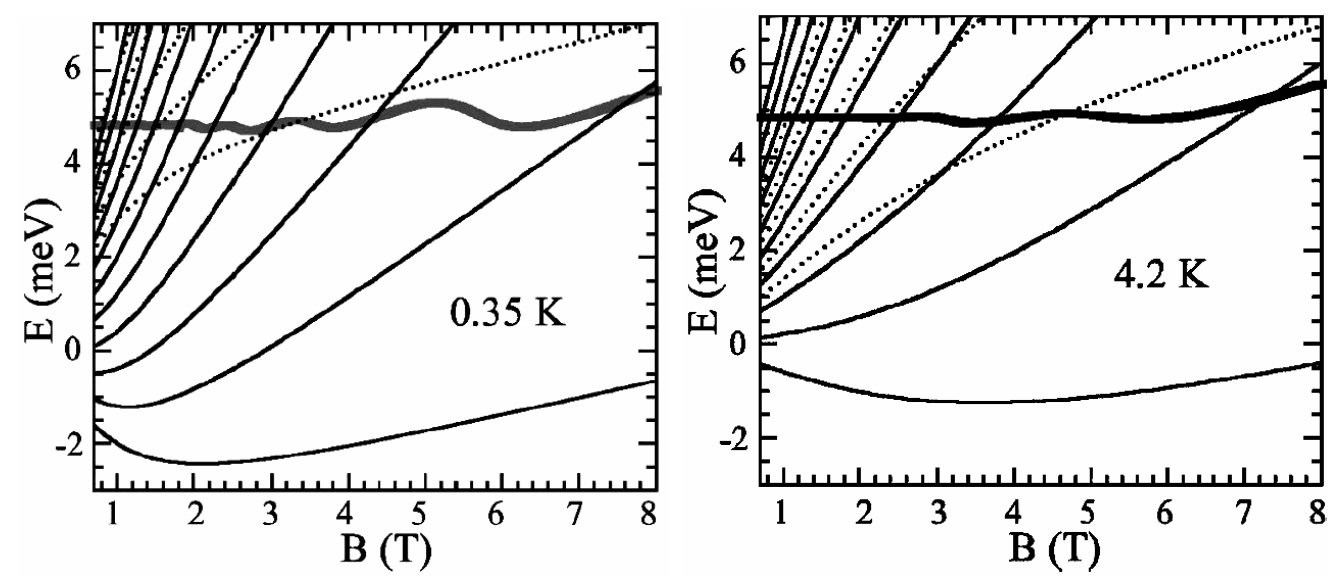

Figura 5.5: Diagramas de níveis de Landau, a $T=0.35 \mathrm{~K}$ e $T=4.2 \mathrm{~K}$, utilizados na Ref. [37] nas simulações de magnetotransporte e magnetização.

A DOS $g(\varepsilon, B, T)$ é modelada usando os níveis da Eq. (5.1) com alargamento $\Gamma(B)$ devido a desordem. O modelo de DOS que retornou melhores resultados foi com alargamento gaussiano, ${ }^{5}$ semelhante à Eq. (4.54) na pág. 95, com largura característica $\Gamma(B)=0.36 B^{1 / 2} \mathrm{meVT}^{-1 / 2}$.

Energia livre e oscilações de $\operatorname{dHvA}$ A simulação para a magnetização foi efetuada calculando a primeira derivada de energia livre com relação ao campo magnético. O modelo usou a expressão da energia livre de Helmholtz de um gás de elétrons uniforme não-interagente [114, Cap. 2, Problema 2]

$$
F=n_{2 D} \mu-k_{B} T \int_{\infty}^{\infty} g(\varepsilon, B, T) \ln \left[1+\exp \left(\frac{\mu-\varepsilon}{k_{B} T}\right)\right] \mathrm{d} \varepsilon
$$

Os parâmetros utilizados foram $\Delta E_{S_{\max }}=7.7 \mathrm{meV}, T_{0}=1.55 \mathrm{~K}$, e $n_{2 D}=2.94 \times$ $10^{11} \mathrm{~cm}^{-2}$, além do alargamento $\Gamma(B)$ na DOS. Note que, uma vez que a densidade $n_{2 D}$ é fixa, o potencial químico é calculado invertendo-se a Eq. (5.2). Os resultados destas simulações estão apresentados na Fig. 5.3(d). As posições das oscilações e o deslocamento com a temperatura concordam com os experimentos. Já o formato das curvas e detalhes, como a pequena descontinuidade em $B \sim 3.2 \mathrm{~T}$ a $T=0.35 \mathrm{~K}$, não são tão bem reproduzidos.

Magnetotransporte Para simular o magnetotransporte, Knobel et al. [37] e Harris et al. [38] utilizaram um modelo similar àquele que apresentei no Cap. 4, baseado na visão de Aoki e Ando [117] e Prange [136] para o IQHE. O transporte se dá quando o nível de Fermi passa pelos estados extendidos no centro de cada nível de Landau (veja a Fig. 4.8 na pág. 102). O perfil utilizado

\footnotetext{
${ }^{5}$ Knobel [145] também testou alargamentos lorentzianos, mas os resultados foram inferiores.
} 
para a DOS extendidos $g_{e x t}^{n, \sigma_{z}}(\varepsilon, B, T)$ é gaussiano, e possui largura característica $\Gamma_{e x t}^{\sigma_{z}}$, independente de $B$ e diferente para cada canal de spin.

As equações usadas para as resistividades de cada canal de spin são

$$
\begin{gathered}
\rho_{x x}^{\sigma_{z}}(B, T)=\rho_{0} \int_{-\infty}^{\infty} \sum_{n} g_{e x t}^{n, \sigma_{z}}(\varepsilon, B, T) \frac{\partial f(\varepsilon, T)}{\partial \varepsilon} \mathrm{d} \varepsilon, \\
\rho_{x y}^{\sigma_{z}}(B, T)=\frac{B}{e \int_{-\infty}^{\infty} \sum_{n} g_{e x t}^{n, \sigma_{z}}(\varepsilon, B, T) f(\varepsilon, T) \mathrm{d} \varepsilon} .
\end{gathered}
$$

O prefator $\rho_{0}$ é um parâmetro usado para ajustar a amplitude das oscilações de $\mathrm{SdH}$ com os dados experimentais. Para obter as resistividades totais são efetuados os seguintes passos: (1) calcula-se as resistividades de cada canal de spin usando (5.4), com as quais (2) calcula-se as condutividades de cada canal de spin $\sigma_{z}$,

$$
\sigma_{x x}^{\sigma_{z}}=\frac{\rho_{x x}^{\sigma_{z}}}{\rho_{x x}^{\sigma_{z}}+\rho_{x y}^{\sigma_{z}^{2}}} \quad, \quad \sigma_{x y}^{\sigma_{z}}=\frac{\rho_{x y}^{\sigma_{z}}}{\rho_{x x}^{\sigma_{z}^{2}}+\rho_{x y}^{\sigma_{z}^{2}}}
$$

(3) Em seguida obtém-se as condutividades totais,

$$
\sigma_{x x}=\sigma_{x x}^{\uparrow}+\sigma_{x x}^{\downarrow} \quad, \quad \sigma_{x y}=\sigma_{x y}^{\uparrow}+\sigma_{x y}^{\downarrow}
$$

e (4) inverte-se novamente o tensor de condutividade para obter a resistividade total,

$$
\rho_{x x}=\frac{\sigma_{x x}}{\sigma_{x x}^{2}+\sigma_{x y}^{2}} \quad, \quad \rho_{x y}=\frac{\sigma_{x y}}{\sigma_{x x}^{2}+\sigma_{x y}^{2}} .
$$

Os parâmetros que melhor reproduziram os resultados de magnetotransporte foram: $\Delta E_{S_{\max }}=7.2 \mathrm{meV}, T_{0}=1.55 \mathrm{~K}, n=2.9 \times 10^{11} \mathrm{~cm}^{-2}$, $\Gamma=0.36 B^{-1 / 2} \mathrm{meV}, \Gamma_{e x t}^{\downarrow}=0.25 \mathrm{meV}$ e $\Gamma_{e x t}^{\uparrow}=0.05 \mathrm{meV}{ }^{6}$ A Ref. [37] não indica os valores usados para $\rho_{0} \cdot{ }^{7}$ Note que, apesar de $\Delta E_{S_{\max }}$ e $n_{2 D}$ serem ligeiramente inferiores àqueles usados nas simulações de $\mathrm{dHvA}$, o conjunto dos parâmetros das duas simulações ainda são consistentes com os valores experimentalmente medidos. Os resultados das simulações para $\rho_{x x}$ e $\rho_{x y}$ são reproduzidos nas Figs. 5.2(b) e $5.1(\mathrm{~b})$, respectivamente.

O modelo fenomenológico proposto pelo grupo experimental reproduz os resultados experimentais de maneira bastante satisfatória. Na resistividade longitudinal, Fig. 5.2, tanto o aparecimento do pico anômalo quanto o desloca-

\footnotetext{
${ }^{6}$ Segundo Knobel [145, Tese de doutorado], $\Gamma_{\text {ext }}^{\downarrow}=0.25 \mathrm{meV}$ e $\Gamma_{\text {ext }}^{\uparrow}=0.05 \mathrm{meV}$ apenas para $T=0.36 \mathrm{~K}$. Para temperaturas maiores $\Gamma_{\text {ext }}^{\uparrow}$ é maior, sendo $\Gamma_{\text {ext }}^{\uparrow}=\Gamma_{\text {ext }}^{\downarrow}$ para altas temperaturas. A referência não indica quais são os valores intermediários de $\Gamma_{\text {ext }}^{\uparrow}$.

${ }^{7}$ Diferente da Ref. [37], a Ref. [145, Tese de doutorado] indica que as simulações dos resultados de magnetotransporte usaram diferentes valores de $\rho_{0}$ para cada spin e para cada temperatura. Foram usados $\rho_{0}^{\uparrow}=0.12 \rho_{0}^{\downarrow}$ para $T=0.36 \mathrm{~K}$ e $1.0 \mathrm{~K}$, e $\rho_{0}^{\uparrow}=\rho_{0}^{\downarrow}$ para temperaturas maiores. Os valores numéricos não são indicados.
} 
mento para baixos campos à medida que a temperatura aumenta se manifestam. O deslocamento com a temperatura vem da dependência da função de Brillouin com $T$. A hipótese de cruzamento de LLs é corroborada pelo diagrama de níveis de Landau da Fig. 5.5 - em $B \sim 3.0 \mathrm{~T}$ os LLs $|3, \downarrow\rangle$ e $|0, \uparrow\rangle$ cruzam próximos do nível de Fermi. O pico anômalo surge porque o nível de Fermi cruza os estados estendidos de $|0, \uparrow\rangle$ em $B \sim 3.2 \mathrm{~T}$, cf. Fig. 5.5. Estes pontos indicam que os ingredientes que o modelo inclui são de fato os mais relevantes. São eles (i) a quantização de Landau, (ii) o desdobramento de spin gigante devido a interação de troca $s-d$, e (iii) uma densidade de estados com níveis estendidos e localizados, que simula a situação na qual apenas os estados estendidos contribuem para o magnetotransporte.

Apesar da concordância com os resultados experimentais, alguns pontos merecem ser levantados e, consequentemente, motivam a investigação teórica que eu apresento neste capítulo.

1. A análise fenomenológica baseia-se numa estrutura "rígida" para a estrutura eletrônica $\varepsilon_{n}^{\sigma_{z}}(B)$, Eq. (5.1), com uma componente linear com $B$ proveniente da frequência de cíclotron e outra que depende do campo via a função de Brillouin. Entretanto, o modelo não leva em consideração o carácter autoconsistente usual da estrutura eletrônica de sistemas dopados. Além disso, a magnitude do desdobramento de spin não é calculada, mas sim extraída de experimentos de fotoluminescência.

2. Os valores para a concentração nominal de $\operatorname{Mn} x_{p}=0.0625$ e o desdobramento de spin máximo $\Delta E_{\max }=7.2 \mathrm{meV}$ aparentam ser inconsistentes. Usando as definições (2.32) e (2.33) para concentração efetiva, o valor para o desdobramento de spin implica $x_{e f f}=0.01162$. Eu então utilizei a teoria para aglomeração e diluição do Cap. 2 para estimar qual deve ser a concentração nominal de Mn que fornece o desdobramento de spin obtido na Ref. [37]. Para um perfil de segregação com comprimento característico $\ell=5 \AA$ e modelo de aglomerados de singletos e tripletos, o valor experimental para $\Delta E_{\max }$ corresponde a uma concentração nominal de $x_{p}=0.096$, superior ao valor nominal experimental de $0.065 .{ }^{8}$ Admitindo que o modelo para aglomeração e diluição do Cap. 2 descreve satisfatoriamente o efeito das impurezas magnéticas, concluo que (i) a concentração nominal $x_{p}=0.0625$ não é suficiente para explicar a magnitude do desdobramento de spin observado, e consequentemente, (ii) deve existir alguma outra con-

\footnotetext{
${ }^{8}$ Note que o valor de $\ell$ aqui não é um fator importante pois $x_{e f f}$ varia pouco com ele para $x_{p}$ pequenos. Veja a Fig. 2.12. Assim, mesmo para valores de $\ell$ diferentes, ainda tenho um $x_{p}$ maior que o nominal.
} 
tribuição para o desdobramento de spin que não está no modelo proposto pelo grupo experimental.

3. O modelo para o magnetotransporte começa com $\rho_{x x}^{\sigma_{z}}$ para cada componente de spin, Eq. (5.4a). Depois calcula-se as componentes do tensor $\hat{\sigma}$ e invertese novamente para obter $\hat{\rho}$. Seria interessante um modelo que seguisse a linha teórica mais comum, i.e., a condutividade é a grandeza teoricamente investigada e, a partir dela, se calcula a resistividade, que é a grandeza experimental.

Motivado por estes três pontos, apliquei o modelo de DMHs que apresento no Capítulo 4 para estudar as propriedades do DMQW investigado nas Refs. [37, 38]. Os dois principais ingredientes adicionais que eu utilizo, quando comparado com o modelo proposto pelo grupo experimental, são a autoconsistência no cálculo da estrutura eletrônica e a inclusão do termo de troca-correlação dependente de spin. Na seção seguinte eu apresento um resumo da formulação teórica usada juntamente com os respectivos resultados.

\subsection{Resultados para magnetotransporte e energia total}

Nesta seção eu aplico o modelo teórico para 2DEGs confinados em DMHs, apresentado no Cap. 4, para estudar as propriedades do poço quântico digital magnético investigado experimentalmente nas Refs. [37, 38] - veja o Quadro 2, na página 108, para um resumo deste modelo.

Eu calculo a estrutura eletrônica do sistema e função do campo magnético e da temperatura. Em seguida, eu calculo as grandezas que foram experimentalmente investigadas, a saber, densidades, e magnetorresistividades $\rho_{x x} \mathrm{e}$ $\rho_{x y}$.

Os parâmetros utilizados nestas simulações são baseados nos dados das correspondentes amostras experimentais, Tabela 5.1, e em valores conhecidos na literatura para diversas propriedades dos materiais semicondutores usados na fabricação destas DMHs. Estes valores, bem como os valores para os parâmetros que ajustei para melhor reproduzir os dados experimentais, estão listados na Tabela 5.2.

Nos parágrafos seguintes eu apresento os resultados destas simulações e, na próxima seção, eu discuto estes resultados comparando-os com os achados experimentais. 
Tabela 5.2: Parâmetros do poço quântico digital magnético de (Zn,Cd,Mn)Se das Refs. [37, 38]. A primeira parte da tabela contem valores da literatura para várias propriedades dos materiais utilizados na DMH. A segunda parte lista os valores dos parâmetros que usei nas simulações.

\begin{tabular}{|c|c|c|c|}
\hline Parâmetro & Símbolo & Valor & Observação/Referência \\
\hline \multicolumn{4}{|c|}{ Dados relevantes sobre o material } \\
\hline Massa efetiva & $m / m_{0}$ & 0.145 & {$[146]$} \\
\hline Constante dielétrica estática & $\epsilon / \epsilon_{0}$ & 9.0 & {$[147]$} \\
\hline Parâmetro de rede & $a$ & 5.6676 & [148] \\
\hline Fator de Landé efetivo & $g$ & 1.2 & [149] \\
\hline Constante de troca $s-d$ & $N_{0} \alpha$ & $260 \mathrm{meV}$ & {$[150]$} \\
\hline gap $\mathrm{ZnSe}$ & $E_{\text {gap }}^{Z n S e}$ & $2.822 \mathrm{eV}$ & {$[151]$} \\
\hline gap CdSe & $E_{\text {gap }}^{C d S e}$ & $1.818 \mathrm{eV}$ & {$[52]$} \\
\hline gap $\mathrm{MnSe}$ & $E_{g a p}^{M n S e}$ & $3.5 \mathrm{eV}$ & {$[152]$} \\
\hline Offset banda condução & $C B O$ & 0.8 & {$[53]$} \\
\hline \multicolumn{4}{|l|}{ Parâmetros nas simulações } \\
\hline Offset na interface ZnSe/ZnCdSe & $v_{0}$ & $120 \mathrm{meV}$ & $925 y \mathrm{meV}[50]$ \\
\hline Offset na interface $\mathrm{ZnCdSe} / \mathrm{MnSe}$ & $v_{b}$ & $648 \mathrm{meV}$ & \\
\hline Densidade bidimensional & $n_{2 D}$ & $2.8 \times 10^{11} \mathrm{~cm}^{-2}$ & \\
\hline \multirow[t]{2}{*}{ Concentração planar de MnSe } & $x_{p}$ & 0.035 & LSDA \\
\hline & & 0.085 & Hartree \\
\hline Temperatura efetiva & $T_{0}$ & $0.55 \mathrm{~K}$ & \\
\hline Função de perfil para MnSe & $\wp(z)$ & $\wp_{\exp }(z)$ & Eq. (2.24) \\
\hline Comprimento de uma monocamada & $d$ ou ML & $3 \AA$ & $d \sim a / 2$ \\
\hline Comprimento característico & $\ell$ & $4.326 \AA$ & $1.44 \mathrm{ML}$ \\
\hline Alargamento DOS & $\Gamma$ & $0.38 \mathrm{meV}$ & \\
\hline \multirow[t]{2}{*}{ Alargamento DOS estados extendidos } & $\Gamma_{e x t}^{\uparrow}$ & $0.15 \mathrm{meV}$ & \\
\hline & $\Gamma_{e x t}^{\downarrow}$ & $0.25 \mathrm{meV}$ & \\
\hline
\end{tabular}

Perfil de potencial O primeiro passo para calcular a estrutura eletrônica do poço quântico é calcular o perfil de potencial a ser utilizado nas equações de Kohn-Sham. Este potencial efetivo, que inclui os termos estrutural, troca $s-d$, Hartree, e troca-correlação, é escrito como, Eq. (4.77),

$v_{\text {eff }}^{\sigma_{z}}\left(z ; B, T,\left[n_{\uparrow}, n_{\downarrow}\right]\right)=\underbrace{v_{0}(z)+v_{b}(z)}_{\text {Estrutural }}+\underbrace{v_{\mathrm{sp}-\mathrm{d}}^{\sigma_{z}}(z ; B, T)}_{\text {Troca sp-d }}+\underbrace{v_{h}(z ;[n])}_{\text {Hartree }}+\underbrace{v_{x c}^{\sigma_{z}}\left(z ;\left[n_{\uparrow}, n_{\downarrow}\right]\right)}_{\text {Troca-correlação }}$.

Naturalmente, os termos de Hartree e troca-correlação são recalculados a cada passo do procedimento autoconsistente. A Fig. 5.6 mostra o perfil de potencial final para elétrons com spin para baixo a $B=3 \mathrm{~T}$ e $T=0.36 \mathrm{~K}$.

Aqui eu optei por utilizar perfis de segregação (exponencial) para simular a diluição do $\mathrm{Mn}$ ao longo da amostra. ${ }^{9}$ Eu fixei o comprimento característico em $\ell=1.44 \mathrm{ML}$ e ajustei o valor da concentração $x_{p}$ de Mn para o

\footnotetext{
${ }^{9}$ Perfis de interdifusão também foram testados e fornecem resultados similares.
} 


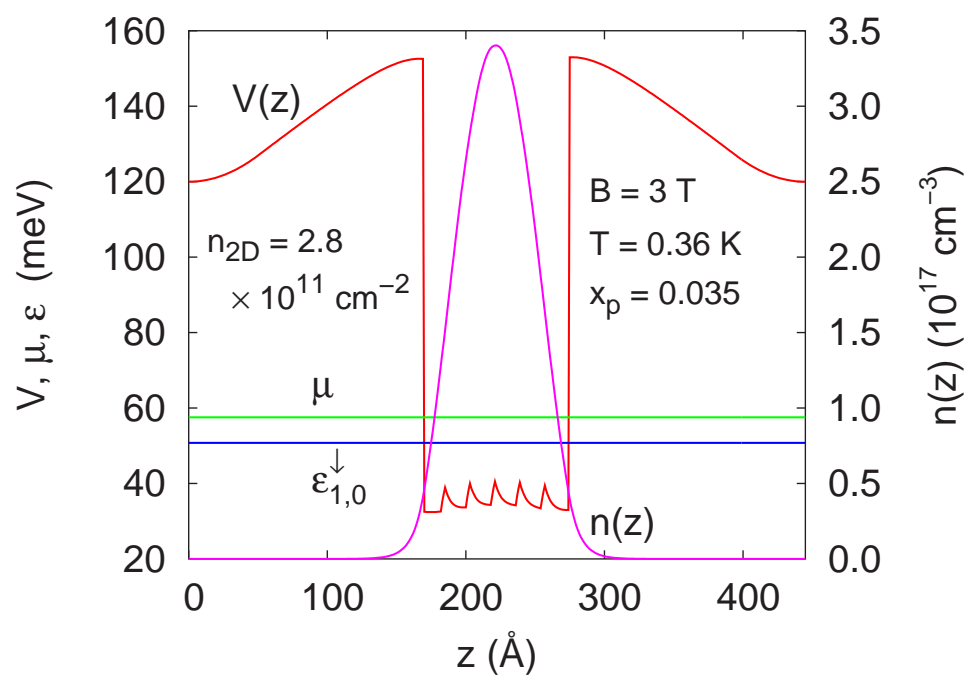

Figura 5.6: Perfil de potencial autoconsistente para elétrons com spin para baixo no poço quântico digital magnético dopado por modulação. A energia do nível $\varepsilon_{1,0}^{\downarrow}$ e do potencial químico $\mu$ também estão indicados. O perfil de densidade $n(z)$ mostra que o gás de elétrons está bastante confinado, penetrando pouco nas barreiras laterais do poço quântico. As camadas magnéticas foram modeladas usando um perfil de concentração típico de segregação (perfil exponencial).

valor que melhor reproduziu os resultados experimentais. ${ }^{10}$ Para a aglomeração antiferromagnética eu considerei o modelo que inclui singletos e tripletos - veja a Fig. 2.8, na página 36. Este foi o modelo que apresentou melhores resultados no Cap. 3.

Diagrama de níveis de Landau A estrutura eletrônica é então calculada resolvendo as equações autoconsistentes de Kohn-Sham - equação de Schrödinger mais interação de Coulomb - para os elétrons na banda de condução, Eq. (4.19),

$$
\left[-\frac{\hbar}{2 m} \frac{\partial^{2}}{\partial z^{2}}+v_{e f f}^{\sigma_{x}}\left(z ; B, T,\left[n_{\uparrow}, n_{\downarrow}\right]\right)\right] \chi_{i}^{\sigma_{z}}(z)=\varepsilon_{i}^{\sigma_{z}} \chi_{i}^{\sigma_{z}}(z) \text {. }
$$

A energia de cada nível de Landau consiste da energia da sub-banda, da energia de cíclotron, e da energia Zeeman ordinária. Ela é escrita como, Eq. (4.49),

$$
\varepsilon_{i, n}^{\sigma_{z}}=\varepsilon_{i}^{\sigma_{z}}+\left(n+\frac{1}{2}\right) \hbar \omega_{c}+\frac{\sigma_{z}}{2} g^{*} \mu_{B} B
$$

\footnotetext{
${ }^{10}$ Como discutido no Cap. 2, para baixas concentrações $x_{p}$, a concentração efetiva, e consequentemente o desdobramento de spin, é pouco sensível ao comprimento característico e depende fortemente do valor de $x_{p}$. Por isso fixei o valor de $\ell$ e usei $x_{p}$ como parâmetro ajustável. $\mathrm{O}$ procedimento contrário também foi tentado, ou seja, fixar $x_{p}=0.065$, que é o valor nominal, e variar $\ell$. Entretanto, com esta opção eu não consegui obter resultados compatíveis com os dados experimentais.
} 

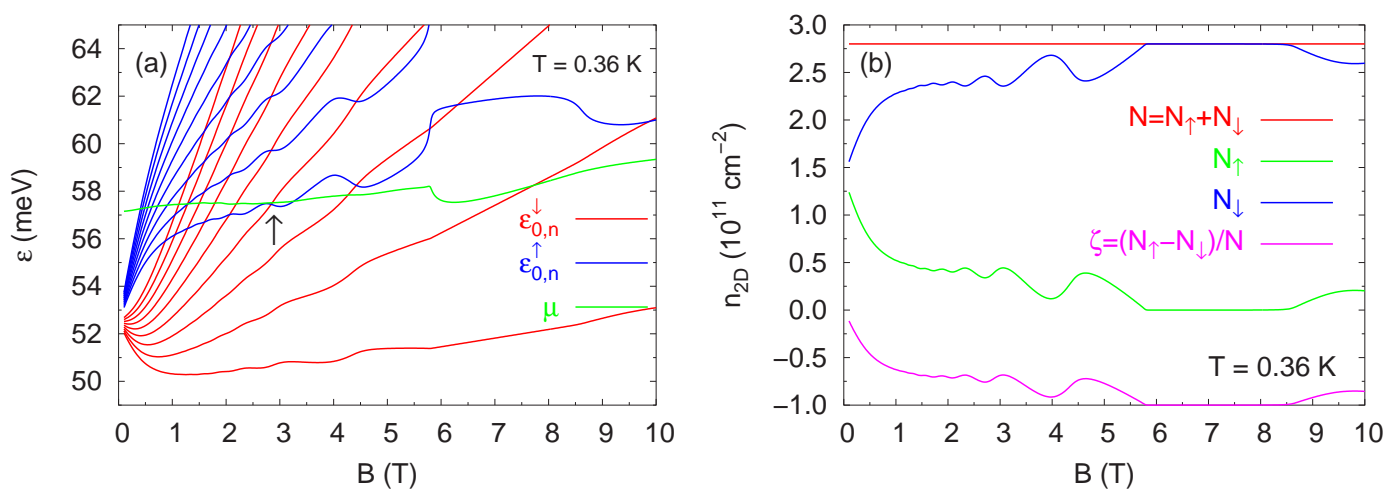

Figura 5.7: Diagrama dos níveis de Landau (a) mostrando o desdobramento de spin gigante oriundo da interação de troca $s-d$. Note que a densidade total (b) é constante e o potencial químico oscila em função do campo magnético. Em $B \sim 6 \mathrm{~T}$ o nível $\varepsilon_{1,0}^{\uparrow}$ varia abruptamente devido a dependência da interação de troca e correlação (XC) com o polarização do 2DEG: quando $\zeta \rightarrow-1$, a contribuição de XC para o desdobramento de spin aumenta drasticamente. Note o cruzamento de níveis em $B \sim 3 \mathrm{~T}$. Este cruzamento, e a proximidade com o potencial químico $\mu$, é o responsável pelo aparecimento de anomalias nos gráficos de magnetotransporte.

Note que é a energia da sub-banda que incorpora o efeito da interação de troca s$d$, responsável pelo desdobramento de spin gigante, ${ }^{11}$ bem como as contribuições do confinamento estrutural e da interação de Coulomb. A Figura 5.7(a) mostra o diagrama de níveis de Landau para $T=0.36 \mathrm{~K}$. Para campos até $B \sim 2 \mathrm{~T}$ a dependência com o campo magnético tem uma forte influência da função de Brillouin da interação de troca s-d. Quando esta satura, a energia de cada nível de Landau passa a ter o comportamento linear com $B$ proveniente da energia de cíclotron.

Entretanto, diferentemente do modelo "rígido" para os LLs da Eq. (5.1) usado pelo grupo experimental, aqui o potencial, e consequentemente a estrutura eletrônica, varia autoconsistentemente à medida que algum parâmetro externo é alterado. Assim, as formas das curvas dos LLs não é simplesmente uma superposição de contribuições do tipo função de Brillouin (troca s-d) mais uma função linear (cíclotron + Zeeman ordinário), como pressuposto na análise do grupo experimental. Este comportamento, devido a autoconsistência, é bem exemplificado na região de campo magnético em que o 2DEG fica polarizado. A contribuição de troca-correlação é bastante sensível à polarização do 2DEG, sendo o efeito mais acentuado quando $\zeta \rightarrow-1$ (cf. Fig. 4.3 na pág. 84). O resultado é um aumento abrupto do desdobramento de spin, resultando numa "expulsão" do nível $|0, \uparrow\rangle$ em

\footnotetext{
${ }^{11} \mathrm{~A}$ contribuição da interação de troca s-d é incorporada via o respectivo potencial dependente de spin, o que acarreta automaticamente um desdobramento de spin gigante nos níveis de energia calculados. Já a análise do grupo experimental não calcula este desdobramento, mas utiliza em seu modelo o desdobramento de spin medido experimentalmente.
} 
$B \sim 6$ T. Esta mesma autoconsistência faz com que o nível $|0, \uparrow\rangle$ seja "capturado" em $B \sim 8 \mathrm{~T}^{12}$

Como a densidade do 2DEG é constante, o potencial químico varia com o campo $B$. Devido ao efeito Zeeman gigante, já em $B \sim 3$ T o último nível de spin para cima cruza o potencial químico e o sistema fica quase que completamente polarizado. Isto pode ser acompanhado na Fig. 5.7(b), que mostra as densidades bidimensionais de spin para cima e spin para baixo. A densidade $n_{2 D}$ total é calculada usando a Eq. (4.61),

$$
n_{2 D}=\int_{-\infty}^{\infty} g(\varepsilon) f(\varepsilon) \mathrm{d} \varepsilon
$$

na qual $g(\varepsilon)$ é a densidade de estados (4.54) e $f(\varepsilon)$ é a função de Fermi (4.59). Para as densidades parciais, basta apenas considerar um spin na densidade de estados da Eq. (4.61).

Magnetotransporte: IQHE e SdH Uma vez calculada a estrutura eletrônica eu utilizo modelos apropriados para calcular outras grandezas experimentalmente relevantes. Aqui eu utilizo o modelo descrito no Cap. 4 para a magnetocondutividade de um 2DEG, Eq. (4.74),

$$
\begin{gathered}
\sigma_{x x}=\frac{e^{2}}{h} \frac{2}{\pi} \int_{-\infty}^{\infty}\left(-\frac{\partial f(\varepsilon)}{\partial \varepsilon}\right) \sum_{i, n, \sigma_{z}}\left(n+\frac{1}{2}\right) \exp \left[-\left(\frac{\varepsilon-\varepsilon_{i, n}^{\sigma_{z}}}{\Gamma_{\mathrm{ext}}^{\sigma_{z}}}\right)^{2}\right] \mathrm{d} \varepsilon, \\
\sigma_{x y}=\frac{e n_{2 D}\left[g_{\text {ext }}\right]}{B}+\frac{e^{2}}{h} \frac{2}{\pi} \int_{-\infty}^{\infty}\left(-\frac{\partial f(\varepsilon)}{\partial \varepsilon}\right) \sum_{i, n, \sigma_{z}}\left(n+\frac{1}{2}\right) \frac{\Gamma_{\mathrm{ext}}^{\sigma_{z}}}{\hbar \omega_{c}} \exp \left[-\left(\frac{\varepsilon-\varepsilon_{i, n}^{\sigma_{z}}}{2 \Gamma_{\mathrm{ext}}^{\sigma_{z}}}\right)^{2}\right] \mathrm{d} \varepsilon,
\end{gathered}
$$

para então calcular a magnetorresistividade, Eq. (4.76),

$$
\rho_{x x}=\frac{\sigma_{x x}}{\sigma_{x x}^{2}+\sigma_{x y}^{2}} \quad, \quad \rho_{x y}=\frac{\sigma_{x y}}{\sigma_{x x}^{2}+\sigma_{x y}^{2}},
$$

que é a grandeza experimentalmente acessível. Os resultados obtidos estão na Fig. 5.8.

As oscilações de Shubnikov-de Haas na Fig. 5.8(a) reproduzem tanto o aparecimento do pico anômalo em $B \simeq 3.2 \mathrm{~T}$ quanto o deslocamento das curvas para baixos campos magnéticos à medida que a temperatura aumenta. O pico

\footnotetext{
${ }^{12}$ Note que a Fig. 5.7(a) mostra o centro de cada nível de Landau. Lembre, porém, que há estados com energias mais altas e baixas devido ao alargamento $\Gamma$. Por isso, como no caso do nível $|0, \uparrow\rangle$ em $B \sim 8 \mathrm{~T}$, apesar do centro do LL estar acima do potencial químico, este nível ainda está parcialmente ocupado.
} 


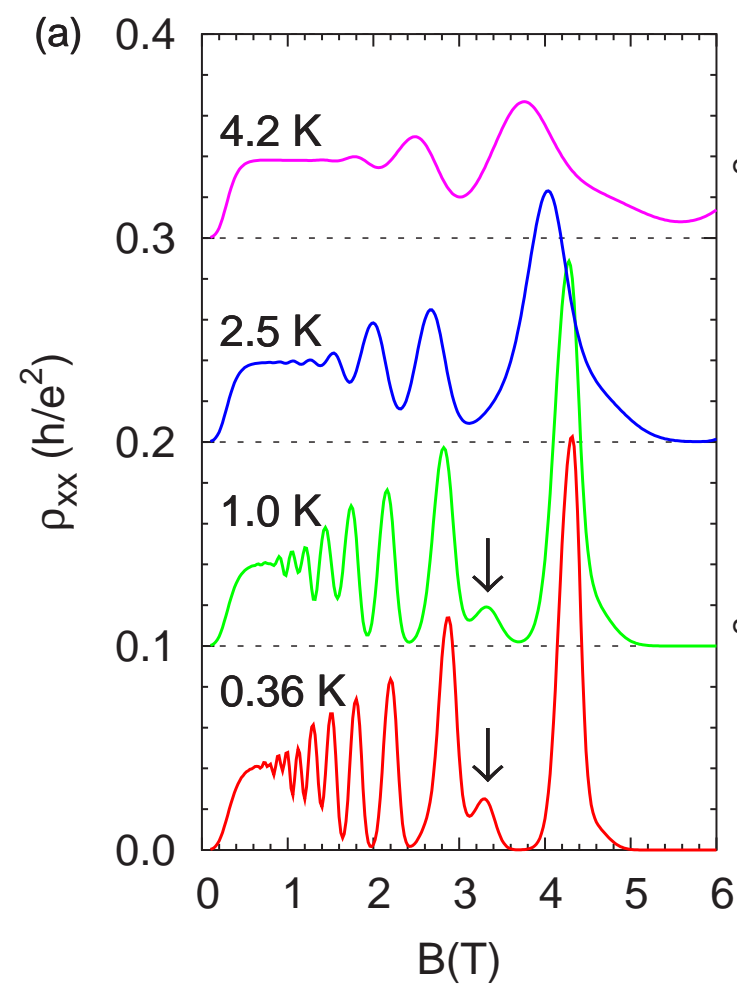

(b)
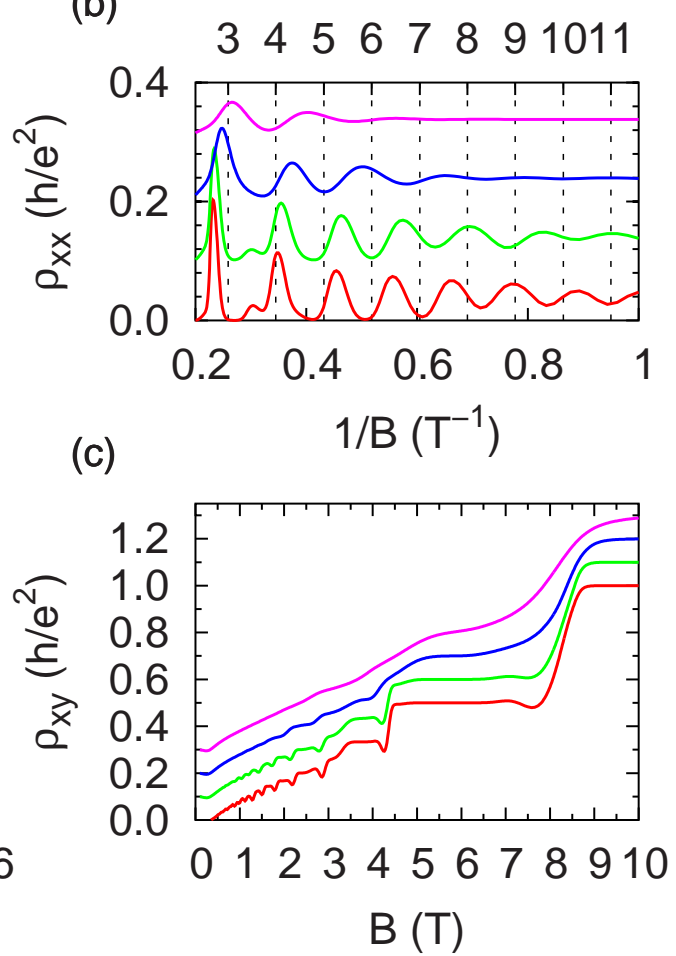

Figura 5.8: Resultados para o magnetotransporte para diversas temperaturas. As oscilações de Shubnikov-de Haas (a) são bastante semelhantes aos resultados experimentais da Fig. 5.2(a). Note o aparecimento do pico anômalo em $B \simeq 3.2 \mathrm{~T}$ e o deslocamento dos demais picos para baixos campos à medida que a temperatura aumenta. O painel (b), com os dados de (a) em função de $1 / B$, mostra a aperiodicidade das oscilações, semelhante à Fig. 5.4 experimental. Os fatores de preenchimento $\nu$ foram calculados usando a densidade $n_{2 D}=2.8 \times 10^{11} \mathrm{~cm}^{-2}$. A magnetorresistência Hall está apresentada em (c). Em (a) o comportamento a baixo campo $(B<0.5 \mathrm{~T})$ é devido à soma truncada de níveis de Landau no procedimento numérico. Para $B \rightarrow 0$ seria necessário considerar um número infinito de níveis de Landau.

anômalo ocorre justamente na região em que os níveis de Landau $|3, \downarrow\rangle$ e $|0, \uparrow\rangle$, e o potencial químico $\mu$ cruzam: mais precisamente, o pico anômalo surge quando o nível $|0, \uparrow\rangle$ cruza $\mu$ uma segunda vez em $B \simeq 3.2 \mathrm{~T}$, como ilustrado na Fig. 5.7(a).

Note que, diferindo do resultado experimental, para $T=1.0 \mathrm{~K} \mathrm{o}$ pico anômalo ainda é bem destacado enquanto na Fig. 5.2 ele está praticamente sobreposto ao pico de SdH usual. A explicação para isto pode estar relacionada com o fato da posição do pico anômalo ser bastante sensível a pequenas variações dos diversos parâmetros que eu ajusto no modelo, como $x_{p}, T_{0}$, $\Gamma$ e $\Gamma_{e x t}^{\sigma_{z}}$. Assim, pode ser que um ajuste mais fino destes parâmetros corrija a posição deste pico.

Outra possível explicação é de caracter mais fundamental, e envolve a acurácia da descrição da dependência do sistema com a temperatura. No modelo que utilizo, a temperatura entra via a função de Brillouin $B_{S}$ e via a função de 
Fermi $f$. Enquanto o efeito desta última é acentuar as oscilações à medida que $T$ aumenta, a função de Brillouin afeta decisivamente a posição dos picos uma vez que ela determina a posição dos LLs. É justamente a dependência de $B_{S}$ com a temperatura que resulta no deslocamento dos demais picos de $\mathrm{SdH}$ para campos mais baixos quando $T$ aumenta. Aqui eu utilizo uma temperatura efetiva $T_{0}$ constante. Entretanto, Knobel [145] precisou utilizar valores diferentes de $T_{0}$ para cada valor de $T$, para ajustar curvas de desdobramento de spin usando a função de Brillouin, indicando que a dependência correta com a temperatura pode ser mais intrincada.

Quanto ao deslocamento dos demais picos de SdH com a temperatura, percebe-se que os resultados que eu apresento na Fig. 5.8 estão mais próximos dos dados experimentais, Fig. 5.2(a), que a simulação da Ref. [37], Fig. 5.2(b). Por exemplo, acompanhe o pico em $B \sim 4 \mathrm{~T}$ à medida que $T$ aumenta. Para $T=0.36 \mathrm{~K}$ e $1.0 \mathrm{~K}$ eles mudam pouco de posição. Já ao passar para $T=2.5 \mathrm{~K}$ o deslocamento é bem mais acentuado. Este comportamento é muito próximo aos resultados experimentais e não é reproduzido no modelo da Ref. [37], Fig. 5.2(b). Acredito que foi o caracter autoconsistente do cálculo da estrutura eletrônica que possibilitou este acerto.

A aperiodicidade observada nas oscilações quânticas, Fig. 5.4, também é reproduzida na Fig. 5.8(b). Note que há aproximadamente um mínimo de SdH para cada fator de preenchimento para $\nu \leqslant 7$, indicando uma separação dos LLs; já para $\nu>7$ há 3 mínimos para 4 valores de $\nu$. Este resultados são similares aos dados experimentais. Entretanto, para obter este resultado eu precisei utilizar uma temperatura efetiva $T_{0}=0.55 \mathrm{~K}$, menor que o valor de $1.55 \mathrm{~K}$ usado na simulação do grupo experimental. ${ }^{13}$ Simulações usando $T_{0}=1.55 \mathrm{~K}$ resultaram em oscilações de $\mathrm{SdH}$ para baixos campos que englobavam 2 LLs. Já para $B>2 \mathrm{~T}$, a periodicidade das oscilações concordam com o experimento para os dois valores de $T_{0}$.

O painel (c) da Fig. 5.8 mostra os resultados para a resistividade Hall. Uma característica que chama atenção são as "depressões" de $\rho_{x y}$ no final de cada platô. Elas ocorrem sistematicamente nas mesmas regiões em que $\rho_{x x}$ é máximo, quando $\mu$ passa pelos estados estendidos, mas não são observadas nos dados experimentais a altos campos. Estas depressões são anomalias do modelo que usa uma gaussiana para descrever os estados estendidos no centro dos níveis de Landau. Para o surgimento dos platôs no efeito Hall quântico inteiro, é necessário que $\mu$ esteja no gap de mobilidade, o que não ocorre perfeitamente se os estados

\footnotetext{
${ }^{13} \mathrm{~A}$ temperatura efetiva $T_{0}$ "regula" a subida da função de Brillouin para o valor de saturação. Quanto maior (menor) $T_{0}$ menos (mais) acentuada é a saturação e, consequentemente, menor (maior) é a separação de spin para baixos campos.
} 

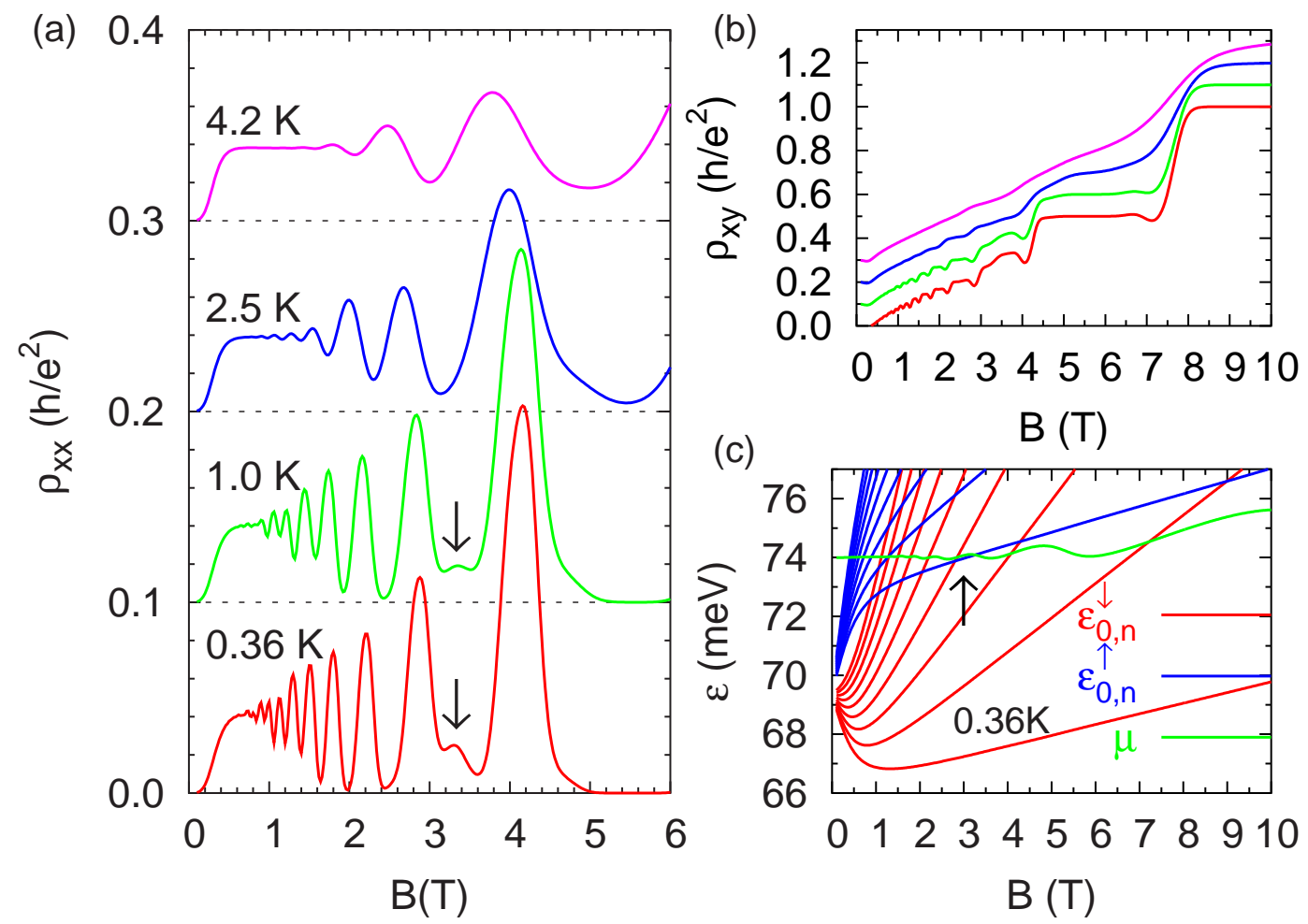

Figura 5.9: Resultados do cálculo na aproximação Hartree para $\rho_{x x}$ (a), $\rho_{x y}$ (b) e para os níveis de Landau (c). As oscilações de Shubnikov-de Haas deslocam-se para baixos campos à medida que $T$ aumenta. O pico anômalo ocorre próximo de $3.2 \mathrm{~T}$ (a) e é resultado de cruzamento de níveis de Landau próximo do potencial químico (curva verde).

estendidos têm uma distribuição gaussiana. ${ }^{14}$ Esta anomalia também se reflete a amplitude dos picos de $\mathrm{SdH}$, mas sem alterar as demais características. Excluindo a questão desta anomalia em $\rho_{x y}$, as curvas de resistividade apresentam os platôs que indicam o regime de efeito Hall quântico inteiro.

Já em $B \sim 3.0 \mathrm{~T}$, a curva de $\rho_{x y}$ não tem a oscilação observada nos dados experimentais [indicada na Fig. 5.1(a)]. Por um lado, pode ser que a anomalia que mencionei está obscurecendo este efeito. Por outro lado, como mencionado na discussão dos resultados experimentais (nota 4 na pág. 112), aquela oscilação parece refletir não só o cruzamento de níveis nesta região de campo, mas também um início de mudança de platô. Entretanto, nas minhas simulações eu não consegui encontrar um conjunto de parâmetros tal que a resistividade Hall ultrapassasse o valor $\rho_{x y}=0.25 h / e^{2}$ e depois diminuísse novamente, como na figura experimental 5.1 (a) em $B \simeq 2.9 \mathrm{~T}$.

\footnotetext{
${ }^{14}$ Testes usando densidades de estado parabólicas, como no modelo original de Ando e Uemura [118], eliminaram esta anomalia. Entretanto, o conjunto dos resultados para magnetotransporte ficou pior que o modelo usando alargamentos gaussianos.
} 
Simulação Hartree Os resultados apresentados até agora incluem a contribuição de troca-correlação do gás de elétrons na aproximação da densidade de spin local. Para ressaltar o papel desta contribuição, aqui eu apresento as mesmas simulações, mas na aproximação Hartree, ou seja, $v_{x c}^{\sigma_{z}}=0$ no potencial efetivo. Os resultados estão apresentados na Fig. 5.9. Para reproduzir os resultados experimentais nesta aproximação, eu precisei alterar apenas um parâmetro: a concentração planar de Mn usada nas simulações foi $x_{p}=0.085$. Isto ocorre pois a interação de troca-correlação contribui para o desdobramento de spin. Assim, sem esta contribuição, preciso de uma concentração maior de Mn para manter um desdobramento de spin adequado que ajuste a posição dos LLs de forma a reproduzir os resultados de magnetotransporte. Entretanto, este valor é maior que o valor nominal $x_{p}=0.065$ do poço quântico. ${ }^{15}$ De fato, este valor está relativamente próximo à estimativa que fiz na Seção 5.2, pág. 119, baseada na concentração efetiva $x_{e f f}$, que resultou em $x_{p}=0.096$. Assim, concluo que, tanto na minha simulação Hartree, quanto na simulação do grupo experimental, ambas precisam de uma quantidade maior de Mn para compensar a não inclusão do desdobramento de spin devido a efeitos de muitos corpos (troca e correlação).

Por outro lado, o valor $x_{p}=0.035$ usado nas simulações com XC é bem menor que o nominal. Isto pode ter duas explicações, não excludentes: (i) de fato a quantidade de Mn realmente incorporada nas amostras foi menor que o valor que se almejava, e/ou (ii) o potencial de troca-correlação utilizado está resultando um desdobramento de spin exagerado. De qualquer maneira, os resultados utilizando XC estão mais consistentes com os dados experimentais, principalmente no que se refere ao valor da concentração $x_{p}$ de Mn. No próximo capítulo eu apresento um sistema em que a inclusão da contribuição de trocacorrelação é essencial para reproduzir os efeitos de ferromagnetismo itinerante, transição de fase e ciclo de histerese observados experimentalmente [21], o que não é possível quando utilizo apenas a contribuição Hartree para a interação de Coulomb.

Energia total Usando a prescrição da teoria do funcional da densidade (Sec. 4.2), eu calculo a energia total do sistema,

$$
\begin{aligned}
E\left[n_{\uparrow}, n_{\downarrow}\right]=\int \varepsilon g(\varepsilon) & f(\varepsilon) \mathrm{d} \varepsilon-\frac{1}{2} \int v_{h}(z) n(z) \mathrm{d} z \\
& -\sum_{\sigma_{z}} \int v_{x c}^{\sigma_{z}}(z) n_{\sigma_{z}}(z) \mathrm{d} z+E_{x c}\left[n_{\uparrow}, n_{\downarrow}\right]-T S_{s}\left[n_{\uparrow}, n_{\downarrow}\right] .
\end{aligned}
$$

\footnotetext{
${ }^{15} \mathrm{O}$ dito valor nominal é o valor que presume-se incorporado na amostra durante o crescimento por MBE. Contudo, não foram realizadas outras medidas para se confirmar o conteúdo que de fato foi incorporado no DMQW [R. Knobel, comunicação privada].
} 

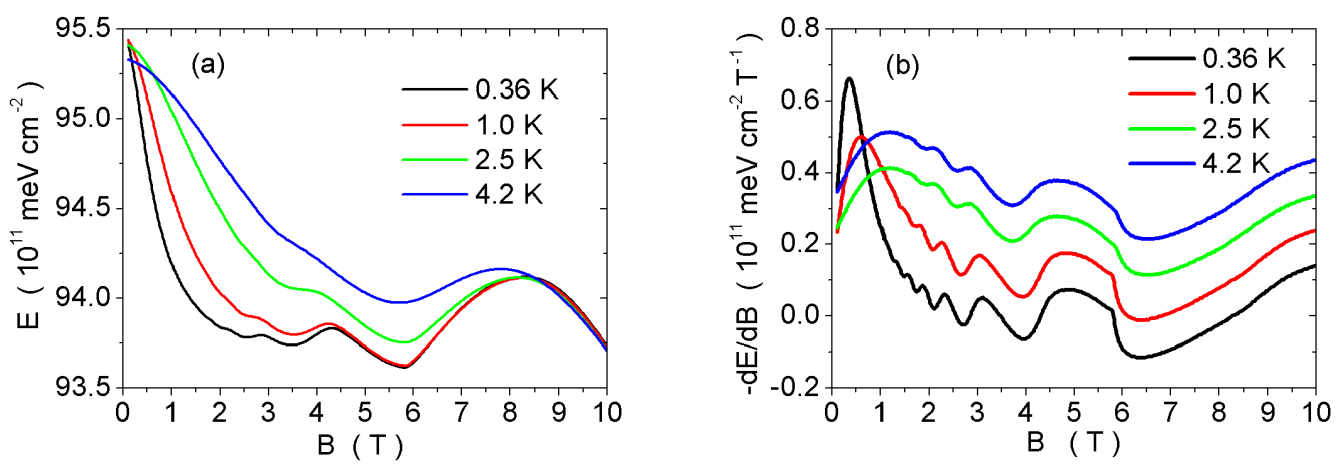

Figura 5.10: Energia total do 2DEG em função do campo magnético. A dependência da energia total (a) com o campo magnético tem duas contribuições marcantes. A primeira é a interação de troca $s-d$, que diminui a energia e segue um comportamento do tipo função de Brillouin. A segunda vem da contribuição orbital dos níveis de Landau, que confere o caracter oscilatório da energia em função de $B$. O painel (b) contem a primeira derivada com relação a $B$ das curvas de energia do painel (a).

Como comentado na Sec. 4.2, esta é a energia do sistema de muitos corpos interagente. A Fig. 5.10(a) mostra a energia total (5.9) em função do campo magnético para diferentes temperaturas. Este gráfico mostra bem o efeito com o campo magnético das duas principais contribuições para a energia total:

1. A contribuição da interação de troca $s-d$ para $E\left[n_{\uparrow}, n_{\downarrow}\right]$ domina em baixos campos. O desdobramento de spin gigante faz com que o 2DEG fique polarizado, ou seja, com mais elétrons com spin $|\downarrow\rangle$ que com spin $|\uparrow\rangle$. Como, para baixos campos, a energia dos níveis $\varepsilon_{1, n}^{\downarrow}$ diminui com $B$ [cf. Fig. 5.7(a)], o resultado é que a energia total também diminui em baixos campos.

2. A contribuição da energia orbital (cíclotron), passa a dominar quando a função de Brillouin satura ou quando a energia de cíclotron $\hbar \omega_{c}$ é grande o suficiente comparada com outros termos. A contribuição orbital oscila à medida que o nível de Fermi percorre os vários níveis de Landau, similar ao exemplo exemplo da Fig. 4.6 na pág. 98.

Na Figura 5.10(b) eu apresento, apenas a título ilustrativo, a primeira derivada com relação ao campo magnético das curvas de energia total, $-\mathrm{d} E / \mathrm{d} B$. Note, porém, que as curvas resultantes não correspondem à magnetização do 2DEG. Isto porque, como apontado na Sec. 4.3.5, a magnetização orbital é obtida efetuando a derivada parcial da energia orbital com relação ao campo magnético, Eq. (4.64), mantendo as demais variáveis constantes $\left(T, n_{\sigma_{z}}\right)$. Entretanto, a energia $E\left[n_{\uparrow}, n_{\downarrow}\right]$, Fig. $5.10($ a), contém várias contribuições que variam com o campo magnético. Além das contribuições de troca $s$-d, orbital, e 
Zeeman, que têm uma dependência direta com o campo magnético, as contribuições de confinamento estrutural, de Hartree, de troca-correlação e de energia cinética também variam com $B$ via as densidades parciais $n_{\uparrow}$ e $n_{\downarrow}$. Desta forma, as curvas de $-\mathrm{d} E / \mathrm{d} B$ da Fig. 5.10(b) se assemelham àquelas da magnetização obtidas experimentalmente apenas porque as oscilações da energia total têm uma forte contribuição da energia orbital para $B>2 \mathrm{~T}$.

No caso do exemplo da Fig. 4.6, pág. 98, como o sistema era nãomagnético e não-interagente, a única contribuição para a energia que variava com $B$ era a própria energia orbital, e as densidades $n_{\sigma_{z}}$ permaneciam constantes. Assim, naquele caso específico, a derivada total da energia com relação a $B$ era igual a derivada parcial. Aqui, para simular as oscilações da magnetização orbital (efeito de Haas-van Alphen), eu teria que efetuar a derivada parcial da energia orbital, $-\partial E_{\text {orb }} / \partial B$, apropriadamente [126]. Este cálculo não foi efetuado no trabalho desta tese, mas será um dos tópicos a serem abordados em trabalhos futuros.

\subsection{Discussões}

Neste capítulo eu investiguei teoricamente os resultados experimentais das Refs. [37, 38] para o magnetotransporte de 2DEGs confinados em poços quânticos digitais magnéticos do grupo II-VI. Utilizando a formulação teórica baseada na DFT/LSDA para o cálculo da estrutura eletrônica (Sec. 4.2), o modelo para o potencial dependente de spin em DMHs (Sec. 1.4), e o modelo para o magnetotransporte apresentado na Sec. 4.4, eu fui capaz de reproduzir os principais resultados experimentais das referências mencionadas. São eles: o aparecimento de um pico de resistividade anômalo em $B \simeq 3.2 \mathrm{~T}$ devido ao cruzamento de níveis de Landau próximo ao potencial químico, e o deslocamento das curvas de $\rho_{x x}$ para campos mais baixos à medida que a temperatura aumenta, efeito este resultado da dependência da intensidade da interação de troca $s$-d (função de Brillouin) com a temperatura.

Alguns pontos relativos a estes resultados merecem destaque. Primeiramente, eu mostrei que a contribuição de muitos corpos (troca-correlação) é considerável neste sistema, sendo responsável por parte relevante do desdobramento de spin dos níveis eletrônicos. Ao considerar esta contribuição, eu fui capaz de reproduzir os resultados experimentais usando uma concentração de Mn, menor, porém mais compatível com a concentração nominal de Mn na amostra. Ao contrário, na análise do grupo experimental, todo o desdobramento de spin era devido a interação de troca s-d. Portanto, era necessário utilizar uma concentração de Mn maior que a de fato existente na amostra para atingir o desdobramento 
de spin necessário, que reproduz o cruzamento de LLs nos valores de $B$ corretos. Este ponto é confirmado pela minha simulação na aproximação Hartree, na qual precisei usar uma concentração de Mn maior que a nominal para reproduzir os dados experimentais, uma vez que não há XC contribuindo para o desdobramento de spin.

Em segundo lugar, o caracter autoconsistente do cálculo que eu efetuei permitiu descrever melhor a dependência da estrutura eletrônica do sistema com o campo magnético, refletindo nos resultados para magnetotransporte. De uma forma geral, os perfis das curvas de Shubnikov-de Haas ficaram bem próximos às curvas experimentais.

Um terceiro ponto a ser ressaltado é que os resultados apresentados neste capítulo servem como uma confirmação adicional da adequação dos modelos para o potencial dependente de spin apresentados nos capítulos anteriores. Aqui eu calculei o efeito da interação de troca $s-d$ dos elétrons com as impurezas magnéticas via um potencial efetivo que entra na equação de Schrödinger. Para tanto eu utilizei o modelo de concentração efetiva considerando aglomeração antiferromagnética de singletos e tripletos, e diluição devido a segregação. 


\section{Capítulo 6}

\section{Ferromagnetismo Itinerante no Regime de Efeito Hall Quântico}

\section{Conteúdo}

Vários experimentos recentes de magnetotransporte no regime de efeito Hall quântico apresentam evidências da formação de estados de ferromagnetismo itinerante, efeito este chamado de ferromagnetismo de efeito Hall (QHF). A literatura corrente sugere a formação de domínios ferromagnéticos, e de barreiras entre estes domínios, para explicar estes resultados. Aqui eu analiso os resultados experimentais de Jaroszyński et al. [21] para o magnetotransporte em um poço quântico digital magnético de (Cd,Mn)Te, e proponho uma explicação alternativa para estes resultados. Os autores usaram o efeito Zeeman gigante presente em DMHs para "induzir externamente" o cruzamento de níveis de Landau, em campos moderados, do 2DEG confinado, reproduzindo assim as condições necessárias para a ocorrência de QHF. Eu calculo a estrutura eletrônica deste sistema usando o formalismo de Kohn-Sham da DFT/LSDA na aproximação da massa efetiva. Em seguida eu calculo o magnetotransporte, reproduzindo satisfatoriamente os resultados experimentais da Ref. [21]. O cálculo apresenta ciclos de histerese e uma temperatura crítica, evidências da ocorrência de QHF, mas sem precisar recorrer da hipótese de formação de domínios.

6.1 Introdução . . . . . . . . . . . . . . . . . . . . 135

6.2 Resumo do resultados experimentais . . . . . . . . . . 136

6.3 Resultados teóricos . . . . . . . . . . . . . . . . . . . . 145

6.4 Discussões . . . . . . . . . . . . . . . . . . . . . 159 


\subsection{Introdução}

O sistema investigado no capítulo anterior é singular no sentido que atende uma série de condições para possibilitar o aparecimento do pico anômalo de resistividade longitudinal. O desdobramento de spin gigante devido a interação de troca s-d fez que níveis de Landau com spins opostos cruzassem justamente próximo do potencial químico. O resultado foi o aparecimento de um pico anômalo no gráfico de $\rho_{x x}$. Naquele capítulo, apliquei a formulação teórica apresentada no Cap. 4 para estudar resultados experimentais de magnetotransporte em função da temperatura $[37,38]$. O modelo reproduziu bem o deslocamento dos picos de SdH com $T$ e o aparecimento do pico anômalo. Naquele sistema, a inclusão da contribuição de muitos corpos de troca-correlação foi importante devido a sua contribuição para o desdobramento de spin dos níveis eletrônicos.

Agora a intenção é ir um passo adiante. Devido a interação de troca $s$-d, o desdobramento de spin gigante permite que níveis com spins opostos cruzem próximo do potencial químico. Nestas condições, existe a possibilidade de ocorrer uma quebra de simetria de spin com a formação de um estado ferromagnético. Ou seja, ao invés dos dois estados ficaram sobrepostos (degenerados), é mais favorável que todos os elétrons fiquem apenas com uma orientação de spin. Este corresponde a um estado ferromagnético dos elétrons itinerantes, fenômeno chamado de ferromagnetismo de efeito Hall quântico (QHF - quantum Hall ferromagnetism) [20]. Enquanto os trabalhos das Refs. [37, 38] não apresentaram evidências deste efeito, nos últimos anos outros trabalhos sob condições similares resultaram em várias evidências concretas de ferromagnetismo itinerante [21-35]. Em particular, o trabalho de Jaroszyński et al. [21] registrou um pico de resistividade anômalo que sofre histerese magnética. Este resultado foi interpretado como consequência de uma transição de fase para um regime de QHF.

Há poucos trabalhos teóricos na literatura abordando estes resultados experimentais recentes. Os principais são cálculos Hartree-Fock para esta classe de sistemas [153-157], e mostram que a interação de troca seria capaz de estabilizar o estado ferromagnético. Tanto nestes trabalhos teóricos quanto nos experimentais, há um aparente "consenso" que o aparecimento do QHF está relacionado com a existência de domínios na distribuição espacial dos elétrons que formam o 2DEG. No regime de QHF, na região correspondente aos platôs de $\rho_{x y}$, a resistividade longitudinal $\rho_{x x}$ é praticamente nula. Porém, nestes sistemas ocorreria condução nas barreiras entre domínios, resultando nos picos de resistividade anômalos observados. Similar aos ferromagnetos comuns, estes domínios seriam responsáveis pela histerese que é observada nos picos anômalos.

Este capítulo da tese analisa justamente o trabalho de Jaroszyński 
et al. [21] em DMHs de (Cd,Mg)Te/(Cd,Mn)Te. Eu mostro que um cálculo padrão de estrutura eletrônica nas aproximações de massa efetiva e função envelope, no contexto da teoria do funcional da densidade dependente de spin (Cap. 4), fornece resultados com o pico anômalo e histerese, reproduzindo os resultados da Ref. [21].

Uma vez que o modelo para a estrutura eletrônica e para o magnetotransporte aqui adotado não inclui a hipótese de formação de domínios (apesar de também não excluir a possibilidade de existência destes), os resultados apresentados neste capítulo são uma explicação alternativa para os efeitos observados experimentalmente. Os trabalhos teóricos (Hartree-Fock) que usam a hipótese de formação de domínios [154, 157] mostram que, quando há coincidência de níveis de Landau de spins opostos, (i) o estado fundamental é de QHF, e (ii) as excitações de mais baixa energia correspondem à formação de barreiras entre domínios. Entretanto, não há nenhum cálculo mostrando o magnetotransporte devido a estas barreiras.

Na próxima seção, eu apresento os resultados experimentais de Jaroszyński et al. [21] e a abordagem teórica usada pelos autores para entender estes resultados. Na seção seguinte, eu utilizo o modelo para DMHs dopadas usada nesta tese para simular o experimento da Ref. [21]. Para finalizar, eu retomo a análise do experimento de Knobel et al. [37] e Harris et al. [38] (Cap. 5) para entender porque os autores não observaram sinal de ferromagnetismo itinerante em seus experimentos.

\subsection{Resumo dos resultados experimentais}

Descrição do sistema e medidas O sistema investigado por Jaroszyński et al. [21] consiste de um poço quântico digital magnético (DMQW) n-dopado por modulação, semelhante ao da Fig. 6.1. O DMQW, crescido na direção (100), tem largura $100 \AA$ e possui três barreiras digitais (monocamadas) de MnTe. Experimentos de fotoluminescência indicam que a concentração "média" de Mn é $0.020 \pm 0.005 .^{1}$

As barreiras laterais são de $\mathrm{Cd}_{0.8} \mathrm{Mg}_{0.2} \mathrm{Te}$. A concentração de $\mathrm{Mg}$ é tal que a diferença dos gaps nas interfaces do poço é de $330.8 \mathrm{meV}$ (cf. Tabela 6.2,

\footnotetext{
${ }^{1}$ Jaroszyński et al. [21] não são claros sobre como eles estimaram a concentração de Mn. Em semicondutores magnéticos diluídos bulk, a concentração de Mn pode ser estimada ajustando as curvas de magnetofotoluminescência usando a função de Brillouin. Já nas heteroestruturas digitais, o procedimento não é tão direto: é preciso levar em consideração a sobreposição da função de onda eletrônica com os momentos de Mn. Portanto, acredito que o valor indicado por Jaroszyński et al. [21] como sendo a concentração média é, na realidade, o que eu defini como concentração efetiva $x_{e f f}$, Eq. (2.32) da pág. 40. De fato, o próprio modelo teórico usado pelo autores para simular o desdobramento de spin usa uma concentração de 0.0112 , cf. Eq. (6.1). Para obter os resultados que apresento na próxima seção eu uso concentração nominal $x_{p}=0.115$, que corresponde a uma concentração efetiva $x_{e f f}=0.009$.
} 


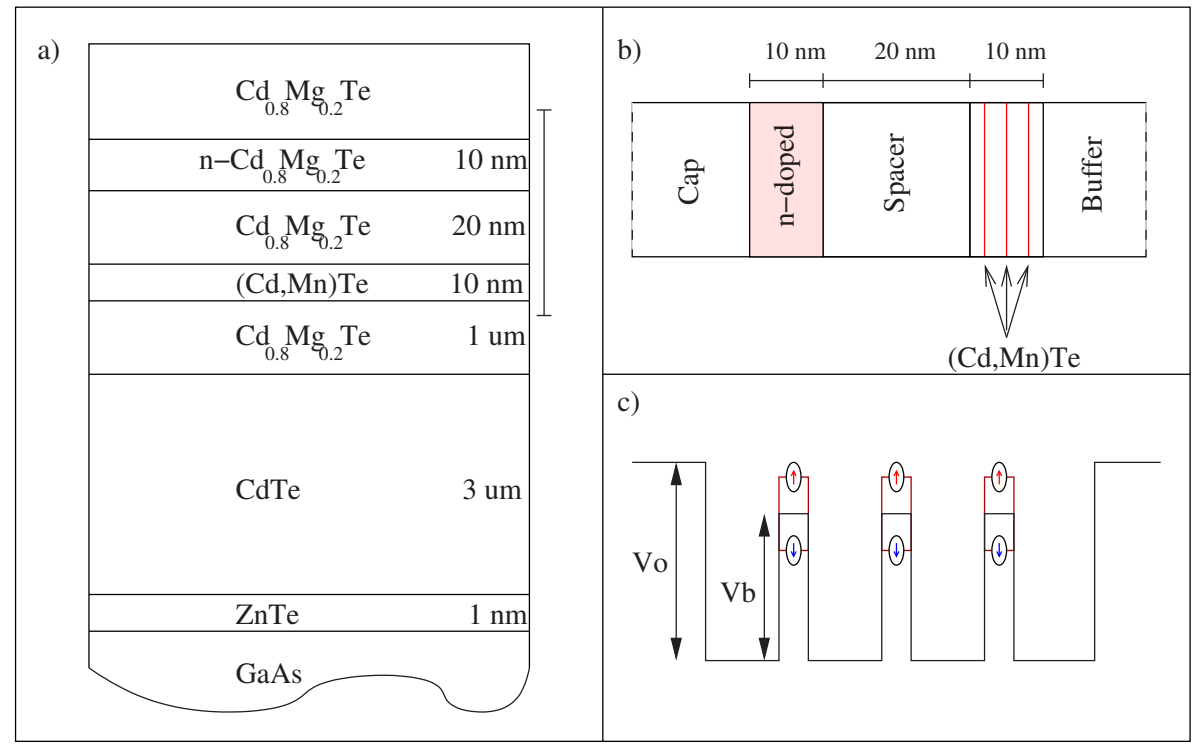

Figura 6.1: Heteroestrutura Digital Magnética usada por Jaroszyński et al. [21] para realizar medidas de magnetotransporte. A região de interesse é o poço quântico restrito à região indicada em (a). O painel (b) é uma ampliação desta região, mostrando o poço com as camadas magnéticas e a região dopada. O painel (c) representa o perfil de potencial da banda de condução. As camada magnéticas são responsáveis por uma modulação do potencial dependente de spin.

pág. 145). Atribuindo $75 \%$ para a banda de condução [49] a altura do poço quântico fica $v_{0}=248.1 \mathrm{meV}$.

Iodo é usado como dopante do tipo $n$, substituindo o Telúrio ${ }^{2}$ numa região distante $200 \AA$ do poço. O conjunto poço-barreira é crescido sobre uma camada composta por um substrato de GaAs, seguido de uma camada de ZnTe de 10 Å e de um buffer de CdTe de $3 \mu \mathrm{m}$ [Fig. 6.1(a)]. Além da dopagem, um terminal metálico soldado sobre a camada superior da heteroestrutura torna possível variar a densidade do 2DEG confinado no poço, obtendo valores para $n_{s}$ entre (1.6 e $3.6) \times 10^{11} \mathrm{~cm}^{-2}$, determinados com medidas de efeito Hall a baixo campo nas temperaturas de $10 \mathrm{~K}$ e $23 \mathrm{~K}$.

As medidas de magnetotransporte foram efetuadas numa barra Hall de $0.5 \mathrm{~mm} \times 1 \mathrm{~mm}$ cujos terminais foram soldados com Índio. A mobilidade eletrônica na amostra é $1.6 \times 10^{4} \mathrm{~cm}^{2} / \mathrm{Vs}$, possivelmente limitada por espalhamentos devido à desordem de curto alcance das impurezas magnéticas e das imperfeições ligas da heteroestrutura [21]. Os experimentos foram realizados em campos magnéticos de até $18 \mathrm{~T}$ e temperaturas tão baixas quanto $0.03 \mathrm{~K}$. A amostra também foi rotacionada de forma a variar o ângulo $\theta$ entre a interface normal e o campo magnético $\boldsymbol{B} .^{3}$

\footnotetext{
${ }^{2}$ Quando o Iodo, cuja configuração eletrônica é $[\mathrm{Kr}] 5 s^{2} 4 d^{10} 5 p^{5}$, substitui o Telúrio ( $\left.[\mathrm{Kr}] 5 s^{2} 4 d^{10} 5 p^{4}\right)$, o elétron adicional da camada $p$ faz com que a dopagem seja do tipo $n$.

${ }^{3} \mathrm{~A}$ variação do ângulo $\theta$ entre $\boldsymbol{B}$ e a direção de crescimento é frequentemente usada para
} 
Tabela 6.1: Características do poço quântico digital magnético de (Cd,Mn)Te da Ref. [21]. Aqui estão listadas as dimensões do poço quântico e as concentrações de seus constituintes. Também são apresentadas as faixas de campo magnético e temperatura usadas nos experimentos, bem como os resultados de algumas grandezas que caracterizam da amostra.

\begin{tabular}{|c|c|c|c|}
\hline Parâmetro & Símbolo & Valor & Observação \\
\hline \multicolumn{4}{|c|}{ Configuração do poço quântico digital magnético, Fig. 6.1 } \\
\hline Largura do poço & $L$ & $100 \AA$ & \\
\hline Largura do espaçador & $L_{S}$ & $200 \AA$ & \\
\hline Largura da região dopada & $L_{N}$ & $100 \AA$ & \\
\hline Número de barreiras de MnTe & $N_{b}$ & 3 & \\
\hline Concentração molar de Mg & $y$ & 0.2 & \\
\hline \multicolumn{4}{|l|}{ Condições experimentais } \\
\hline Campo magnético & $B$ & $(0-18) \mathrm{T}$ & \\
\hline Temperatura & $T$ & $(0.03-8.0) \mathrm{K}$ & \\
\hline \multicolumn{4}{|l|}{ Caracterização da amostra } \\
\hline Densidade bidimensional & $n_{s}$ & $(1.6-3.6) \times 10^{11} \mathrm{~cm}^{-2}$ & Baixo campo \\
\hline Mobilidade & $\mu$ & $1.6 \times 10^{4} \mathrm{~cm}^{2} / \mathrm{V} \mathrm{s}$ & Baixo campo \\
\hline Concentração efetiva de MnTe & $x_{e f f}$ & 0.0112 & Simulação \\
\hline
\end{tabular}

Magnetotransporte As medidas de magnetotransporte foram realizadas com o sistema no regime de efeito Hall quântico, como mostram claramente os platôs de $\rho_{x y}$ na Fig. 6.2(a). As oscilações de SdH na resistividade $\rho_{x x}$ refletem a passagem do nível de Fermi nas regiões de estados localizados (vales) e estendidos (picos) do 2DEG. Os principais resultados destas medidas são (i) o aparecimento de um pico de resistividade anômalo em $B_{c} \approx 5.8 \mathrm{~T}$ (fator de preenchimento $\nu \approx 2$ ), e (ii) o comportamento deste pico com os parâmetros externos $\left\{B, \theta, T, n_{s}\right\}$, o que descrevo nos parágrafos seguintes. Os resultados levaram os autores a concluírem que os efeitos observados resultam de uma transição entre estados ferromagnéticos dos elétrons itinerantes.

A Fig. 6.2(b) mostra a resistividade longitudinal na região próxima ao campo crítico para várias temperaturas e com $n_{s}=2.97 \times 10^{11} \mathrm{~cm}^{-2}$. O pico é muito bem definido, separado da oscilação de $\mathrm{SdH}$ normal ${ }^{4}$ para $T=0.33 \mathrm{~K}$ e $1.3 \mathrm{~K}$, sendo máximo nesta última temperatura. Já para a temperatura de $T=2 \mathrm{~K}$ ele é bem menos acentuado e mistura-se com SdH. A $T=8 \mathrm{~K}$ o pico

controlar o cruzamento dos níveis de Landau [e.g. 29, 30, 32, 34]. Aqui Jaroszyński et al. [21] utilizam o efeito Zeeman gigante presente em DMHs para aumentar a polarização do 2DEG e fazer que LLs cruzem em campos moderados. A variação de $\theta$ permite uma variação em torno desta configuração.

${ }^{4} \mathrm{O}$ efeito Shubnikov-de Haas (SdH) refere-se às oscilações de $\rho_{x x} \operatorname{com} B$. Para diferenciar o pico que aparece fora da sequência usual, costuma-se chamá-lo de pico anômalo e referir-se às demais oscilações como SdH usual, ou simplesmente SdH. Note, porém, que quando há estados estendidos no nível de Fermi há condução e, consequentemente, resistividade $\rho_{x x}$. Neste sentido, o pico anômalo não deixa de ser uma oscilação de SdH. 

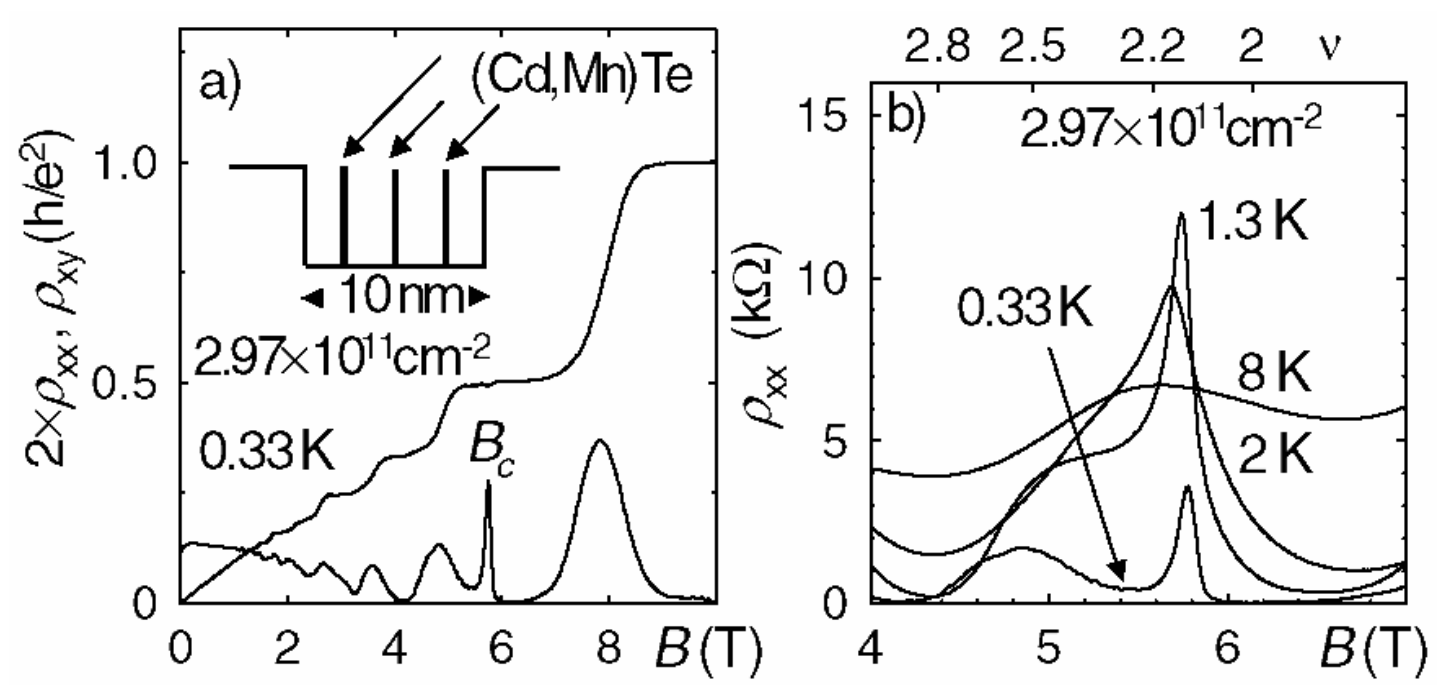

Figura 6.2: Resultados experimentais para magnetotransporte em um poço quântico digital magnético de $(\mathrm{Cd}, \mathrm{Mn}) \mathrm{Te}$ [21, Fig. 1]. As resistividades $\rho_{x x}$ e $\rho_{x y}$ no painel (a) foram medidas a $T=0.33 \mathrm{~K}$ e $n_{s}=2.97 \times 10^{11} \mathrm{~cm}^{-2}$. O pico anômalo de resistividade que ocorre em $B_{c}=5.8 \mathrm{~T}$ é mostrado em (b) para várias temperaturas. Inset: diagrama com o perfil de potencial da banda de condução, com destaque para as barreiras magnéticas. Extraído de Jaroszyński et al. [21].

anômalo não aparece, restando apenas a oscilação de SdH usual.

Pico anômalo $\mathrm{O}$ comportamento de $B_{c}$ em função do ângulo $\theta$ é ilustrado nas Figs. 6.3(a) e (b). Enquanto a interação de troca $s-p$ e o efeito Zeeman ordinário dependem apenas da intensidade do campo $\boldsymbol{B}$, a energia de cíclotron, e consequentemente o espaçamento entre LLs, depende da componente do campo magnético na direção de confinamento do $2 \mathrm{DEG}$, portanto, $\propto B \cos \theta .^{5}$ Ao contrário das oscilações de $\mathrm{SdH}$, que não mudam de posição no gráfico de $\rho_{x x} \times B \cos \theta$ [Fig. 6.3(a)], o pico de resistividade anômalo desloca-se para campos mais baixos com o aumento do ângulo. O gráfico na Fig. 6.3(b) mostra como $B_{c} \cos \theta$ varia com o ângulo $\theta$, com valores praticamente iguais para diferentes densidades. Estas características indicam que a origem deste pico é distinta das oscilações de SdH usuais. ${ }^{6}$

A Fig. 6.3(c) ilustra como o valor do campo magnético do pico anômalo diminui em função da temperatura. Esta diminuição, que também ocorre

\footnotetext{
${ }^{5}$ Esta é uma boa aproximação a menos que $\theta \sim \pi / 2$ [158, págs. 317-325]. De fato, as correções associadas ao ângulo $\theta$ são importantes quando há mais de uma sub-banda envolvida, que não é o caso aqui.

${ }^{6} \mathrm{No}$ efeito $\mathrm{SdH}$, os campos magnéticos correspondentes aos picos de $\rho_{x x}$ (i) aumentam com $n_{s}$ e (ii) não variam com o ângulo $\theta$. O primeiro ponto pode ser entendido lembrando que cada pico de $\mathrm{SdH}$ está associado a um fator de preenchimento $\nu=n_{s} /(e B / h)$; se $n_{s}$ aumenta, o campo magnético correspondente deve ser maior. Já o segundo ponto, quando se varia o ângulo, as posições dos LLs variam na mesma proporção que as respectivas degenerescências,i.e., com $B \cos \theta$; assim a posição dos picos não varia com o ângulo.
} 


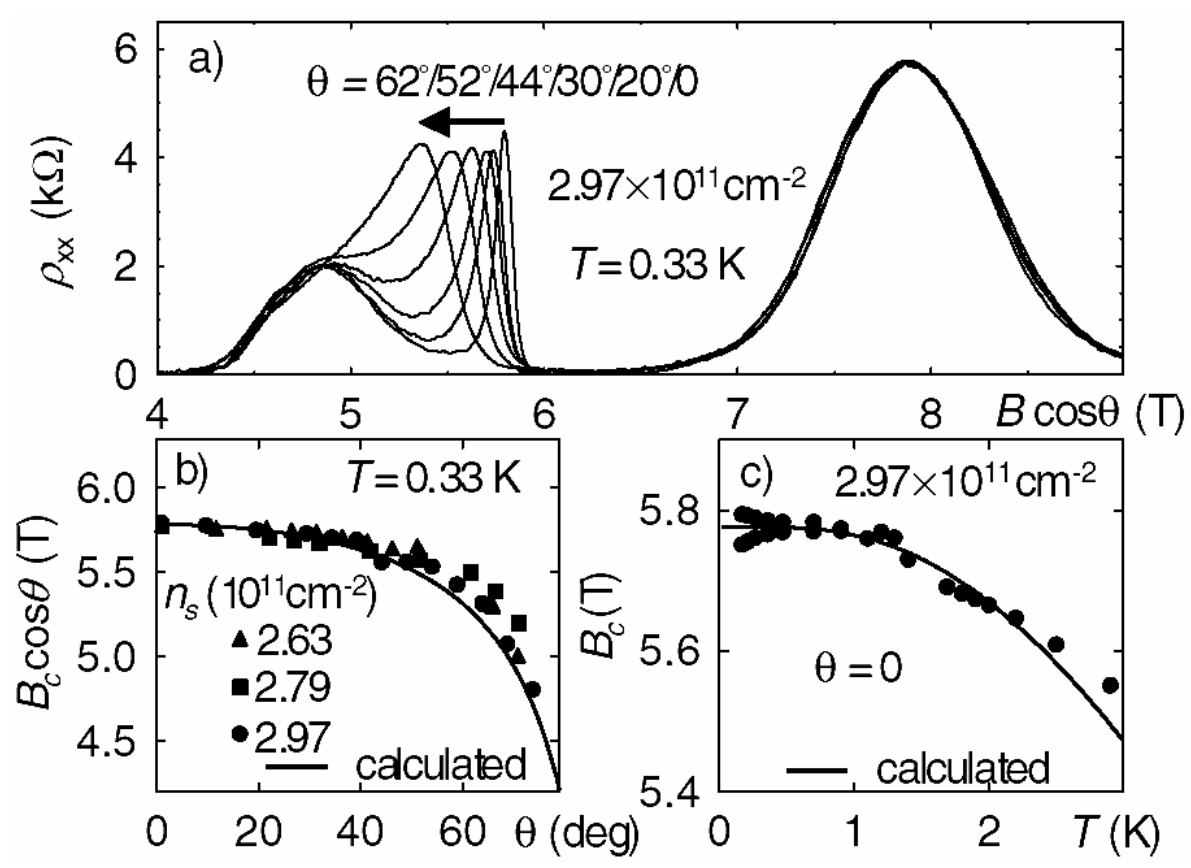

Figura 6.3: (Fig. 2 da Ref. [21].) (a) Resistência $\rho_{x x}$ para $n_{s}=2.97 \times 10^{11} \mathrm{~cm}^{2}$ para vários ângulos $\theta$ entre o campo magnético e a direção de crescimento da amostra. Valores experimentais e calculados [Eq. (6.1)] para a posição dos picos em função de $\theta$ (b) e $T$ (c). Em (c) estão valores experimentais para as duas direções de variação do campo magnético, sendo que os dois ramos a baixas temperaturas refletem o efeito de histerese ilustrado na Fig. 6.5(a).

para as oscilações de SdH usuais, é semelhante àquela observada no DMQW de Knobel et al. [37], discutida no capítulo anterior. Note a presença de dois ramos em $T \rightarrow 0$; eles são para campos magnéticos crescendo e decrescendo, sendo um indicativo de que ocorre histerese neste pico anômalo.

Modelo para os níveis de Landau Para entender estes resultados, e mostrar que o pico anômalo ocorre no cruzamento de dois LLs de diferentes sub-bandas de spin, Jaroszyński et al. [21] utilizaram um modelo simples para a estrutura de níveis de Landau - semelhante ao modelo de Knobel et al. [37] apresentado no Cap. 5. Em uma DMH, a energia de cada LL é dada pela soma da energia de cíclotron, o efeito Zeeman ordinário e a interação de troca s-d [48], i.e.,

$$
\varepsilon_{n}^{\sigma_{z}}=\left(n+\frac{1}{2}\right) \hbar \frac{e B}{m} \cos \theta+\frac{\sigma_{z}}{2}\left[g^{*} \mu_{B} B+x_{e f f} N_{0} \alpha S B_{5 / 2}\left(\frac{g_{M n} \mu_{B} B}{k_{B}\left(T+T_{0}\right)}\right)\right],
$$

na qual $n$ é o índice da sub-banda de Landau, $m$ é a massa efetiva, $g^{*}$ é o fator de Landé efetivo no CdTe, e $N_{0} \alpha$ é a constante da interação de troca $s-d$ (veja os valores na Tabela 6.2 , pág. 145). $B_{5 / 2}$ é a função de Brillouin para spin $S=5 / 2 \mathrm{e}$ $g_{M n}=2.0$. As funções para a concentração efetiva $x_{e f f}(x)$ e temperatura efetiva 


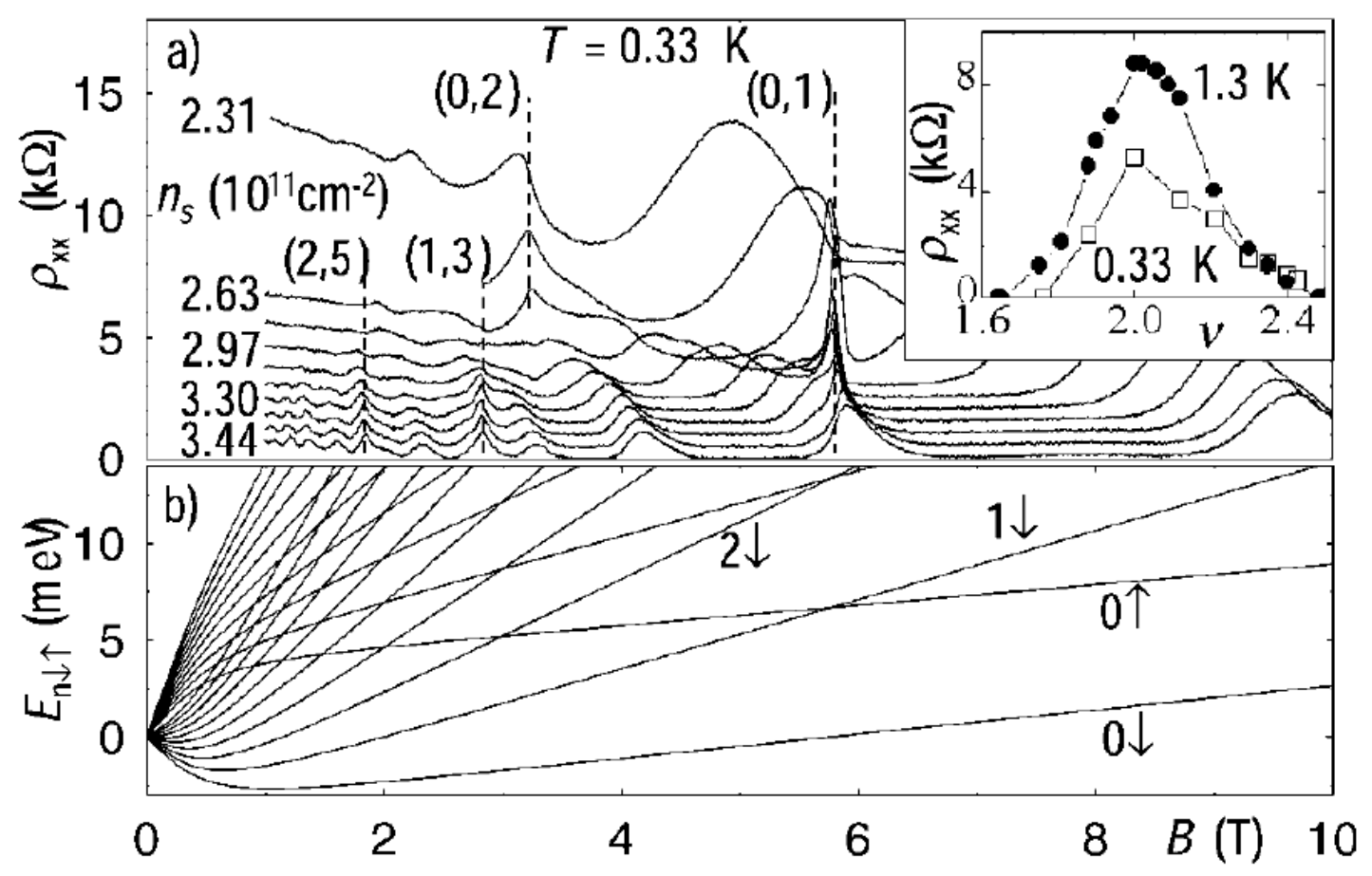

Figura 6.4: (Fig. 3 da Ref. [21].) (a) Resistividade $\rho_{x x}$ a $T=0.33 \mathrm{~K}$ para $n_{s}=$ $2.31-3.44 \times 10^{11} \mathrm{~cm}^{-2}$. As linhas tracejadas marcam os picos e resistividade nos cruzamentos de LLs com índices $(2,5),(1,3),(0,2)$ e $(0,1)$, determinados usando o diagrama de níveis de Landau em (b). O inset mostra o altura do pico em função do fator de preenchimento $\nu$.

$T_{0}(x)>0$ utilizadas foram aquelas parametrizadas por Gaj et al. [59] [Eqs. (2.2) e (2.3)] e levam em consideração a redução da magnetização dos íons de Mn devido a interações antiferromagnéticas.

O modelo da Eq. (6.1), além de não considerar a autoconsistência inerente de sistemas dopados, é para elétrons independentes e não inclui a interação de muitos corpos (troca-correlação) presente no sistema. De fato, utilizando apenas a Eq. (6.1), não foi possível determinar um diagrama de níveis de Landau, como o da Fig. 6.4(b), que indicasse corretamente onde ocorreriam picos anômalos e picos de SdH. A solução foi considerar a contribuição de muitos corpos, o que os autores fizerem utilizando uma correção fenomenológica para a energia de troca (de muitos corpos) determinada experimentalmente nas Refs. [22, 154], dada por

$$
\delta \varepsilon[\mathrm{K}]=2.32+1.79 B \cos \theta
$$

em unidades de temperatura, sendo $B$ em Tesla. Para o caso correspondente ao pico em $B_{c}=5.8 \mathrm{~T}$, o cruzamento de LLs ocorre quando

$$
\varepsilon_{1}^{\downarrow}-\varepsilon_{0}^{\uparrow}=\delta \varepsilon .
$$




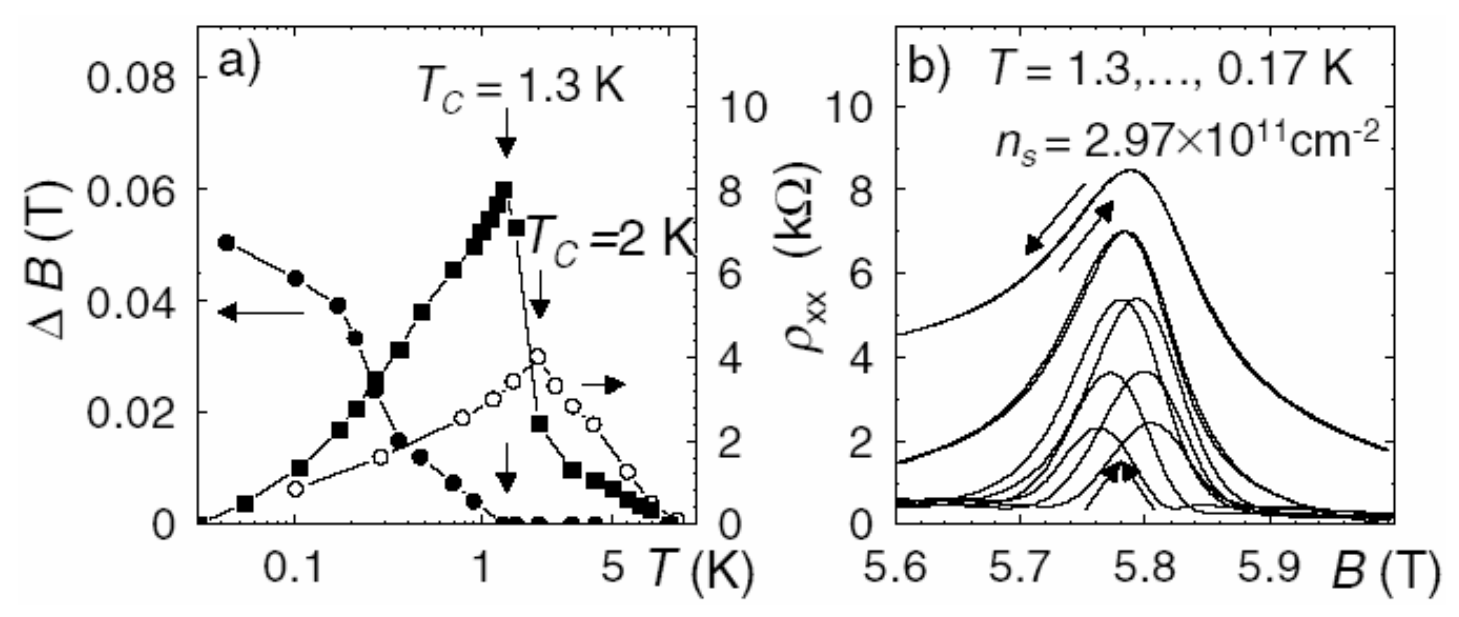

Figura 6.5: (Fig. 4 da Ref. [21].) (a) Altura dos picos em função de $T$ para $n_{s}=2.97 \times 10^{11} \mathrm{~cm}^{-2}$ (quadrados) e $n_{s}=2.54 \times 10^{11} \mathrm{~cm}^{-2}$ (círculos abertos) . Se o pico anômalo e de SdH se sobrepõe, a contribuição deste é subtraída. Os círculos preenchidos mostram a largura dos loops de histerese apresentados em (b), no qual o campo magnético varia na taxa de $0.3 \mathrm{~T} \mathrm{mn}^{-1}$. O comportamento do pico e a presença de histerese magnética são evidências de uma transição de fase para o estado QHF.

Levando em conta esta correção, Jaroszyński et al. [21] fizeram o diagrama de níveis de Landau da Fig. 6.4(b). Este diagrama auxilia associar os picos anômalos (que também ocorrem para outros valores de campo magnético) com os correspondentes cruzamentos de LLs. Utilizando a Eq. (6.1), juntamente com a correção devido energia de troca, e apenas $x_{e f f}$ e $T_{0}$ como parâmetros ajustáveis, foi possível reproduzir o comportamento do campo crítico em função de $\theta$ e $T$, Figs. 6.3(b) e (c), respectivamente.

Um aspecto característico da ocorrência de QHF é que ele é mais acentuado quando o fator de preenchimento $\nu$ for inteiro - a interação de troca é máxima, favorecendo a mesma orientação para os spins [155]. Neste sentido, é sugestivo o resultado para a amplitude do pico anômalo como função de $\nu$, inset da Fig. 6.4(a), com um máximo justamente para $\nu$ inteiro. Este resultado sugere uma relação entre a existência do pico anômalo e a ocorrência de QHF. Entretanto, vale lembrar o experimento analisado no capítulo anterior não apresentou indícios de QHF, apesar da presença do pico anômalo na resistividade.

Histerese Ao variar o campo magnético externo, o pico anômalo desenvolve em campos diferentes, dependendo se o campo magnético aumenta ou diminui. Os dados experimentais mostrando esta histerese estão na Fig. 6.5(b) para diferentes temperaturas. A amplitude $\Delta B$ da histerese - distância entre os picos com $B$ crescendo e diminuindo - diminui com a temperatura [eixo esquerdo do painel (a) da mesma figura], praticamente se anulando na temperatura deno- 
minada $T_{c}$ (diferente para cada densidade $n_{s}$ ). Ao mesmo tempo, a amplitude do pico anômalo aumenta com a temperatura, atinge o máximo em $T_{c}$, e depois diminui. Como a histerese ocorre para $T<T_{c}$ e o pico anômalo é máximo nesta temperatura particular, Jaroszyński et al. [21] identificaram o fenômeno como sendo uma transição de fase em que a temperatura crítica é $T_{c} \cdot{ }^{7}$

Interpretação: ferromagnetismo de efeito Hall quântico Como o efeito observado ocorre com o 2DEG no regime de efeito Hall quântico, é máximo para $\nu$ inteiro, tem uma temperatura crítica $T_{c}$, e sofre um ciclo de histerese para $T<T_{c}$, Jaroszyński et al. [21] analisaram os resultados sob a ótica do fenômeno de ferromagnetismo de efeito Hall quântico (QHF).

Para $B=B_{c}$, ocorre uma transição de fase para o estado fundamental de QHF quando $T<T_{c}$, com os portadores preenchendo apenas uma das subbandas, seja $\uparrow$ ou $\downarrow$. A energia de troca tem um papel fundamental para a formação deste estado. O princípio de exclusão de Pauli determina que a função de onda seja anti-simétrica, fazendo que os elétrons com o mesmo spin não se sobreponham, e diminuindo a energia de Coulomb. Este efeito é mais acentuado quanto maior for a polarização do gás de elétrons. Assim, a interação de troca favorece o alinhamento ferromagnético. ${ }^{8}$

Como o sistema é macroscópico, com os elétrons distribuídos em um plano quase-bidimensional, as variações de potencial definem, localmente, qual das sub-bandas de spin é preenchida. Assim, acredita-se que há formação de domínios ferromagnéticos no 2DEG, cujas fronteiras funcionam como canais de condução ("edge-like channels") [23, 154]. A existência destes canais daria origem a uma condução adicional que, por sua vez, seria responsável pelo pico de resistividade em $B_{c}$. Esta é a descrição geralmente utilizada na literatura para explicar os fenômenos de transporte observados no regime de QHF [20, 154].

Se não ocorresse a formação de estados de QHF, o cruzamento de LLs seria entre dois níveis degenerados e os portadores seriam distribuídos entre eles. Neste caso, que ocorre aqui apenas quando $T>T_{c}$, apareceria um pico de SdH adicional, como observado por Knobel et al. [37] e Harris et al. [38] (Cap. 5), mas sem as assinaturas de ferromagnetismo.

Para certificarem que o fenômeno observado não teria outra origem,

\footnotetext{
${ }^{7}$ Quando se varia a temperatura o sistema eletrônico é ferromagnético para $T<T_{c}$. Esta é uma transição de fase ordinária pois o parâmetro de ordem é a temperatura, i.e., a transição ordem-desordem está associada a flutuações térmicas. Já quando $T<T_{c}$ ocorre uma transição de fase entre dois estados de QHF [25]. Esta é uma transição de fase quântica [159-161], cujo parâmetro de ordem é o campo magnético. O própria transição entre platôs no efeito Hall quântico, inteiro e fracionário, é considerada por alguns autores como uma transição de fase quântica [159]. Nestas transições, as flutuações de caracter quântico é que são importantes.

${ }^{8}$ A Fig. 4.3, na pág. 84, é uma ilustração quantitativa deste efeito.
} 
Jaroszyński et al. [21] fizeram estimativas sobre o ganho de energia livre associado à formação de estados ferromagnéticos [155]. No caso da interação de troca $s$-d entre os íons de Mn e os elétrons, a energia livre é $J \approx 0.02 \mathrm{~K}$ a $T=2 \mathrm{~K}$ e $B=5.8 \mathrm{~T}$, insuficiente para explicar os valores obtidos para $T_{c}$. Por outro lado, uma estimativa Hartree-Fock, seguindo a Ref. [155], fornece um ganho de energia de $J \approx 12 \mathrm{~K}$ a $18 \mathrm{~K}$, mostrando que a interação de troca é capaz de estabilizar o estado de QHF. ${ }^{9}$ Esta energia de troca é cerca de 10 vezes maior que energias de anticrossing $(\approx 1.8 \mathrm{~K})$, devido a interação spin-órbita, observada em sistemas semelhantes [162].

Jaroszyński et al. [21] notam também que, se a energia $J$ for da ordem, ou menor, que a energia associada à desordem intrínseca do sistema, a transição de fase para o estado de QHF não ocorre [163]. Na presença de um potencial de desordem de curto alcance, a teoria [163] prediz que o estado QHF ocorre apenas se $J>\Gamma$, sendo $\Gamma=\hbar\left(\pi e \omega_{c} / 2 \mu m\right)^{1 / 2} / 4 k_{B} \approx 8 \mathrm{~K}$ o alargamento devido a desordem. De fato este valor está consistente com o valor para $J$ calculado pelos autores usando Hartree-Fock. ${ }^{10}$ É importante salientar que, até o momento, os únicos cálculos de fato feitos para este sistema foram estas estimativas de escalas de energia.

Os resultados experimentais de Jaroszyński et al. [21] demonstraram que o efeito Zeeman gigante da interação de troca s-d entre íons magnéticos e os elétrons da banda de condução permitiu alinhar LLs de spins opostos. Com isso foi possível observar o ferromagnetismo de efeito Hall quântico. O modelo apresentado pelos autores reproduz parcialmente os dados experimentais, ${ }^{11}$ mas não fornece nenhuma característica associada com o ferromagnetismo, e.g., histerese ou temperatura crítica. Pelo fato das estimativas de escalas de energia serem consistentes com as teorias sobre a formação de domínios e barreiras entre domínios [154, 157, 164], os autores afirmam que os resultados são uma forte evidência a favor da hipótese da formação domínios. Uma análise mais cuidadosa diria que as evidências são circunstanciais, apesar de plausíveis. Em particular, na análise teórica que eu apresento na próxima seção, eu reproduzo estes resultados experimentais, inclusive a histerese, sem considerar a formação de domínios. Porém, a formulação não impõe nenhum impedimento para que, de fato, os domínios

\footnotetext{
${ }^{9}$ Não há contradição no fato de $J \sim 12 \mathrm{~K}$ enquanto $T_{c} \sim 1 \mathrm{~K}$. Jungwirth e MacDonald [154] recentemente sugeriram que $T_{c}$ pode ser bem menor que $J$, pois é determinada pela competição entre a contribuição da entropia dos domínios magnéticos e o custo energético associado à formação das barreiras entre domínios.

${ }^{10}$ Esta pode ser uma explicação de que porque QHF não apareceu nos experimentos da Refs. [37, 38], Cap. 5. Naquele sistema a desordem é maior, como sugerem os valores da mobilidade e alargamento $\Gamma$.

${ }^{11}$ O modelo das Eqs. (6.1)-(6.3) reproduz a posição de cruzamento de LLs e o comportamento com o ângulo $\theta$ e com a temperatura $T$.
} 
Tabela 6.2: Parâmetros do poço quântico digital magnético de (Cd,Mn)Te da Ref. [21]. A primeira parte da tabela contem valores da literatura para várias propriedades dos materiais utilizados na DMH. A segunda parte lista os valores dos parâmetros que usei nas simulações.

\begin{tabular}{llrc}
\hline \hline Parâmetro & Símbolo & Valor & Obs./Refs. \\
\hline Dados relevantes sobre o material & & & \\
Massa efetiva & $m / m_{0}$ & 0.099 & {$[49]$} \\
Constante dielétrica estática & $\epsilon / \epsilon_{0}$ & 10.0 & {$[49]$} \\
Parâmetro de rede & $a$ & $6.4835 \AA$ & {$[49]$} \\
Fator de Landé efetivo & $g$ & -1.67 & {$[21]$} \\
Constante de troca s- $d$ & $N_{0} \alpha$ & $220 \mathrm{meV}$ & {$[45]$} \\
gap $\mathrm{Cd}_{1-y} \mathrm{Mg}_{y} \mathrm{Te}$ & $E_{g a p}^{\text {CdMgTe }}$ & $(1.606+1.578 y) \mathrm{eV}$ & {$[49]$} \\
gap $\mathrm{Cd}_{1-x} \mathrm{Mn}_{x} \mathrm{Te}$ & $E_{g a p}^{\text {CdMnTe }}$ & $(1.606+1.654 x) \mathrm{eV}$ & {$[165]$} \\
Offset banda condução & $C B O$ & 0.75 & {$[49]$} \\
\hline Parâmetros nas simulações & & & \\
Offset na interface CdMgTe/CdTe & $v_{0}$ & $248.1 \mathrm{meV}$ & \\
Offset na interface CdTe/CdMnTe & $v_{b}$ & $1183.5 \mathrm{meV}$ & \\
Densidade bidimensional & $n_{s}$ & $2.97 \times 10^{11} \mathrm{~cm}{ }^{-2}$ & \\
Concentração planar de MnTe & $x_{p}$ & 0.115 & \\
Temperatura efetiva & $T_{0}$ & $0.47 \mathrm{~K}$ & \\
Função de perfil para MnSe & $\wp(z)$ & $\wp \mathrm{exp}(z)$ & Eq. $(2.24)$ \\
Comprimento de uma monocamada & $d$ ou ML & $3.24 \AA$ & $d=a / 2$ \\
Comprimento característico & $\ell$ & $4.67 \AA$ & $1.44 \mathrm{ML}$ \\
Alargamento DOS & $\Gamma$ & $0.36 \mathrm{meV}$ & \\
Alargamento DOS estados extendidos & $\Gamma_{e x t}^{\uparrow}$ & $0.25 \mathrm{meV}$ & \\
& $\Gamma_{e x t}^{\downarrow}$ & $0.25 \mathrm{meV}$ & \\
\hline \hline
\end{tabular}

existam e sejam, possivelmente, importantes para descrever microscopicamente os estados de QHF e a formação de picos de resistividade.

\subsection{Resultados teóricos}

Nesta seção eu utilizo o modelo descrito no Cap. 4 para investigar o poço quântico digital magnético ilustrado na Fig. 6.1. Eu calculo a estrutura eletrônica na aproximação da massa efetiva resolvendo as equações autoconsistentes de Kohn-Sham. Utilizando uma densidade de estados com alargamento gaussiano, eu então calculo várias propriedades do sistema, a saber, densidades, magnetotransporte e energia total. Veja o Quadro 2 na Pág. 108 para um resumo das principais equações de trabalho. As simulações foram efetuadas usando os parâmetros apresentados na Ref. [21] (Tabela 6.1) e valores típicos encontrados na literatura (Tabela 6.2) para outras propriedades utilizadas no modelo. 


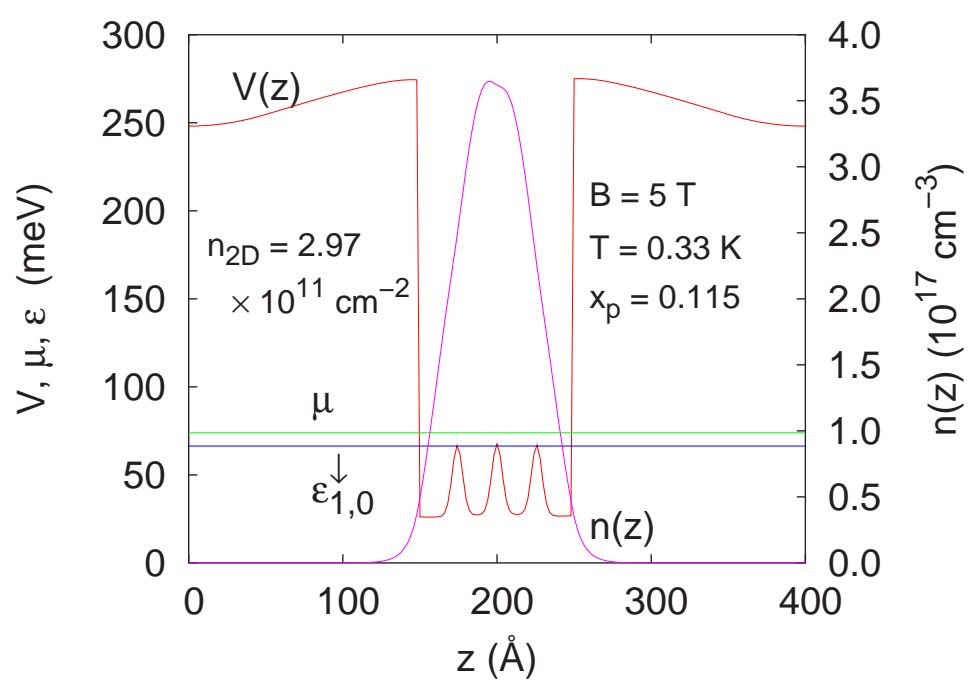

Figura 6.6: Perfil de potencial autoconsistente, resultado do cálculo da estrutura eletrônica do poço quântico digital magnético da Fig. 6.1. As três regiões contendo Mn foram modeladas usando um perfil característico de interdifusão. Também são apresentados a energia do nível de Landau $|0, \downarrow\rangle$ da primeira sub-banda, o potencial químico $\mu$ e o perfil de densidade $n(z)$ do 2DEG confinado no poço quântico.

Perfil de potencial Os elétrons na banda de condução sentem um potencial efetivo composto de diversas contribuições, Eq. (4.77),

$v_{\text {eff }}^{\sigma_{z}}\left(z ; B, T,\left[n_{\uparrow}, n_{\downarrow}\right]\right)=\underbrace{v_{0}(z)+v_{b}(z)}_{\text {Estrutural }}+\underbrace{v_{\text {sp-d }}^{\sigma_{z}}(z ; B, T)}_{\text {Troca sp-d }}+\underbrace{v_{h}(z ;[n])}_{\text {Hartree }}+\underbrace{v_{x c}^{\sigma_{z}}\left(z ;\left[n_{\uparrow}, n_{\downarrow}\right]\right)}_{\text {Troca-correlação }}$.

A parte estrutural consiste dos offsets na banda de condução devido às interfaces da heteroestrutura. Utilizando os valores para as energias dos gaps indicados na Tabela 6.2, e atribuindo $75 \%$ das diferenças nas interfaces para a banda de condução, a altura do poço quântico ficou em $v_{0}=248.1 \mathrm{meV}$ e a altura das barreiras com $\mathrm{Mn}$ resultou em $v_{b}=1183.5 x(z) \mathrm{meV}$, sendo $x(z)$ a concentração nominal de Mn.

Para a contribuição de troca $s$ - $d$ eu utilizei a constante de troca do (Cd,Mn)Te, $N_{0} \alpha=0.22 \mathrm{eV}$ [45], e o modelo de aglomerados com singletos e tripletos para a concentração efetiva de Mn (Cap. 2). Desta vez eu utilizei um perfil de interdifusão para simular a concentração de Mn ao longo da amostra. ${ }^{12}$ Da mesma forma que no capítulo anterior, eu fixei o comprimento característico do perfil de Mn e ajustei a concentração planar $x_{p}$ que melhor ajustava os resultados experimentais. A concentração obtida foi $x_{p}=0.115$, que corresponde uma concentração efetiva $x_{e f f}=0.009$ [cf. Eq. (2.32)], relativamente próximo ao valor

\footnotetext{
${ }^{12}$ Como discutido no Cap. 2, tanto o perfil de interdifusão (função erro), quanto segregação (exponencial), apresentam resultados muito próximos.
} 

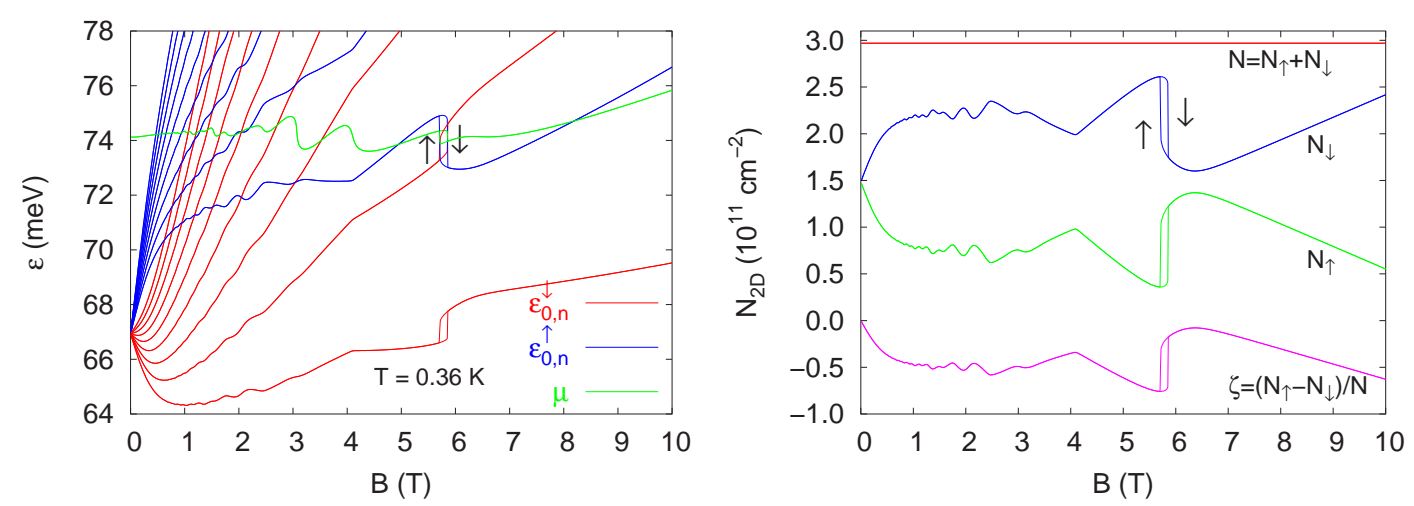

Figura 6.7: O diagrama de níveis de Landau (a) mostra como a interação de troca $s$-d acarreta um desdobramento de spin gigante que, por sua vez, faz com que os níveis $|0, \uparrow\rangle$ e $|1, \downarrow\rangle$ cruzem em $B_{c} \simeq 5.7 \mathrm{~T}$. Resultados para $B$ crescendo e diminuindo estão sobrepostos, sendo que em $B_{c}$ há um comportamento de histerese (as setas próximas às regiões com histerese indicam o sentido de varredura do campo $B$ em cada ramo). As densidades bidimensionais parciais e a polarização $\zeta$ oscilam à medida que a ocupação dos níveis de Landau varia com o campo magnético (b). Em $B_{c}$ a histerese também ocorre no gráfico das densidades.

estimado por Jaroszyński et al. [21] de 0.0112.

O potencial de Hartree e de troca-correlação são calculados autoconsistentemente, o primeiro via a equação de Poisson e o segundo usando a parametrização de Vosko, Wilk, e Nusair [99] para o potencial XC na aproximação LSDA da DFT. Este último ingrediente é essencial para reproduzir os efeitos relacionados com ferromagnetismo itinerante observados experimentalmente [21] e que foram apresentados na seção anterior. O perfil de potencial resultante está ilustrado na Fig. 6.6.

Estrutura eletrônica Resolvendo as equações autoconsistentes de Kohn-Sham para os elétrons na banda de condução, Eq. (4.19),

$$
\left[-\frac{\hbar}{2 m} \frac{\partial^{2}}{\partial z^{2}}+v_{e f f}^{\sigma_{x}}\left(z ; B, T,\left[n_{\uparrow}, n_{\downarrow}\right]\right)\right] \chi_{i}^{\sigma_{z}}(z)=\varepsilon_{i}^{\sigma_{z}} \chi_{i}^{\sigma_{z}}(z),
$$

eu obtenho estrutura eletrônica do sistema. A energia dos níveis de Landau é escrita como, Eq. (4.49),

$$
\varepsilon_{i, n}^{\sigma_{z}}=\varepsilon_{i}^{\sigma_{z}}+\left(n+\frac{1}{2}\right) \hbar \omega_{c}+\frac{\sigma_{z}}{2} g^{*} \mu_{B} B
$$

A concentração $x_{p}$ de $\mathrm{Mn}$ foi ajustada para que, via o desdobramento de spin gigante devido a interação de troca $s$-d, ocorresse um cruzamento de níveis de Landau em $B=5.7 \mathrm{~T}$, reproduzindo os resultados de Jaroszyński et al. [21] ilustrados na Fig. 6.4. A Figura 6.7 mostra (a) o diagrama de níveis de Landau 
para $T=0.33 \mathrm{~K} \mathrm{e} \mathrm{(b)} \mathrm{as} \mathrm{densidades} \mathrm{bidimensionais,} \mathrm{Eq.} \mathrm{(4.61),}$

$$
n_{2 D}^{\sigma_{z}}=\int_{-\infty}^{\infty} \sum_{i, n} g_{i, n}^{\sigma_{z}}(\varepsilon) f(\varepsilon) \mathrm{d} \varepsilon \quad, \quad n_{2 D}=n_{2 D}^{\downarrow}+n_{2 D}^{\uparrow}
$$

com $g_{i, n}^{\sigma_{z}}(\varepsilon)$ a densidade de estados (4.54) com alargamento gaussiano e $f(\varepsilon)$ a função de Fermi (4.59). Tanto esta figura, quanto as próximas desta seção, mostram a sobreposição dos resultados com o campo magnético $B$ ora aumentando, ora diminuindo de intensidade. O resultado foi que em $B \sim 5.7 \mathrm{~T}$ ocorreu uma histerese na estrutura eletrônica [Fig. 6.7(a)] que se refletiu em todas as demais grandezas calculadas [e.g. Fig. 6.7(b)].

Note a diferença fundamental entre o modelo teórico utilizado por Jaroszyński et al. [21] para explicar os dados experimentais e o modelo aqui proposto. Naquele caso, uma correção fenomenológica é utilizada para considerar o efeito de muitos corpos de troca, Eqs. (6.2) e (6.3). Aqui eu incluo o potencial autoconsistente de troca-correlação, este sim baseado apenas no gás de elétrons interagente. Esta contribuição é dependente de spin, sendo que o desdobramento de spin é mais acentuado à medida que o gás de elétrons fica mais polarizado [99, 100, 112], cf. Fig. 4.3. Este fato nada mais é que o princípio de exclusão de Pauli em ação na forma da interação de troca. No caso do modelo do grupo experimental, a correção devido a troca serviu apenas para "acertar" o desdobramento de spin. Aqui, eu não apenas calculo o desdobramento de spin, mas também obtenho o efeito de histerese ilustrado na Fig. 6.7. A correção da Eq. (6.2) está automaticamente incluída na minha formulação via o potencial de troca-correlação dependente de spin.

De fato, os valores para $x_{\text {eff }}$ confirmam o papel de XC no desdobramento de spin. A concentração efetiva estimada por Jaroszyński et al. [21] usando fotoluminescência foi $x_{e f f}=0.020$ (considera que todo o desdobramento de spin vem da interação de troca $s-d)$. O valor usado no modelo das Eqs. (6.1) e (6.2) foi menor, $x_{e f f}=0.0112$; como aquele modelo inclui uma correção de $\mathrm{XC}$, mesmo que de forma fenomenológica, a contribuição da interação de troca deve ser menor para um mesmo desdobramento de spin total, implicando numa concentração menor de Mn. Já o meu cálculo confirma este resultado, uma vez que $x_{e f f}=0.009$, menor que o valor de fotoluminescência, mas próximo do valor da simulação com a correção de XC feita pelo grupo experimental.

Magnetotransporte Uma vez calculada a estrutura eletrônica e a DOS do sistema, eu simulo o magnetotransporte do 2DEG usando o modelo descrito no Cap. 4. Eu primeiro calculo as componentes do tensor de magneto- 

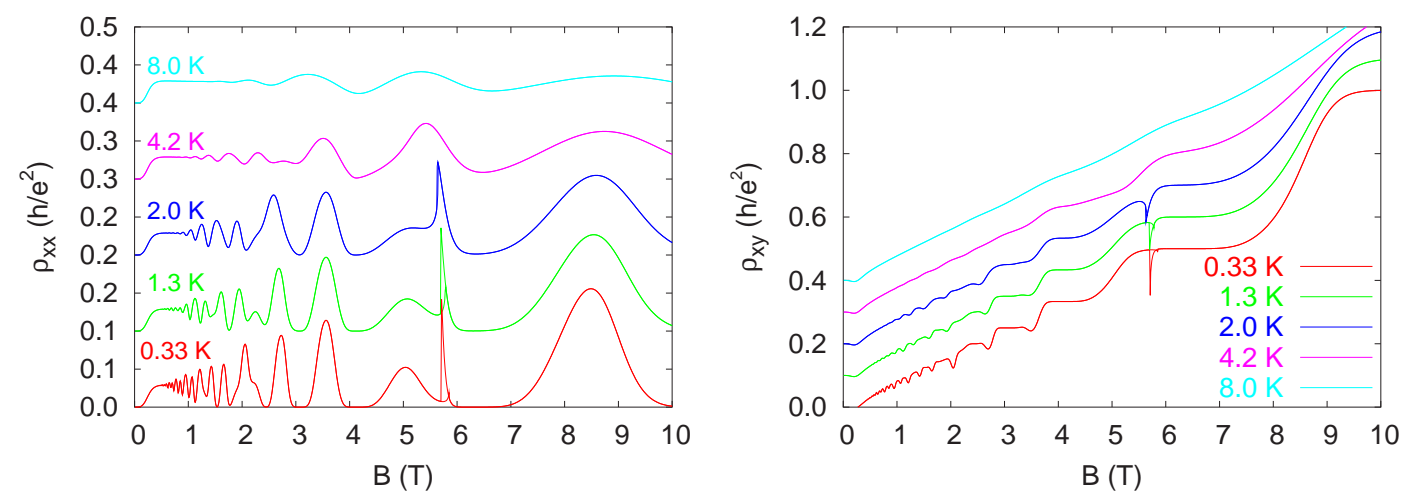

Figura 6.8: Resultados teóricos para o magnetotransporte no poço quântico digital magnético de $(\mathrm{Cd}, \mathrm{Mn}) \mathrm{Te}$. A resistividade $\rho_{x x}$ apresenta as oscilações de Shubnikov-de Haas, sendo que um pico anômalo aparece em $B_{c} \simeq 5.7 \mathrm{~T}$ para as temperaturas até $T=2.0 \mathrm{~K}(\mathrm{a})$. A resistividade $\rho_{x y}$ apresenta os platôs característicos do efeito Hall quântico inteiro (b). Note que, onde ocorre o pico anômalo em $\rho_{x x}$, também ocorre uma depressão em $\rho_{x y}$. Os gráficos são para a densidade $n_{s}=2.97 \times 10^{11} \mathrm{~cm}^{-2}$. Para cada temperatura os gráficos estão deslocadas de $0.1 h / e^{2}$ para facilitar a visualização.

condutividade, Eq. (4.74),

$$
\begin{gathered}
\sigma_{x x}=\frac{e^{2}}{h} \frac{2}{\pi} \int_{-\infty}^{\infty}\left(-\frac{\partial f(\varepsilon)}{\partial \varepsilon}\right) \sum_{i, n, \sigma_{z}}\left(n+\frac{1}{2}\right) \exp \left[-\left(\frac{\varepsilon-\varepsilon_{i, n}^{\sigma_{z}}}{\Gamma_{\mathrm{ext}}^{\sigma_{z}}}\right)^{2}\right] \mathrm{d} \varepsilon \\
\sigma_{x y}=\frac{e n_{2 D}\left[g_{\text {ext }}\right]}{B}+\frac{e^{2}}{h} \frac{2}{\pi} \int_{-\infty}^{\infty}\left(-\frac{\partial f(\varepsilon)}{\partial \varepsilon}\right) \sum_{i, n, \sigma_{z}}\left(n+\frac{1}{2}\right) \frac{\Gamma_{\mathrm{ext}}^{\sigma_{z}}}{\hbar \omega_{c}} \exp \left[-\left(\frac{\varepsilon-\varepsilon_{i, n}^{\sigma_{z}}}{2 \Gamma_{\mathrm{ext}}^{\sigma_{z}}}\right)^{2}\right] \mathrm{d} \varepsilon
\end{gathered}
$$

e depois inverto-o para obter a magnetorresistividade, Eq. (4.76),

$$
\rho_{x x}=\frac{\sigma_{x x}}{\sigma_{x x}^{2}+\sigma_{x y}^{2}} \quad, \quad \rho_{x y}=\frac{\sigma_{x y}}{\sigma_{x x}^{2}+\sigma_{x y}^{2}}
$$

Na Fig. 6.8 eu mostro (a) as oscilações de Shubnikov-de Haas na resistividade $\rho_{x x}$ e (b) a resistividade transversal $\rho_{x y}$ exibindo o efeito Hall quântico.

A magnetorresistividade longitudinal $\rho_{x x}$ apresenta um pico anômalo bastante pronunciado em $B \simeq 5.7 \mathrm{~T}$ nas temperaturas $T=\{0.33,1.3,2.0\} \mathrm{K}$, Fig. 6.8(a). Já para $T \geq 4.2 \mathrm{~K}$ aparece apenas uma oscilação de $\mathrm{SdH}$ usual. O pico ocorre justamente no valor de campo magnético em que há cruzamento de níveis, neste caso os LLs $|0, \uparrow\rangle$ e $|1, \downarrow\rangle$. A Fig. 6.9 mostra uma ampliação da região do pico anômalo revelando dois picos, um para $B$ aumentando e outro para $B$ diminuindo.

Comparando com os resultados experimentais das Figs. 6.2 e 6.5(b), observa-se que, por um lado, (i) as minhas simulações reproduzem tanto o apare- 


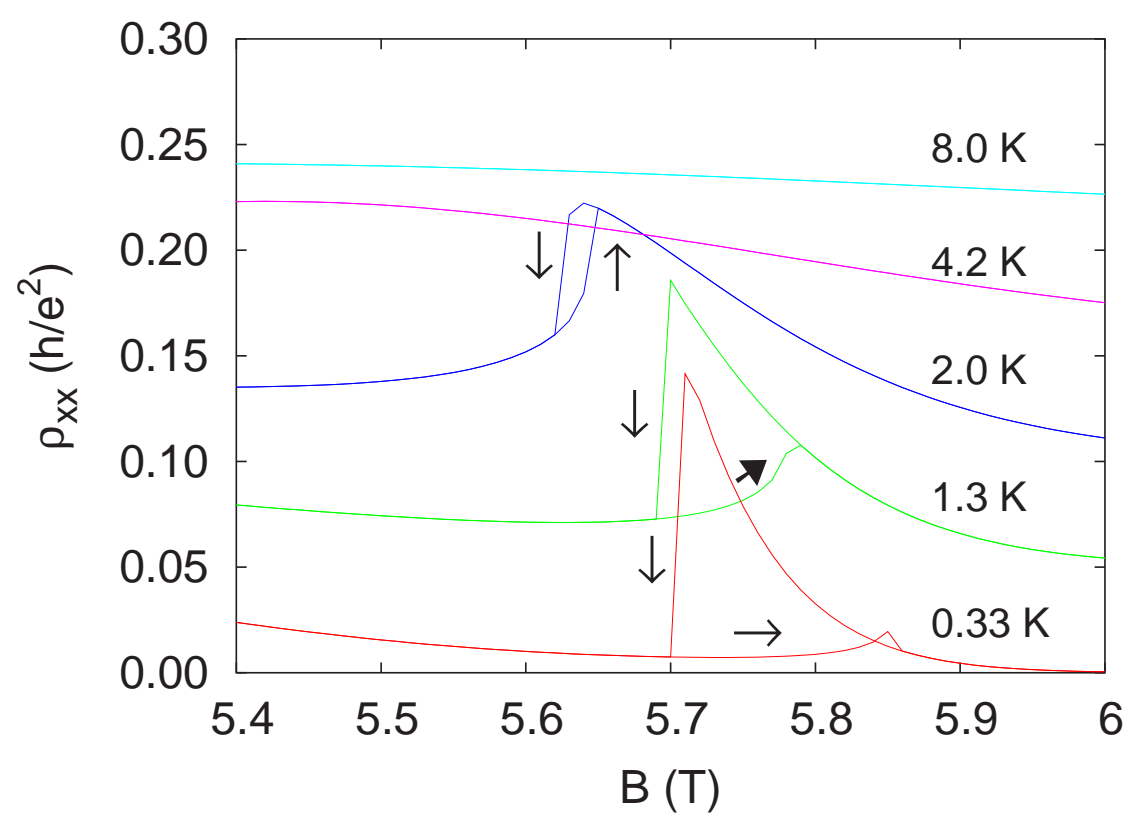

Figura 6.9: Ampliação da Fig. 6.8(a) na região do pico anômalo para diversas temperaturas. As curvas a cada temperatura estão deslocadas de $0.05 h / e^{2}$ e as setas indicam a direção de variação do campo magnético. À medida que a temperatura aumenta, as oscilações de $\mathrm{SdH}$ e o pico anômalo deslocam-se para campos magnéticos mais baixos. Note que, ao contrário dos dados experimentais, os picos anômalos para $B$ aumentando e diminuindo são assimétricos e têm amplitudes diferentes.

cimento de um pico anômalo, quanto a histerese, e por outro lado, (ii) enquanto os dois picos da Fig. 6.5(b) têm a mesma amplitude e são simétricos, os picos da simulação têm amplitudes bem diferentes e são assimétricos. De fato, uma comparação direta não pode ser feita porque, como indicado na Ref. [21], as curvas da Fig. 6.5(b) foram ajustadas subtraindo o que os autores consideraram ser a contribuição de SdH usual para a altura do pico. Os resultados que eu apresento na Fig. 6.9 não têm subtração ou qualquer outro tipo de tratamento de dados. Adicionalmente, como já mencionado, o modelo que eu utilizo para magnetotransporte fornece amplitudes para $\rho_{x x}$ específicas para o limite de espalhamentos de curto alcance (Sec. 4.4).

De qualquer maneira, no cálculo aqui apresentado esta diferença na altura e simetria dos picos está relacionada com a posição relativa dos estados estendidos com relação ao potencial químico. Na simulação, a passagem de $\mu$ sobre os estados estendidos é abruptamente interrompida pela transição entre estados ferromagnéticos, o que explica a assimetria dos picos. No caso do pico maior ( $B$ diminuindo), $\mu$ passa por boa parte dos estados estendidos antes de ocorrer a transição. Já no caso do pico menor ( $B$ aumentando), a transição ocorre um pouco depois que $\mu$ começa a atravessar os estados estendidos do LL, 

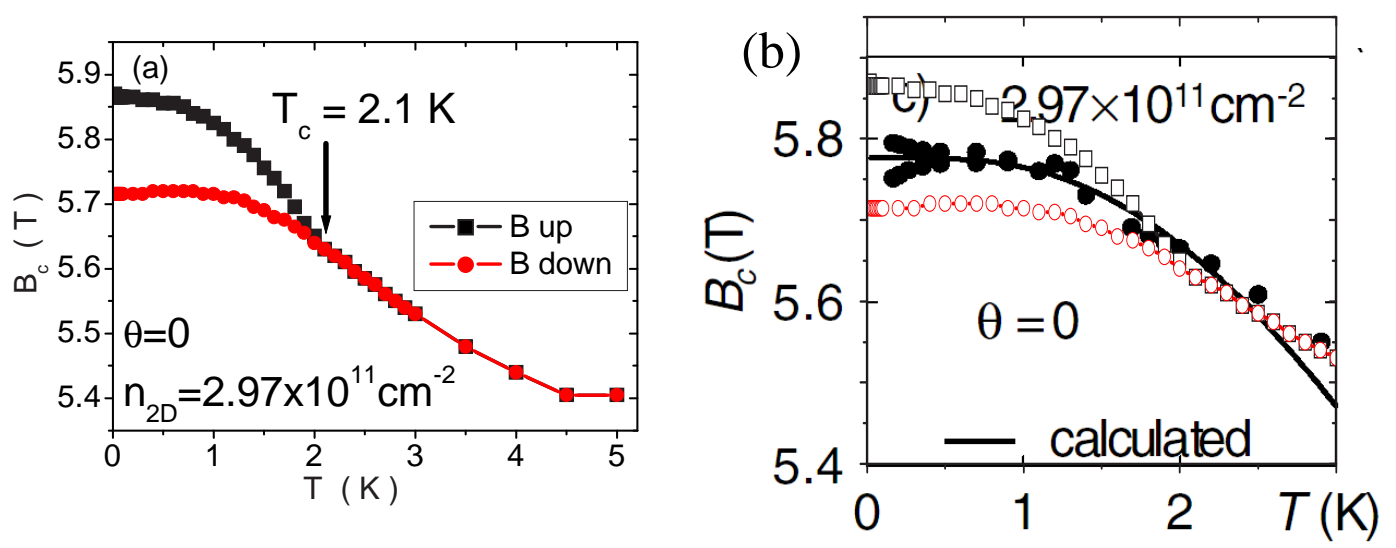

Figura 6.10: Simulação da posição do pico anômalo em função da temperatura $T$ para o campo magnético aumentando e diminuindo (a). À medida que a temperatura aumenta o pico anômalo desloca-se para campos magnéticos mais baixos. O painel (b) mostra os mesmos resultados de (a), mas sobrepostos aos resultados experimentais. Para $T \lesssim 2.1 \mathrm{~K}$ aparece a histerese, enquanto para $2.1 \mathrm{~K}<T<3.5 \mathrm{~K}$ o comportamento é quase linear, mais parecido com os pontos experimentais que a simulação do grupo experimental (linha cheia, calculated).

e por isso o pico é menor. A diferença diminui à medida que $T$ aumenta e as configurações eletrônicas para $B$ aumentando e diminuindo ficam cada vez mais próximas.

Já o gráfico do efeito Hall, Fig. 6.8(b), apresenta uma depressão justamente na região que ocorre o pico anômalo em $\rho_{x x}$. Esta depressão não aparece no gráfico experimental, Fig. 6.2(a). Analisando em termos do modelo para o magnetotransporte, da mesma forma que as regiões com $\rho_{x x}$ (quase-)nulo corresponde aos platôs em $\rho_{x y}$, um pico em $\rho_{x x}$ deve corresponder a uma variação em $\rho_{x y}$. Em outras palavras, o platô ocorre quando o potencial químico está no gap de mobilidade (estados localizados) e $\rho_{x y}$ varia quando $\mu$ passa pelos estados estendidos. Assim, não apenas em termos do modelo que utilizo para o magnetotransporte, mas também em termos do entendimento comum para o efeito Hall quântico inteiro [166-168], não tenho como justificar a ausência de variação de $\rho_{x y}$ experimental quando ocorre um pico em $\rho_{x x}$ em $B_{c}$.

Porém, uma inspeção cuidadosa na curva experimental de $\rho_{x y}$, Fig. 6.2(a), mostra diminutas oscilações próximo de $B_{c}$. Pode ser então que, de fato, esta depressão exista, mas meu resultado esteja superestimando a sua magnitude. De fato, apesar da maioria dos trabalhos experimentais divulgados mostrando o aparecimento do pico anômalo e ciclos de histerese não mostrarem as respectivas curvas de $\rho_{x y}$, posso citar as Refs. $[23,29,32]$ nas quais as curvas de $\rho_{x y}$ apresentam variações nas regiões de campo magnético correspondentes aos picos de $\rho_{x x}$, inclusive quando são faixas de campo em que há cruzamento de LLs. 

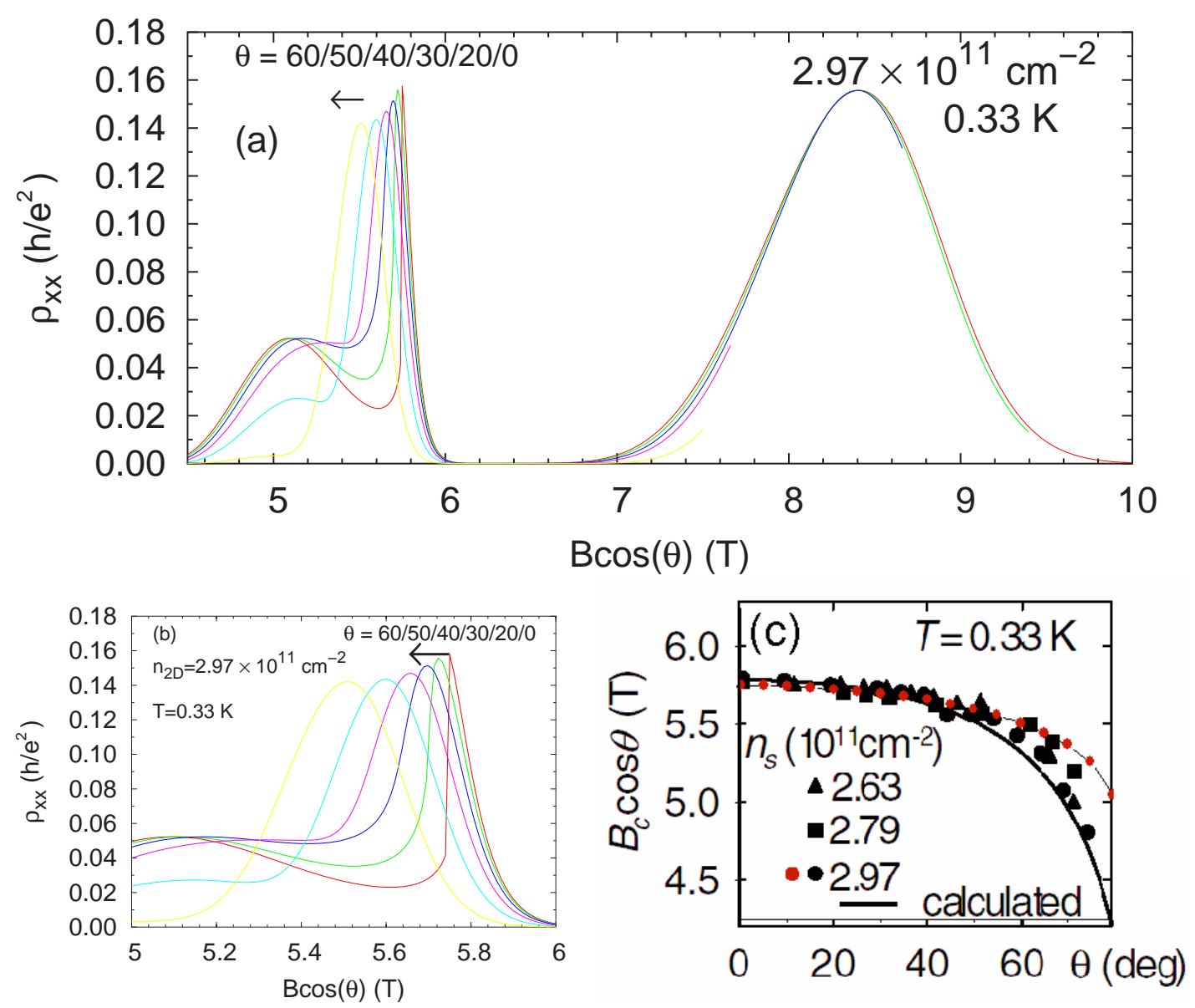

Figura 6.11: Simulação da posição do pico anômalo em função da ângulo $\theta$ do campo magnético com relação a amostra. O painel (a) mostra que apenas o pico anômalo muda de posição quando $\rho_{x x}$ é apresentado em função de $B \cos \theta$. Uma ampliação da região do pico anômalo é apresentada em (b). Em (c) o resultado da minha simulação para $B_{c} \cos \theta$ está sobreposto com o resultado experimental da Fig. 6.3(b). A concordância é relativamente boa, divergindo para ângulos grandes, como era de se esperar. A linha cheia (calculated) refere-se à simulação fenomenológica do grupo experimental.

Pico anômalo: dependência com $T$ Agora eu analiso o comportamento do pico anômalo em função da temperatura $T$ e do ângulo $\theta$. A Fig. 6.10 mostra que as oscilações de $\rho_{x x}$ deslocam-se para campos magnéticos mais baixos à medida que a temperatura aumenta. Este efeito também foi discutido no capítulo anterior ao analisar os resultados de Knobel et al. [37]. Na Fig. 6.10(b) eu comparo a posição do pico anômalo em função da temperatura obtida dos meus cálculos teóricos com os resultados experimentais e teóricos de Jaroszyński et al. [21]. Para $T \lesssim 2.1 \mathrm{~K}$, os meus resultados teóricos abrem-se em dois ramos, para $B$ crescendo e diminuindo, refletindo justamente o efeito de histerese que aparece nos dados experimentais. Nesta simulação teórica, a histerese começa numa temperatura maior e a sua amplitude é aproximadamente o dobro do valor 
experimental. Para $2.1 \mathrm{~K}<T<3.5 \mathrm{~T}$, o comportamento é aproximadamente linear, bastante próximo dos dados experimentais. Note que a curvatura da simulação de Jaroszyński et al. [21],linha cheia na Fig. 6.10(b), reflete apenas a dependência da temperatura da função de Brillouin [cf. Eq. (6.1)]. Em contraste, o modelo que eu utilizo, além conter a temperatura via a função de Brillouin, também inclui a temperatura via a função de Fermi. Adicionalmente, o fato da minha formulação ser autoconsistente permite descrever melhor a estrutura eletrônica do sistema e, consequentemente, a posição dos LLs em função de $B$ e $T$, justificando o bom acordo com os dados experimentais.

Pico anômalo: dependência $\operatorname{com} \theta$ Para calcular o efeito da variação do ângulo do campo magnético com relação a direção de crescimento da amostra, eu considero que o único efeito deste é afetar a quantização de Landau. Neste caso, tanto a frequência de cíclotron quanto a degenerescência de Landau são proporcionais à componente do campo perpendicular à amostra, i.e., $B \cos \theta$ $[158] .{ }^{13}$ As demais grandezas que variam explicitamente com o campo, troca $s-d$ e Zeeman ordinário, não são afetados. A Fig. 6.11 mostra o comportamento do pico anômalo em função do ângulo. O painel (a) mostra que a posição do pico anômalo varia $\operatorname{com} B \cos \theta$ enquanto a oscilação de $\mathrm{SdH}$ permanece na mesma posição, reproduzindo os resultados experimentais. ${ }^{14} \mathrm{O}$ painel (c) mostra os resultados da simulação para o campo $B_{c}$ sobreposto com os resultados experimentais - a concordância é relativamente boa. Lembre que o modelo do grupo experimental precisou utilizar uma dependência fenomenológica explícita com $\theta$ na correção da energia [Eq. (6.2)] para poder reproduzir a dependência de $B_{c} \operatorname{com} \theta$.

Uma análise mais detalhada da estrutura dos níveis de Landau quando ocorre o cruzamento de níveis com spins opostos revela que o sinal do fator giromagnético efetivo $g^{*}$ determina o sentido em que o pico anômalo desloca à medida que se varia o ângulo. Para ilustrar este efeito eu considero as energia dos níveis $|1, \downarrow\rangle$ e $|0, \uparrow\rangle$,

$$
\begin{aligned}
& \varepsilon_{1,1}^{\downarrow}=\varepsilon_{1}^{\downarrow}+\frac{3}{2} \hbar \omega_{c}-\frac{1}{2} g^{*} \mu_{B} B, \\
& \varepsilon_{1,0}^{\uparrow}=\varepsilon_{1}^{\uparrow}+\frac{1}{2} \hbar \omega_{c}+\frac{1}{2} g^{*} \mu_{B} B .
\end{aligned}
$$

As energias de sub-banda $\varepsilon_{1}^{\sigma_{z}}$ contêm as contribuições de troca $s$ - $d$ e da interação de Coulomb (Hartree $+\mathrm{XC}$ ).

No campo $B_{c}$ em que ocorre o cruzamento de LLs tenho que

$$
\varepsilon_{1,1}^{\downarrow}-\varepsilon_{1,0}^{\uparrow}=0
$$

\footnotetext{
${ }^{13}$ Veja a nota 5 na pág. 139.

${ }^{14}$ Veja a explicação para este efeito na Pág. 139.
} 
Substituindo (6.5) em (6.6) tenho

$$
\left(\varepsilon_{1}^{\downarrow}-\varepsilon_{1}^{\uparrow}\right)+\hbar \omega_{c}-g^{*} \mu_{B} B=0 \quad \therefore \quad \hbar \frac{e B}{m} \cos \theta=-\left(\varepsilon_{1}^{\downarrow}-\varepsilon_{1}^{\uparrow}\right)+g^{*} \mu_{B} B .
$$

A diferença das energias das sub-bandas de spin tem duas componentes: (i) o desdobramento de spin devido a interação de troca $s$ - $d, x_{e f f} N_{0} \alpha\left\langle S_{z}\right\rangle$, e (ii) a contribuição proveniente da interação de Coulomb, mais especificamente, da contribuição de troca e correlação dependente de spin, que aqui denoto por $\delta \varepsilon$. Para $B \approx 6 \mathrm{~T}$ em que ocorre o cruzamento de LLs, interação de troca $s$ - $d$ já está praticamente saturada, ou seja,

$$
x_{e f f} N_{0} \alpha\left\langle S_{z}\right\rangle \approx \mathbb{C} \text { onst } .
$$

Já o termo de XC pode ter ou não uma dependência complicada com o campo magnético $B$ e o ângulo $\theta$. Três opções simples seriam

$$
\delta \varepsilon \rightarrow \quad \mathbb{C} \quad, \quad \propto B \quad, \quad \propto B \cos \theta,
$$

ou seja, pode ser constante, diretamente proporcional a $B$, ou proporcional a $B \cos \theta$. Os resultados experimentais de De Poortere et al. [22] indicam que a última opção é mais adequada. Assim, utilizando (6.8) e (6.9), e denotando $A=\hbar e / m+\delta \varepsilon / B \cos \theta$, a Eq. (6.7) fica

$$
A B \cos \theta=\mathbb{C}+\frac{g^{*} \mu_{B}}{\cos \theta} B \cos \theta
$$

A Fig. 6.3 ilustra o comportamento de $B_{c} \cos \theta$ em função do ângulo $\theta$ utilizando a Eq. (6.10). Nos painéis (a) e (c) estão ilustradas o lado esquerdo (verde) e direito (azul) da Eq. (6.10). A solução desta equação para um dado ângulo $\theta$ corresponde ponto em que as curvas interceptam. À medida que $\theta$ aumenta, o ponto de intercessão é maior ou menor dependendo se o fator giromagnético é negativo [painéis (a) e (b)] ou positivo [painéis (c) e (d)]. Utilizando a expressão fenomenológica (6.2) para $\delta \varepsilon[22]$ eu reproduzo a curva teórica [Fig. 6.3(b)] do modelo de Jaroszyński et al. [21], que, por sua vez, é consistente com o resultado do cálculo autoconsistente que eu apresento na Fig. 6.11. Efetuando exatamente o mesmo cálculo, mas agora invertendo o sinal do fator giromagnético efetivo, $g^{*} \rightarrow-g^{*}$, o valor do campo crítico passa a aumentar com $\theta$ [Fig. 6.12(d)].

Este resultado para a dependência do pico anômalo com o sinal do fator giromagnético efetivo é singular pois, neste caso, o efeito Zeeman ordinário, frequentemente desprezado face a magnitude das demais contribuições, agora desempenha um papel fundamental, inclusive acarretando um efeito bastante ro- 
(a)

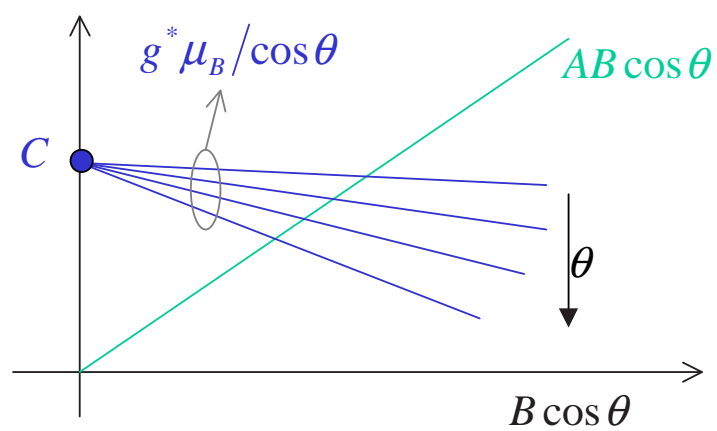

(c)

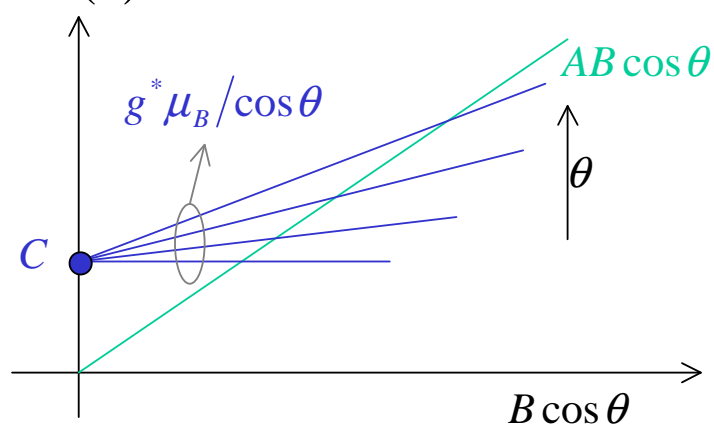

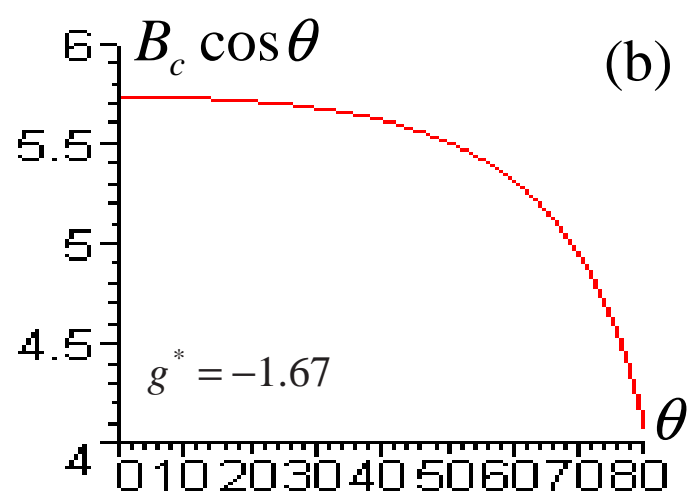

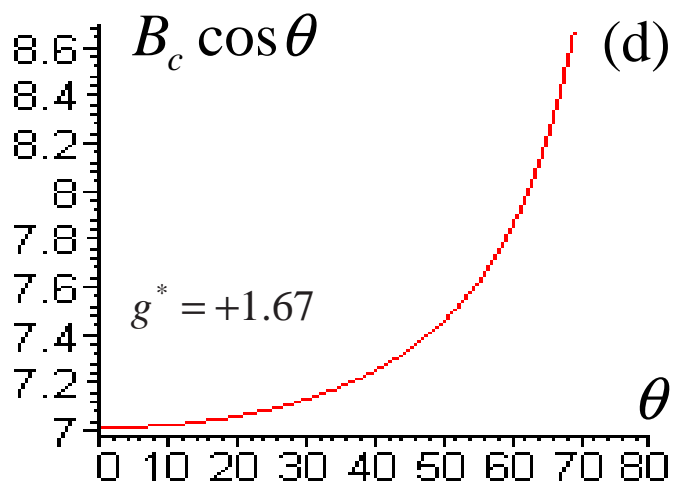

Figura 6.12: Dependência do valor do campo crítico $B_{c}$ em função de $\theta$ com o sinal do fator $g^{*}$. Para cada valor de $\theta$, o campo crítico corresponde ao cruzamento entre as curvas representando os termos $A B \cos \theta$ (verde) e $g^{*} \mu_{B} / \cos \theta$ (azul) nos painéis (a) e (c). Para $g^{*}<0\left(g^{*}>0\right)$ o cruzamento ocorre em $B_{c} \cos \theta$ menor (maior) à medida que o ângulo aumenta. Os painéis $(\mathrm{c})$ e $(\mathrm{d})$ reproduzem a dependência de $B_{c} \cos \theta \operatorname{com} \theta$ utilizando o valor experimental para $\delta \varepsilon$ [Eq. (6.2)], sendo que (c) reproduz a curva teórica da Fig. 6.3(b).

busto. A física por detrás deste efeito é que, quando os níveis de Landau se aproximam, a diferença de energia entre eles passa a ser da ordem do termo Zeeman que, por sua vez, passa a desempenhar um papel muito importante próximo ao cruzamento de níveis.

Histerese Na Ref. [21], a magnitude $\Delta B$ do ciclo de histerese foi definida como a diferença entre os campos $B_{c}$ para $B$ crescendo e diminuindo. Na Fig. 6.13(a) eu apresento os resultados para $\Delta B$ em função da temperatura, e mostro que, de maneira similar aos dados experimentais da Fig. 6.5(a), $\Delta B$ diminui com a temperatura. $\Delta B$ se anula em $T \simeq 2.1 \mathrm{~K}$, indicando que a temperatura crítica da simulação é maior que aquela identificada para o sistema real, $T_{c}=1.3 \mathrm{~K}$. De fato, isto é razoável, uma vez que o sistema real deve conter mais mecanismos que "atrapalham" o ordenamento ferromagnético que o modelo teórico aqui utilizado, resultando então numa temperatura crítica menor [154].

A altura do pico anômalo também varia com a temperatura, Fig. 6.13(b). 

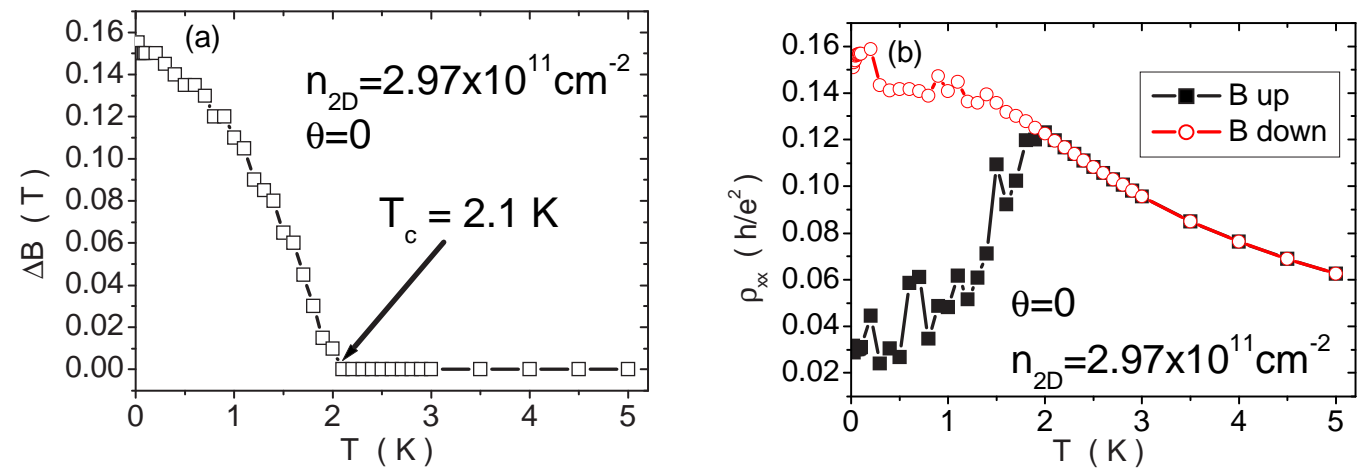

Figura 6.13: Análise do ciclo de histerese em função da temperatura. A magnitude do ciclo de histerese do pico anômalo diminui com a temperatura (a), anulando-se na temperatura identificada como a temperatura crítica para o ferromagnetismo de efeito Hall deste sistema. A altura do pico anômalo também varia com a temperatura (b), sendo que as curvas para $B$ aumentando e diminuindo têm comportamentos diferentes. Para $B$ diminuindo, o máximo de $\rho_{x x}$ diminui com $T$. Já para $B$ aumentando, a altura do pico anômalo aumenta e depois diminui, sendo que o máximo ocorre para para $T=2.1 \mathrm{~K}$, justamente quando as curvas se encontram. As oscilações na curva ocorrem devido a imprecisão numérica.

Entretanto, aqui o comportamento mostrou-se diferente para quando o campo magnético aumenta e diminui. Para $B$ crescendo, a altura do pico anômalo aumenta, ${ }^{15}$ atinge o máximo em $T=2.1 \mathrm{~K}$, e depois volta a diminuir. Já para o caso de $B$ diminuindo, o pico anômalo apenas diminui de amplitude, igualando-se ao caso de $B$ crescendo em $T=2.1 \mathrm{~K}$. Aqui, novamente, eu lembro que as curvas experimentais de $\rho_{x x}$ foram modificadas para excluir a contribuição de SdH usual e, em princípio, mostrar apenas a contribuição intrínseca do pico anômalo. Assim, uma comparação direta da simulação com os dados experimentais deve ser feita com cautela.

De qualquer maneira, o resultado da Fig. 6.13(b) reflete o comportamento diferenciado da estrutura eletrônica e da posição do potencial químico com relação aos estados estendidos, para o campo $B$ aumentando e diminuindo. Como comentado anteriormente, o pico anômalo para $B$ crescendo é pequeno nestas simulações pois somente uma pequena parte da DOS estendida passa pelo potencial químico antes de ocorrer a transição para o outro estado de QHF - ou

\footnotetext{
${ }^{15}$ Acredito que as oscilações na curva de $\rho_{x x}$ da Fig. 6.13 (b) para $T<2 \mathrm{~K}$ se devem a imprecisões numéricas. Como o pico anômalo é muito pequeno e estreito para o caso de $B$ crescendo, pode ser que fosse necessário usar um passo menor para a variação do campo magnético e, assim, registrar efetivamente o valor máximo deste pico na simulação. O passo utilizado foi $0.005 \mathrm{~T}$. Numa versão anterior, com o passo de $0.01 \mathrm{~T}$, estas oscilações foram mais acentuadas e também apareceram oscilações na Fig. 6.13(a), que foram eliminadas ao mudar o passo para $0.005 \mathrm{~T}$.
} 

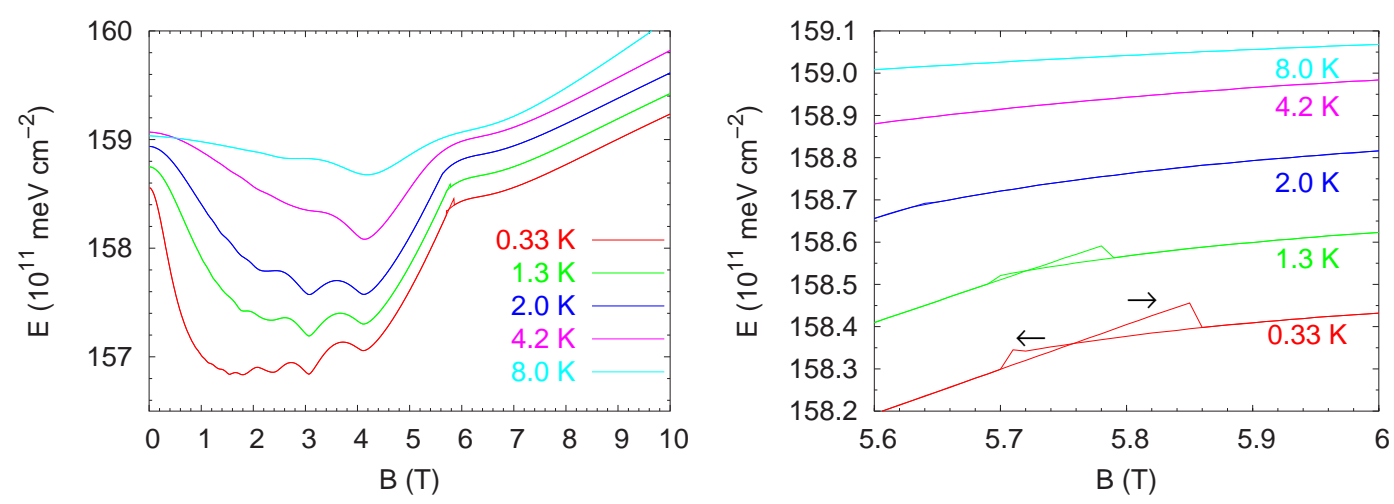

Figura 6.14: Energia total em função do campo magnético para várias temperaturas. (a) Para baixas temperaturas predomina a contribuição da interação de troca s-d. Quando esta satura, a energia orbital dos elétrons confere o carácter oscilatório à medida que diminui o número de níveis de Landau ocupados. Em $B_{c} \simeq 5.7$ T os elétrons passam do nível $|1, \downarrow\rangle$ para $|0, \uparrow\rangle$ ( $B$ crescendo) e por isso a inclinação da curva da energia diminui. Para $T<T_{c}$, a mudança de inclinação é descontínua, indicando uma transição de fase. O painel (b) é uma ampliação da região em torno de $B_{c}$ e as setas indicam os ramos em que $B$ aumenta e diminui. Note que, dentro do ciclo de histerese e para um mesmo valor de campo $B$, os ramos têm energias diferentes.

seja, o pico não chega a atingir o máximo que existiria se não ocorresse a transição. Por outro lado, quando $B$ decresce na simulação, boa parte dos estados estendidos é percorrida pelo potencial químico e, consequentemente, o pico de resistividade correspondente chega a atingir seu máximo antes da transição - o papel da temperatura aqui seria, então, similar ao que ocorre com um pico de SdH usual, ou seja, apenas diminui com $T$. Já para $T>2.1 \mathrm{~K}$ as curvas se sobrepõe, o que era de se esperar, uma vez que o sistema deixa de ser ferromagnético e os casos de $B$ crescendo e diminuindo são equivalentes.

Energia total A energia total do 2DEG é calculada segundo a teoria do funcional da densidade, Eq. (5.9),

$$
\begin{aligned}
E\left[n_{\uparrow}, n_{\downarrow}\right]=\int \varepsilon g(\varepsilon) f(\varepsilon) \mathrm{d} & \varepsilon-\frac{1}{2} \int v_{h}(z) n(z) \mathrm{d} z \\
& -\sum_{\sigma_{z}} \int v_{x c}^{\sigma_{z}}(z) n_{\sigma_{z}}(z) \mathrm{d} z+E_{x c}\left[n_{\uparrow}, n_{\downarrow}\right]-T S_{s}\left[n_{\uparrow}, n_{\downarrow}\right]
\end{aligned}
$$

A Fig. 6.14 apresenta os resultados para a energia total em função do campo magnético para diferentes temperaturas. Novamente, como nos casos apresentados no Cap. 5, o gráfico da energia total em função de $B$ reflete claramente as duas principais contribuições para a energia que dependem explicitamente de $B$. Para baixos campos, prevalece a contribuição da energia de troca s-d que, com o 


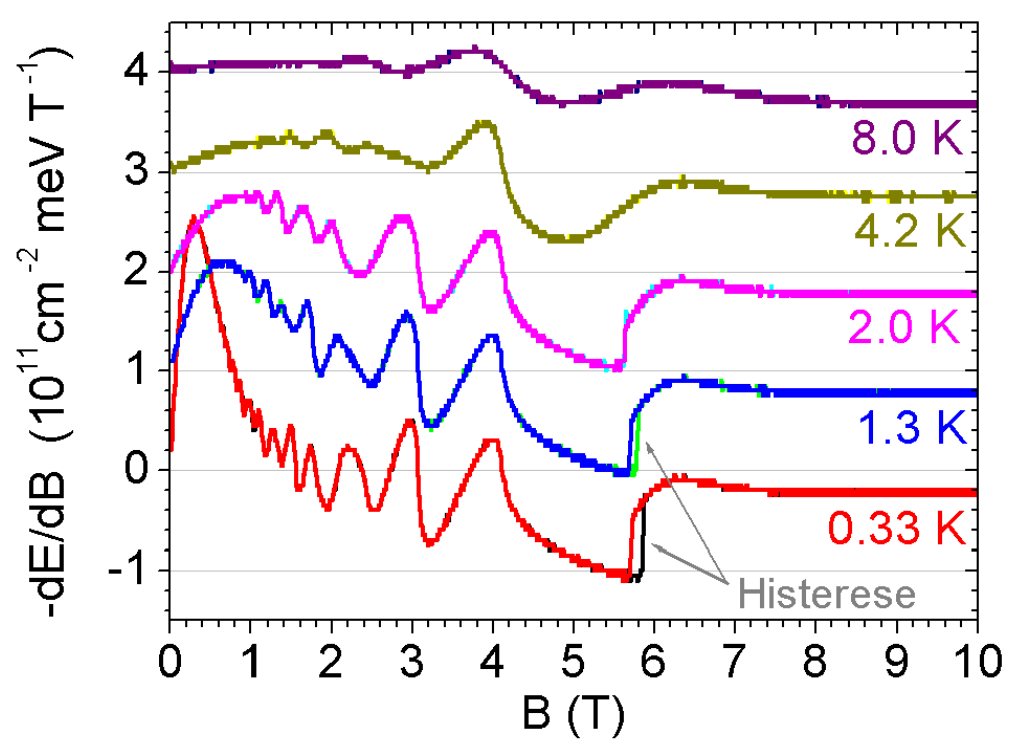

Figura 6.15: Gráfico com a primeira derivada (total) da energia total com relação ao campo magnético. $\mathrm{Em} B \simeq 5.7 \mathrm{~T}$ ocorre tanto a histerese quanto uma variação descontínua da derivada para $T=0.33 \mathrm{~K}$ e $1.3 \mathrm{~K}$. Note que, para $T=2.0 \mathrm{~K}$, não há histerese perceptível, mas a curva é descontínua, em acordo com o resultado da Fig. 6.13(a) que sugere $T_{c} \sim 2.1 \mathrm{~K}$ nas simulações teóricas. As curvas estão deslocadas para facilitar a visualização.

desdobramento de spin gigante, faz com que o 2DEG fique com mais elétrons com spin para baixo (menor energia). Quando esta contribuição satura, em $B \sim 2 \mathrm{~T}$, as oscilações devido a energia de cíclotron predominam.

Em $B_{c} \simeq 5.7 \mathrm{~T}$ ocorre o cruzamento de níveis de Landau, o que acarreta uma mudança na inclinação da curva de $E \times B$. Isto ocorre pois a energia é proporcional ao índice do nível de Landau e, em $B_{c}$, os elétrons no nível $|1, \downarrow\rangle$ passam para o nível $|0, \uparrow\rangle$ quando o campo $B$ aumenta, e vice-versa para $B$ diminuindo. A Fig. 6.14(b), uma ampliação da região em torno de $B_{c}$, mostra não apenas o ciclo de histerese, mas também uma variação descontínua na inclinação das curvas de energia para $T=0.33 \mathrm{~K}$ e $T=1.3 \mathrm{~K}$. A curva para $T=2.0 \mathrm{~K}$ também apresenta esta descontinuidade na inclinação, mas neste gráfico ela é quase imperceptível (este efeito pode ser melhor observado na Fig. 6.15 para a derivada primeira da energia com relação a $B$ ).

Como mencionado no capítulo anterior, neste trabalho eu não efetuei o cálculo da derivada parcial da energia com relação ao campo magnético. Este cálculo forneceria a magnetização do sistema [126]. Em contrapartida, na Fig. 6.15 eu apresento a primeira derivada da energia total com relação ao campo magnético, $-\mathrm{d} E / \mathrm{d} B$, similar ao que foi feito na Sec. 5.3 do capítulo anterior. Novamente, apesar desta grandeza não ser a magnetização do sistema, para $B>2 \mathrm{~T}$ 
as oscilações devem ser similares às oscilações da magnetização do efeito de Haas - van Alphen, uma vez que a dependência de $E\left[n_{\uparrow}, n_{\downarrow}\right]$ com o campo $B$ tem uma forte contribuição da energia orbital.

Da mesma forma que as demais grandezas, a derivada $-\mathrm{d} E / \mathrm{d} B$ também apresenta um ciclo de histerese próximo ao campo crítico $B_{c} \simeq 5.7 \mathrm{~T}$. Para as temperaturas $T=0.33 \mathrm{~K}, 1.3 \mathrm{~K}$ e $2.0 \mathrm{~K}$, as curvas da Fig. 6.15 sofrem uma mudança abrupta (descontínua), enquanto para $T=4.2 \mathrm{~K}$ e $8.0 \mathrm{~K}$, as curvas são suaves (e contínuas). Isto indica que, no campo crítico, o 2DEG sofre uma transição de um estado ferromagnético para outro. Este ferromagnetismo itinerante existe até temperaturas de $T \sim 2.1 \mathrm{~K}$, como também sugere o gráfico para a amplitude da histerese da Fig. 6.13(a).

\subsection{Discussões}

No capítulo anterior eu mostrei que os modelos utilizados neste trabalho para o cálculo da estrutura eletrônica e do magnetotransporte de 2DEGs confinados em DMHs reproduzem satisfatoriamente resultados experimentais para poços quânticos de (Zn,Cd,Mn)Se dopados por modulação [37, 38]. Neste capítulo eu utilizei os mesmos modelos para investigar o magnetotransporte de um 2DEG confinado num sistema de $(\mathrm{Cd}, \mathrm{Mn}) \mathrm{Te}$ [21]. Ao reproduzir satisfatoriamente os resultados experimentais para a resistividade em função do campo magnético, o aparecimento de um pico anômalo em $\rho_{x x}$, e as dependências com $T$ e $\theta$, aqui eu novamente mostrei a adequação do modelo para este tipo de cálculo, reforçando os resultados do capítulo anterior.

Adicionalmente, neste capítulo eu mostrei que, efetuando um cálculo autoconsistente usual da DFT/LSDA, na aproximação da massa efetiva, é possível reproduzir o surgimento do efeito de ferromagnetismo de efeito Hall quântico em 2DEGs confinados em poços quânticos magnéticos. O principal ingrediente do modelo responsável por este resultado é a contribuição de troca-correlação. Quando a polarização $\zeta$ do 2DEG é negativa, XC diminui mais a energia dos elétrons com spin $|\downarrow\rangle$ que aqueles orientados com spin $|\uparrow\rangle$ (cf. Fig. 4.3, pág. 4.3). Esta assimetria, que ocorre quando $\zeta \neq 0$, proporciona uma rigidez magnética [112] no sistema que, em última análise, é responsável pela estabilidade do estado ferromagnético. Assim, quando o campo magnético é variado quando $T<T_{c}$, ocorre uma transição entre dois estados ferromagnéticos em $B=B_{c}$. Esta transição se reflete nas descontinuidades presentes nas curvas de energia dos LLs [Fig. 6.7(a)], de densidade [Fig. 6.7(b)], de energia total [Fig. 6.14(a)] e sua derivada [Fig. 6.14(b)].

Já o surgimento da histerese magnética está associado à existência de 
estados metaestáveis, com energias relativamente próximas ao do estado fundamental do sistema, quando o campo magnético $B$ está próximo do campo crítico $B_{c}$. A Fig. 6.14(a) mostra que, para um mesmo conjunto de parâmetros externos $(B, T, \theta, n)$, o sistema tem energias totais diferentes quando o campo magnético aumenta e quando diminui. O passo utilizado na variação do campo $B$ foi pequeno $(0.01 \mathrm{~T})^{16}$ e utilizei resultado do campo anterior como ponto de partida para o cálculo autoconsistente a cada campo magnético. Assim, posso dizer que esta variação de $B$ foi adiabática, i.e., sem perturbar muito o procedimento autoconsistente de resolução das equações de Kohn-Sham. ${ }^{17}$

Com o procedimento mencionado no parágrafo anterior, o sistema Kohn-Sham retornou soluções que não correspondiam ao estado de mais baixa energia, como ilustra a Fig. 6.14. Como explicado na Sec. 4.2, pág. 89, isto é uma consequência do teorema de Perdew e Levy [115], que diz que os extremos do funcional da energia do sistema são estados estacionários, sendo o estado com a menor energia o estado fundamental, e os demais estados excitados. Como o sistema Kohn-Sham resulta justamente da minimização do funcional da energia, as suas soluções são extremos deste funcional. Desta forma, o resultado que eu obtive mostra que (i) o sistema tem estados disponíveis no espaço de fase próximo de $B_{c}$, e que (ii) dependendo da história da amostra (neste caso, se $B$ aumenta ou diminui), o sistema pode se acomodar em um estado ou outro. Desta forma surge a histerese, que aparece na estrutura eletrônica e, consequentemente, em todas as outras grandezas calculadas.

Note que, apesar de designar estes estados de energia mais alta por metaestáveis, no meu cálculo eles são estados estacionários, soluções das equações de Kohn-Sham. No meu modelo eu não incluo nenhum ingrediente adicional que deixe o sistema relaxar para o estado de mais baixa energia. Já nos sistemas reais estes mecanismos existem. Mesmo no ferromagnetismo usual, os ciclos de histerese ocorrem porque as medidas são efetuadas numa escala de tempo muito menor que o tempo de relaxação. De fato, em um trabalho posterior, o mesmo grupo experimental que efetuou as medidas de magnetotransporte que eu analiso neste capítulo mostrou medidas de relaxação do pico anômalo de resistividade em função do tempo [36]. Estas medidas não apresentam resultados para os ciclos de histerese, mas mostram que os efeitos de relaxação observados no pico de resistividade ocorrem apenas quando $T<T_{c}{ }^{18}$

\footnotetext{
${ }^{16}$ Passos de até $0.1 \mathrm{~T}$ também foram utilizados e os resultados também apresentaram histerese. Passos maiores não são interessantes pois a histerese se dá numa escala de décimos de Tesla, e não seria detectada.

${ }^{17}$ Ao final de cada ciclo autoconsistente a mistura entre o potencial autoconsistente de iterações diferentes é interrompida e outras 10 iterações são calculadas. Assim eu testo se o cálculo convergiu para uma solução estável.

${ }^{18}$ Cooper et al. [34] também interpretou seus resultados para histerese e relaxação presentes
} 
É importante ressaltar que este modelo não recorreu a argumentos sobre a formação de domínios e canais de condução nas barreiras entre domínios para explicar tanto a histerese, quanto o pico de resistividade anômalo. Para reproduzir os resultados experimentais de magnetotransporte foi necessário apenas simular os estados localizados e estendidos dos níveis de Landau, e a passagem do nível de Fermi por estes estados (como na explicação para o efeito Hall quântico inteiro [166]). Assim, mesmo com as evidências de que ocorre uma transição entre estados de QHF, de uma configuração com elétrons $|\uparrow\rangle$ para elétrons $|\downarrow\rangle$, e vice-versa, mais a histerese no pico de resistividade anômalo, estas não são evidências diretas da formação de domínios. Neste sentido, a presente formulação é uma proposta alternativa para explicar os resultados experimentais de magnetotransporte no regime de ferromagnetismo de efeito Hall. Entretanto, isto não significa que domínios de fato não ocorram, e que eles também não influenciem nas propriedades de magnetotransporte.

Revisando os resultados de Knobel et al. [37] Para finalizar este capítulo, eu observo que Jaroszyński et al. [21] citam o experimento da Ref. [37], analisado no capítulo anterior, como um exemplo em que ocorre o cruzamento de LLs mas que não há o efeito QHF. De fato, Knobel et al. [37] fazem experimentos em temperaturas tão baixas quanto $0.36 \mathrm{~K}$, observam o aparecimento de um pico anômalo em $\rho_{x x}$, mas não há nenhuma histerese ou outro sinal de QHF. Isto é uma confirmação do fato que o pico anômalo ocorre devido ao cruzamento de LLs, ou seja, não é necessário que ocorra o efeito de QHF para o seu aparecimento.

A questão que se coloca é, uma vez que os regimes dos dois experimentos são tão semelhantes, com o 2DEG parcialmente polarizado e com o cruzamento de LLs de spins opostos, porque não foi observado QHF nos resultados da Ref. [37] semelhantes àqueles apresentados na Ref. [21] ${ }^{19}$ Eu considero dois pontos que podem explicar a diferença nos resultados:

1. O efeito de QHF é mais acentuado quando o fator de preenchimento é próximo de um valor inteiro [155]. Quando isto ocorre, a diminuição da energia dos elétrons majoritários devido a troca-correlação é mais acentuada. O sistema estudado por Jaroszyński et al. [21] satisfaz esta condição $(\nu \simeq 2)$. Já no sistema de Knobel et al. [37], o pico anômalo surge em um valor de campo magnético tal que $\nu \simeq 2.6$, fora da condição óptima para a ocorrência do QHF.

em curvas de $\rho_{x x}$ em termos de estados metaestáveis.

${ }^{19}$ Ao realizar os experimentos divulgados na Ref. [37], os autores estavam também procurando algum sinal de QHF, e esperavam encontrar uma histerese no pico anômalo, o que não aconteceu [R. Knobel, comunicação privada]. 
2. Somando-se ao item anterior, a amostra de Knobel et al. [37] têm mobilidade menor que a de Jaroszyński et al. [21]. Isto significa que há mais espalhamento que, por sua vez, pode suprimir a estabilidade de fases ferromagnéticas [163]. 


\section{Capítulo 7}

\section{Conclusões}

Nesta tese eu investiguei a física dependente de spin presente em poços quânticos digitais magnéticos do grupo II-VI. O contexto da abordagem teórica foi a versão dependente de spin da teoria do funcional da densidade (DFT) na aproximação da densidade de spin local (LSDA). Eu resolvi as equações autoconsistentes de Kohn-Sham para a função envelope na aproximação da massa efetiva. Em seguida eu calculei diversas propriedades experimentalmente relevantes.

Eu separei o trabalho em duas partes. Na primeira eu estudei os efeitos de aglomeração e diluição das impurezas de Mn. Analisei a influência destes efeitos na estrutura eletrônica do sistema e, consequentemente, nas propriedades magnetoópticas experimentalmente investigadas. Eu defini perfis contínuos para modelar as concentrações nominais e efetivas. Estes perfis levam em conta a diluição das impurezas, seja por segregação ou por interdifusão. O perfil de concentração nominal foi utilizado para determinar a modulação estrutural dos offsets na banda de condução devido a presença dos átomos de Mn na liga semicondutora. Já o perfil de concentração efetiva considera a aglomeração antiferromagnética entre os íons de Mn, e foi utilizada para modelar o potencial dependente de spin que os portadores sentem ao longo da amostra devido a interação de troca sp-d.

Usando os modelos para aglomeração e diluição, eu simulei o desdobramento de spin gigante em DMHs investigadas por magnetofotoluminescência $[4,7]$. Esta parte do trabalho foi uma continuação do trabalho da Ref. [39], no qual foi utilizado um perfil quadrado (sem diluição) mais o modelo de singletos para a aglomeração antiferromagnética; o trabalho mostrou a importância da aglomeração, mas o acordo com os dados numéricos foi qualitativo. Agora, com a inclusão da diluição mais o modelo de estatística de aglomerados com singletos e tripletos, foi possível obter um acordo quantitativo com os resultados experimentais.

Nesta parte do trabalho eu também calculei tempos de espalhamento de spin nas mesmas DMHs. Este cálculo é mais ilustrativo uma vez que o espa- 
lhamento induzido pela interação de troca $s-d$ provavelmente não é o mecanismo dominante nestes sistemas. O aspecto importante neste cálculo incluindo o efeito de diluição foi mostrar que o efeito dos fatores de Fermi sobre $\tau_{s f}$ continua sendo aumentar o desdobramento entre os ramos, um para transições de spin para cima para spin para baixo, e o outro o contrário. Isto reforçou o resultado do trabalho anterior [39]. Adicionalmente, foi possível mostrar que a inclusão de diluição fez com que os tempos de espalhamento calculados teoricamente ficassem mais próximos entre si para diferentes configurações, quando comparado com o caso sem diluição, reproduzindo esta característica presente nos dados experimentais.

Uma vez estabelecido que os modelos contínuos para os perfis de concentração de Mn fornecem bons resultados, passei então a estudar o física do gás de elétrons bidimensional que se forma quando os DMQWs são dopados, Caps. 5 e 6. Os perfis modelo continuaram a ser usados, não mais como o objeto do estudo, mas como parte da formulação. Nesta segunda parte do trabalho, eu estudei os resultados de dois conjuntos de dados experimentais. Apesar dos sistemas serem baseados em diferentes materiais, ZnSe nas Refs. [37, 38] e CdTe na Ref. [21], ambos são parecidos em diversos aspectos. Os sistemas em questão são poços quânticos digitais magnéticos dopados por modulação que são submetidos a medidas de magnetotransporte no regime do efeito Hall quântico inteiro. Ambos experimentos observaram o aparecimento de um pico anômalo nas medidas do efeito Shubnikov-de Haas. Entretanto, apenas a Ref. [21] detectou ciclos de histerese indicando o regime de ferromagnetismo de efeito Hall quântico.

Usando o modelo apresentado no Cap. 4 mais o modelo para descrever o potencial de troca $s-d$ formulado nos Caps. 2 e 3, eu calculei a estrutura eletrônica e o magnetotransporte para os dois sistemas experimentais mencionados. Para os dois casos, os meus resultados reproduziram as curvas de magnetotransporte em função do campo magnético razoavelmente bem, bem como a dependência com a temperatura e o ângulo do campo $B$ com relação à direção de crescimento do amostra. Picos anômalos nas curvas de resistividade $\rho_{x x}$ surgiram naturalmente, ficando evidente que a origem destes é o cruzamento entre níveis de Landau com spins opostos próximos ao nível de Fermi.

Um primeiro aspecto que ficou evidente nestes cálculos, foi a importância da contribuição dos efeitos de muitos corpos (troca e correlação) para o desdobramento de spin presente na estrutura eletrônica destes sistemas dopados. As estimativas feitas pelos grupos experimentais, considerando que apenas a interação de troca $s-d$ contribui para o desdobramento de spin, resultam em concentrações de Mn maiores que os valores nominais incorporados nas amostras. A simulação que fiz na aproximação Hartree (Cap. 5) também precisa de valores maiores de Mn, confirmando esta interpretação. Por outro lado, ao incluir 
troca-correlação, a contribuição da interação de troca s-d para o desdobramento de spin precisa ser menor para manter o mesmo desdobramento de spin final, e portanto, os valores para as concentrações de Mn também são menores, e mais realistas.

O ponto mais interessante nestas simulações foi o aparecimento do efeito de ferromagnetismo de efeito Hall quântico no 2DEG confinado na DMH de (Cd,Mn)Te, reproduzindo os ciclos de histerese e a temperatura crítica presentes nos resultados experimentais da Ref. [21]. O ingrediente da formulação que permitiu o aparecimento destes efeitos foi o termo de troca e correlação dependente de spin. Troca e correlação no gás de elétrons atenua a contribuição de Hartree, sendo este efeito mais acentuado quando o sistema está polarizado, ou seja, com todos os spins com a mesma orientação. Este efeito é capaz de estabilizar o estado ferromagnético, que persiste enquanto a temperatura do sistema é menor que uma certa temperatura crítica. O cálculo que eu efetuei mostra que, no espaço de configurações do sistema, existem estados metaestáveis relativamente próximos quando níveis de Landau de spins opostos se aproximam. Dependendo se o campo magnético está aumentando ou diminuindo, o sistema pode ficar no estado de menor energia ou em um destes estados excitados, resultando então um ciclo de histerese.

É então importante frisar que o modelo formulado neste trabalho é uma explicação alternativa para os efeitos de ferromagnetismo de efeito Hall quânticos observados em experimentos de magnetotransporte. Isto porque, a visão mais divulgada na literatura envolve a formação de domínios no 2DEG, e que os picos anômalos de resistividade resultam da condução nas barreiras entre estes domínios. Note, porém, que o fato dos resultados experimentais terem sido reproduzidos sem considerar a formação de domínios não exclui existência deles, mas indica que eles não são essenciais para a ocorrência do efeito. Adicionalmente, este trabalho é o primeiro que, de fato, efetua um cálculo de magnetotransporte no regime de ferromagnetismo de efeito Hall quântico, e que reproduz alguns dos resultados experimentais existentes na literatura.

Aqui eu também explico porque não foi observado o efeito de QHF no sistema baseado em (Zn,Mn)Se das Refs. [37, 142]. Neste sistema, o fator de preenchimento do 2DEG próximo ao campo magnético do pico anômalo não é inteiro (e nem próximo), não satisfazendo assim a condição que acentua a interação de troca-correlação, que, por sua vez, estabiliza o estado ferromagnético [155]. Além disso, a amostra apresenta mais desordem, indicado pela mobilidade menor, sendo este um outro fator que atrapalha a formação do estado ferromagnético [163].

Resumindo os principais resultados deste trabalho, primeiro eu mos- 
trei que, ao modelar a distribuição das impurezas magnéticas em DMHs usando perfis de concentração nominal e efetivos, considerando conjuntamente os efeitos de diluição e de aglomeração antiferromagnética, obtenho uma boa descrição do potencial dependente de spin presente nestes sistemas, que por sua vez, pode ser utilizado no cálculo da estrutura eletrônica. O segundo resultado é sobre a importância da interação de muitos corpos nestes sistemas bidimensionais magnéticos: por um lado troca e correlação têm uma contribuição considerável no desdobramento de spin destes sistemas; por outro lado, como os 2DEGs confinados nestes sistemas magnéticos são quase que completamente polarizados, a dependência de troca-correlação com a polarização é bem mais acentuada que em sistemas nãomagnéticos. Adicionalmente, ao considerar os efeitos de troca e correlação fui capaz de reproduzir o aparecimento do efeito de ferromagnetismo de efeito Hall quântico, simulando ciclos de histerese, transição de fase e temperatura crítica.

Este trabalho fornece uma prescrição teórica relativamente simples para estudar a física dependente de spin de heteroestruturas magnéticas. Ela é baseada no formalismo de Kohn-Sham da teoria do funcional da densidade, no qual o problema de muitos corpos é mapeados em um sistema efetivo de partícula simples. Os resultados tiveram um bom acordo com vários dados experimentais, sugerindo que as contribuições mais relevantes para o sistema foram adequadamente consideradas. Naturalmente, muitas melhorias podem ser acrescentadas ao presente modelo, e novos tópicos podem ser investigados à partir do ferramental que foi desenvolvido para este trabalho.

Em especial, aqui eu menciono alguns projetos que já estão em andamento. Com relação ao procedimento numérico, estão sendo desenvolvidas rotinas para resolver as equações de Kohn-Sham usando uma discretização não-uniforme, ${ }^{1}$ o que permitirá efetuar os mesmos cálculos em menos tempo e com maior precisão. Outro projeto em andamento é um estudo comparativo entre diversos funcionais e parametrizações para a energia e o potencial de troca-correlação, nas aproximações LSDA e GGA. ${ }^{2}$ Este estudo investiga a adequação destes funcionais quando se utiliza a DFT no cálculo da estrutura eletrônica de heteroestruturas semicondutoras magnéticas. Outro projeto em andamento é o estudo de heteroestruturas rasas, ou seja, que o potencial de confinamento é pequeno o suficiente tal que apenas parte do elétrons da região dopada é capturada pela região de confinamento (cf. a nota de rodapé 2, pág. 111). Como já mencionado, estou também trabalhando no cálculo da magnetização eletrônica do sistema. Esta é uma grandeza relevante pois é usada para investigar a densidade de estados do sistema eletrônico. Em particular, quando ocorre o efeito de QHF, esta grandeza

\footnotetext{
${ }^{1}$ Colaboração com o estudante de iniciação científica Gerson Ferreira Junior.

${ }^{2}$ Colaboração com o Prof. Dr. Klaus W. Capelle.
} 
é muito importante pois pode trazer informações sobre a natureza das transições de fase que ocorrem nestes sistemas. Também pretende-se calcular a susceptibilidade magnética e calor específico.

Além destes projetos em andamento, várias outras possibilidades podem ser exploradas. Nos últimos anos foram divulgados vários experimentos mostrando o aparecimento do ferromagnetismo de efeito Hall quântico em 2DEGs confinados em vários tipos de heteroestruturas [21-35]. O modelo teórico desenvolvido neste trabalho permite investigar muitos destes sistemas. Além do mais, como o modelo aqui proposto é relativamente simples, ele pode ser utilizado para investigar o espaço de parâmetros de heteroestruturas ainda a serem crescidas. Muitos dos experimentos divulgados na literatura são no regime do efeito Hall quântico fracionário, muitos deles com medidas de magnetotransporte que exibem picos anômalos e histerese. Assim, um projeto interessante será utilizar a formulação de férmions compostos para mapear o efeito fracionário no efeito inteiro $[169,170]$ e, então, possivelmente aproveitar a formulação deste projeto para investigar o magnetotransporte destes sistemas fracionários.

Aqui eu aponto a necessidade de se efetuar medidas sistemáticas investigando a física do Mn em arranjos bidimensionais de heteroestruturas digitais, o que pode ser feito usando técnicas de espectroscopia magnetoóptica tradicionais ou a técnica de microscopia de tunelamento de seção transversal (X-STM) [84-88]. Medidas deste tipo ajudariam aprofundar o entendimento sobre os efeitos de aglomeração antiferromagnética e de diluição, com importante impacto na engenharia de sistemas magnéticos nanoestruturados. Nos parágrafos anteriores eu cito várias possibilidades de trabalhos teóricos, com destaque especial para aqueles envolvendo a física de 2DEGs no regime de ferromagnetismo de efeito Hall. Trabalhos experimentais neste sistemas, mas envolvendo outras grandezas, como magnetização, susceptibilidade magnética, e/ou calor específico, provavelmente fornecerão valiosas informações sobre a natureza das transições de fase que ocorrem nestes sistemas. Assim, com este trabalho eu espero estimular diversas linhas de investigação, sejam elas teóricas e/ou experimentais. 


\section{Apêndice A}

\section{Tempo de espalhamento de spin em DMHs}

\section{A.1 Introdução}

Aqui eu reproduzo o modelo de espalhamento de spin induzido pela interação de troca $s$ - $d$ apresentado nas Refs. $[39,83]$ e utilizado no Cap. 3 desta tese. Na Sec. 3.4 eu inicio a análise à partir das taxas de espalhamento deduzidas com detalhes na Ref. [83]; como esta referência é uma tese, cujo texto é de acesso mais difícil, este apêndice se faz necessário para tornar o texto da tese autosuficiente. No cálculo das taxas de espalhamento de spin que segue eu sigo os passos da Ref. [83], mas também generalizo o cálculo para um perfil arbitrário (na direção de crescimento) de concentração de impurezas magnéticas (as Refs. [39, 83] apresentam o caso particular de barreiras ideais quadradas).

A interação entre os elétrons itinerantes da banda de condução com os elétrons localizados das impurezas magnéticas é chamada de interação de troca $s$-d, e pode ser descrita por um hamiltoniano do tipo Kondo (cf. Sec. 1.4.3)

$$
H_{s-d}=-\sum_{R_{j}} J\left(r-R_{j}\right) S_{R_{j}} \cdot \sigma
$$

Excetuando a Sec. 3.4, no restante desta tese eu utilizei a aproximação de campo médio (MF) para interação de troca $s$ - $d$, cujo hamiltoniano é

$$
H_{s-d}^{M F}=-\left\langle S_{z}\right\rangle \sigma_{z} \sum_{R_{j}} J\left(r-R_{j}\right)
$$

Este hamiltoniano de campo médio é completamente diagonal e não acarreta processos de espalhamento de spin. Entretanto, as flutuações em relação a situação média, ou seja, os termos de (A.1) que foram desprezados, podem, em princípio, 
ser responsáveis por spin-flip. As flutuações da situação de campo médio podem ser escritas como

$$
\begin{aligned}
\Delta H_{s-d} & =H_{s-d}-H_{s-d}^{M F} \\
& =-\sum_{R_{j}} J\left(r-R_{j}\right)\left[\left(S_{z, R_{j}}-\left\langle S_{z}\right\rangle\right) \sigma_{z}+\frac{1}{2}\left(S_{R_{j}}^{+} \sigma^{-}+S_{R_{j}}^{-} \sigma^{+}\right)\right] .
\end{aligned}
$$

Os termos fora da diagonal de (A.3),

$$
H_{s f}=H^{\uparrow \downarrow}+H^{\downarrow \uparrow}=-\frac{1}{2} \sum_{R_{j}} J\left(r-R_{j}\right)\left(S_{R_{j}}^{+} \sigma^{-}+S_{R_{j}}^{-} \sigma^{+}\right),
$$

induzem o espalhamento de spin.

Para calcular o tempo de espalhamento de um estado eletrônico $\underline{i n i c i a l ~ c o m ~ s p i n ~} \sigma_{z}$ para um estado $\underline{f}$ inal com spin $\bar{\sigma}_{z}$ utiliza-se então a regra de ouro de Fermi,

$$
\frac{1}{\tau_{s f}^{\sigma \bar{\sigma}}}=\frac{2 \pi}{\hbar} \sum_{k_{f}}\left\langle\left|\left\langle f\left|H_{s f}\right| i\right\rangle\right|^{2}\right\rangle_{s p i n} \delta\left(E_{f}^{\bar{\sigma}}-E_{i}^{\sigma}\right),
$$

sendo $H_{s-f}$ o hamiltoniano de spin-flip (A.4), e $E_{i}^{\sigma}$ e $E_{f}^{\bar{\sigma}}$ as energias dos estados inicial e final, respectivamente.

\section{A.2 Elemento de matriz do espalhamento de spin}

Para a primeira sub-banda, as transições entre spin $|\sigma\rangle$ e spin $|\bar{\sigma}\rangle$ envolvem os seguintes estados iniciais e finais

$$
\begin{aligned}
|i\rangle & =\frac{\mathrm{e}^{\mathrm{i} k_{i} \cdot r}}{\sqrt{A}} \chi^{\sigma}(z) u_{1,0}(r)|\sigma\rangle\left|S_{z}^{i}\left(R_{1}\right) \cdots S_{z}^{i}\left(R_{j}\right) \cdots S_{z}^{i}\left(R_{N}\right)\right\rangle, \\
|f\rangle & =\frac{\mathrm{e}^{\mathrm{i} k_{f} \cdot r}}{\sqrt{A}} \chi^{\bar{\sigma}}(z) u_{1,0}(r)|\bar{\sigma}\rangle\left|S_{z}^{f}\left(R_{1}\right) \cdots S_{z}^{f}\left(R_{j}\right) \cdots S_{z}^{f}\left(R_{N}\right)\right\rangle
\end{aligned}
$$

nos quais $u_{1,0}(r)$ é a função de Bloch em $\boldsymbol{k}=0$ [42] da primeira sub-banda. Desta forma, e.g., o elemento de matriz para a transição spin $|\uparrow\rangle$ para spin $|\downarrow\rangle$ fica

$$
\begin{aligned}
\left\langle f\left|H^{\uparrow \downarrow}\right| i\right\rangle & =\left\langle f\left|-\frac{1}{2} \sum_{R_{j}} J\left(r-R_{j}\right) S_{R_{j}}^{+} \sigma^{-}\right| i\right\rangle \\
& =-\frac{1}{2} \sum_{R_{j}} \int_{V} \frac{\mathrm{e}^{\mathrm{i}\left(k_{i}-k_{f}\right) \cdot r}}{A} \chi^{\uparrow}(z) \chi^{\downarrow}(z) u_{1,0}^{*}(r) J\left(r-R_{j}\right) u_{1,0}(r) \mathrm{d} r \times\left\langle\downarrow\left|\sigma^{-}\right| \uparrow\right\rangle \\
& \times\left\langle S_{z}^{f}\left(R_{1}\right) \cdots S_{z}^{f}\left(R_{j}\right) \cdots S_{z}^{f}\left(R_{N}\right)\left|S_{R_{j}}^{+}\right| S_{z}^{i}\left(R_{1}\right) \cdots S_{z}^{i}\left(R_{j}\right) \cdots S_{z}^{i}\left(R_{N}\right)\right\rangle(\mathrm{A} .8)
\end{aligned}
$$


Mudando de variáveis $r \rightarrow r^{\prime}+R_{j}$ e usando que $u_{n, 0}\left(r^{\prime}\right)=u_{n, 0}\left(r^{\prime}+R_{j}\right)$, a parte espacial de (A.8) fica

$$
-\frac{1}{2} \sum_{R_{j}} \frac{\mathrm{e}^{\mathrm{i}\left(k_{i}-k_{f}\right) \cdot R_{j}}}{A} \int_{V} \mathrm{e}^{\mathrm{i}\left(k_{i}-k_{f}\right) \cdot r^{\prime}} \chi^{\uparrow}\left(z^{\prime}+Z_{j}\right) \chi^{\downarrow}\left(z^{\prime}+Z_{j}\right) u_{1,0}^{*}\left(r^{\prime}\right) J\left(r^{\prime}\right) u_{1,0}\left(r^{\prime}\right) \mathrm{d} r .
$$

Uma vez que o acoplamento de troca $J(r)$ é de curto alcance, simplifica-se (A.9) fazendo $r^{\prime}=0$ e $z^{\prime}=0$ (interação de contato), resultando em

$$
-\frac{1}{2} \sum_{R_{j}} \frac{\mathrm{e}^{\mathrm{i}\left(k_{i}-k_{f}\right) \cdot R_{j}}}{A} \chi^{\uparrow}\left(Z_{j}\right) \chi^{\downarrow}\left(Z_{j}\right) \int_{V} u_{1,0}^{*}\left(r^{\prime}\right) J\left(r^{\prime}\right) u_{1,0}\left(r^{\prime}\right) \mathrm{d} r .
$$

Usando que

$$
\begin{gathered}
\left\langle\downarrow\left|\sigma^{-}\right| \uparrow\right\rangle=1, \\
\int_{V} u_{1,0}^{*}\left(r^{\prime}\right) J\left(r^{\prime}\right) u_{1,0}\left(r^{\prime}\right) \mathrm{d} r=\alpha,
\end{gathered}
$$

e que o elemento de matriz relativo ao spin do Mn é

$$
\begin{gathered}
\left\langle S_{z}^{f}\left(R_{1}\right) \cdots S_{z}^{f}\left(R_{j}\right) \cdots S_{z}^{f}\left(R_{N}\right)\left|S_{R_{j}}^{+}\right| S_{z}^{i}\left(R_{1}\right) \cdots S_{z}^{i}\left(R_{j}\right) \cdots S_{z}^{i}\left(R_{N}\right)\right\rangle \\
=\left\langle S_{z}^{f}\left(R_{1}\right) \mid S_{z}^{i}\left(R_{1}\right)\right\rangle \times \\
\cdots \times\left\langle S_{z}^{f}\left(R_{j}\right)\left|S_{R_{j}}^{+}\right| S_{z}^{i}\left(R_{j}\right)\right\rangle \times \cdots \\
\times\left\langle S_{z}^{f}\left(R_{N}\right) \mid S_{z}^{i}\left(R_{N}\right)\right\rangle \\
=\sqrt{S(S+1)-S_{z}^{i}\left(R_{j}\right)\left[S_{z}^{i}\left(R_{j}\right)+1\right]} \delta_{S_{z}^{f}\left(R_{j}\right), S_{z}^{i}\left(R_{j}\right)+1} \prod_{l \neq j} \delta_{S_{z}^{f}\left(R_{l}\right), S_{z}^{i}\left(R_{l}\right)},
\end{gathered}
$$

o termo $\left\langle f\left|H^{\uparrow \downarrow}\right| i\right\rangle$ para a transição $\uparrow \downarrow$ fica

$$
\begin{aligned}
& \left\langle f\left|H^{\uparrow \downarrow}\right| i\right\rangle=-\frac{\alpha}{2} \sum_{R_{j}} \frac{\mathrm{e}^{\mathrm{i}\left(k_{i}-k_{f}\right) \cdot R_{j}}}{A} \chi^{\uparrow}\left(Z_{j}\right) \chi^{\downarrow}\left(Z_{j}\right) \\
& \sqrt{S(S+1)-S_{z}^{i}\left(R_{j}\right)\left[S_{z}^{i}\left(R_{j}\right)+1\right]} \delta_{S_{z}^{f}\left(R_{j}\right), S_{z}^{i}\left(R_{j}\right)+1} \prod_{l \neq j} \delta_{S_{z}^{f}\left(R_{l}\right), S_{z}^{i}\left(R_{l}\right)} .
\end{aligned}
$$

O termo relevante na regra de ouro de Fermi é o módulo ao quadrado do elemento de matriz conectando os estados iniciais e finais, a saber,

$$
\begin{aligned}
\left|\left\langle f\left|H^{\uparrow \downarrow}\right| i\right\rangle\right|^{2} & =\frac{1}{4} \frac{1}{A^{2}} \alpha^{2} \sum_{R_{j}}\left|\chi^{\uparrow}\left(Z_{j}\right)\right|^{2}\left|\chi^{\downarrow}\left(Z_{j}\right)\right|^{2} \\
\times & \left\{S(S+1)-S_{z}^{i}\left(R_{j}\right)\left[S_{z}^{i}\left(R_{j}\right)+1\right]\right\} .
\end{aligned}
$$




\section{A.3 Média sobre as impurezas}

Agora é necessário efetuar a média térmica sobre as orientações dos spins das impurezas $\langle\cdots\rangle_{\text {spin }}$ [as coordenadas das impurezas em (A.15) são bem definidas], ou seja,

$$
\begin{aligned}
\left\langle\left|\left\langle f\left|H^{\uparrow \downarrow}\right| i\right\rangle\right|^{2}\right\rangle_{\text {spin }} & =\frac{1}{4} \frac{1}{A^{2}} \alpha^{2} \sum_{R_{j}}\left|\chi^{\uparrow}\left(Z_{j}\right)\right|^{2}\left|\chi^{\downarrow}\left(Z_{j}\right)\right|^{2} \\
& \times\left\langle S(S+1)-S_{z}^{i}\left(R_{j}\right)\left[S_{z}^{i}\left(R_{j}\right)+1\right]\right\rangle_{\text {spin }}
\end{aligned}
$$

Esta média recai sobre o termo contendo o spin do Mn, que fica

$$
\left\langle S(S+1)-S_{z}^{i}\left(R_{j}\right)\left[S_{z}^{i}\left(R_{j}\right)+1\right]\right\rangle_{\text {spin }}=S(S+1)-\left\langle S_{z}^{2}\right\rangle-\left\langle S_{z}\right\rangle
$$

sendo

$$
\left\langle S_{z}^{2}\right\rangle=\frac{\sum_{S_{z}=-S}^{S} S_{z}^{2} \exp \left(-g_{M n} \mu_{B} B S_{z} / k_{B} T\right)}{\sum_{S_{z}=-S}^{S} \exp \left(-g_{M n} \mu_{B} B S_{z} / k_{B} T\right)}
$$

$\mathrm{e}$

$$
\left\langle S_{z}\right\rangle=\frac{\sum_{S_{z}=-S}^{S} S_{z} \exp \left(-g_{M n} \mu_{B} B S_{z} / k_{B} T\right)}{\sum_{S_{z}=-S}^{S} \exp \left(-g_{M n} \mu_{B} B S_{z} / k_{B} T\right)}=-S B_{S}\left(\frac{g_{M n} \mu_{B} S B}{k_{B} T}\right),
$$

$\operatorname{com} B_{s}(x)$ a função de Brillouin

$$
B_{S}(x)=\left(1+\frac{1}{2 S}\right) \operatorname{coth}\left[\left(1+\frac{1}{2 S}\right) x\right]-\frac{1}{2 S} \operatorname{coth}\left(\frac{1}{2 S} x\right) .
$$

A Eq. (A.16) simplifica então para

$$
\left\langle\left|\left\langle f\left|H^{\uparrow \downarrow}\right| i\right\rangle\right|^{2}\right\rangle_{\text {spin }}=\frac{\alpha^{2}}{4 A^{2}}\left\{S(S+1)-\left\langle S_{z}^{2}\right\rangle-\left\langle S_{z}\right\rangle\right\} \sum_{R_{j}}\left|\chi^{\uparrow}\left(Z_{j}\right)\right|^{2}\left|\chi^{\downarrow}\left(Z_{j}\right)\right|^{2}
$$

Para realizar a soma sobre os sítios das impurezas, eu uso o fato que elas estão distribuídas em $N_{b}$ camadas, cada uma contendo $N_{b}^{M n}$ impurezas magnéticas. Em cada plano, o número de íons de Mn é $N_{b}^{M n}$, que pode ser escrito em termos da concentração molar planar e do número total de cations, $N_{b}^{M n}=x_{p} N_{b}^{c}=x_{p} A d / \Omega_{0}$, com $A d$ o volume da camada com largura $d$ e área $A$, e $\Omega_{0}$ o volume da célula primitiva. Resumindo,

$$
\sum_{R_{j}}=\sum_{n=1}^{N_{b}} N_{b}^{M n}=\sum_{n=1}^{N_{b}} x_{p} N_{b}^{c}=\sum_{n=1}^{N_{b}} x_{p} \frac{A d}{\Omega_{0}}=\frac{A}{\Omega_{0}} \sum_{n=1}^{N_{b}} x_{p} d \underset{(2.28)}{\stackrel{(2.27)}{\longrightarrow}} \frac{A}{\Omega_{0}} \int_{L} x(z) \mathrm{d} z .
$$


Na última passagem de (A.22) eu uso a propriedade de normalização (2.27) da função perfil $\wp(z)$ para estender o caso ideal, perfil quadrado com largura $d$, para o caso contínuo com um perfil arbitrário de concentração de Mn. Assim, usando que $N_{0}=\Omega_{0}^{-1}$, a Eq. (A.21) fica

$$
\begin{aligned}
\left\langle\left|\left\langle f\left|H^{\uparrow \downarrow}\right| i\right\rangle\right|^{2}\right\rangle_{\text {spin }}=\frac{\Omega_{0}}{4 A} & \left(N_{0} \alpha\right)^{2}\left\{S(S+1)-\left\langle S_{z}^{2}\right\rangle-\left\langle S_{z}\right\rangle\right\} \\
& \times \int_{L} x(z)\left|\chi^{\uparrow}\left(Z_{j}\right)\right|^{2}\left|\chi^{\downarrow}\left(Z_{j}\right)\right|^{2} \mathrm{~d} z .
\end{aligned}
$$

\section{A.4 Taxa de espalhamento de spin}

Agora eu calculo a taxa de espalhamento de spin, a partir da qual defini-se o tempo característico de spin-flip $\tau_{s f}$. Inicialmente eu não incluo os fatores de Fermi. Em seguida eu considero a ocupação das sub-bandas admitindo que os portadores nas sub-bandas são descritos pela função de Fermi com uma temperatura efetiva $T_{e}$ maior que a temperatura de rede cristalina $T_{L}$ (o processo ocorre numa escala de tempo inferior à necessária para ocorrer a termalização com a rede).

\section{A.4.1 Caso sem estatística de Fermi}

A taxa de espalhamento de spin é dada pela Eq. (A.5). Substituindo (A.23) em (A.5) obtenho

$$
\begin{aligned}
& \frac{1}{\tau_{s f}^{\uparrow \downarrow}}=\frac{2 \pi}{\hbar} \sum_{k_{\|}^{f}} \frac{\Omega_{0}}{4 A}\left(N_{0} \alpha\right)^{2}\{S(S+1)\left.-\left\langle S_{z}^{2}\right\rangle-\left\langle S_{z}\right\rangle\right\} \\
& \times \int_{L} x(z)\left|\chi^{\uparrow}\left(Z_{j}\right)\right|^{2}\left|\chi^{\downarrow}\left(Z_{j}\right)\right|^{2} \mathrm{~d} z \\
& \times \delta\left(E_{1}^{\downarrow}+\frac{\hbar^{2} k_{\|}^{f^{2}}}{2 m}-E_{1}^{\uparrow}-\frac{\hbar^{2} k_{\|}^{i^{2}}}{2 m}\right) .
\end{aligned}
$$

Substituindo $\sum_{k_{\|}^{f}}$ por $A /(2 \pi)^{2} \int_{0}^{2 \pi} \mathrm{d} \theta \int_{0}^{\infty} k_{\|}^{f} \mathrm{~d} k_{\|}^{f}$, e usando que $k_{\|}^{f} \mathrm{~d} k_{\|}^{f}=\frac{1}{2} \mathrm{~d}\left(\frac{\hbar^{2} k_{\|}^{f^{2}}}{2 m}\right) \frac{2 m}{\hbar^{2}}$, a Eq. (A.24) fica

$$
\begin{aligned}
\frac{1}{\tau_{s f}^{\uparrow \downarrow}}=\frac{m \Omega_{0}}{4 \hbar^{3}} & \left(N_{0} \alpha\right)^{2}\left\{S(S+1)-\left\langle S_{z}^{2}\right\rangle-\left\langle S_{z}\right\rangle\right\} \\
& \times \int_{L} x(z)\left|\chi^{\uparrow}\left(Z_{j}\right)\right|^{2}\left|\chi^{\downarrow}\left(Z_{j}\right)\right|^{2} \mathrm{~d} z .
\end{aligned}
$$




\section{A.4.2 Incluindo os fatores de Fermi}

Para incluir os fatores de Fermi eu considero a probabilidade de existir espaço de fase na sub-banda do estado final, ou seja, $1-f_{k_{\|}^{\bar{\sigma}}, m}$, com $f_{k_{\|}^{\bar{\sigma}}, m}$ a função de Fermi da sub-banda com elétrons minoritários. A taxa de espalhamento dada pela Eq. (A.5) de spin fica então

$$
\frac{1}{\tau_{s f}^{\sigma \bar{\sigma}}}=\frac{2 \pi}{\hbar} \sum_{k_{f}}\left\langle\left|\left\langle f\left|H_{s f}\right| i\right\rangle\right|^{2}\right\rangle_{s p i n}\left[1-f_{k_{\|}^{\bar{\sigma}}, m}\right] \delta\left(E_{f}^{\bar{\sigma}}-E_{i}^{\sigma}\right),
$$

com

$$
\left[1-f_{m}^{\bar{\sigma}}\left(\varepsilon_{k_{\|}}\right)\right]=\frac{1}{\exp \left[-\left(\varepsilon_{1}^{\sigma}+\varepsilon_{k_{\|}}-\mu_{m}^{\bar{\sigma}}\right) / k_{B} T_{e}\right]+1}
$$

$\mu_{M(m)}$ é o potencial químico de elétrons majoritários $(\mathrm{M})$ e minoritários $(\mathrm{m})$, dado por [39]

$$
\mu_{M(m)}^{\sigma}=\varepsilon_{1}^{e, \sigma}+k_{B} T \ln \left[\exp \left(\Delta_{M(m)}^{\sigma} / k_{B} T\right)-1\right],
$$

no qual $\Delta_{M(m)}$ é o excesso de energia correspondente à ocupação das sub-bandas majoritárias (M) e minoritárias $(\mathrm{m})$.

Substituindo (A.23) em (A.26) obtenho

$$
\begin{aligned}
& \frac{1}{\tau_{s f}^{\uparrow \downarrow}}=\frac{m \Omega_{0}}{4 \hbar^{3}}(\left.N_{0} \alpha\right)^{2}\{S(S+1) \\
& \times \int_{L} x(z)\left|\chi^{\uparrow}\left(Z_{j}\right)\right|^{2}\left|\chi^{\downarrow}\left(Z_{j}\right)\right|^{2} \mathrm{~d} z \\
& \times\left[1-f_{k_{\|}, m}\right] .
\end{aligned}
$$

De forma similar, para o caso de espalhamento da sub-banda majoritária com spin $|\downarrow\rangle$ para a sub-banda minoritária com spin $|\uparrow\rangle$, a taxa de espalhamento fica

$$
\begin{aligned}
& \frac{1}{\tau_{s f}^{\downarrow \uparrow}}=\frac{m \Omega_{0}}{4 \hbar^{3}}(\left.N_{0} \alpha\right)^{2}\{S(S+1) \\
& \times \int_{L} x(z)\left|\chi^{\uparrow}\left(Z_{j}\right)\right|^{2}\left|\chi^{\downarrow}\left(Z_{j}\right)\right|^{2} \mathrm{~d} z \\
& \times\left[1-f_{k_{\|}}, m\right]
\end{aligned}
$$

com a restrição que

$$
E_{1}^{\downarrow}+\frac{\hbar^{2} k_{\Perp}^{i^{2}}}{2 m} \geq E_{1}^{\uparrow}
$$




\section{Referências Bibliográficas}

[1] Michael von Ortenberg. Spin superlattice with tunable minigap. Phys. Rev. Lett., 49(14):1041-1043, 1982.

[2] N. Dai, H. Luo, F. C. Zhang, N. Samarth, M. Dobrowolska, e J. K. Furdyna. Spin superlattice formation in $\mathrm{ZnSe} / \mathrm{Zn}_{1-x} \mathrm{Mn}_{x}$ Se multilayers. Phys. Rev. Lett., 67(27):3824-3827, 1991.

[3] W. C. Chou, A. Petrou, J. Warnock, e B. T. Jonker. Spin superlattice behavior in $\mathrm{ZnSe} / \mathrm{Zn}_{0.99} \mathrm{Fe}_{0.01}$ Se quantum wells. Phys. Rev. Lett., 67(27): 3820-3823, 1991.

[4] S.A. Crooker, D. A. Tulchinsky, J. Levy, D. D. Awschalom, R. Garcia, e N. Samarth. Enhanced spin interactions in digital magnetic heterostructures. Phys. Rev. Lett., 75(3):505-508, 1995.

[5] J. Levy, V. Nikitin, J. M. Kikkawa, A. Cohen, N. Samarth, R. Garcia, e D. D. Awschalom. Spatiotemporal near-field spin microscopy in patterned magnetic heterostructures. Phys. Rev. Lett., 76(11):1948-1951, 1996.

[6] S. A. Crooker, J. J. Baumberg, F. Flack, N. Samarth, e D. D. Awschalom. Terahertz spin precession and coherent transfer of angular momenta in magnetic quantum wells. Phys. Rev. Lett., 77(13):2814-2817, 1996.

[7] S. A. Crooker, N. Samarth, e D. D. Awschalom. Quasi-two-dimensional spin distributions in II-VI magnetic semiconductor heterostructures: Clustering and dimensionality. Phys. Rev. B, 61(3):1736-1739, 2000.

[8] I. P. Smorchkova and N. Samarth. Fabrication of n-doped magnetic semiconductor heterostructures. Appl. Phys. Lett., 69(11):1640-1642, 1996.

[9] M. Julliere. Tunneling between ferromagnetic films. Phys. Lett. A, 54(3): 225-226, 1975.

[10] J. S. Moodera, Lisa R. Kinder, Terrilyn M. Wong, e R. Meservey. Large magnetoresistance at room temperature in ferromagnetic thin film tunnel junctions. Phys. Rev. Lett., 74(16):3273-3276, 1995. 
[11] M. N. Baibich, J. M. Broto, A. Fert, F. Nguyen Van Dau, F. Petroff, P. Eitenne, G. Creuzet, A. Friederich, e J. Chazelas. Giant magnetoresistance of (001)Fe/(001)Cr magnetic superlattices. Phys. Rev. Lett., 61(21):2472$2475,1988$.

[12] I. P. Smorchkova, N. Samarth, J. M. Kikkawa, e D. D. Awschalom. Spin transport and localization in a magnetic two-dimensional electron gas. Phys. Rev. Lett., 78(18):3571-3574, 1997.

[13] J. Carlos Egues. Spin-dependent perpendicular magnetotransport through a tunable $\mathrm{ZnSe} / \mathrm{Zn}_{1-x} \mathrm{Mn}_{x}$ Se heterostructure: A possible spin filter? Phys. Rev. Lett., 80(20):4578-4581, 1998.

[14] Gary A. Prinz. Spin-polarized transport. Physics Today, 48(4):58-63, 1995.

[15] S. A. Wolf, D. D. Awschalom, R. A. Buhrman, J. M. Daughton, S. Von Molnár, M. L. Roukes, A. Y. Chtchelkanova, e D. M. Treger. Spintronics: A spin-based electronics vision for the future. Science, 294(5546):14881494, 2001.

[16] John Schliemann, J. Carlos Egues, e Daniel Loss. Nonballistic spin-fieldeffect transistor. Phys. Rev. Lett., 90(14):146801, 2003.

[17] David D. Awschalom, Michael E. Flatté, e Nitin Samarth. Spintronics. Sci. Am., 286(6):66-73, 2002.

[18] Igor Zutic, Jaroslav Fabian, e S. Das Sarma. Spintronics: Fundamentals and applications. Rev. Mod. Phys., 76(2):323-410, 2004.

[19] S. Datta and B. Das. Electronic analog of the electrooptic modulator. Appl. Phys. Lett., 56(7):665-667, 1990.

[20] S. M. Girvin. Spin and isospin: Exotic order in quantum Hall ferromagnets. Phys. Today, 53(6):39-45, 2000.

[21] J. Jaroszyński, T. Andrearczyk, G. Karczewski, J. Wróbel, T. Wojtowicz, E. Papis, E. Kamińska, A. Piotrowska, Dragana Popović, e T. Dietl. Ising quantum Hall ferromagnet in magnetically doped quantum wells. Phys. Rev. Lett., 89:266802, 2002.

[22] E. P. De Poortere, E. Tutuc, S. J. Papadakis, e M. Shayegan. Resistance spikes at transitions between quantum Hall ferromagnets. Science, 290 (5496):1546-1549, 2000. 
[23] J. H. Smet, R. A. Deutschmann, W. Wegscheider, G. Abstreiter, e K. von Klitzing. Ising ferromagnetism and domain morphology in the fractional quantum Hall regime. Phys. Rev. Lett., 86(11):2412-2415, 2001.

[24] S. Koch, R. J. Haug, K. von Klitzing, e M. Razeghi. Suppression of the Landau-level coincidence: A phase transition in tilted magnetic fields. Phys. Rev. B, 47(7):4048-4051, 1993.

[25] V. Piazza, V. Pellegrini, F. Beltram, W. Wegscheider, T. Jungwirth, e A. H. MacDonald. First-order phase transitions in a quantum Hall ferromagnet. Nature, 402(6762):638-641, 1999.

[26] J. Eom, H. Cho, W. Kang, K. L. Campman, A. C. Gossard, M. Bichler, e W. Wegscheider. Quantum Hall ferromagnetism in a two-dimensional electron system. Science, 289(5488):2320-2323, 2000.

[27] Koji Muraki, Tadashi Saku, e Yoshiro Hirayama. Charge excitations in easy-axis and easy-plane quantum Hall ferromagnets. Phys. Rev. Lett., 87: 196801, 2001.

[28] E. P. De Poortere, E. Tutuc, Y. P. Shkolnikov, K. Vakili, M. Shayegan, E. Palm, e T. Murphy. Quantum hall effect in AlAs 2D electron systems. Int. J. Mod. Phys. B, 16(20-22):2917-2922, 2002.

[29] Michelle Chen, Woowon Kang, e Werner Wegscheider. Metamorphosis of the quantum Hall ferromagnet at $\nu=2 / 5$. Phys. Rev. Lett., 91(11):116804, 2003.

[30] E. P. De Poortere, E. Tutuc, e M. Shayegan. Critical resistance in the AlAs quantum Hall ferromagnet. Phys. Rev. Lett., 91(21):216802, 2003.

[31] Koji Muraki, Tadashi Saku, e Yoshiro Hirayama. Charge excitation and transport in pseudospin quantum Hall ferromagnets. Physica E, 18(1-3): 107-108, 2003.

[32] G. M. Gusev, A. A. Quivy, T. E. Lamas, J. R. Leite, O. Estibals, e J. C. Portal. Quantum Hall ferromagnet in a parabolic well. Phys. Rev. B, 67 (15):155313, 2003.

[33] Yoshiro Hirayama, Koji Murakia, Katsushi Hashimotoa, Kei Takashinaa, e Tadashi Saku. Quantum Hall effects at Landau level crossings. Physica E, 20(1-2):133-142, 2003. 
[34] K. B. Cooper, J. P. Eisenstein, L. N. Pfeiffer, e K. W. West. Metastable resistance-anisotropy orientation of two-dimensional electrons in high Landau levels. Phys. Rev. Lett., 92(2):026806, 2004.

[35] W. Desrat, F. Giazotto, V. Pellegrini, F. Beltram, F. Capotondi, G. Biasiol, L. Sorba, e D. K. Maude. Magneto-transport in high g-factor, low-density two-dimensional electron systems confined in $\mathrm{In}_{0.75} \mathrm{Ga}_{0.25} \mathrm{As} / \mathrm{In}_{0.75} \mathrm{Al}_{0.25} \mathrm{As}$ quantum wells. cond-mat/0401325, Jan 2004.

[36] J. Jaroszynski, T. Andrearczyk, G. Karczewski, J. Wróbel, T. Wojtowicz, E. Papis, E. Kaminska, A. Piotrowska, Dragana Popovic, e T. Dietl. Quantum hall ferromagnetism in II-VI based alloys. Phys. Stat. Sol. (b), 241(3): 712-717, 2004.

[37] R. Knobel, N. Samarth, J. G. E. Harris, e D. D. Awschalom. Measurements of Landau-level crossings and extended states in magnetic two-dimensional electron gases. Phys. Rev. B, 65:235327, 2002.

[38] J. G. E. Harris, R. Knobel, K. D. Maranowski, A. C. Gossard, N. Samarth, e D. D. Awschalom. Magnetization measurements of magnetic twodimensional electron gases. Phys. Rev. Lett., 86(20):4644-4647, 2001.

[39] J. Carlos Egues and John W. Wilkins. Spin-dependent phenomena in digital-magnetic heterostructures: Clustering and phase-space filling effects. Phys. Rev. B, 58(24):R16012-R16015, 1998.

[40] P. Hohenberg and W. Kohn. Inhomogeneous electron gas. Phys. Rev., 136 (3B):B864-B871, 1964.

[41] W. Kohn and L. J. Sham. Self-consistent equations including exchange and correlation effects. Phys. Rev., 140(4A):A1133, 1965.

[42] J. M. Luttinger and W. Kohn. Motion of electrons and holes in perturbed periodic fields. Phys. Rev., 97(4):869-883, 1955.

[43] G. Bastard. Hydrogenic impurity states in a quantum well: A simple model. Phys. Rev. B, 24(8):4714-4722, 1981.

[44] G. Bastard. Theoretical investigations of superlattice band structure in the envelope-function approximation. Phys. Rev. B, 25(12):7584-7597, 1982.

[45] J. A. Gaj, R. Planel, e F. Fishman. Relation of magneto-optical properties of free excitons to spin alignment of $\mathrm{Mn}^{2+}$ ions in $\mathrm{Cd}_{1-x} \mathrm{Mn}_{x}$ Te. Solid State Commun., 29(5):435-438, 1979. 
[46] Tomasz Wojtowicz, Grzegorz Karczewski, e Jacek Kossut. Excitons in novel diluted magnetic semiconductor quantum structures. Thin Solid Films, 306 (2):271-282, 1997.

[47] A. C. Gossard, P. M. Petroff, W. Weigmann, R. Dingle, e A. Savage. Epitaxial structures with alternate-atomic-layer composition modulation. Appl. Phys. Lett., 29(6):323-325, 1976.

[48] J. K. Furdyna. Diluted magnetic semiconductors. J. Appl. Phys., 64(4): R29-R64, 1988.

[49] T. Lebihen, E. Deleporte, e C. Delalande. Band-offset determination of the CdTe/(Cd,Mn)Te interface. Phys. Rev. B, 55(3):1724-1729, 1997.

[50] F. Liaci, P. Bigenwald, O. Briot, B. Gil, N. Briot, T. Cloitre, e R. L. Aulombard. Band offsets and exciton binding energies in $\mathrm{Zn}_{1-x} \mathrm{Cd}_{x} \mathrm{Se}-\mathrm{ZnSe}$ quantum wells grown by metal-organic vapor-phase epitaxy. Phys. Rev. B, 51(7):4699-4702, 1995.

[51] J. A. Gaj. Magnetooptical properties of large-gap diluted magnetic semiconductors. In J. K. Furdyna and J. Kossut, editores, Semiconductors and Semimetals / Diluted Magnetic Semiconductors, volume 25 de Semiconductors and Semimetals, capítulo 7, páginas 275-309. Academic Press, San Diego, 1988.

[52] W. M. Becker. Band structure and optical properties of wide-gap $\mathrm{A}_{1-x}^{I I} \mathrm{Mn}_{x} \mathrm{~B}^{V I}$ alloys at zero magnetic field. In Jacek K. Furdyna and Jacek Kossut, editores, Diluted Magnetic Semiconductors, capítulo 2, páginas 35-72. Academic Press, San Diego, 1988.

[53] P. J. Klar, D. Wolverson, J. J. Davies, W. Heimbrodt, e M. Happ. Determination of the chemical valence-band offset for $\mathrm{Zn}_{1-x} \mathrm{Mn}_{x} \mathrm{Se} / \mathrm{ZnSe}$ multiplequantum-well structures of high $x$. Phys. Rev. B, 57(12):7103-7112, 1998.

[54] Jacek Kossut. Band structure and quantum transport phenomena in narrow-gap diluted magnetic semiconductors. In J. K. Furdyna and J. Kossut, editores, Semiconductors and Semimetals / Diluted Magnetic Semiconductors, volume 25, capítulo 5, páginas 183-227. Academic Press, San Diego, 1988.

[55] G. Bastard, C. Rigaux, Y. Guldner, J. Mycielski, e A. Mycielski. Effect of exchange on the impurity magneto-absorption in zero-gap $\mathrm{Hg}_{1-k} \mathrm{Mn}_{k} \mathrm{Te}$ mixed-crystals. J. Phys. (Paris), 39(1):87-98, 1978. 
[56] P. J. Cooney, E. P. Kanter, e Z. Vager. Convenient numerical technique for solving the one-dimensional schrödinger equation for bound states. Am. J. Phys., 49(1):76-77, 1981.

[57] William H. Press, Saul A. Teukolsky, William T. Vetterling, e Brian P. Flannery. Numerical Recipes in $C$ : The Art of Scientific Computing. Cambridge University Press, NY, $2^{a}$ edição, 1992.

[58] D. U. von Rosenberg. Methods for the Numerical Solution of Partial Differential Equations. Elsevier, New York, 1969.

[59] J. A. Gaj, W. Grieshaber, C. Bodin-Deshayes, J. Cibert, G. Feuillet, Y. Merle D'Aubigné, e A. Wasiela. Magneto-optical study of interface mixing in the CdTe-(Cd,Mn)Te system. Phys. Rev. B, 50(8):5512-5527, 1994.

[60] D. D. Awschalom and Nitin Samarth. Spin dynamics and quantum transport in magnetic semiconductor quantum structures. J. Magn. Magn. Mater., 200(1-3):130-147, 1999.

[61] W. Grieshaber, A. Haury, J. Cibert, Y. Merle d'Aubigné, A. Wasiela, e J. A. Gaj. Magneto-optic study of the interface in semimagnetic semiconductor heterostructures: Intrinsic effect and interface profile in $\mathrm{CdTe}-\mathrm{Cd}{ }_{1-x} \mathrm{Mn}_{x} \mathrm{Te}$. Phys. Rev. B, 53(8):4891-4904, 1996.

[62] W. Grieshaber, J. Cibert, J. A. Gaj, Y. Merle d' Aubigné, e A. Wasiela. Profiles of the normal and inverted semiconductor interfaces: A Zeeman study in asymmetric $\mathrm{Cd}_{1-y} \mathrm{Zn}_{y} \mathrm{Te} / \mathrm{CdTe} / \mathrm{Cd}_{1-x} \mathrm{Mn}_{x}$ Te quantum wells. Phys. Rev. B, 50(3):2011-2014, 1994.

[63] G. Schmitt, B. Kuhn-Heinrich, U. Zehnder, W. Ossau, T. Litz, A. Waag, e G. Landwehr. Characterization of interfaces in semimagnetic quantum wells. Acta Phys. Pol. A, 88(5):897-900, 1995.

[64] A. Lemaître, C. Testelin, C. Rigaux, S. Mackowski, Nguyen The Khoi, J. A. Gaj, G. Karczewski, T. Wojtowicz, e J. Kossut. Characterization of normal and inverted interfaces by the zeeman effect in $\mathrm{Cd}_{1-x} \mathrm{Mn}_{x} \mathrm{Te} / \mathrm{CdTe} / \mathrm{Cd}_{1-y} \mathrm{Mg}_{y} \mathrm{Te}$ quantum wells. Phys. Rev. B, 57(8): 4708-4712, 1998.

[65] A. Kudelski, A. Golnik, J. A. Gaj, F. V. Kyrychenko, G. Karczewski, T. Wojtowicz, Yu. G. Semenov, O. Krebs, e P. Voisin. Interface profiles and inplane anisotropy in common anion type-I $\mathrm{Cd}_{1-x} \mathrm{Mg}_{x} \mathrm{Te} / \mathrm{CdTe} / \mathrm{Cd}_{1-x} \mathrm{Mn}_{x} \mathrm{Te}$ heterostructures studied by reflectivity. Phys. Rev. B, 64(4):045312, 2001. 
[66] M. Syed, G. L. Yang, J. K. Furdyna, M. Dobrowolska, S. Lee, e L. R. Ram-Mohan. Role of magnetic/nonmagnetic semiconductor interfaces in magneto-optical properties of small-offset superlattices. Phys. Rev. B, 66 (7):075213, 2002.

[67] J. M. Fatah, T. Piorek, P. Harrison, T. Stirner, e W. E. Hagston. Numerical simulation of antiferromagnetic spin-pairing effects in diluted magnetic semiconductors and enhanced paramagnetism at interfaces. Phys. Rev. B, 49(15):10341-10344, 1994.

[68] Y. Shapira and V. Bindilatti. Magnetization-step studies of antiferromagnetic clusters and single ions: Exchange, anisotropy, e statistics. J. Appl. Phys., 92(8):4155-4185, 2002.

[69] Robert E. Behringer. Number of single, double, e triple clusters in a system containing two types of atoms. J. Chem Phys., 29(3):537-539, 1958.

[70] Marshall M. Kreitman, Frederick J. Milford, Richard P. Kenan, e J. G. Daunt. Magnetic susceptibility of $\mathrm{Mn}^{2+}$ in CdS and effects of antiferromagnetic exchange. Phys. Rev., 144(2):367-372, 1966.

[71] Y. Shapira, S. Foner, D. H. Ridgley, K. Dwight, e A. Wold. Technical saturation and magnetization steps in diluted magnetic-semiconductors: Predictions and observations. Phys. Rev. B, 30(7):4021-4023, 1984.

[72] W. Ossau, B. Kuhn-Heinrich, A. Waag, e G. Landwehr. Enhanced paramagnetism in ultrathin $\mathrm{Cd}_{1-x} \mathrm{Mn}_{x}$ Te layers. J. Cryst. Growth, 1046-1051: 1046-1051, 1996.

[73] O. Dehaese, X. Wallart, e F. Mollot. Kinetic model of element III segregation during molecular beam epitaxy of III-III'-V semiconductor compounds. Appl. Phys. Lett., 66(1):52-54, 1995.

[74] J. M. Moison, C. Guille, F. Houzay, F. Barthe, e M. Van Rompay. Surface segregation of third-column atoms in group III-V arsenide compounds: Ternary alloys and heterostructures. Phys. Rev. B, 40(9):6149-6162, 1989.

[75] G. S. Spencer, J. Menéndez, L. N. Pfeiffer, e K. W. West. Optical-phonon raman-scattering study of short-period GaAs-AlAs superlattices: An examination of interface disorder. Phys. Rev. B, 52(11):8205-8218, 1995.

[76] Belita Koiller, R. B. Capaz, e H. Chacham. Segregation, interface morphology, and the optical properties of GaAs/AlAs quantum wells: A theoretical study. Phys. Rev. B, 60(3):1787-1791, 1999. 
[77] Paul G. Shewmon. Diffusion in Solids. McGraw-Hil Series in Materials Science and Engineering. McGraw-Hill, New York, 1963.

[78] P. Harrison, J. M. Fatah, T. Stimer, e W. E. Hagston. Alloy nonrandomness in diluted magnetic semiconductors. J. Appl. Phys., 79(3):1684-1688, 1996.

[79] C. Rigaux. Magnetooptics in narrow-gap diluted magnetic semiconductors. In J. K. Furdyna and J. Kossut, editores, Semiconductors and Semimetals / Diluted Magnetic Semiconductors, volume 25 de Semiconductors and Semimetals, capítulo 6, páginas 229-274. Academic Press, San Diego, 1988.

[80] S. A. Crooker, D. G. Rickel, I. P. Smorchkova, N. Samarth, J. M. Kikkawa, e D. D. Awschalom. Optical signatures from magnetic two-dimensional electron gases in magnetic fields to 60 T. J. Appl. Phys., 85(8):5932-5934, 1999.

[81] J. Kossut. Scattering of conduction electrons by magnetic-impurities in semiconductors of InSb-type and HgTe-type band-structure. Phys. Status Solidi B, 72(1):359-367, 1975.

[82] G. Bastard and L. L. Chang. Spin-flip relaxation time of conduction electrons in $\mathrm{Cd}_{1-x} \mathrm{Mn}_{x}$ Te quantum wells. Phys. Rev. B, 41(11):7899-7902, 1990.

[83] J. Carlos Egues. Spin-Dependent Phenomena in Mn-Based Semiconductor Heterostructures. Tese de doutorado, The Ohio State University, 1996.

[84] John H. Davies, D. M. Bruls, J. W. A. M. Vugs, e P. M. Koenraad. Relaxation of a strained quantum well at a cleaved surface. J. Appl. Phys., 91 (7):4171-4176, 2002.

[85] D. M. Bruls, J. W. A. M. Vugs, P. M. Koenraad, H. W. M. Salemink, J. H. Wolter, M. S. Skolnick, Fei Long, e S. P. A. Gill. Determination of the shape and indium distribution of low-growth-rate InAs quantum dots by cross-sectional scanning tunneling microscopy. Appl. Phys. Lett., 81(9): 1708-1710, 2002.

[86] P.M. Koenraad, D.M. Brulsa, J.H. Davies, S.P.A. Gillc, Fei Long, M. Hopkinsond, M. Skolnickd, e J.H. Woltera. Composition profiling at the atomic scale in III-V nanostructures by cross-sectional STM. Physica E, 17:526$532,2003$.

[87] P. Offermans, P. M. Koenraad, J. H. Wolter, J. D. Song, Jong Min Kim, Seong Ju Bae, e Yong Tak Lee. Annealing of InGaAlAs digital alloy stu- 
died with scanning-tunneling microscopy and filled-states topography. Appl. Phys. Lett., 82(8):1191-1193, 2003.

[88] P. Offermans, P. M. Koenraad, J. H. Wolter, M. Beck, T. Aellen, e J. Faist. Digital alloy interface grading of an InAlAs/InGaAs quantum cascade laser structure studied by cross-sectional scanning tunneling microscopy. Appl. Phys. Lett., 83(20):4131-4133, 2003.

[89] S. M. Girvin. The quantum Hall effect: Novel excitations and broken symmetries. cond-mat/9907002, 1999.

[90] K. von Klitzing, G. Dorda, e M. Pepper. New method for high-accuracy determination of the fine-structure constant based on quantized Hall resistance. Phys. Rev. Lett., 45(6):494-497, 1980.

[91] D. C. Tsui, H. L. Stormer, e A. C. Gossard. Two-dimensional magnetotransport in the extreme quantum limit. Phys. Rev. Lett., 48(22):1559-1562, 1982.

[92] R. B. Laughlin. Anomalous quantum Hall effect: An incompressible quantum fluid with fractionally charged excitations. Phys. Rev. Lett., 50(18): 1395-1398, 1983.

[93] R. Dingle, H. L. Störmer, A. C. Gossard, e W. Wiegmann. Electron mobilities in modulation-doped semiconductor heterojunction superlattices. Appl. Phys. Lett., 33(7):665-667, 1978.

[94] W. Kohn. Nobel lecture: Electronic structure of matter-wave functions and density functionals. Rev. Mod. Phys., 71(5):1253-1266, 1999.

[95] H. H. March. Origins - The Thomas-Fermi theory. In S. Lundqvist and N. H. March, editores, Theory of the Inhomogeneous Electron Gas, capítulo 1, páginas 1-77. Plenum, New York, 1983.

[96] D. M. Ceperley and B. J. Alder. Ground state of the electron gas by a stochastic method. Phys. Rev. Lett., 45(7):566-569, 1980.

[97] C. Filippi, C. J. Umrigar, e M. Taut. Comparison of exact and approximate density functionals for an exactly soluble model. J. Chem. Phys., 100(2): 1290-1296, 1994.

[98] W. Kohn and P. Vashishta. General density funtional theory. In S. Lundqvist and N. H. March, editores, Theory of the Inhomogeneous Electron Gas, capítulo 2, páginas 79-147. Plenum, New York, 1983. 
[99] S. H. Vosko, L. Wilk, e M. Nusair. Accurate spin-dependent electron liquid correlation energies for local spin-density calculations - a critical analysis. Can. J. Phys., 58(8):1200-1211, 1980.

[100] U. von Barth and L. Hedin. Local exchange-correlation potential for spin polarized case .1. J. Phys. C, 5(13):1629-1642, 1972.

[101] O. Gunnarsson and B. I. Lundqvist. Exchange and correlation in atoms, molecules, e solids by the spin-density-functional formalism. Phys. Rev. B, 13(10):4274-4298, 1976.

[102] J. P. Perdew and Alex Zunger. Self-interaction correction to densityfunctional approximations for many-electron systems. Phys. Rev. B, 23 (10):5048-5079, 1981.

[103] John P. Perdew and Yue Wang. Accurate and simple analytic representation of the electron-gas correlation energy. Phys. Rev. B, 45(23):13244-13249, 1992.

[104] John P. Perdew and Yue Wang. Accurate and simple density functional for the electronic exchange energy: Generalized gradient approximation. Phys. Rev. B, 33(12):8800-8802, 1986.

[105] John P. Perdew. Density-functional approximation for the correlation energy of the inhomogeneous electron gas. Phys. Rev. B, 33(12):8822-8824, 1986.

[106] A. D. Becke. Density-functional exchange-energy approximation with correct asymptotic behavior. Phys. Rev. A, 38(6):3098-3100, 1988.

[107] John P. Perdew. Unified theory of exchange and correlation beyond the local density approximation. In P. Ziesche and H. Eschrig, editores, Electronic Structure of Solids '91, páginas 11-20. Akademie Verlag, Berlim, 1991.

[108] K. Burke, John P. Perdew, e Y. Wang. Derivation of a generalized gradient approximation: The PW91 density functional. In J. F. Dobson, G. Vignale, e M. P. Das, editores, Electronic Density Functional Theory: Recent Progress and New Directions, page 81. Plenum, New York, 1997.

[109] John P. Perdew, Kieron Burke, e Matthias Ernzerhof. Generalized gradient approximation made simple. Phys. Rev. Lett., 77(18):3865-3868, 1996.

[110] R. van Leeuwen and J. Baerends. Exchange-correlation potential with correct asymptotic behavior. Phys. Rev. A, 49(4):2421-2431, 1994. 
[111] John P. Perdew and Stefan Kurth. Density functionals for non-relativistic coulomb systems. In Daniel Joubert, editor, Density Functionals: Theory and Applications, páginas 8-59. Springer, Berlin, 1998.

[112] Yue Wang and John P. Perdew. Spin scaling of the electron-gas correlation energy in the high-density limit. Phys. Rev. B, 43(11):8911-8916, 1991.

[113] N. David Mermin. Thermal properties of the inhomogeneous electron gas. Phys. Rev., 137(5A):A1441, 1965.

[114] Neil W. Ashcroft and N. David Mermin. Solid State Physics. Saunders College, Orlando, 1976.

[115] John P. Perdew and Mel Levy. Extrema of the density functional for the energy: Excited states from the ground-state theory. Phys. Rev. B, 31(10): 6264-6272, 1985.

[116] Hideo Aoki. Quantized Hall effect. Rep. Prog. Phys., 50(6):655-730, 1987.

[117] H. Aoki and T. Ando. Effect of localization on the Hall conductivity in the two-dimensional system in strong magnetic-fields. Solid State Commun., 38(11):1079-1082, 1981.

[118] T. Ando and Y. Uemura. Theory of quantum transport in a 2-dimensional electron-system under magnetic-fields . I. characteristics of level broadening and transport under strong fields. J. Phys. Soc. Jpn., 36(4):959-967, 1974.

[119] Rolf R. Gerhardts. Path-integral approach to 2-dimensional magnetoconductivity problem. 1. General formulation of approach. Z. Phys. B, 21(3):275-283, 1975.

[120] Rolf. R. Gerhardts. Path-integral approach to 2-dimensional magnetoconductivity problem. 2. Application to n-type (100)-surface inversion layers of p-silicon. Z. Phys. B, 21(3):285-294, 1975.

[121] Rolf R. Gerhardts. Cumulant approach to the two-dimensional magnetoconductivity problem. Surf. Sci., 58(1):227-234, 1976.

[122] M. van der Burgt, V. C. Karavolas, F. M. Peeters, J. Singleton, R. J. Nicholas, F. Herlach, J. J. Harris, M. van Hove, e G. Borghs. Magnetotransport in a pseudomorphic GaAs/Ga0.8In0.2As/Ga0.75A10.25As heterostructure with a Si delta-doping layer. Phys. Rev. B, 52(16):12218-12231, 1995. 
[123] Tsuneya Ando. Theory of quantum transport in a two-dimensional electron system under magnetic fields. III. Many-site approximation. J. Phys. Soc. Jpn., 37(3):622-630, 1974.

[124] Tsuneya Ando. Theory of cyclotron resonance lineshape in a twodimensional electron system. J. Phys. Soc. Jpn., 38(4):989-997, 1975.

[125] A. Potts, R. Shepherd, W. G. Herrenden-Harker, M. Elliott, C. L. Jones, A. Usher, G. A. C. Jones, D. A. Ritchie, E. H. Linfield, e M. Grimshaw. Magnetization studies of landau level broadening in two-dimensional electron systems. J. Phys.: Condens. Matter, 8(7):5189-5207, 1996.

[126] D. Shoenberg. Magnetization of a two-dimensional electron gas. J. Low Temp. Phys., 56(5-6):417-440, 1984.

[127] I. D. Vagner. Electrons in magnetic fields. Technical report, Universite Josef Fourier - Max Planck Institute, http://www.magniel.com/hmtj/education/lectures/vag1.html, 1999.

[128] J. P. Eisenstein, H. L. Stormer, V. Narayanamurti, A. Y. Cho, A. C. Gossard, e C. W. Tu. Density of states and de Haas-van Alphen effect in two-dimensional electron systems. Phys. Rev. Lett., 55(8):875-878, 1985.

[129] Kerson Huang. Statistical Mechanics. Wiley, New York, $2^{a}$ edição, 1987.

[130] Tsuneya Ando. Theory of quantum transport in a two-dimensional electron system under magnetic fields. II. Single-site approximation under strong fields. J. Phys. Soc. Jpn., 36(6):1521-1529, 1974.

[131] Tsuneya Ando. Theory of quantum transport in a two-dimensional electron system under magnetic fileds. IV. Oscillatory conductivity. J. Phys. Soc. Jpn., 37(5):1233-1237, 1974.

[132] T. Ando, Y. Matsumoto, e Y. Uemura. Theory of Hall-effect in a 2dimensional electron-system. J. Phys. Soc. Jpn., 39(2):279-288, 1975.

[133] Edwin H. Hall. On a new action of the magnet on electric currents. Am. J. Math., 2:287, 1879.

[134] Supriyo Datta. Electronic transport in mesoscopic systems. In Haroon Ahmed, Michael Pepper, e Alec Broers, editores, Cambridge Studies in Semiconductor Physics and Microelectronic Engineering. Cambridge University Press, Cambridge, New York, 1995. 
[135] Tapash Chakraborty and Pekka Pietiläinen. The Quantum Hall Effects. Integral and Fractional, volume 85 de Springer Series in Solid-State Sciences. Springer, Berlin, $2^{a}$ edição, 1995.

[136] R. E. Prange. Quantized Hall resistance and the measurement of the finestructure constant. Phys. Rev. B, 23(9):4802-4805, 1981.

[137] R. Knobel, N. Samarth, S. A. Crooker, e D. D. Awschalom. Spin-polarized quantum transport and magnetic field-dependent carrier density in magnetic two-dimensional electron gases. Physica E, 6(1-4):786-789, 2000.

[138] Henrique J. P. Freire and J. Carlos Egues. Subband structure of II-VI modulation-doped magnetic quantum wells. Braz. J. Phys., 32(2A):327$330,2002$.

[139] Henrique J. P. Freire and J. Carlos Egues. "Reservoir model" for shallow modulation-doped digital magnetic quantum wells. J. Supercond., 16(2): 299-302, 2003.

[140] J. G. E. Harris, R. Knobel, K. D. Maranowski, A. C. Gossard, N. Samarth, e D. D. Awschalom. Damping of micromechanical structures by paramagnetic relaxation. Appl. Phys. Lett., 82(20):3532-3534, 2003.

[141] J. G. E. Harris, D. D. Awschalom, F. Matsukura, H. Ohno, K. D. Maranowski, e A. C. Gossard. Integrated micromechanical cantilever magnetometry of Ga1-xMnxAs. Appl. Phys. Lett., 75(8):1140-1142, 1999.

[142] J. G. E. Harris, D. D. Awschalom, K. D. Maranowski, e A. C. Gossard. Magnetization and dissipation measurements in the quantum hall regime using an integrated micromechanical magnetometer. J. Appl. Phys., 87(9): 5102-5104, 2000.

[143] S. A. J. Wiegers, M. Specht, L. P. Lévy, M. Y. Simmons, D. A. Ritchie, A. Cavanna, B. Etienne, G. Martinez, e P. Wyder. Magnetization and energy gaps of a high-mobility $2 \mathrm{~d}$ electron gas in the quantum limit. Phys. Rev. Lett., 79(17):3238-3241, 1997.

[144] I. Meinel, T. Hengstmann, D. Grundler, D. Heitmann, W. Wegscheider, e M. Bichler. Magnetization of the fractional quantum Hall states. Phys. Rev. Lett., 82(4):819-822, 1999.

[145] Robert G. Knobel. Two-Dimensional Electron Gases in Diluted Magnetic Semiconductors. Tese de doutorado, Pennsylvania State University, 2000. 
[146] H. K. Ng, Y. A. Leem, R. Knobel, I. P. Smorchkova, A. A. Sirenko, e N. Samarth. Cyclotron resonance in modulation-doped $\mathrm{ZnSe} / \mathrm{Zn}_{1-x} \mathrm{Cd}_{x} \mathrm{Se}$ and ZnTe/CdSe single quantum wells. Appl. Phys. Lett., 75(23):3662-3664, 1999.

[147] H. Leiderer, W. Limmer, e W. Gebhardt. Resonant raman-scattering by optical phonons in $\mathrm{ZnSe}$ and $\mathrm{Zn}_{0.97} \mathrm{Mn}_{0.03}$ Se near the $\mathrm{E} 0$ gap. J. Cryst. Growth, 101(1-4):921-925, 1990.

[148] O. Madelung, M. Schulz, e H. Weiss. Semiconductors. In K. H. Hellwege and O. Madelung, editores, Landolt-Börnstein: Numerical Data and Functional Relationships in Science and Technology, volume 17 de New Series, Group III: Condensed Matter. Springer-Verlag, Berlin, 1984.

[149] G. V. Astakhov, D. R. Yakovlev, V. P. Kochereshko, W. Ossau, W. Faschinger, J. Puls, F. Henneberger, S. A. Crooker, Q. McCulloch, D. Wolverson, N. A. Gippius, e A. Waag. Binding energy of charged excitons in ZnSe-based quantum wells. Phys. Rev. B, 65:165335, 2002.

[150] A. Twardowski, M. Von Ortenberg, M. Demianiuk, e R. Pauthenet. Magnetization of ZnMnTe, ZnMnSe and ZnMnS mixed-crystals. Acta. Phys. Pol. A, 67(2):339-343, 1985.

[151] D. Theis. Wavelength-modulated reflectivity spectra of ZnSe and ZnS from 2.5 to 8-eV. Phys. Status Solidi B, 79(1):125-130, 1977.

[152] A. Twardowski, T. Dietl, e M. Demianiuk. The study of the s-d type exchange interaction in $\mathrm{Zn}_{1-x} \mathrm{Mn}_{x}$ Se mixed crystals. Solid State Commun., 48(10):845-848, 1983.

[153] G. F. Giuliani and J. J. Quinn. Spin-polarization instability in a tilted magnetic field of a two-dimensional electron gas with filled Landau levels. Phys. Rev. B, 31(10):6228-6232, 1985.

[154] T. Jungwirth and A. H. MacDonald. Resistance spikes and domain wall loops in ising quantum Hall ferromagnets. Phys. Rev. Lett., 87:216801, 2001.

[155] T. Jungwirth and A. H. MacDonald. Pseudospin anisotropy classification of quantum Hall ferromagnets. Phys. Rev. B, 63:035305, 2000.

[156] John Schliemann, J. Carlos Egues, e Daniel Loss. Variational study of the $\mathrm{nu}=1$ quantum Hall ferromagnet in the presence of spin-orbit interaction. Phys. Rev. B, 67(8):085302, 2003. 
[157] E. H. Rezayi, T. Jungwirth, A. H. MacDonald, e F. D. M. Haldane. Exact diagonalization study of domain structure in integer filling factor quantum Hall ferromagnets. Phys. Rev. B, 67(20):201305, 2003.

[158] Gérald Bastard. Wave Mechanics Applied to Semiconductor Heterostructures. Monographies de Physique. Les Editions de Physique, Les Ulis Cedex, 1988.

[159] S. L. Sondhi, S. M. Girvin, J. P. Carini, e D. Shahar. Continuous quantum phase transitions. Rev. Mod. Phys., 69(1):315-333, 1997.

[160] Subir Sachdev. Quantum phase transitions. Physics World, 12(4):33-38, April 1999.

[161] Matthias Vojta. Quantum phase transitions. Rep. Prog. Phys., 66(12): 2069-2110, 2003.

[162] G. Karczewski and Yong-Jie. Wang. Resonant coupling of cyclotron and spin resonances in modulation-doped $\mathrm{CdMnTe} / \mathrm{CdMgTe}$ quantum well structure. Physica B, 298:402, 2001.

[163] M. M. Fogler and B. I. Shklovskii. Collapse of spin splitting in the quantum Hall effect. Phys. Rev. B, 52(24):17366-17378, 1995.

[164] T. Jungwirth, A. H. MacDonald, e E. H. Rezayi. Two-dimensional Ising physics in quantum Hall ferromagnets. Physica E, 12:1-7, 2002.

[165] D. Tonnies, G. Bacher, A. Forcher, A. Waag, T. Litz, e G. Landwehr. Optical Study Of Intermixing In CdTe/CdMgTe Quantum-Wells. Japan J. Appl. Phys., 33:L247-L249, 1994.

[166] H. L. Stormer and D. C. Tsui. The quantized Hall-effect. Science, 220 (4603):1241-1246, 1983.

[167] Klaus von Klitzing. The quantized Hall effect. Rev. Mod. Phys., 58(3): 519-531, 1986.

[168] B. I. Halperin. The quantized Hall-effect. Sci. Am., 52(4):40-48, 1986.

[169] J. K. Jain. Composite fermions. In Sankar Das Sarma and Aron Pinczuk, editores, Perspectives in Quantum Hall Effects, capítulo 7, páginas 265-305. Wiley, New York, 1997.

[170] H. L. Stormer and D. C. Tsui. Composite fermions in the fractional quantum Hall effect. In Sankar Das Sarma and Aron Pinczuk, editores, Perspectives 
in Quantum Hall Effects, capítulo 10, páginas 385-421. Wiley, New York, 1997. 